\title{
Cooperative Molybdenum-Thiolate Reactivity for Transfer Hydrogenation of Nitriles
}

Shu-Fen Hou, ${ }^{\dagger}$ Jia-Yi Chen,,$^{\dagger \dagger}$ Minghui Xue, ${ }^{\dagger}$ Mengjing Jia, ${ }^{\dagger}$ Xiaofang Zhai, ${ }^{\dagger}$ RongZhen Liao, ${ }^{* \dagger 广}$ Chen-Ho Tung, ${ }^{\dagger}$ and Wenguang Wang*

$\dagger$ Key Lab of Colloid and Interface Chemistry, Ministry of Education, School of Chemistry and Chemical Engineering, Shandong University, No. 27 South Shanda Road, Jinan, 250100, China

${ }^{\dagger}$ School of Chemistry and Chemical Engineering, Huazhong University of Science and Technology, 1037 Luoyu Road, Wuhan, 430074, China

*E-mail: wwg@sdu.edu.cn; rongzhen@hust.edu.cn 


\section{Table of content}

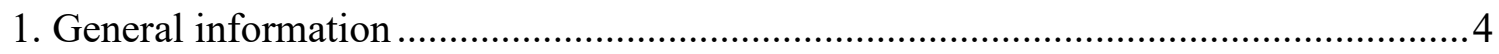

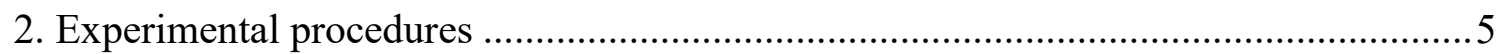

2.1 Synthesis of molybdenum complexes.........................................................

2.2 Screening of hydrogen sources of nitriles hydrogenation.................................

2.3 General procedures for the hydrogenation of nitriles ..................................... 8

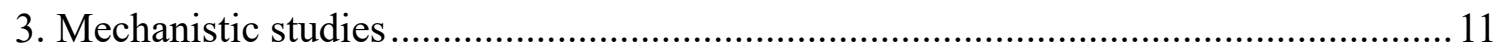

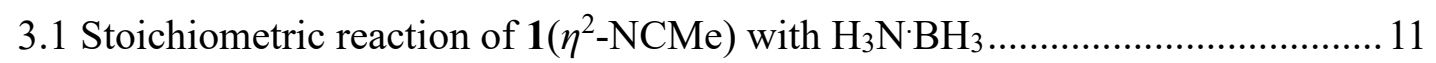

3.2 Stoichiometric reaction of $1 \mathrm{H}\left(\mathrm{BH}_{2}\right)$ with 4-Methoxybenzonitrile ..................... 13

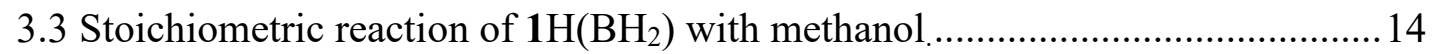

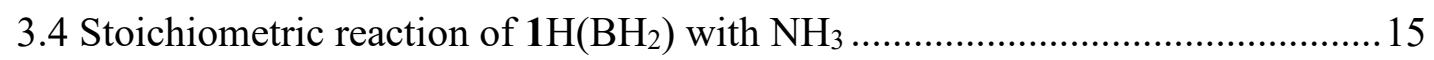

3.5 Stoichiometric reaction of $1 \mathrm{H}\left(\mathrm{BH}_{2}\right)$ with $\mathrm{NH}_{3}$ and 4-methoxybenzonitrile. ......20

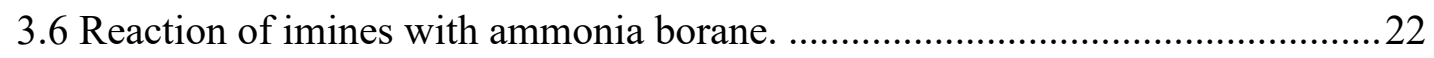

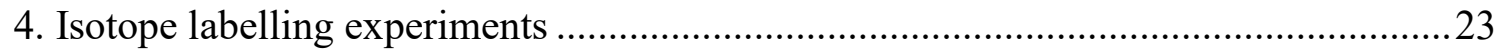

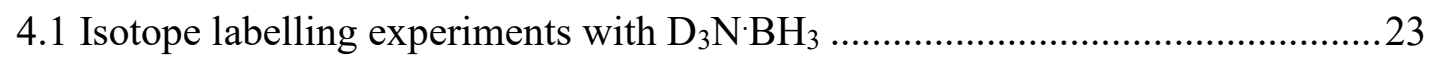

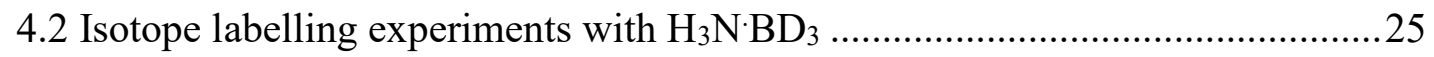

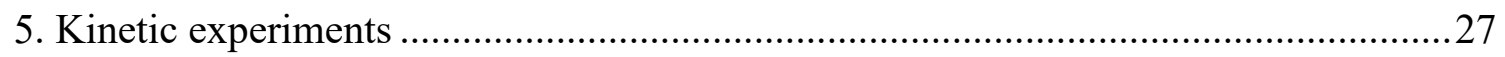

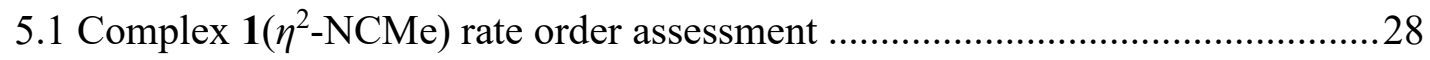

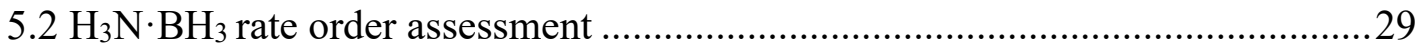

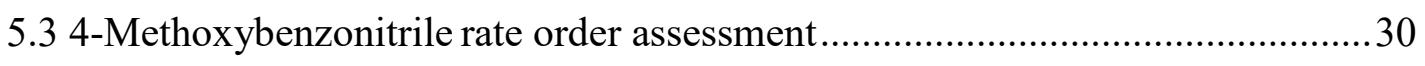

5.4 Determination of the kinetic isotope effect ........................................................ 31

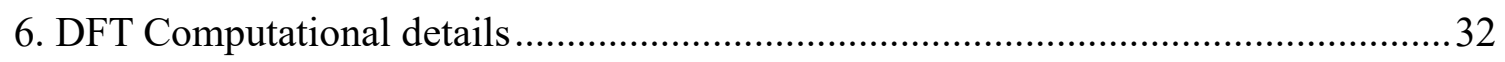

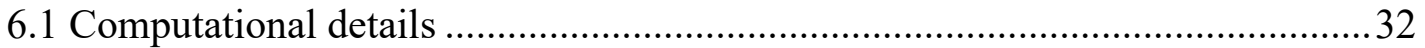

6.2 Reaction between catalyst $\mathbf{1}\left(\eta^{2}-\mathrm{NCMe}\right), \mathrm{CH}_{3} \mathrm{CN}$, and $\mathrm{H}_{3} \mathrm{~N} \cdot \mathrm{BH}_{3} \ldots \ldots \ldots \ldots \ldots \ldots . . . . . . . . . .32$

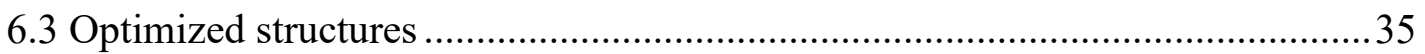

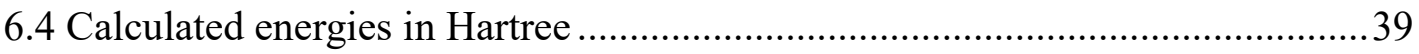

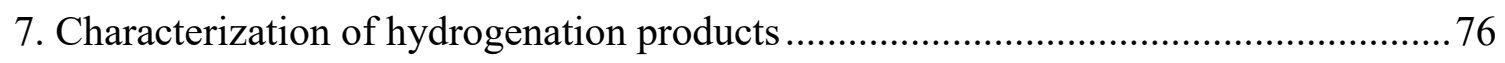

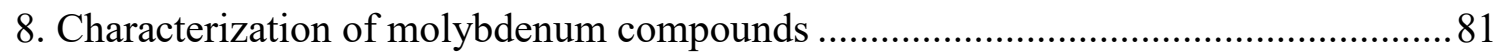

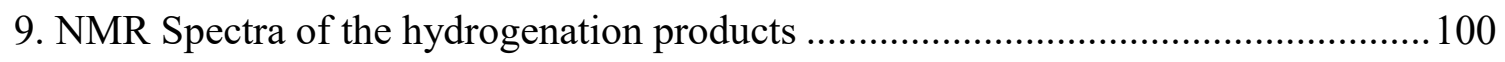




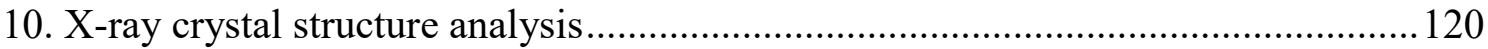

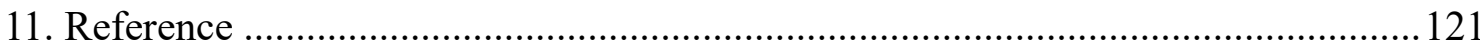




\section{General information}

All reactions were performed in flame-dried glassware using standard Schlenk techniques or in a glovebox under nitrogen atmosphere. Toluene, tetrahydrofuran, dichloromethane, ether, hexane and acetonitrile were dried and degassed by Solvent Purification Systems (Innovative Technology). $\mathrm{C}_{6} \mathrm{D}_{6}, \mathrm{CDCl}_{3}, \mathrm{CD}_{2} \mathrm{Cl}_{2}, \mathrm{CD}_{3} \mathrm{CN}$ were dried with $4 \AA$ molecular sieves and degassed by freeze-pump-thaw methods. Ferrocenium tetrafluoroborate, bis(cyclopentadienyl)cobalt, nitriles, $\mathrm{D}_{2} \mathrm{O}$ and tetraethylsilane were purchased from commercial suppliers. The 2-(diphenylphosphino)benzenethiol $\left(\mathrm{Ph}_{2} \mathrm{PC}_{6} \mathrm{H}_{4} \mathrm{SH}\right)^{1}$ and $\left(\mathrm{Cp} * \mathrm{Mo}(\mathrm{CO})_{3} \mathrm{Cl}\right)^{2}$ were prepared according to reported procedures. The sodium salt $(1,2-$ $\mathrm{Ph}_{2} \mathrm{PC}_{6} \mathrm{H}_{4} \mathrm{SNa}$ ) was prepared by $1,2-\mathrm{Ph}_{2} \mathrm{PC}_{6} \mathrm{H}_{4} \mathrm{SH}$ with sodium hydride in THF solution. NMR spectra were recorded in J. Young tube on Bruker $500\left(500 \mathrm{MHz}\right.$ for ${ }^{1} \mathrm{H}, 126 \mathrm{MHz}$ for ${ }^{13} \mathrm{C}, 202 \mathrm{MHz}$ for ${ }^{31} \mathrm{P}\left\{{ }^{1} \mathrm{H}\right\} \mathrm{NMR}, 160 \mathrm{MHz}$ for ${ }^{11} \mathrm{~B}, 77 \mathrm{MHz}$ for ${ }^{2} \mathrm{H} \mathrm{NMR}$ ) spectrometers at $298 \mathrm{~K}$. The Chemical shifts for ${ }^{1} \mathrm{H}$ and ${ }^{13} \mathrm{C}$ spectra were reported in ppm relative to either the residual solvent peak or tetramethylsilane (TMS) as an internal standard. The ${ }^{31} \mathrm{P}\left\{{ }^{1} \mathrm{H}\right\}$ NMR spectra were referenced to $85 \% \mathrm{H}_{3} \mathrm{PO}_{4}(0 \mathrm{ppm}) . \mathrm{BF}_{3} \cdot \mathrm{OEt}_{2}$ was used as external standard for ${ }^{11} \mathrm{~B}$ NMR. FT-IR spectra were recorded on a PerkinElmer FT-IR Spectrometer Spectrum Two (the range: from 4000 to $450 \mathrm{~cm}^{-1}$ ), and reported for the $v_{\mathrm{CO}}$ region only. Crystallographic data were collected using a Rigaku Oxford Diffraction XtaLAB Synergy diffractometer equipped with a HyPix-6000HE area detector at $173 \mathrm{~K}$ using $\mathrm{Mo} \mathrm{K \alpha}(\lambda=0.71073 \AA)$ or $\mathrm{Cu} \mathrm{K \alpha}(\lambda=1.54184 \AA)$ from PhotonJet micro-focus X-ray Source. Crystal data collection and refinement parameters are summarized in Tables S5. 


\section{Experimental procedures}

\subsection{Synthesis of molybdenum complexes}

Synthesis of $\left(\mathbf{1}(\mathbf{C O})_{2}\right)$. To a stirred solution of $\mathrm{Cp} * \mathrm{Mo}(\mathrm{CO})_{3} \mathrm{Cl}(212 \mathrm{mg}, 0.6 \mathrm{mmol})$ in 15 mL THF was added 1,2- $\mathrm{Ph}_{2} \mathrm{PC}_{6} \mathrm{H}_{4} \mathrm{SNa}(200 \mathrm{mg}, 0.63 \mathrm{mmol})$ in $15 \mathrm{~mL}$ THF. After refluxing for $5 \mathrm{~h}$, the volatile was removed under vacuum, and the residue was extracted with toluene $(30 \mathrm{~mL})$. The resulting toluene solution was concentrated to about $5 \mathrm{~mL}$, layered with hexane and cooled at $-30{ }^{\circ} \mathrm{C}$ to give $\mathbf{1}(\mathrm{CO})_{2}(314 \mathrm{mg}$, yield $90 \%)$ as yellow microcrystals. ${ }^{1} \mathrm{H}$ NMR (500 MHz, $\left.\mathrm{CDCl}_{3}\right) \delta 7.65$ (d, $\left.J=7.8 \mathrm{~Hz}, 3 \mathrm{H}, \mathrm{Ar} H\right), 7.57$ - 7.46 (m, 5H, ArH), $7.41(\mathrm{t}, J=7.0 \mathrm{~Hz}, 1 \mathrm{H}, \operatorname{Ar} H), 7.31$ (t, $J=10.1 \mathrm{~Hz}, 3 \mathrm{H}, \operatorname{Ar} H), 7.05(\mathrm{t}, J=$ $7.2 \mathrm{~Hz}, 1 \mathrm{H}, \mathrm{Ar} H), 6.90$ (t, J=7.2 Hz, 1H, $\mathrm{Ar} H), 1.64$ (s, 15H, $\left.\mathrm{C}_{5}\left(\mathrm{CH}_{3}\right)_{5}\right) .{ }^{13} \mathrm{C}$ NMR $(126$ $\left.\mathrm{MHz} \mathrm{CDCl}_{3}\right) \delta 251.11\left(\mathrm{~d}, J_{\mathrm{P}-\mathrm{C}}=28.98 \mathrm{~Hz}, \mathrm{Mo}-\mathrm{CO}\right), 250.88(\mathrm{~s}, \mathrm{Mo}-\mathrm{CO}) .{ }^{31} \mathrm{P} \mathrm{NMR}(202$ $\left.\mathrm{MHz}, \mathrm{CDCl}_{3}\right) \delta 84.08(\mathrm{~s})$. FT-IR (THF): $v_{\mathrm{CO}}=1940(\mathrm{~s}), 1865(\mathrm{~s}) \mathrm{cm}^{-1}$. Anal. Calcd for: $\mathrm{C}_{30} \mathrm{H}_{29} \mathrm{MoO}_{2} \mathrm{PS} \mathrm{C}, 62.07$; H, 5.04; Found: C, 62.25; H, 5.24.

Synthesis of $\mathbf{1}(\mathbf{N C M e})_{3}\left(\mathbf{B F}_{4}\right)_{2}$. To a stirred solution of $\mathrm{FcBF}_{4}(373 \mathrm{mg}, 1.36 \mathrm{mmol})$ in 15 $\mathrm{mL} \mathrm{CH}{ }_{3} \mathrm{CN}, \mathbf{1}(\mathrm{CO})_{2}(400 \mathrm{mg}, 0.68 \mathrm{mmol})$ in $15 \mathrm{~mL} \mathrm{CH}_{3} \mathrm{CN}$ was added dropwise. The brown red mixture was stirred for $24 \mathrm{~h}$ at $50{ }^{\circ} \mathrm{C}$ and then evaporated to dryness in vacuo. Ferrocene was washed by $10 \mathrm{~mL} n$-hexane for three times to afford $1(\mathrm{NCMe}) 3^{2+}(450 \mathrm{mg}$, yield 80\%). ${ }^{1} \mathrm{H}$ NMR (500 MHz, $\mathrm{CD}_{2} \mathrm{Cl}_{2}$ ) $\delta 7.63(\mathrm{dd}, J=20.2,10.3 \mathrm{~Hz}, 10 \mathrm{H}, \mathrm{Ar} H), 7.55$ (dd, $J=7.7,2.9 \mathrm{~Hz}, 1 \mathrm{H}, \operatorname{Ar} H), 7.32(\mathrm{t}, J=7.6 \mathrm{~Hz}, 1 \mathrm{H}, \operatorname{Ar} H), 7.02(\mathrm{t}, J=7.5 \mathrm{~Hz}, 1 \mathrm{H}, \operatorname{Ar} H)$, $6.73(\mathrm{t}, J=8.0 \mathrm{~Hz}, 1 \mathrm{H}, \mathrm{Ar} H), 2.32$ (d, $\left.J=2.1 \mathrm{~Hz}, 3 \mathrm{H} \mathrm{CH} \mathrm{H}_{3} \mathrm{CN}\right), 1.95(\mathrm{~d}, J=1.4 \mathrm{~Hz}, 6 \mathrm{H}$, $\left.\mathrm{CH}_{3} \mathrm{CN}\right), 1.93\left(\mathrm{~s}, 15 \mathrm{H}, \mathrm{C}_{5}\left(\mathrm{CH}_{3}\right)_{5}\right) .{ }^{31} \mathrm{P}$ NMR $\left(202 \mathrm{MHz}, \mathrm{CD}_{2} \mathrm{Cl}_{2}\right) \delta 56.33$ (s). Anal. Calcd for: $\mathrm{C}_{34} \mathrm{H}_{38} \mathrm{~B}_{2} \mathrm{~F}_{8} \mathrm{MoN}_{3} \mathrm{PS}$ C, 49.72; H, 4.66; Found: C, 49.75; H, 4.68.

Synthesis of $\mathbf{1}\left(\eta^{2}-\mathrm{NCMe}\right)$. To a stirred solution of $\mathbf{1}\left(\mathrm{NCMe}_{3}\left(\mathrm{BF}_{4}\right)_{2}(460 \mathrm{mg}, 0.56 \mathrm{mmol})\right.$ in $15 \mathrm{~mL} \mathrm{CH}{ }_{3} \mathrm{CN}$ in a Schlenk flask, $\mathrm{Cp}_{2} \mathrm{Co}(212 \mathrm{mg}, 1.12 \mathrm{mmol})$ in $15 \mathrm{~mL}$ THF was added dropwise. In the reaction process, the dark solution turned to red immediately and evaporated to dryness in vacuo. The residue was extracted with toluene and collected as red powder (297 mg, 94\% yield). ${ }^{1} \mathrm{H}$ NMR $\left(500 \mathrm{MHz}, \mathrm{C}_{6} \mathrm{D}_{6}\right) \delta 7.90(\mathrm{t}, J=6.7 \mathrm{~Hz}, 1 \mathrm{H}$, $\operatorname{Ar} H), 7.86(\mathrm{dd}, J=7.7,1.7 \mathrm{~Hz}, 1 \mathrm{H}, \operatorname{Ar} H), 7.47$ (s, 2H, $\operatorname{Ar} H), 7.14-6.97$ (m, 6H, ArH), 
$6.85(\mathrm{ddd}, J=11.3,10.3,4.3 \mathrm{~Hz}, 2 \mathrm{H}, \operatorname{Ar} H), 6.79$ (t, $J=7.3 \mathrm{~Hz}, 2 \mathrm{H}, \mathrm{Ar} H), 2.07$ (s, 3H, $\left.\mathrm{CH}_{3} \mathrm{CN}\right), 1.62\left(\mathrm{~s}, 15 \mathrm{H}, \mathrm{C}_{5}\left(\mathrm{CH}_{3}\right)_{5}\right) .{ }^{13} \mathrm{C} \mathrm{NMR}\left(126 \mathrm{MHz}, \mathrm{C}_{6} \mathrm{D}_{6}\right) \delta 200.5\left(\mathrm{~d}, J_{\mathrm{P}-\mathrm{C}}=110 \mathrm{~Hz}\right.$, Mo-NCMe), ${ }^{31} \mathrm{P}$ NMR (202 MHz, $\left.\mathrm{C}_{6} \mathrm{D}_{6}\right) \delta 104.50$ (s). Anal. Calcd for: $\mathrm{C}_{30} \mathrm{H}_{32} \mathrm{MoNPS}$ C, 63.71; H, 5.70; Found: C, 63.89; H, 5.76.

Synthesis of $\mathbf{1 H}\left(\mathbf{B H}_{2}\right)$. In a glovebox, $\mathbf{1}\left(\eta^{2}-\mathrm{NCMe}\right)(30 \mathrm{mg}, 0.05 \mathrm{mmol})$ was dissolved in $1 \mathrm{~mL}$ THF, two equiv of $\mathrm{H}_{3} \mathrm{~N} \cdot \mathrm{BH}_{3}(3.1 \mathrm{mg}, 0.1 \mathrm{mmol})$ was added to the solution, then the mixture was stirred at room temperature for $10 \mathrm{~min}$ and evaporated to dryness in vacuo. The solid was extracted with $n$-hexane then the hexane solution was concentrated to 0.2 $\mathrm{mL}$ and cooled at $-30^{\circ} \mathrm{C}$ to give $1 \mathrm{H}\left(\mathrm{BH}_{2}\right)$ as red microcrystals (16 mg, 62\% yield). Alternatively, $\mathbf{1 H}\left(\mathrm{BH}_{2}\right)$ can also be afforded by adding one equiv $\mathrm{BH}_{3} \cdot \mathrm{THF}$ to $\mathbf{1}\left(\eta^{2}-\mathrm{NCMe}\right)$ in THF. ${ }^{1} \mathrm{H}$ NMR (500 MHz, $\left.\mathrm{C}_{6} \mathrm{D}_{6}\right) \delta 7.97-7.90(\mathrm{~m}, 1 \mathrm{H}, \mathrm{ArH}), 7.80(\mathrm{t}, J=8.6 \mathrm{~Hz}, 2 \mathrm{H}$, $\operatorname{Ar} H), 7.67-7.57(\mathrm{~m}, 2 \mathrm{H}, \operatorname{Ar} H), 7.31$ (t, $J=6.6 \mathrm{~Hz}, 1 \mathrm{H}, \operatorname{Ar} H), 7.09-7.04$ (m, 2H, ArH), $7.01(\mathrm{dd}, J=10.3,4.1 \mathrm{~Hz}, 3 \mathrm{H}, \operatorname{Ar} H), 6.97-6.92$ (m, 1H, ArH), 6.79 (t, $J=7.4 \mathrm{~Hz}, 1 \mathrm{H}$, $\operatorname{Ar} H), 6.72(\mathrm{t}, J=7.3 \mathrm{~Hz}, 1 \mathrm{H}, \mathrm{Ar} H), 1.80\left(\mathrm{~s}, 15 \mathrm{H}, \mathrm{C}_{5}\left(\mathrm{CH}_{3}\right)_{5}\right),-4.69$ (br, Mo- $\left.H-\mathrm{B}-\mathrm{H}\right),-4.65$ (br, Mo-H-B-H), -7.13 (br, 1H, MoH). ${ }^{31} \mathrm{P}$ NMR (202 MHz, $\mathrm{C}_{6} \mathrm{D}_{6}$ ) $\delta 77.32$ (s). ${ }^{11} \mathrm{~B}$ NMR (160 MHz, $\mathrm{C}_{6} \mathrm{D}_{6}$ ) $\delta$-24.17 (br). Anal. Calcd for: $\mathrm{C}_{28} \mathrm{H}_{32} \mathrm{MoBPS}$ C, 62.47; H, 5.99; Found: C, 62.35; H, 5.78 . 


\subsection{Screening of hydrogen sources of nitriles hydrogenation}

Table S1. Screening of hydrogen sources for nitrile hydrogenation ${ }^{a}$

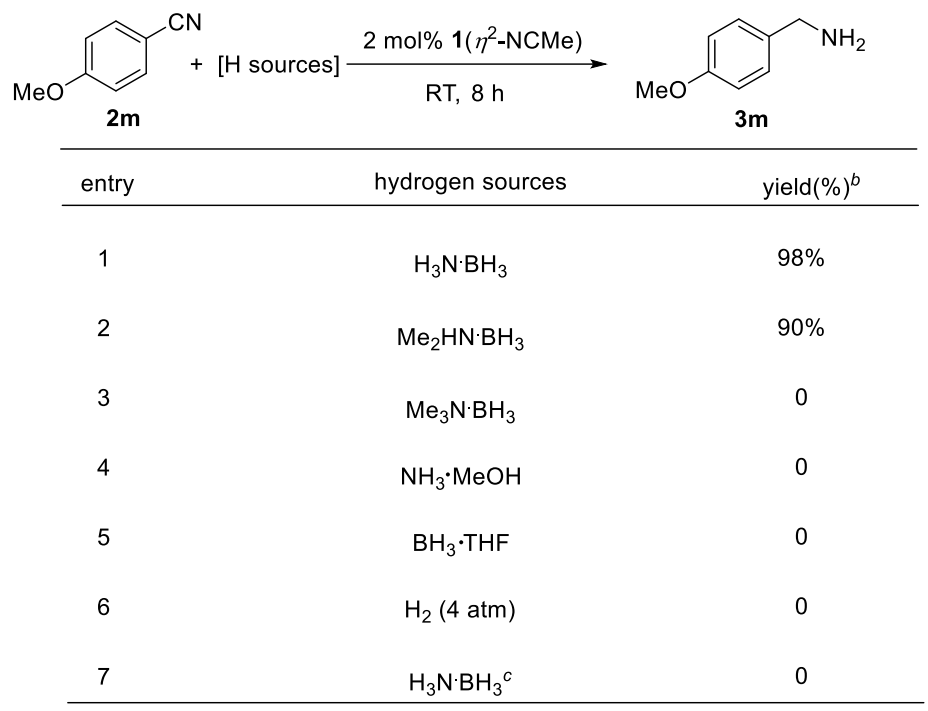

${ }^{a}$ Reaction conditions: nitriles $(66.5 \mathrm{mg}, 0.5 \mathrm{mmol}), \mathrm{H}_{3} \mathrm{~N} \cdot \mathrm{BH}_{3}(31 \mathrm{mg}, 1 \mathrm{mmol}), \mathbf{1}\left(\eta^{2}-\right.$ $\mathrm{NCMe}$ ) (5 mg, $0.01 \mathrm{mmol}, 2 \mathrm{~mol} \%$ ), tetraethylsilane (10 $\mu \mathrm{L}, 0.05 \mathrm{mmol}$, internal standard) in $1 \mathrm{~mL}$ THF. ${ }^{b}$ Yield was determined by ${ }^{1} \mathrm{H}$ NMR based on nitrile. ${ }^{c}$ Without $\mathbf{1}\left(\eta^{2}\right.$-NCMe).

Table S2. Screening of solvent for nitrile hydrogenation ${ }^{a}$

\begin{tabular}{cccc} 
solvent & temperature & NMR yield \\
\hline 1 & hexane & r.t. & $70 \%$ \\
2 & toluene & r.t. & $95 \%$ \\
3 & $\mathrm{CH}_{3} \mathrm{OH}$ & r.t. & $90 \%$ \\
4 & $\mathrm{THF}$ & r.t. & $98 \%$ \\
5 & $\mathrm{HFIP}$ & r.t. & 0 \\
\hline
\end{tabular}

${ }^{a}$ Reaction conditions: nitriles $(66.5 \mathrm{mg}, 0.5 \mathrm{mmol}), \mathrm{H}_{3} \mathrm{~N} \cdot \mathrm{BH}_{3}(31 \mathrm{mg}, 1 \mathrm{mmol}), \mathbf{1}\left(\eta^{2}-\right.$ $\mathrm{NCMe}$ ) (5 mg, $0.01 \mathrm{mmol}, 2 \mathrm{~mol} \%$ ), tetraethylsilane (10 $\mu \mathrm{L}, 0.05 \mathrm{mmol}$, internal standard) in $1 \mathrm{~mL}$ defined solvent. ${ }^{b}$ Yield was determined by ${ }^{1} \mathrm{H}$ NMR based on nitrile. 


\subsection{General procedures for the hydrogenation of nitriles}

Nitriles (0.5 mmol), $\mathrm{H}_{3} \mathrm{~N} \cdot \mathrm{BH}_{3}(32 \mathrm{mg}, 1 \mathrm{mmol})$, tetraethylsilane $(10 \mu \mathrm{L}, 0.05 \mathrm{mmol})$ and $\mathbf{1}\left(\eta^{2}-\mathrm{NCMe}\right)(5 \mathrm{mg}, 0.01 \mathrm{mmol})$ were dissolved in $1 \mathrm{~mL}$ THF in a $10 \mathrm{~mL}$ Schlenk tube, and the mixture was stirred at room temperature for $8 \mathrm{~h}$. Then a sample of this reaction mixture $(100 \mu \mathrm{L})$ was dissolved in $0.5 \mathrm{~mL}$ of $\mathrm{CDCl}_{3}$ and yield of the reaction were determined by ${ }^{1} \mathrm{H}$ NMR signals of the products compared to internal standard (see Figure S1-S2). After that the reaction mixture was filtered through celite, to the collected filtrate was added $0.5 \mathrm{~mL}$ (or $1.0 \mathrm{~mL}$ for the hydrogenation of $\mathbf{3 f}$ ) of a $\mathrm{HCl}$ solution $(1.0 \mathrm{M}$ in $\mathrm{Et}_{2} \mathrm{O}$ ), which resulted in a white precipitate immediately. The solid was washed with DCM then extracted with $\mathrm{MeOH}$ and dried to afford the final product.

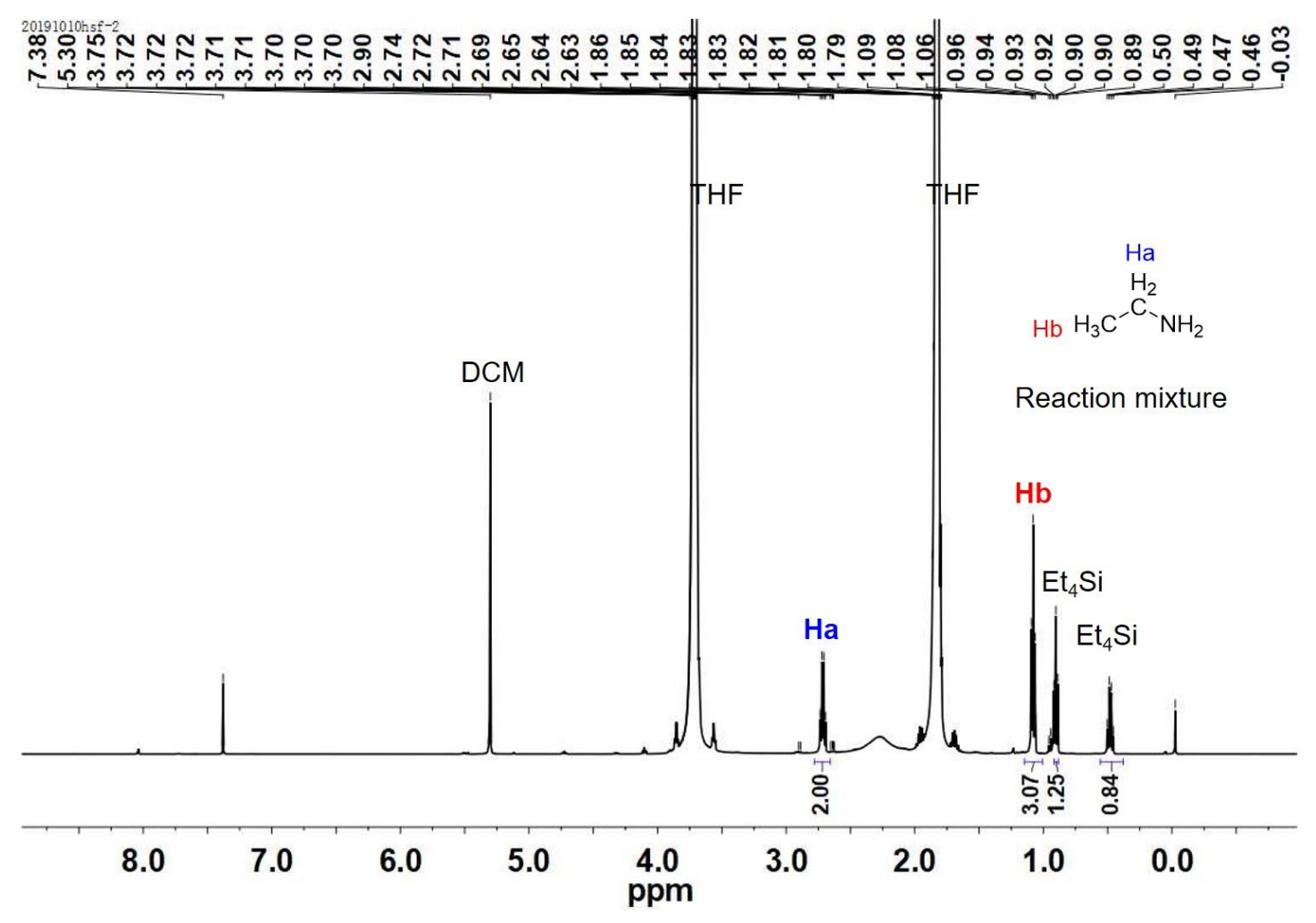

Figure S1. ${ }^{1} \mathrm{H}$ NMR spectrum of the reaction mixture of $\mathbf{3 a}$ in $\mathrm{CDCl}_{3}$. 


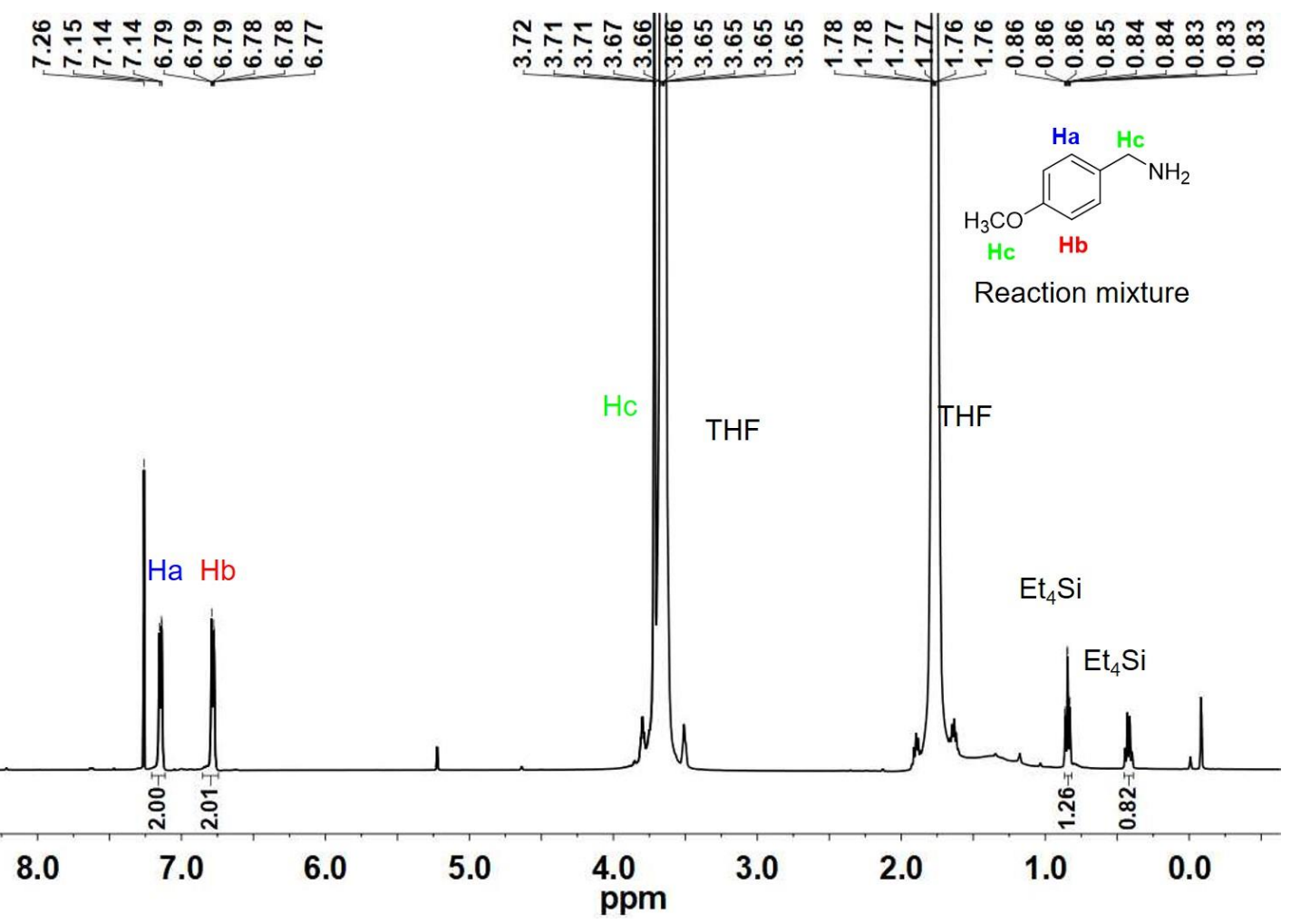

Figure S2. ${ }^{1} \mathrm{H}$ NMR spectrum of the reaction mixture of $\mathbf{3} \mathbf{m}$ in $\mathrm{CDCl}_{3}$. 
Nitriles (0.5 mmol), $\mathrm{H}_{3} \mathrm{~N} \cdot \mathrm{BH}_{3}(32 \mathrm{mg}, 1 \mathrm{mmol})$ and $\mathbf{1}\left(\eta^{2}-\mathrm{NCMe}\right)(5 \mathrm{mg}, 0.01 \mathrm{mmol})$ were dissolved in $1 \mathrm{~mL}$ THF in a $10 \mathrm{~mL}$ Schlenk tube. After $8 \mathrm{~h}$, solvent was removed and the residue was dissolved inTHF- $d 8$ for ${ }^{11} \mathrm{~B}$ NMR, The dehydrogenated products of $\mathrm{H}_{3} \mathrm{~N} \cdot \mathrm{BH}_{3}$ were polyborazylene (PBz), which were identified by their typical chemical shift in ${ }^{11} \mathrm{~B}$ NMR. ${ }^{3}$
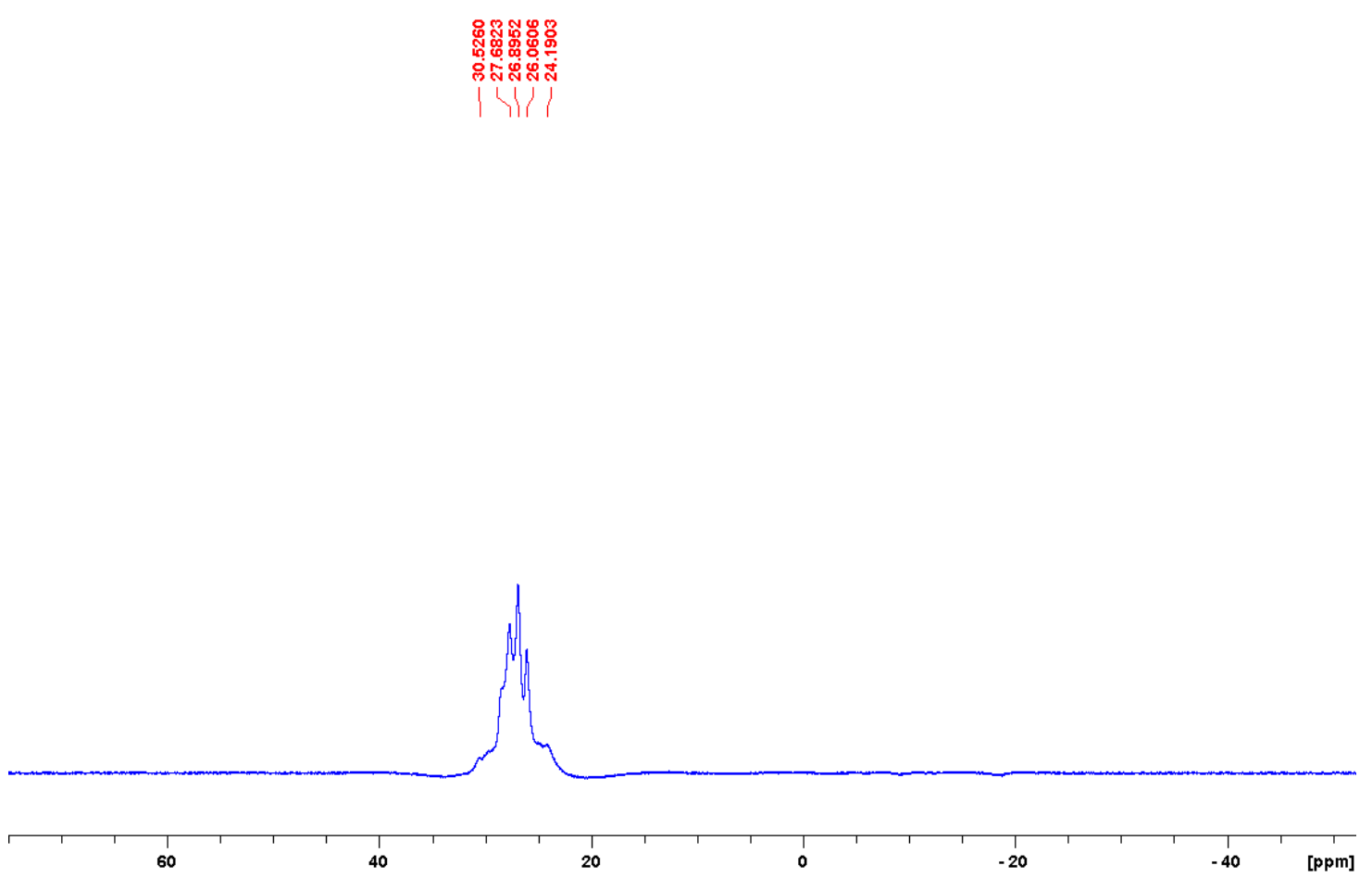

Figure S3. ${ }^{11} \mathrm{~B}$ NMR spectrum of the reaction mixture of $\mathbf{3} \mathbf{m}$ in THF- $d 8$. 


\section{Mechanistic studies}

\subsection{Stoichiometric reaction of $1\left(\eta^{2}-\mathrm{NCMe}\right)$ with $\mathrm{H}_{3} \mathrm{~N} \cdot \mathrm{BH}_{3}$}

$\mathbf{1}\left(\eta^{2}-\mathrm{NCMe}\right)(30 \mathrm{mg}, 0.05 \mathrm{mmol})$ was dissolved in $1 \mathrm{~mL}$ THF- $d 8$, two equiv of $\mathrm{H}_{3} \mathrm{~N} \cdot \mathrm{BH}_{3}$ (3.2 $\mathrm{mg}, 0.1 \mathrm{mmol}$ ) was added to the solution, then the mixture was stirred at room temperature for $10 \mathrm{~min}$. In the ${ }^{31} \mathrm{P}$ NMR spectrum recorded for the reaction mixture, two phosphorus resonances were displayed at $\delta 77.32$ and $\delta 74.73$ as singlets, and only the ${ }^{31} \mathrm{P}$ signal at $\delta 77.32$ was observed after evaporating the solvent and redissolving the residue in THF- $d 8$.

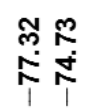

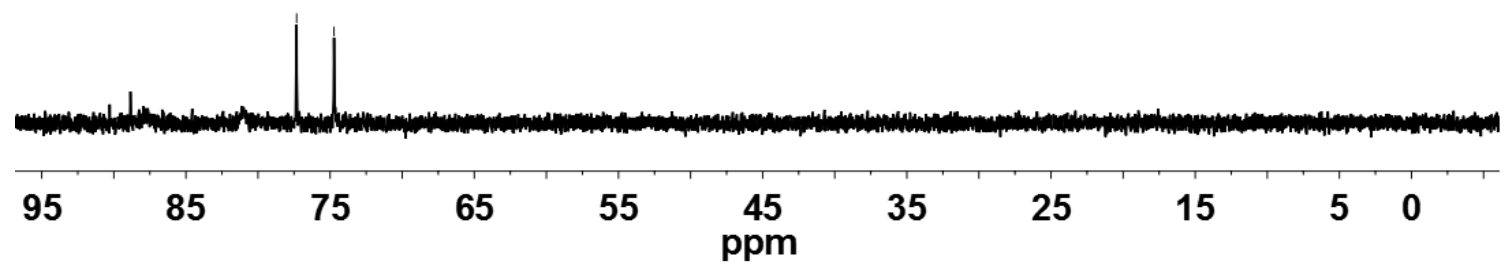

Figure S4. ${ }^{31} \mathrm{P}$ NMR spectrum of the reaction of $\mathbf{1}\left(\eta^{2}-\mathrm{NCMe}\right)$ with $\mathrm{H}_{3} \mathrm{~N} \cdot \mathrm{BH}_{3}$ (2equiv) in THF- $d 8$. 


\section{$\stackrel{\stackrel{m}{*}}{\stackrel{1}{1}}$}

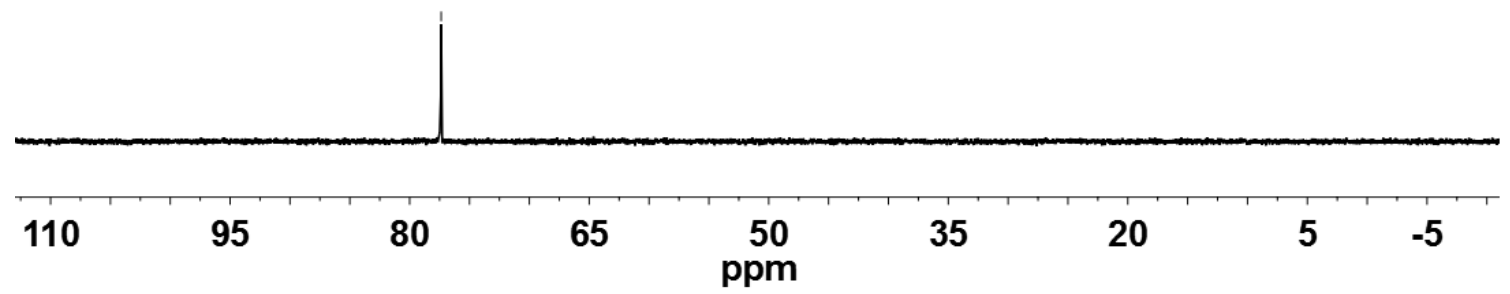

Figure S5. ${ }^{31} \mathrm{P}$ NMR spectrum of the reaction of $\mathbf{1}\left(\eta^{2}-\mathrm{NCMe}\right)$ with $\mathrm{H}_{3} \mathrm{~N} \cdot \mathrm{BH}_{3}$ (2equiv) after evaporating the solvent and re-dissolving the mixture in THF- $d 8$. 


\subsection{Stoichiometric reaction of $1 \mathrm{H}\left(\mathrm{BH}_{2}\right)$ with 4-Methoxybenzonitrile}

Complex 1H(BH $)(20 \mathrm{mg}, 0.036 \mathrm{mmol})$ and 4-methoxybenzonitrile (4.7 mg, $0.036 \mathrm{mmol})$ were dissolved in $0.5 \mathrm{~mL} \mathrm{C}_{6} \mathrm{D}_{6}$ and then added into a J. Young tube. It can be seen in ${ }^{31} \mathrm{P}$ NMR that no new resonances are formed or shifted even heated to $50{ }^{\circ} \mathrm{C}$ indicating that there is no complex formation between $1 \mathrm{H}\left(\mathrm{BH}_{2}\right)$ and 4-methoxybenzonitrile.

(a)

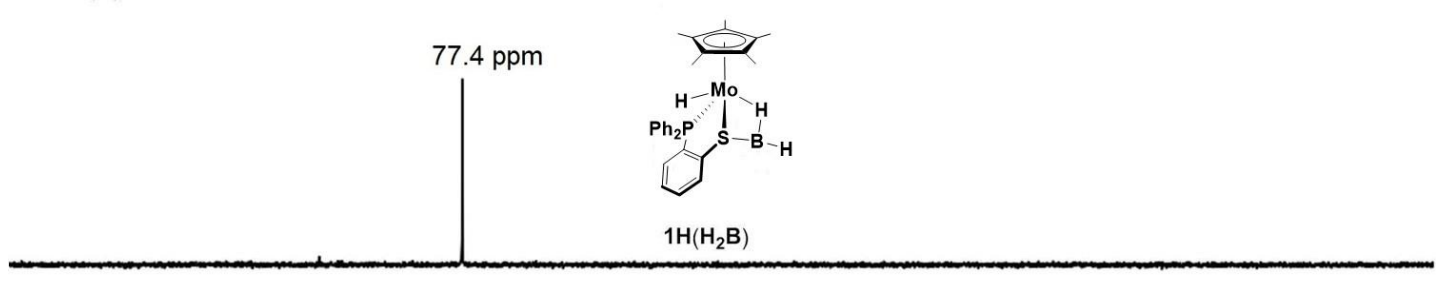

(b)

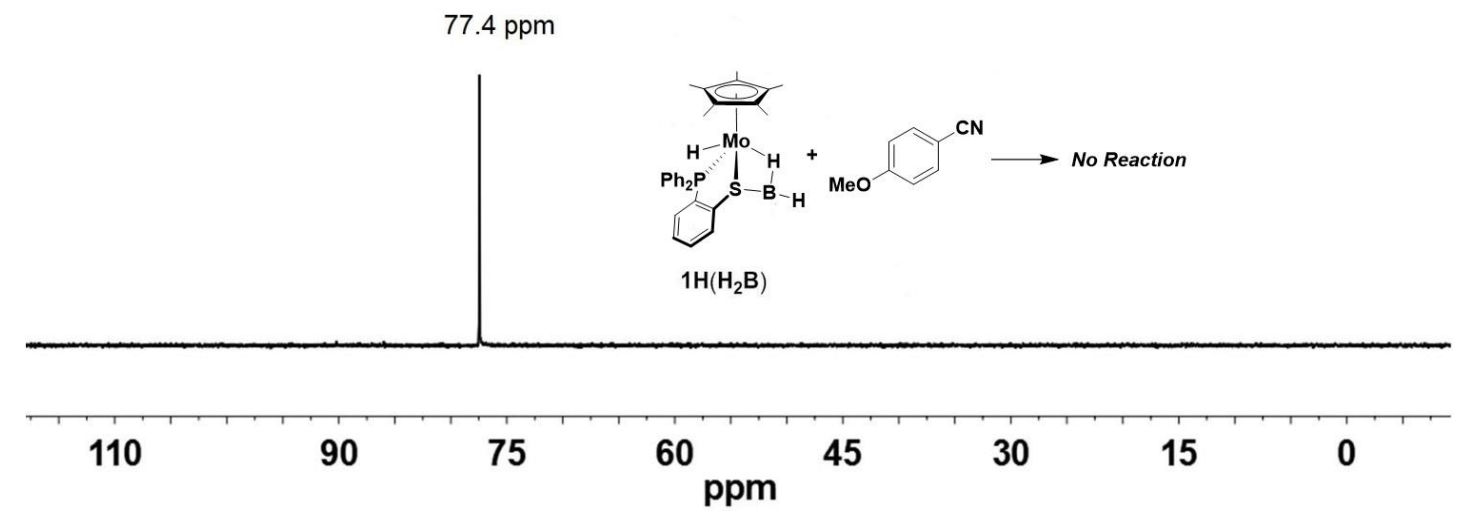

Figure S6. ${ }^{31} \mathrm{P}$ NMR spectra of $1 \mathrm{H}\left(\mathrm{BH}_{2}\right)$ (a) and the reaction of $1 \mathrm{H}\left(\mathrm{BH}_{2}\right)$ with 4methoxybenzonitrile at $50^{\circ} \mathrm{C}$ for $12 \mathrm{~h}(\mathrm{~b})$ in $\mathrm{C}_{6} \mathrm{D}_{6}$. 


\subsection{Stoichiometric reaction of $1 \mathrm{H}\left(\mathrm{BH}_{2}\right)$ with methanol.}

Complex $1 \mathrm{H}\left(\mathrm{BH}_{2}\right)(20 \mathrm{mg}, 0.035 \mathrm{mmol})$ was dissolved in $\mathrm{C}_{6} \mathrm{D}_{6}$, then methanol $(14 \mu \mathrm{L}$, $0.35 \mathrm{mmol}$ ) was added to the solution. After $2 \mathrm{~h}$ at room temperature, no new resonances was observed in ${ }^{31} \mathrm{P}$ NMR spectrum, indicating that there is no complex formation between $1 \mathrm{H}\left(\mathrm{BH}_{2}\right)$ and methanol.

(a)

$$
77.4 \mathrm{ppm}
$$

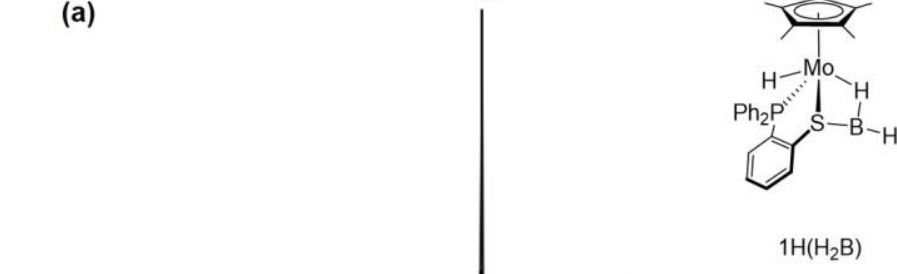

(b)

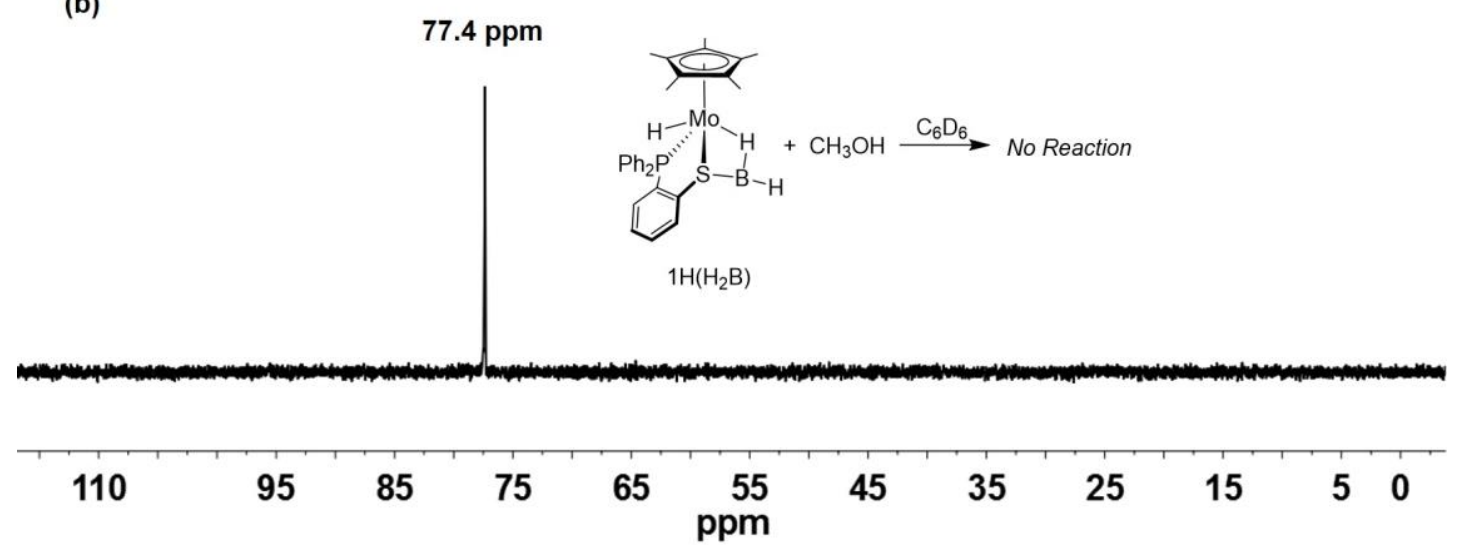

Figure S7. ${ }^{31} \mathrm{P}$ NMR spectra of $1 \mathrm{H}\left(\mathrm{BH}_{2}\right)$ (a), and the reaction mixture of $1 \mathrm{H}\left(\mathrm{BH}_{2}\right)$ with $\mathrm{CH}_{3} \mathrm{OH}(\mathrm{b})$ in $\mathrm{C}_{6} \mathrm{D}_{6}$. 


\subsection{Stoichiometric reaction of $1 \mathrm{H}\left(\mathrm{BH}_{2}\right)$ with $\mathrm{NH}_{3}$}

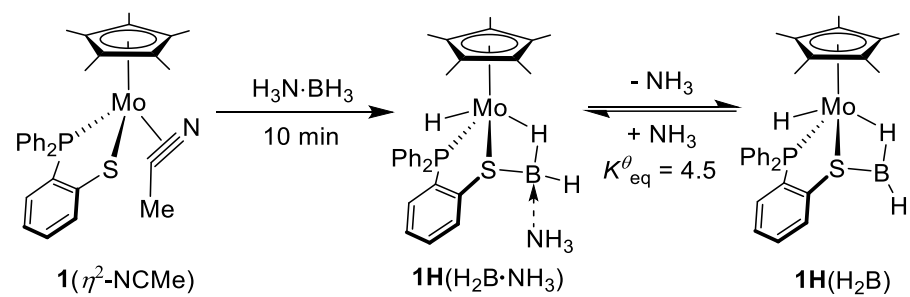

Besides $1 \mathrm{H}\left(\mathrm{BH}_{2}\right)$, an organomolybdenum species with a ${ }^{31} \mathrm{P}$ resonance at $\delta 74.7$ was also formed in the reaction of $\mathbf{1}\left(\eta^{2}-\mathrm{NCMe}\right)$ with $\mathrm{H}_{3} \mathrm{~N} \cdot \mathrm{BH}_{3}$. We propose that this species is the adduct of $1 \mathrm{H}\left(\mathrm{BH}_{2}\right)$ and $\mathrm{NH}_{3}, 1 \mathrm{H}\left(\mathrm{H}_{2} \mathrm{~B} \cdot \mathrm{NH}_{3}\right)$, formed by the interaction between the $\mathrm{BH}_{2}$ moiety and the nitrogen atom of $\mathrm{NH}_{3}$ (above). The evidence of the formation of $1 \mathrm{H}\left(\mathrm{H}_{2} \mathrm{~B} \cdot \mathrm{NH}_{3}\right)$ is derived from the ESI-MS analysis (Figure S42), Calcd for $\left[1 \mathrm{H}\left(\mathrm{H}_{2} \mathrm{~B} \cdot \mathrm{NH}_{3}\right)\right.$ $+\mathrm{H}]^{+}$, 558.1447; found, 558.1439.
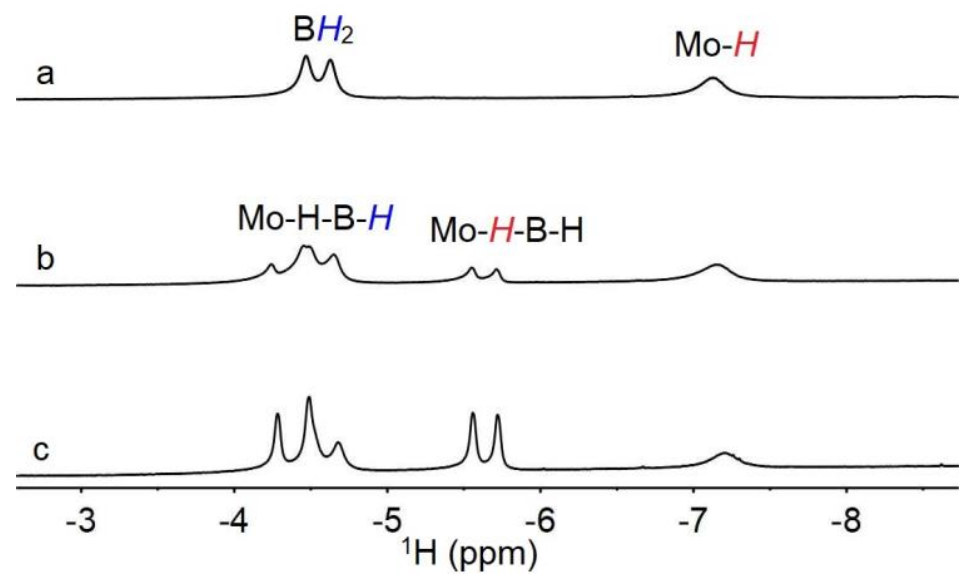

Figure S8. ${ }^{1} \mathrm{H}$ NMR spectra for (a) $1 \mathrm{H}\left(\mathrm{BH}_{2}\right)$, (b) $1 \mathrm{H}\left(\mathrm{BH}_{2}\right)$ with 1 equiv $\mathrm{NH}_{3}(4 \mathrm{M}$ in $\mathrm{MeOH})$ and (c) $\mathbf{1 H}\left(\mathrm{BH}_{2}\right)$ with 2 equiv $\mathrm{NH}_{3}(4 \mathrm{M}$ in $\mathrm{MeOH})$ in $\mathrm{C}_{6} \mathrm{D}_{6}$.

The reaction of $1 \mathrm{H}\left(\mathrm{BH}_{2}\right)$ with $\mathrm{NH}_{3}$ is reversible. Upon addition of a methanol solution of $\mathrm{NH}_{3}$ to $1 \mathrm{H}\left(\mathrm{BH}_{2}\right)$ in $\mathrm{C}_{6} \mathrm{D}_{6}$, the ${ }^{31} \mathrm{P}$ signal in the ${ }^{31} \mathrm{P}$ NMR spectrum at $\delta 74.7$ was reproduced, corresponding to the new ${ }^{11} \mathrm{~B}$ signal shown at $\delta 18.85$ in the ${ }^{11} \mathrm{~B}$ NMR spectrum (Figure S11). In ${ }^{1} \mathrm{H}$ NMR spectrum, the signal for Mo-H terminal hydride 
remained at -7.15 . Two new doublets appeared however at $\delta-4.21\left(J_{\mathrm{B}-\mathrm{H}}=100 \mathrm{~Hz}\right)$ and $5.52\left(J_{\mathrm{B}-\mathrm{H}}=80 \mathrm{~Hz}\right)$, and the peak at $\delta-4.21$ overlaps with the signal at -4.55 for $\mathrm{BH}_{2}$ of $1 \mathrm{H}\left(\mathrm{BH}_{2}\right)$. Galasso and Fronzoni reported that "the B-H coupling constant $J_{\mathrm{B}-\mathrm{H}}$ is very sensitive to the molecular structure, and the bridge H-B coupling constants are often less than $80 \mathrm{~Hz}$ while the $J_{\mathrm{B}-\mathrm{H}}$ constant for terminal B-H bonds falls in the range 90 to 200 $\mathrm{Hz} "{ }^{4}$ Accordingly, the ${ }^{1} \mathrm{H}$ signal at $\delta-4.21$ can be assigned to the terminal hydride Mo$\mathrm{H}-\mathrm{B}-H$ while the ${ }^{1} \mathrm{H}$ signal at $\delta-5.52$ is for the bridging hydrogen, Mo- $H-\mathrm{B}-\mathrm{H}$. 
Complex $1 \mathbf{H}\left(\mathrm{BH}_{2}\right)$ reaction with $\mathbf{N H}_{3}$ (one equivalent). Complex $\mathbf{1} \mathrm{H}_{\left(\mathrm{BH}_{2}\right)}(20 \mathrm{mg}$, $0.035 \mathrm{mmol})$ was dissolved in $\mathrm{C}_{6} \mathrm{D}_{6}(0.5 \mathrm{~mL})$ and transferred to a J. Young tube, followed by the addition of a methanol solution of $\mathrm{NH}_{3}(8.7 \mu \mathrm{L}, 4 \mathrm{M}, 0.035 \mathrm{mmol})$. Then the reaction was monitored by ${ }^{31} \mathrm{P}$ NMR spectroscopy, which indicated that reaction equilibrium had reached in several hours. The ratios of the $1 \mathrm{H}\left(\mathrm{BH}_{2}\right)$ and $1 \mathrm{H}\left(\mathrm{H}_{2} \mathrm{~B} \cdot \mathrm{NH}_{3}\right)$ were determined by integration of ${ }^{31} \mathrm{P}$ NMR spectra at $298 \mathrm{~K}$. At equilibrium, the final relative ratio of $1 \mathrm{H}\left(\mathrm{BH}_{2}\right)$ and $1 \mathrm{H}\left(\mathrm{H}_{2} \mathrm{~B} \cdot \mathrm{NH}_{3}\right)$ was found to be $3.75: 1$ (Figure S9). This value was used to compute $K_{e q}^{\theta}(4.5)$. The free energy change for the reaction $\Delta_{\mathrm{r}} G_{m}^{\theta}$ was -0.89 kcal.

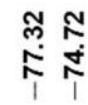

$\stackrel{8}{\circ}$

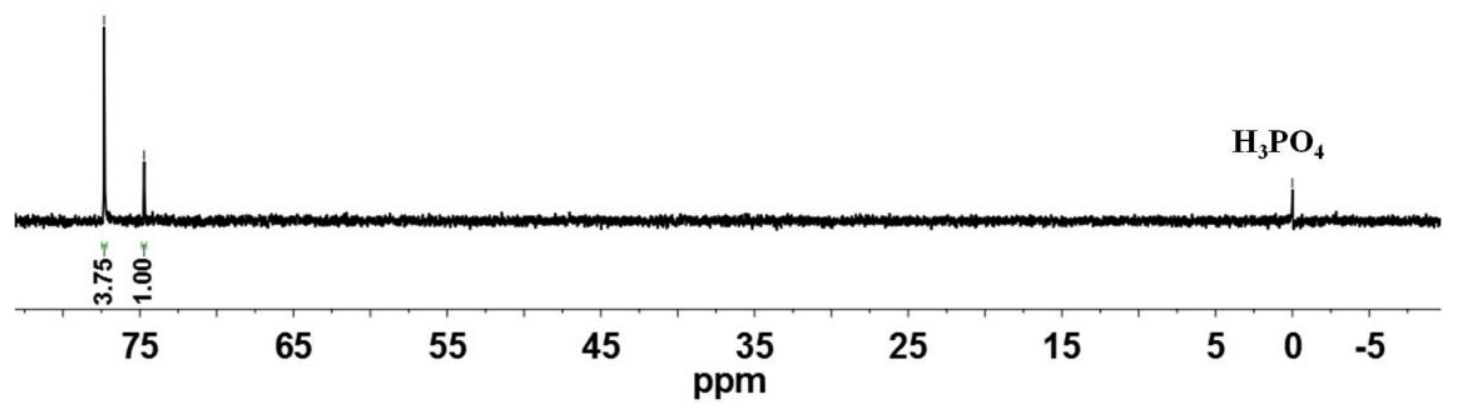

Figure S9. ${ }^{31} \mathrm{P}$ NMR spectrum for $1 \mathrm{H}\left(\mathrm{BH}_{2}\right)$ reaction with $\mathrm{NH}_{3}(\mathrm{MeOH})$ (1 equiv) at equilibrium. The signal at $\delta 0.0$ is the internal standard of $85 \% \mathrm{H}_{3} \mathrm{PO}_{4}$ in capillary. 
Complex $1 \mathbf{H}\left(\mathbf{B H}_{2}\right)$ reaction with $\mathbf{N H}_{3}$ (two equivalent). Complex $\left.\mathbf{1} \mathrm{H}_{(\mathrm{BH}}\right)(20 \mathrm{mg}$,

$0.035 \mathrm{mmol})$ was dissolved in $\mathrm{C}_{6} \mathrm{D}_{6}(0.5 \mathrm{~mL})$ and transferred to a $\mathrm{J}$. Young tube, and a methanol solution of $\mathrm{NH}_{3}(17.4 \mu \mathrm{L}, 0.07 \mathrm{mmol})$ was then added. Then the reaction mixture was monitored by ${ }^{31} \mathrm{P}$ NMR spectroscopy, which indicated equilibrium had reached in several hours. The ratios of the $1 \mathrm{H}\left(\mathrm{BH}_{2}\right)$ and $1 \mathrm{H}\left(\mathrm{H}_{2} \mathrm{~B} \cdot \mathrm{NH}_{3}\right)$ were determined by integration of ${ }^{31} \mathrm{P}$ NMR spectra at $298 \mathrm{~K}$. At equilibrium, the final relative ratio of $1 \mathrm{H}\left(\mathrm{BH}_{2}\right)$ and $1 \mathrm{H}\left(\mathrm{H}_{2} \mathrm{~B} \cdot \mathrm{NH}_{3}\right)$ was found to be 1.90:1 (Figure $\mathrm{S} 10$ ). This value was used to compute $K_{e q}^{\theta}(4.3)$

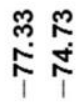

$\stackrel{8}{\substack{i \\ i}}$

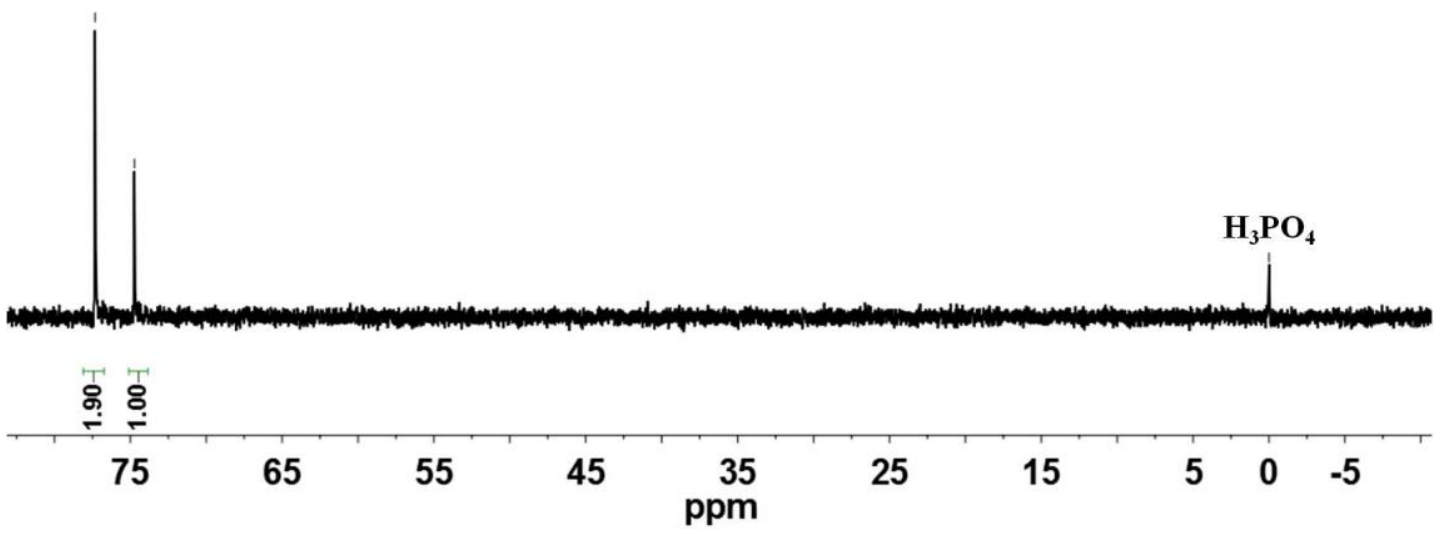

Figure S10. ${ }^{31} \mathrm{P}$ NMR spectrum for $1 \mathrm{H}\left(\mathrm{BH}_{2}\right)$ reaction with $\mathrm{NH}_{3}(\mathrm{MeOH})$ (2 equiv) at equilibrium. The signal at $\delta 0.0$ is the internal standard of $85 \% \mathrm{H}_{3} \mathrm{PO}_{4}$ in capillary. 


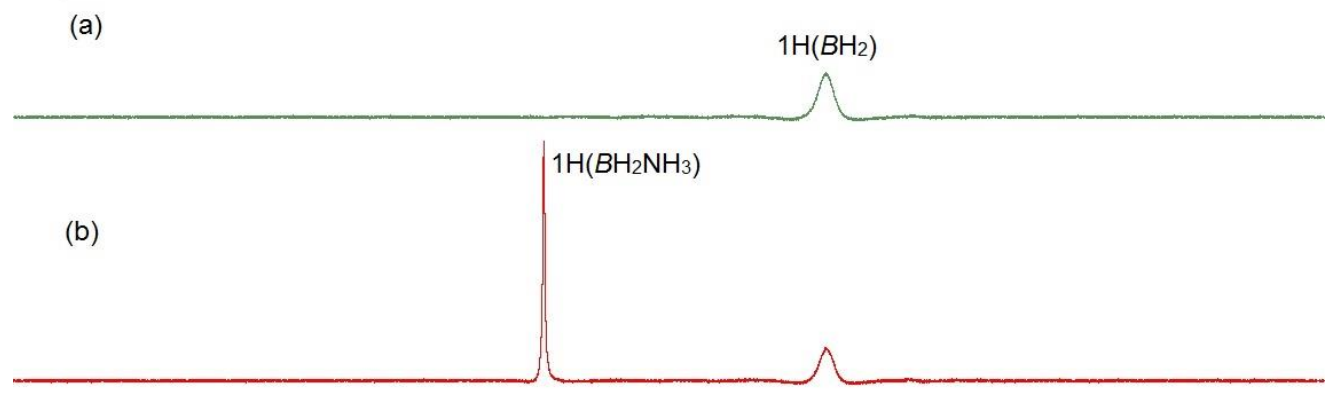

(c)

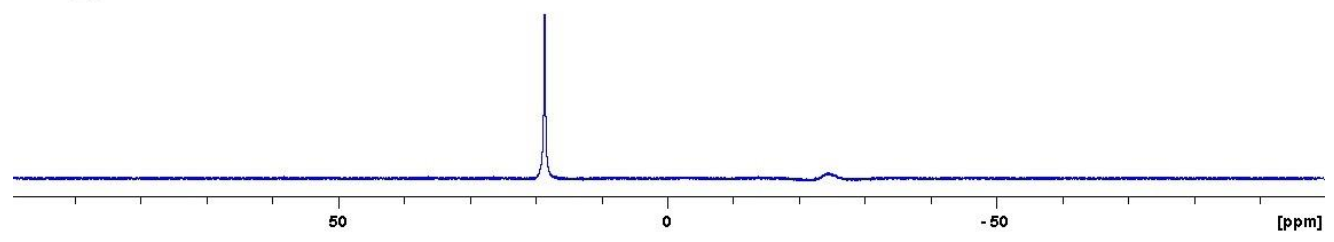

Figure S11. ${ }^{11}$ B NMR spectra for the reactions of (a) $1 \mathrm{H}\left(\mathrm{BH}_{2}\right)$, (b) $1 \mathrm{H}\left(\mathrm{BH}_{2}\right)$ reaction with $\mathrm{NH}_{3}(\mathrm{MeOH})$ (1 equiv) and (c) $\mathbf{1} \mathrm{H}_{\left(\mathrm{BH}_{2}\right)}$ (1 equiv) reaction with $\mathrm{NH}_{3}(\mathrm{MeOH})(2$ equiv).

$1 \mathrm{H}\left(\mathrm{BH}_{2}\right)$ can be completely converted to $1 \mathrm{H}\left(\mathrm{H}_{2} \mathrm{~B} \cdot \mathrm{NH}_{3}\right)$ with excess $\mathrm{NH}_{3}$ solution in $\mathrm{MeOH}$, and $\mathbf{1 H}\left(\mathrm{H}_{2} \mathrm{~B} \cdot \mathrm{NH}_{3}\right)$ is transformed to $\mathbf{1 H}\left(\mathrm{BH}_{2}\right)$ after drying under vacuum. The free energy change $(\Delta G)$ for binding $\mathrm{NH}_{3}$ to $1 \mathrm{H}\left(\mathrm{BH}_{2}\right)$ was estimated from the calculated constant $\left(K_{\text {eq }}^{\theta}=4.4\right)$ to be $-0.875 \mathrm{kcal} / \mathrm{mol}$. The ${ }^{11} \mathrm{~B}$ NMR spectrum of this equilibrium as shown in Figure S11.

Table S3. $K_{\mathrm{eq}}^{\theta}$ and $\Delta_{\mathrm{r}} G_{\mathrm{m}}^{\theta}$ calculation of the reaction of $\mathbf{1 H}\left(\mathrm{BH}_{2}\right)$ with $\mathrm{NH}_{3}(\mathrm{MeOH})$ at equilibrium

$$
K_{e q}^{\theta}=\frac{\frac{c\left[1 \mathrm{H}\left(\mathrm{BH}_{2} \cdot \mathrm{NH}_{3}\right)\right]}{c^{\theta}}}{\frac{c\left[1 \mathrm{H}\left(\mathrm{BH}_{2}\right)\right]}{c^{\theta}} \times \frac{\mathrm{c}\left[\mathrm{NH}_{3}\right]}{c^{\theta}}} \quad \text { (1) } \quad \Delta_{\mathrm{r}} G_{m}^{\theta}=-R T \ln K_{e q}^{\theta}
$$

\begin{tabular}{|c|c|c|c|}
\hline & $\mathbf{1 H}\left(\mathrm{BH}_{2}\right)+\mathrm{NH}_{3}(1$ equiv) & $\mathbf{1 H}\left(\mathrm{BH}_{2}\right)+\mathrm{NH}_{3}$ (2 equiv) & average \\
\hline$K_{e q}^{\theta}$ & 4.5 & 4.3 & 4.4 \\
\hline$\Delta_{\mathrm{r}} G_{m}^{\theta}$ & -0.89 & -0.86 & -0.875 \\
\hline
\end{tabular}




\subsection{Stoichiometric reaction of $1 \mathrm{H}\left(\mathrm{BH}_{2}\right)$ with $\mathrm{NH}_{3}$ and 4-methoxybenzonitrile.}

Complex $1 \mathrm{H}\left(\mathrm{BH}_{2}\right)(20 \mathrm{mg}, 0.035 \mathrm{mmol})$ was dissolved in $\mathrm{C}_{6} \mathrm{D}_{6}$ in a vial, then the methanol solution of $\mathrm{NH}_{3}(8.7 \mu \mathrm{L}, 0.035 \mathrm{mmol})$ was added to the vial. After $0.5 \mathrm{~h}, 4$ methoxybenzonitrile $(4.7 \mathrm{mg}, 0.035 \mathrm{mmol})$ was added to the mixture. The ${ }^{31} \mathrm{P}$ NMR afford a new peak at $\delta 88.4$ (Figure S12). This new species (1A) was proposed to be the molybdenum-thiolate complex coordinated by p-methoxybenzylimine which is from the reduction of $2 \mathrm{~m}$. To verify the structure of this new compound, (4methoxyphenyl)methanimine was synthesized according to the literature. ${ }^{5} \mathrm{In} 1 \mathrm{~mL} \mathrm{THF}$, $\mathbf{1}\left(\eta^{2}-\mathrm{NCMe}\right) \quad(20 \mathrm{mg}, \quad 0.035 \mathrm{mmol}) \quad$ was added to the solution, (4methoxyphenyl)methanimine $(47 \mathrm{mg}, 0.35 \mathrm{mmol})$ was added to the mixture, then the mixture was stirred at room temperature for $5 \mathrm{~h}$. The ${ }^{31} \mathrm{P}$ NMR studies unambiguously reproduced the ${ }^{31} \mathrm{P}$ signal at $\delta 88.41$. In addition, when another equivalent of ammonia borane was added, GC-MS analysis (Figure S13) for the reaction solution indicate the formation of 4-methoxybenzylamine.
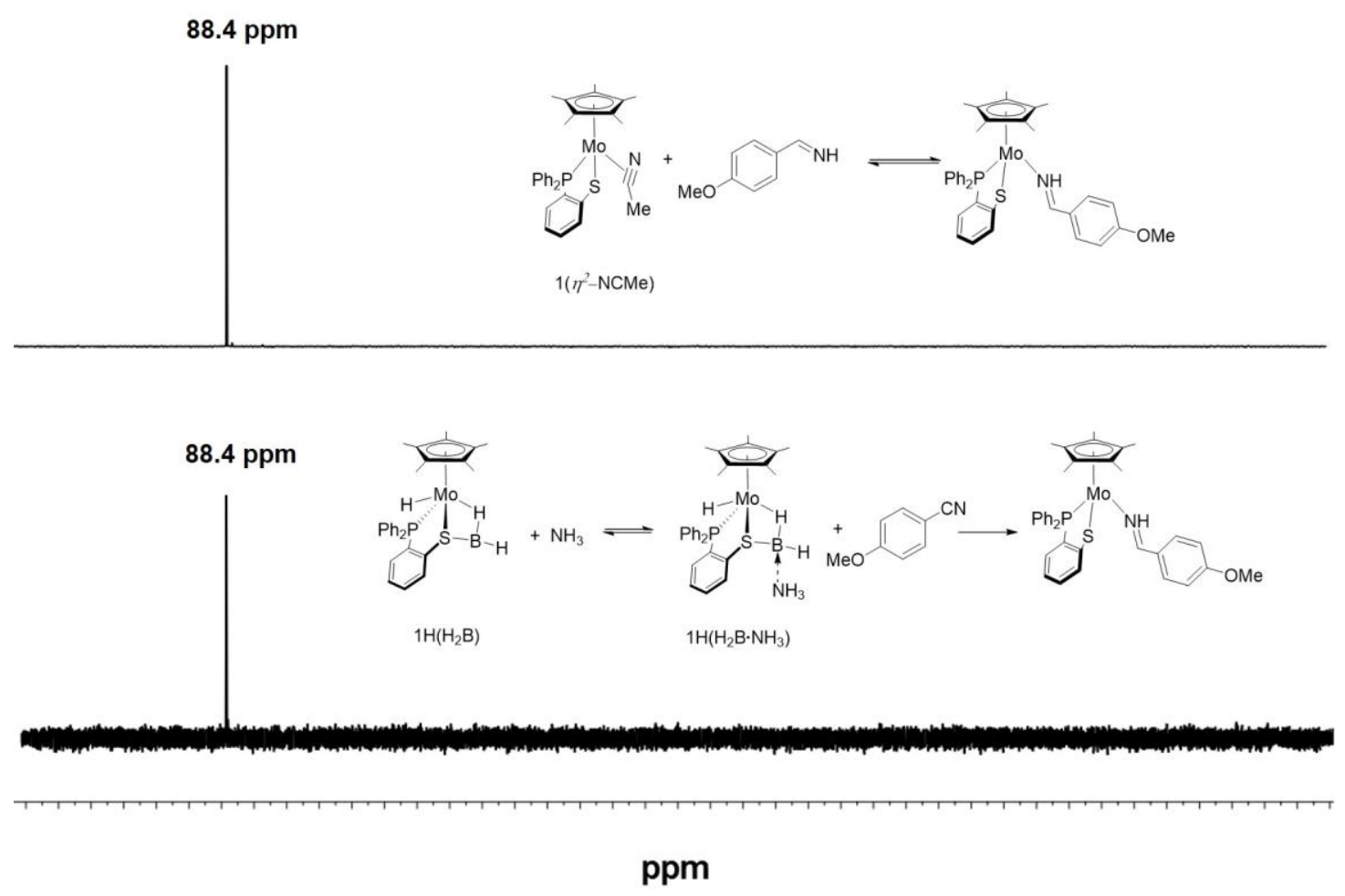
Figure S12. ${ }^{31} \mathrm{P}$ NMR spectra for the reaction between $1\left(\eta^{2}-\mathrm{NCMe}\right)$ with excess (4methoxyphenyl)methanimine(10 equiv) (top); the stoichiometric reaction between $\mathbf{1}\left(\mathrm{BH}_{2}\right), \mathrm{NH}_{3}$ and 4-methoxybenzonitrile (bottom).

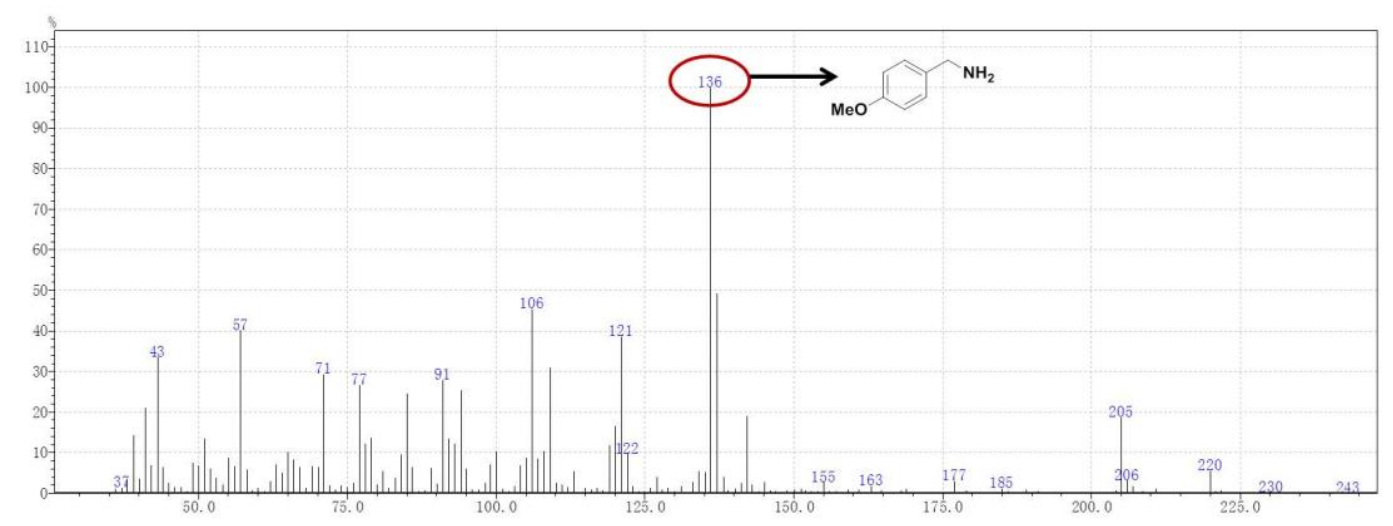

Figure S13. GC-MS spectrum of the reaction of $1 \mathrm{~A}$ with $\mathrm{H}_{3} \mathrm{~N} \cdot \mathrm{BH}_{3}$. 


\subsection{Reaction of imines with ammonia borane.}

In a glove box, (4-methoxyphenyl)methanimine ( $27 \mathrm{mg}, 0.2 \mathrm{mmol})$ was dissolved in 0.5 mL THF in a vial. $\mathrm{H}_{3} \mathrm{~N} \cdot \mathrm{BH}_{3}(6.4 \mathrm{mg}, 0.2 \mathrm{mmol})$ was added to the mixture and stirred at room temperature for $8 \mathrm{~h}$, (4-methoxyphenyl)methanamine was detected by GC-MS.

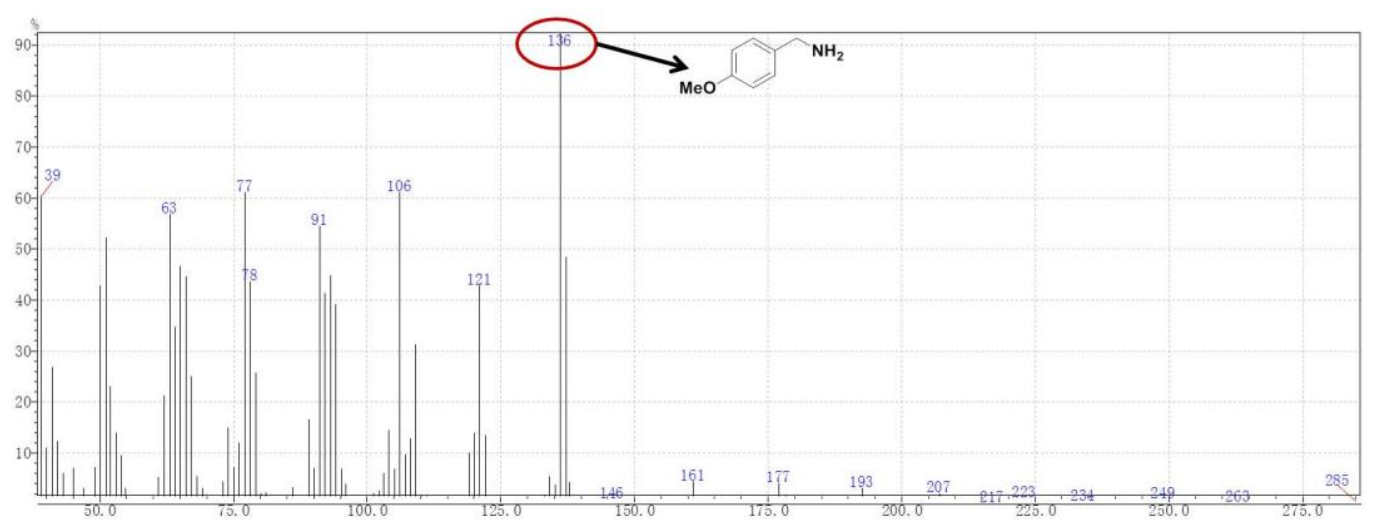

Figure S14. GC-MS spectrum for the reaction of (4-methoxyphenyl)methanimine with $\mathrm{H}_{3} \mathrm{~N} \cdot \mathrm{BH}_{3}$. 


\section{Isotope labelling experiments}

\subsection{Isotope labelling experiments with $\mathrm{D}_{3} \mathrm{~N} \cdot \mathrm{BH}$}

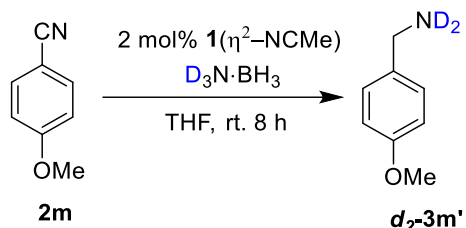

$\mathrm{D}_{3} \mathrm{~N} \cdot \mathrm{BH}_{3}$ and $\mathrm{H}_{3} \mathrm{~N} \cdot \mathrm{BD}_{3}$ were synthesized according to reported literature. ${ }^{6}$ Under the standard conditions $\mathbf{1}\left(\eta^{2}-\mathrm{NCMe}\right)(0.01 \mathrm{mmol}, 5 \mathrm{mg})$ was dissolved in $1 \mathrm{~mL}$ THF in a 10 mL Schlenk tube, after which 4-methoxybenzonitrile $(0.5 \mathrm{mmol}, 66.5 \mathrm{mg})$ and $\mathrm{D}_{3} \mathrm{~N} \cdot \mathrm{BH}_{3}$ $(1 \mathrm{mmol}, 32 \mathrm{mg}$ ) were added to the tube under nitrogen protection. The mixture was stirred at room temperature for 8 hours. After that the reaction mixture was filtered through celite and extracted with dichloromethane. The amine product was characterized by ${ }^{2} \mathrm{H}$ NMR (Figure S15) and ${ }^{1} \mathrm{H}$ NMR analysis (Figure S16). 

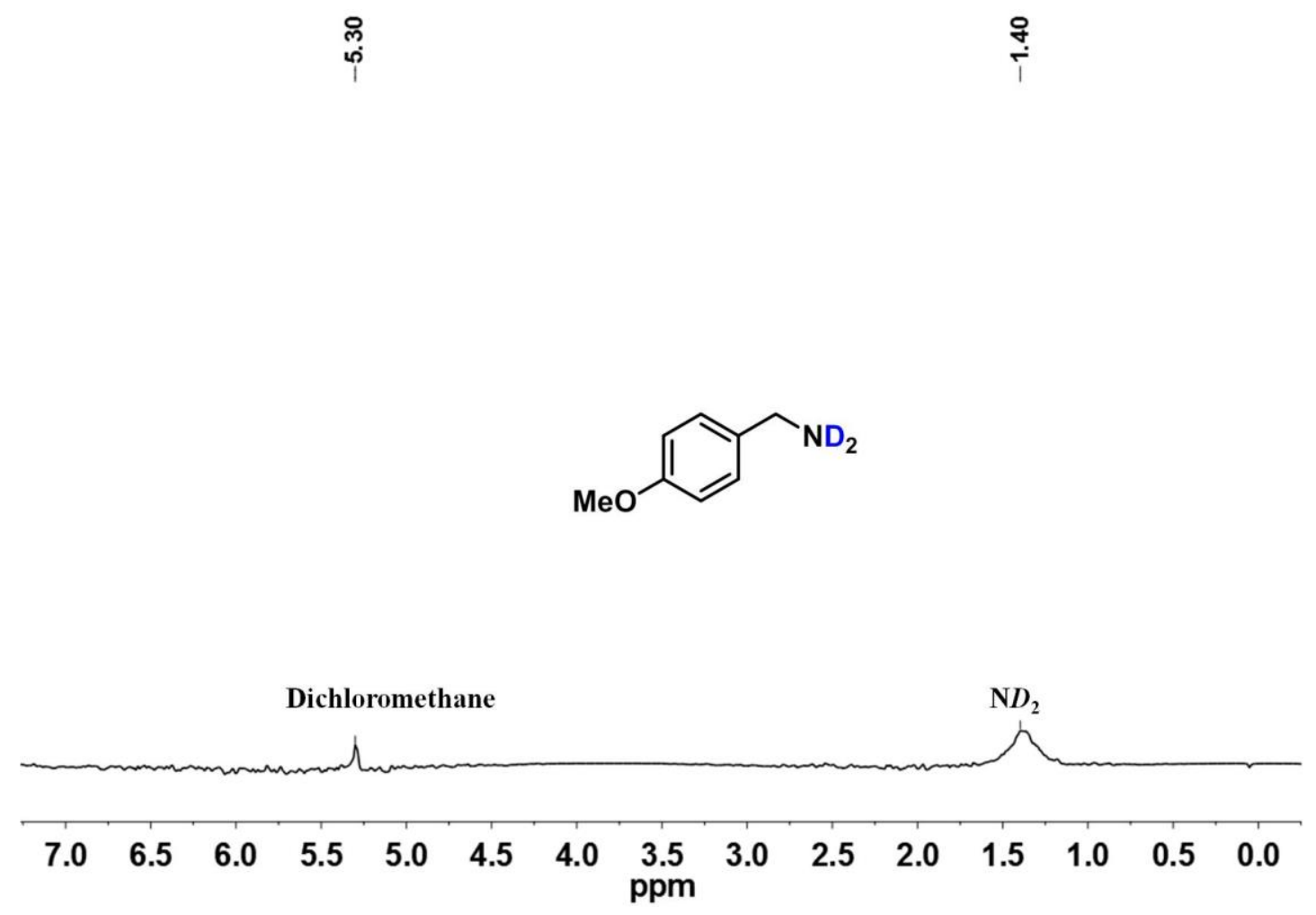

Figure S15. ${ }^{2} \mathrm{H}$ NMR spectrum of $\boldsymbol{d}_{\mathbf{2}} \mathbf{- 3} \mathbf{m}$ ' in $\mathrm{CH}_{2} \mathrm{Cl}_{2}$.

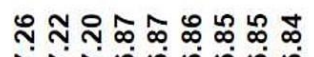

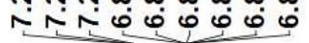

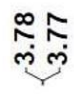

$\stackrel{5}{i}$

匇

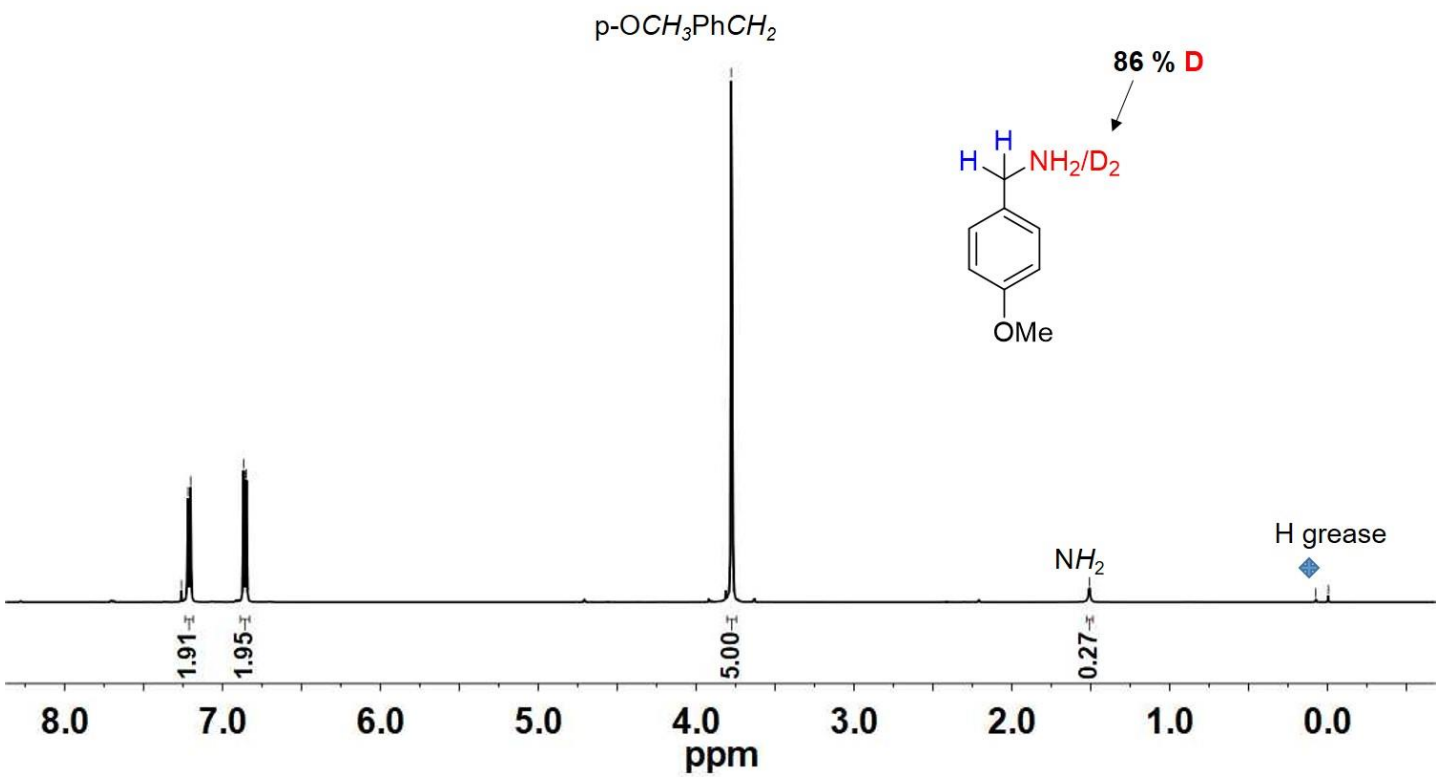

Figure S16. ' ${ }^{1} \mathrm{H}$ NMR spectrum of $\boldsymbol{d}_{\mathbf{2}} \mathbf{- 3} \mathbf{m}$ ' in $\mathrm{CDCl}_{3}$. 


\subsection{Isotope labelling experiments with $\mathrm{H}_{3} \mathrm{~N} \cdot \mathrm{BD}_{3}$}

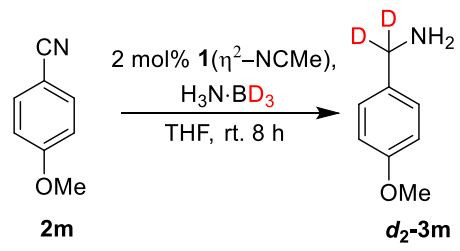

Under the standard conditions $\mathbf{1}\left(\eta^{2}-\mathrm{NCMe}\right)(0.01 \mathrm{mmol}, 5 \mathrm{mg})$ was dissolved in $1 \mathrm{~mL}$ THF in a $10 \mathrm{~mL}$ tube, 4-methoxybenzonitrile $(0.5 \mathrm{mmol}, 66.5 \mathrm{mg})$, and $\mathrm{H}_{3} \mathrm{~N} \cdot \mathrm{BD}_{3}(1 \mathrm{mmol}$, $32 \mathrm{mg}$ ) were added to the tube under nitrogen protection. The mixture was stirred at room temperature for 8 hours. After that the reaction mixture was filtered through celite and extracted with dichloromethane. The amine product was characterized by ${ }^{2} \mathrm{H}$ NMR (Figure S17) and ${ }^{1} \mathrm{H}$ NMR analysis (Figure S18). 


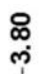

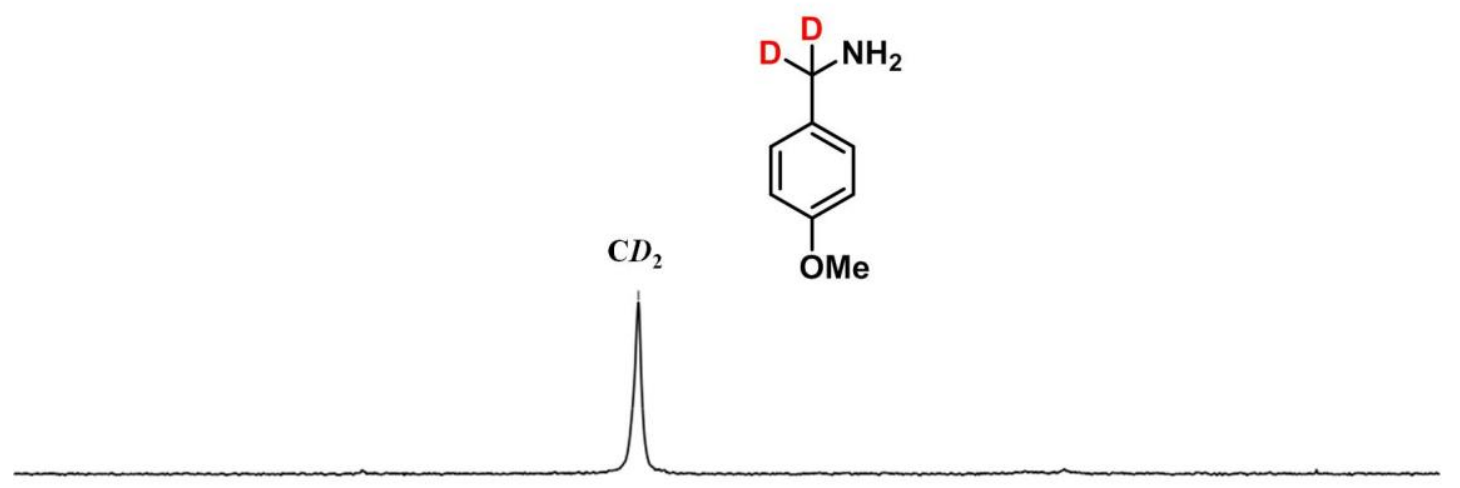

\section{$\begin{array}{lllllllllllllll}7.0 & 6.5 & 6.0 & 5.5 & 5.0 & 4.5 & 4.0 & 3.5 & 3.0 & 2.5 & 2.0 & 1.5 & 1.0 & 0.5 & 0.0\end{array}$} ppm

Figure S17. ${ }^{2} \mathrm{H}$ NMR spectrum of $\boldsymbol{d}_{\mathbf{2}} \mathbf{- 3} \mathbf{m}$ in $\mathrm{CH}_{2} \mathrm{Cl}_{2}$.

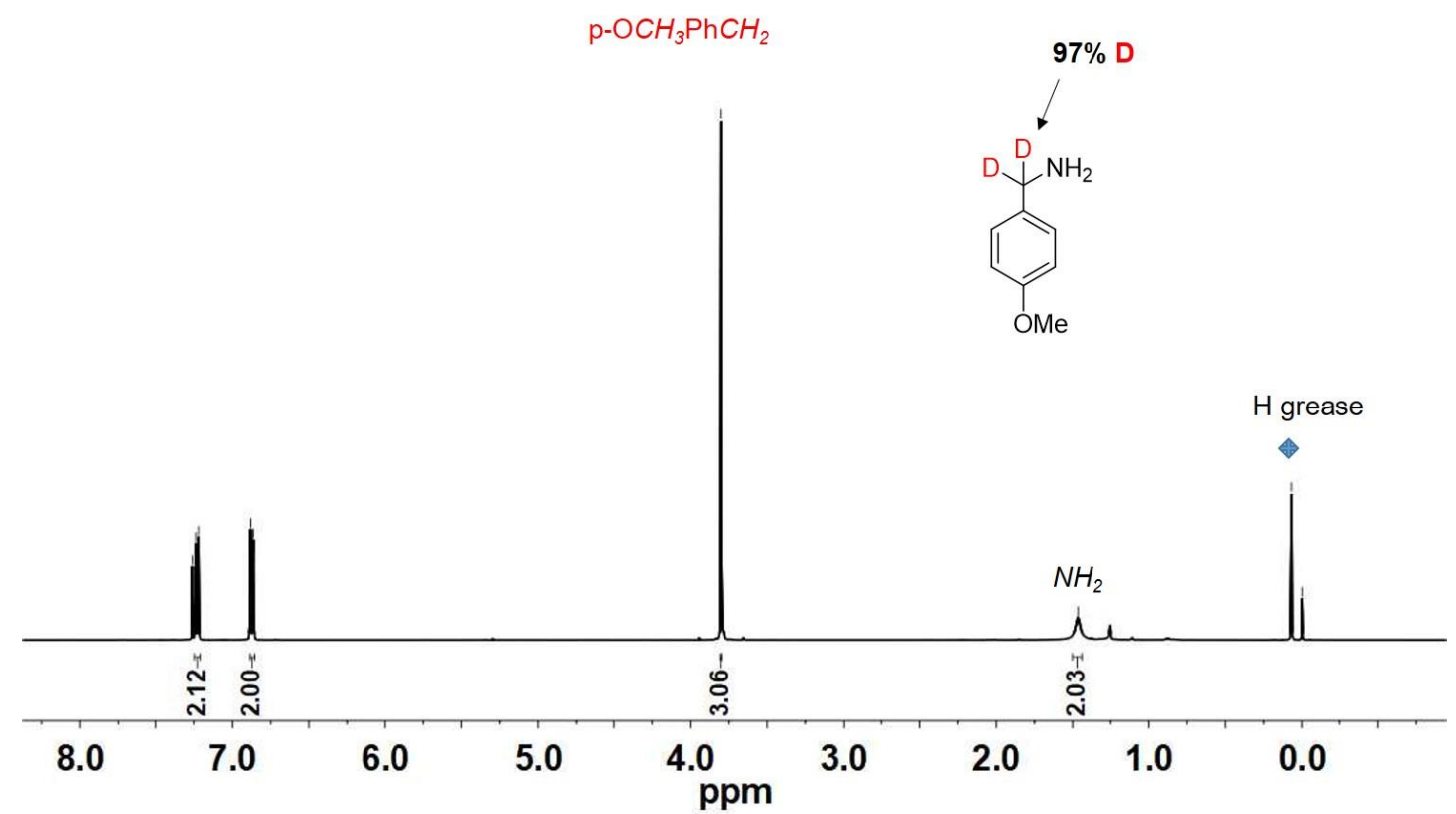

Figure S18. ${ }^{1} \mathrm{H}$ NMR spectrum of $\boldsymbol{d}_{2} \mathbf{- 3} \mathbf{m}$ in $\mathrm{CDCl}_{3}$. 


\section{Kinetic experiments}

The rate law of the reaction was determined using the initial rate methods (up to $\sim 25 \%$ yield). The rate of the reaction correspond to the rate of formation of 4methoxybenzylamine (3m). Kinetic analysis of the NMR-scale reactions were carried out by collecting multiple (10-20) data points in early-stage of the reaction (i.e. the yield of $3 \mathbf{m}<30 \%){ }^{7-10}$ The kinetic data were usually obtained from intensity accumulation in the 4-methoxyphenethylamine proton integral of aromatic ring relative to tetraethylsilane (internal standard) to determine the initial reaction rate. Reaction rates were determined by least-square fit of the initial product concentration versus time $\left(\mathrm{R}^{2}>0.98\right)$.

\section{General method for determination of initial rate}

In a typical experiment, $\mathrm{H}_{3} \mathrm{~N} \cdot \mathrm{BH}_{3}(0.19 \mathrm{mmol}, 5.8 \mathrm{mg})$, 4-Methoxybenzonitrile, $(0.09$ mmol, $12.6 \mathrm{mg})$, and tetraethylsilane $(0.0125 \mathrm{mmol}, 2.5 \mu \mathrm{L}$, internal standard $)$ were added into a vial then $0.5 \mathrm{~mL}$ THF- $d 8$ was injected, then $100 \mu \mathrm{L}$ of solution of complex $1\left(\eta^{2}-\right.$ $\mathrm{NCMe})$ in THF- $d 8(0.0047 \mathrm{M}$ in THF- $d 8)$ was injected by syringe. After all the substrate was added, leading the reaction volume of $600 \mu \mathrm{L}$, the solution was removed to the NMR tube and quickly removed from the glove box. It was shaken, and immediately placed in the pre-adjusted to $298 \mathrm{~K}$ probe of the NMR spectrometer. The reaction was monitored by NMR spectroscopy at $600 \mathrm{~s}$ interval to determine the reaction initial rate. 


\subsection{Complex 1( $\left.\eta^{2}-\mathrm{NCMe}\right)$ rate order assessment}

Varying concentrations of $\mathbf{1}\left(\eta^{2}-\mathrm{NCMe}\right)$ while keeping a constant concentrations of both $\mathrm{H}_{3} \mathrm{~N} \cdot \mathrm{BH}_{3}$ and $\mathbf{2 m}$.

\begin{tabular}{|c|c|c|c|c|}
\hline$\left[\mathrm{H}_{3} \mathrm{~N} \cdot \mathrm{BH}_{3}\right](\mathrm{M})$ & {$[2 \mathrm{~m}](\mathrm{M})$} & {$\left[\mathbf{1}\left(\eta^{2}-\mathrm{NCMe}\right)\right](\mathrm{M})$} & initial rate $(\mathrm{M} / \mathrm{min})$ & $\mathrm{R}^{2}$ \\
\hline 0.32 & 0.16 & 0.0024 & $(7.60 \pm 0.190) \times 10^{-5}$ & 0.9826 \\
\hline 0.32 & 0.16 & 0.0047 & $(1.27 \pm 0.016) \times 10^{-4}$ & 0.9914 \\
\hline 0.32 & 0.16 & 0.0094 & $(2.22 \pm 0.023) \times 10^{-4}$ & 0.9939 \\
\hline 0.32 & 0.16 & 0.0141 & $(3.89 \pm 0.26) \times 10^{-4}$ & 0.9830 \\
\hline
\end{tabular}
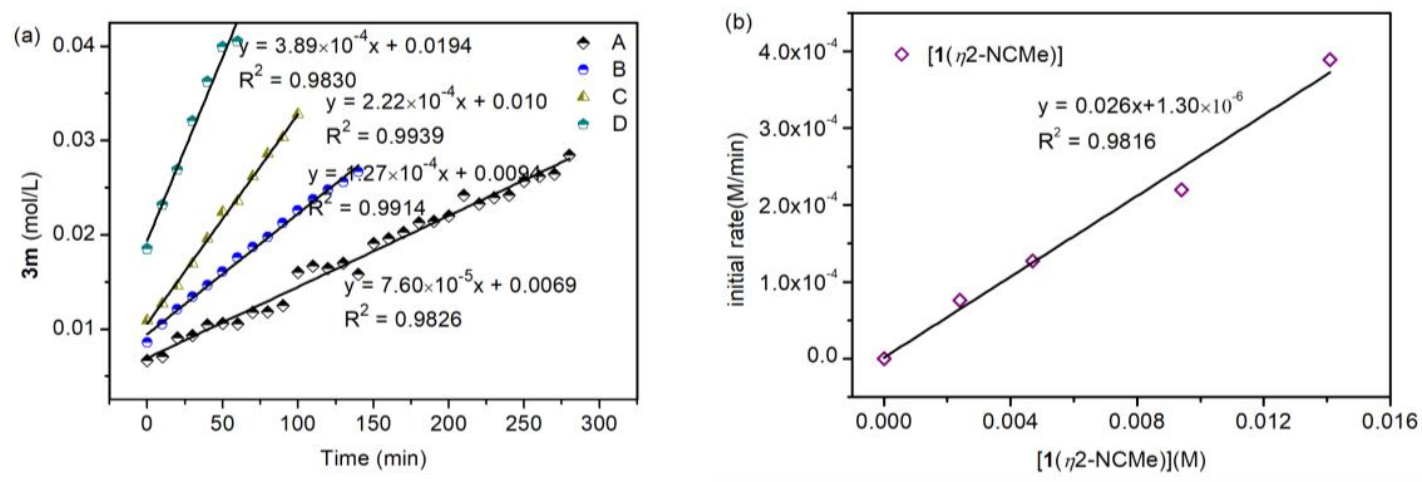

Figure S19. (a) Plot of [3a] against time for varying concentrations of $\mathbf{1}\left(\eta^{2}-\mathrm{NCMe}\right)$, $0.0024 \mathrm{M}(\mathrm{A}), 0.0047 \mathrm{M}(\mathrm{B}), 0.0094 \mathrm{M}(\mathrm{C}), 0.0141 \mathrm{M}(\mathrm{D})$; (b) plot of initial reaction rate $(\mathrm{M} / \mathrm{min})$ vs $\left[\mathbf{1}\left(\eta^{2}-\mathrm{NCMe}\right)\right]$. 


\section{$5.2 \mathrm{H}_{3} \mathrm{~N} \cdot \mathrm{BH}_{3}$ rate order assessment}

Varying concentrations of $\mathrm{H}_{3} \mathrm{~N} \cdot \mathrm{BH}_{3}$ while keeping a constant concentrations of both $\mathbf{1}\left(\eta^{2}-\right.$ $\mathrm{NCMe}$ ) and $\mathbf{2 m}$.

\begin{tabular}{|c|c|c|c|c|}
\hline$\left[\mathrm{H}_{3} \mathrm{~N} \cdot \mathrm{BH}_{3}\right](\mathrm{M})$ & {$[2 \mathrm{~m}](\mathrm{M})$} & {$\left[1\left(\eta^{2}-\mathrm{NCMe}\right)\right](\mathrm{M})$} & initial rate $(\mathrm{M} / \mathrm{min})$ & $\mathrm{R}^{2}$ \\
\hline 0.04 & 0.16 & 0.0047 & $(1.93 \pm 0.03) \times 10^{-5}$ & 0.9931 \\
\hline 0.16 & 0.16 & 0.0047 & $(5.60 \pm 0.11) \times 10^{-5}$ & 0.9926 \\
\hline 0.24 & 0.16 & 0.0047 & $(8.10 \pm 0.12) \times 10^{-5}$ & 0.9967 \\
\hline 0.32 & 0.16 & 0.0047 & $(1.27 \pm 0.02) \times 10^{-4}$ & 0.9914 \\
\hline
\end{tabular}
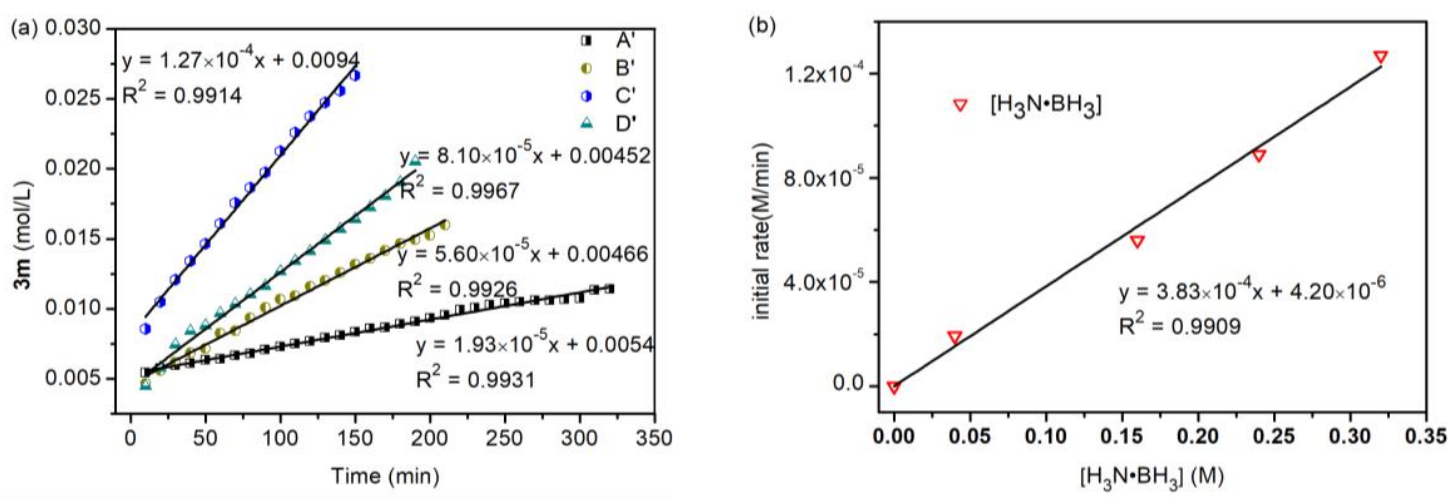

Figure S20. (a) Plot of [3a] against time for varying concentration of $\mathrm{H}_{3} \mathrm{~N} \cdot \mathrm{BH}_{3} 0.04 \mathrm{M}$ (A'), 0.16 M (B'), 0.24 M (C'), 0.32M (D'); (b) plot of reaction initial rate (M/min) vs. $\left[\mathrm{H}_{3} \mathrm{~N} \cdot \mathrm{BH}_{3}\right]$. 


\subsection{4-Methoxybenzonitrile rate order assessment}

Varying concentrations of $2 \mathrm{~m}$ while keeping a constant concentrations of both $\mathrm{H}_{3} \mathrm{~N} \cdot \mathrm{BH}_{3}$ and $\mathbf{1}\left(\eta^{2}-\mathrm{NCMe}\right)$.

\begin{tabular}{|c|c|c|c|c|}
\hline$\left[\mathrm{H}_{3} \mathrm{~N} \cdot \mathrm{BH}_{3}\right](\mathrm{M})$ & {$[2 \mathrm{~m}](\mathrm{M})$} & {$\left[\mathbf{1}\left(\eta^{2}-\mathrm{NCMe}\right)\right](\mathrm{M})$} & initial rate $(\mathrm{M} / \mathrm{min})$ & $\mathrm{R}^{2}$ \\
\hline 0.32 & 0.04 & 0.0047 & $(1.24 \pm 0.22) \times 10^{-4}$ & 0.9914 \\
\hline 0.32 & 0.16 & 0.0047 & $(1.27 \pm 0.02) \times 10^{-4}$ & 0.9951 \\
\hline 0.32 & 0.24 & 0.0047 & $(1.24 \pm 0.03) \times 10^{-4}$ & 0.9894 \\
\hline 0.32 & 0.32 & 0.0047 & $(1.22 \pm 0.15) \times 10^{-4}$ & 0.9952 \\
\hline
\end{tabular}

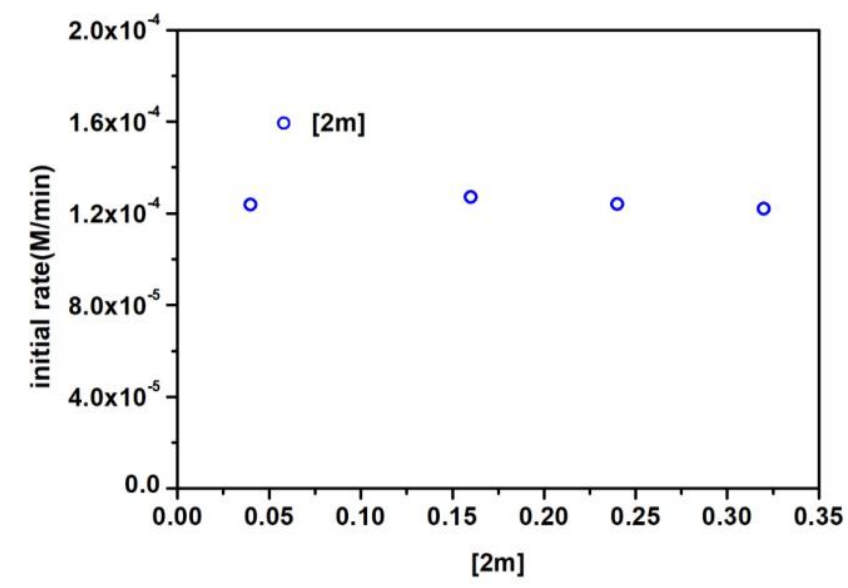

Figure S21. Plot of reaction initial rate $(\mathrm{M} / \mathrm{min})$ vs the concentration of $2 \mathrm{~m}$.

The reaction follows zero order dependence on $2 \mathrm{~m}$ over the probed concentration range. 


\subsection{Determination of the kinetic isotope effect}

KIE was determined using standard condition, data points were collected at $600 \mathrm{~s}$ intervals over $6 \mathrm{~h}$ at $298 \mathrm{~K}$.

\begin{tabular}{|c|c|c|c|}
\hline $\mathbf{1}\left(\eta^{2}-\mathrm{NCMe}\right)$ & $2 \mathrm{~m}$ & $\mathrm{H}_{3} \mathrm{~N} \cdot \mathrm{BH}_{3}$ & tetraethylsilane \\
\hline $0.0028 \mathrm{mmol}$ & $0.096 \mathrm{mmol}$ & $0.19 \mathrm{mmol}$ & $0.0125 \mathrm{mmol}$ \\
$0.0047 \mathrm{M}$ & $0.16 \mathrm{M}$ & $0.32 \mathrm{M}$ & $0.02 \mathrm{M}$ \\
\hline
\end{tabular}

\begin{tabular}{|c|c|c|c|c|}
\hline & $\mathrm{H}_{3} \mathrm{~N} \cdot \mathrm{BH}_{3}$ & $\mathrm{H}_{3} \mathrm{~N} \cdot \mathrm{BD}_{3}$ & $\mathrm{D}_{3} \mathrm{~N} \cdot \mathrm{BH}_{3}$ & $\mathrm{D}_{3} \mathrm{~N} \cdot \mathrm{BD}_{3}$ \\
\hline$k$ & $1.27 \times 10^{-4}$ & $4.96 \times 10^{-5}$ & $9.29 \times 10^{-5}$ & $3.82 \times 10^{-5}$ \\
\hline $\mathrm{KIE}$ & 1 & 2.6 & 1.4 & 3.3 \\
\hline
\end{tabular}

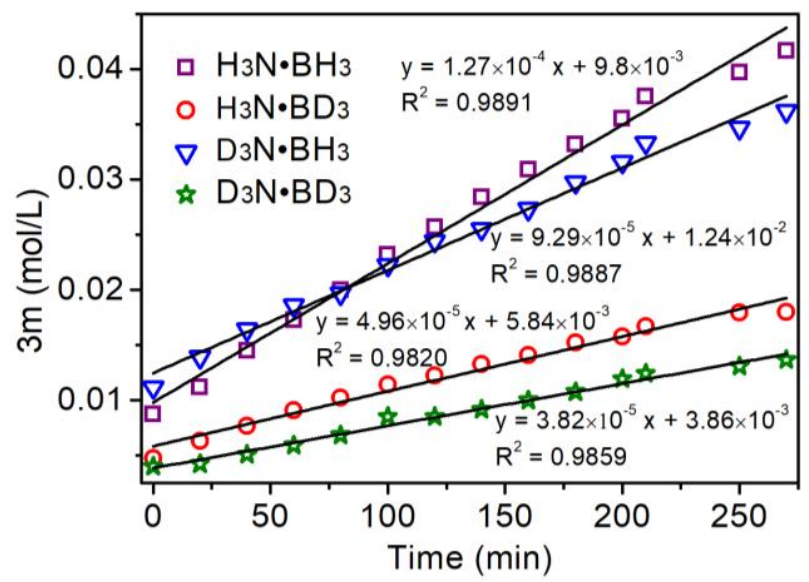

Figure S22. KIE for transfer hydrogenation of $2 \mathrm{~m}$. 


\section{DFT Computational details}

\subsection{Computational details}

The density functional calculations were performed using the Gaussian 16 program package. ${ }^{11}$ Molecular geometries were optimized in the gas phase using the M06-L-D3 functional. ${ }^{12}$ The def2-TZVP basis set (including effective core potential for Mo) ${ }^{13}$ was used for Mo, P, and S, while the def2-SVP basis set was used for all other elements (C, N, and $\mathrm{H}$ ). The final and solvation energies in tetrahydrofuran (THF) were computed as single points on the optimized structures using the SMD continuum solvation model ${ }^{14}$ at both the M06-D3/def2-TZVPP levels. ${ }^{15}$ Analytic frequency calculations were carried out at the same level of theory as for geometry optimization to identify the nature of all stationary points as either minima (no imaginary frequencies) or transition states (only one imaginary frequency), as well as to obtain the thermal Gibbs free energy corrections. A concentration correction of $1.9 \mathrm{kcal} / \mathrm{mol}$ at room temperature was added for all species. ${ }^{16}$

\subsection{Reaction between catalyst $1\left(\eta^{2}-\mathrm{NCMe}\right), \mathrm{CH}_{3} \mathrm{CN}$, and $\mathrm{H}_{3} \mathrm{~N} \cdot \mathrm{BH}_{3}$}

The acetonitrile substrate could bind to the Mo center in an end-on fashion (Complex 2). During the binding, the substrate $\mathrm{C}-\mathrm{N}$ bond increases from $1.16 \AA$ to $1.27 \AA$ (experimental value of $1.25 \AA$, see Table S4), and the C-C-N angle decreases from $180^{\circ}$ to $132.7^{\circ}$ (experimental value of $132.8^{\circ}$ ). 
Table S4. Comparison of experimental and calculated structure of 2.

\begin{tabular}{|c|c|c|c|c|c|}
\hline $\begin{array}{c}\text { selected } \\
\text { bonds }\end{array}$ & $\begin{array}{c}\text { X-ray } \\
(\AA)\end{array}$ & $\begin{array}{c}\text { Calc. }(\AA)- \\
\text { singlet } \\
\end{array}$ & $\begin{array}{c}\text { selected } \\
\text { bonds }\end{array}$ & $\begin{array}{c}\text { X-ray } \\
(\AA)\end{array}$ & $\begin{array}{c}\text { Calc. }(\AA)- \\
\text { singlet }\end{array}$ \\
\hline Mo1-C25 & 2.3482 & 2.3363 & Mo1-N24 & 2.0271 & 2.0055 \\
\hline Mo1-C26 & 2.3470 & 2.3670 & C23-N24 & 1.2460 & 1.2660 \\
\hline Mo1-C27 & 2.3617 & 2.4013 & C22-C23 & 1.4944 & 1.4780 \\
\hline Mo1-C28 & 2.3894 & 2.3821 & P8-C2 & 1.8314 & 1.8235 \\
\hline Mo1-C29 & 2.3616 & 2.3472 & $\mathrm{~S} 21-\mathrm{C} 3$ & 1.7905 & 1.7686 \\
\hline Mo1-P8 & 2.3895 & 2.3877 & P8-C9 & 1.8370 & 1.8324 \\
\hline Mo1-S21 & 2.3780 & 2.3814 & P8-C15 & 1.8427 & 1.8390 \\
\hline Mo1-C23 & 2.0450 & 2.0471 & & & \\
\hline $\begin{array}{l}\text { selected } \\
\text { Angles }\end{array}$ & $\begin{array}{c}\text { X-ray } \\
\left({ }^{\circ}\right)\end{array}$ & $\begin{array}{c}\text { Calc. }\left({ }^{\circ}\right)- \\
\text { singlet }\end{array}$ & $\begin{array}{l}\text { selected } \\
\text { Angles }\end{array}$ & $\begin{array}{c}\text { X-ray } \\
\left({ }^{\circ}\right)\end{array}$ & $\begin{array}{c}\text { Calc. }\left({ }^{\circ}\right)- \\
\text { singlet }\end{array}$ \\
\hline $\begin{array}{l}\text { N24-Mo1- } \\
\text { C23 }\end{array}$ & 35.63 & 36.39 & $\begin{array}{l}\text { Mo1-C23- } \\
\text { C22 }\end{array}$ & 155.20 & 156.22 \\
\hline $\begin{array}{c}\text { N24-C23- } \\
\text { C22 }\end{array}$ & 132.82 & 132.75 & $\begin{array}{l}\text { S21-Mo1- } \\
\text { C23 }\end{array}$ & 94.75 & 95.46 \\
\hline $\begin{array}{l}\text { Mo1-N24- } \\
\text { C23 }\end{array}$ & 72.79 & 73.59 & $\begin{array}{l}\text { S21-Mo1- } \\
\text { N24 }\end{array}$ & 104.44 & 105.28 \\
\hline $\begin{array}{c}\text { Mo1-C23- } \\
\text { N24 }\end{array}$ & 71.40 & 70.02 & P8-Mo1-C25 & 97.62 & 98.35 \\
\hline Mo1-P8-C2 & 106.49 & 106.82 & P8-Mo1-C26 & 94.84 & 95.81 \\
\hline Mo1-S21-C3 & 106.17 & 106.73 & P8-Mo1-C27 & 123.75 & 125.31 \\
\hline $\mathrm{S} 21-\mathrm{C} 3-\mathrm{C} 2$ & 120.64 & 121.00 & P8-Mo1-C28 & 152.48 & 153.95 \\
\hline P8-C2-C3 & 113.07 & 113.02 & P8-Mo1-C29 & 129.27 & 129.68 \\
\hline Mo1-P8-C15 & 118.29 & 119.36 & $\begin{array}{l}\text { S21-Mo1- } \\
\text { C25 }\end{array}$ & 97.78 & 97.22 \\
\hline Mo1-P8-C9 & 120.49 & 120.14 & $\begin{array}{l}\text { S21-Mo1- } \\
\text { C26 }\end{array}$ & 132.35 & 132.19 \\
\hline P8-Mo1-S21 & 79.16 & 79.43 & $\begin{array}{l}\text { S21-Mo1- } \\
\text { C27 }\end{array}$ & 146.28 & 145.22 \\
\hline P8-Mo1-C23 & 81.22 & 79.55 & $\begin{array}{l}\text { S21-Mo1- } \\
\text { C28 }\end{array}$ & 113.96 & 112.50 \\
\hline P8-Mo1-N24 & 116.61 & 115.69 & $\begin{array}{c}\text { S21-Mo1- } \\
\text { C29 }\end{array}$ & 88.72 & 87.48 \\
\hline
\end{tabular}




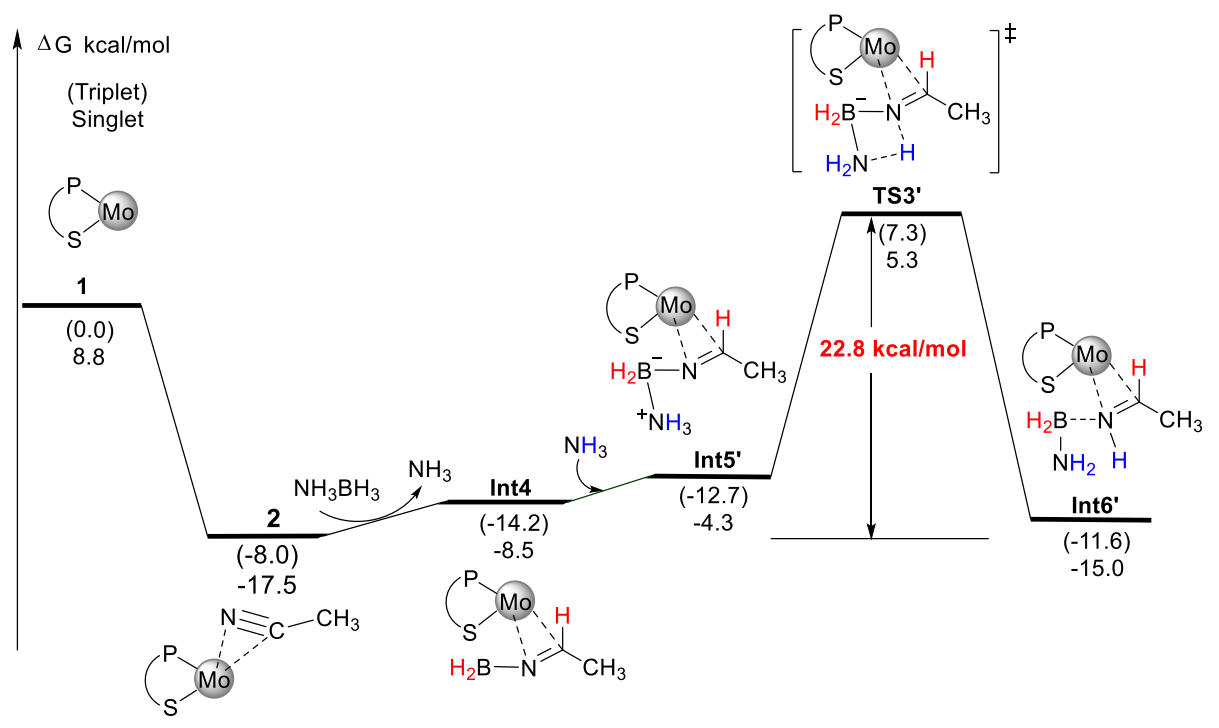

Figure S23. Gibbs energy diagram for the reaction between Int4 and $\mathrm{NH}_{3}$. For the conversion of 2 to Int4, see Figures 6 and 7. 


\subsection{Optimized structures}

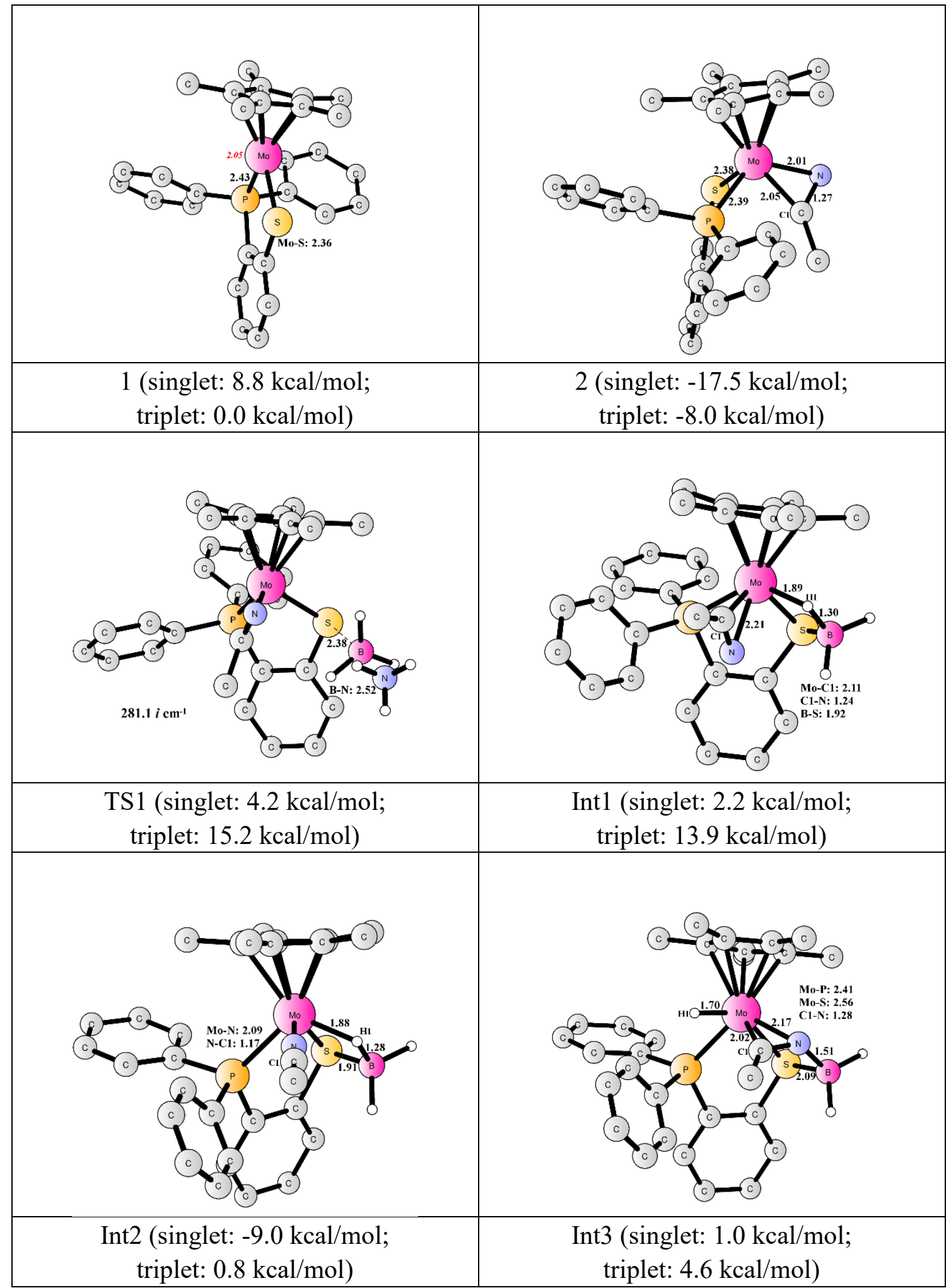




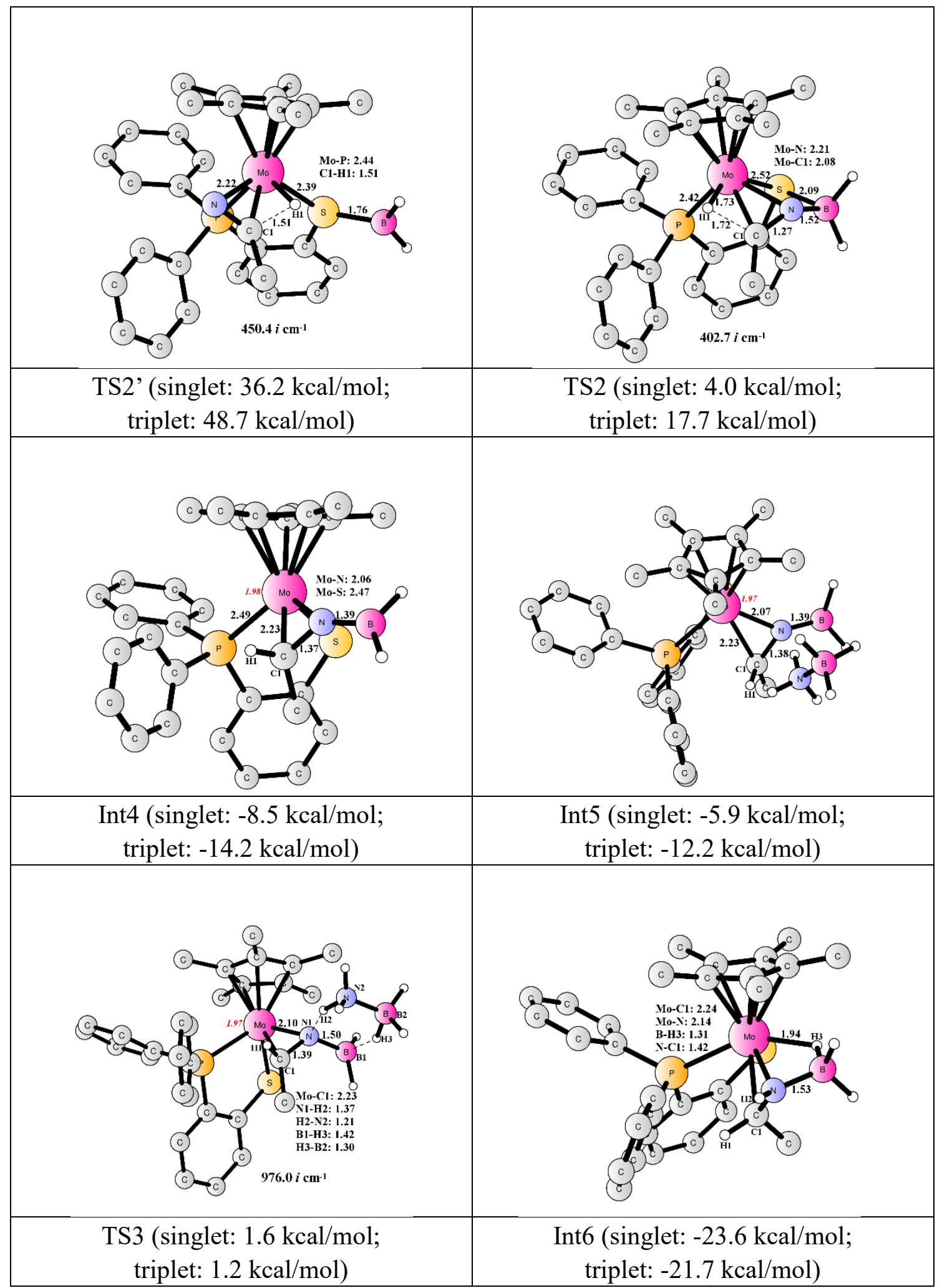




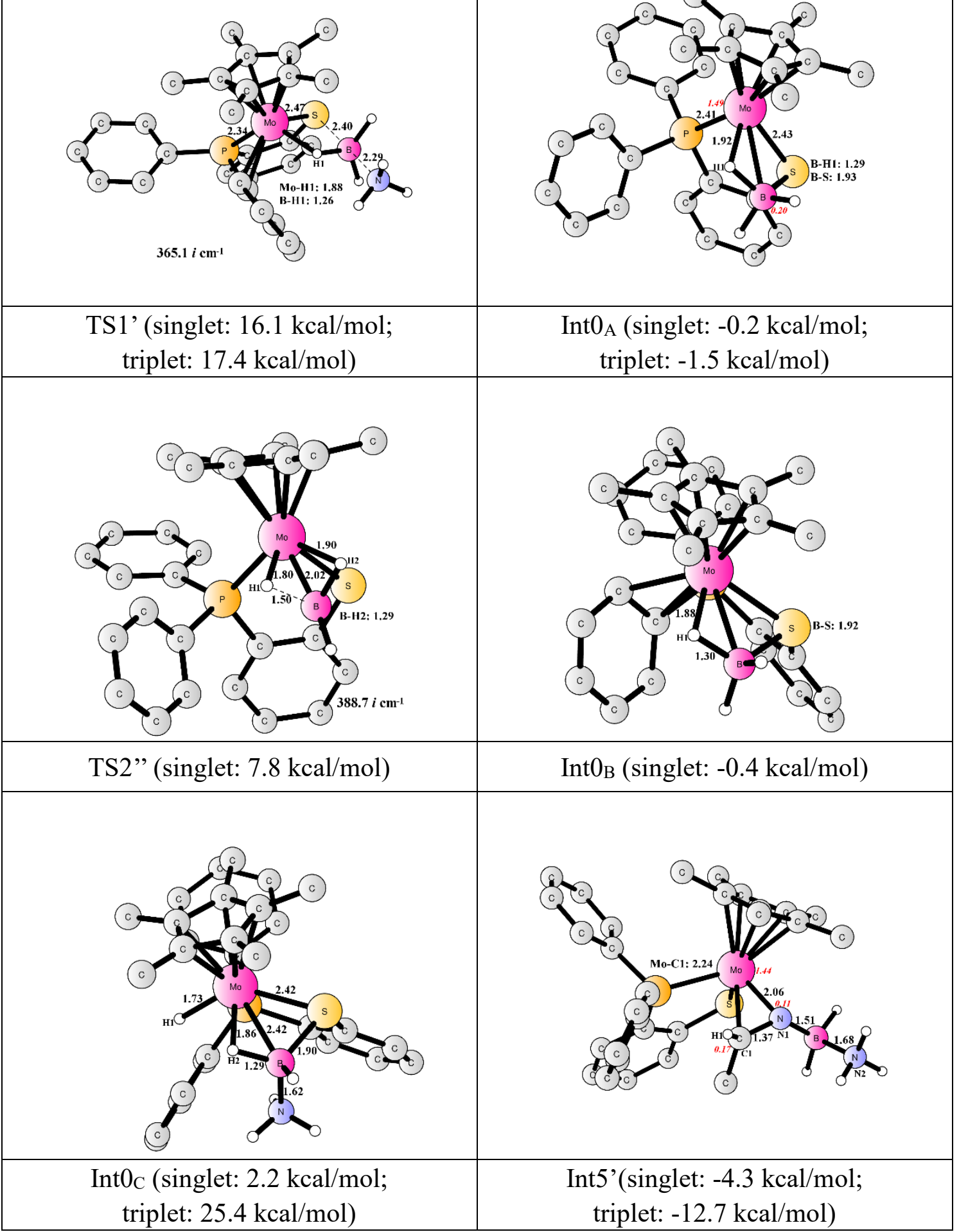




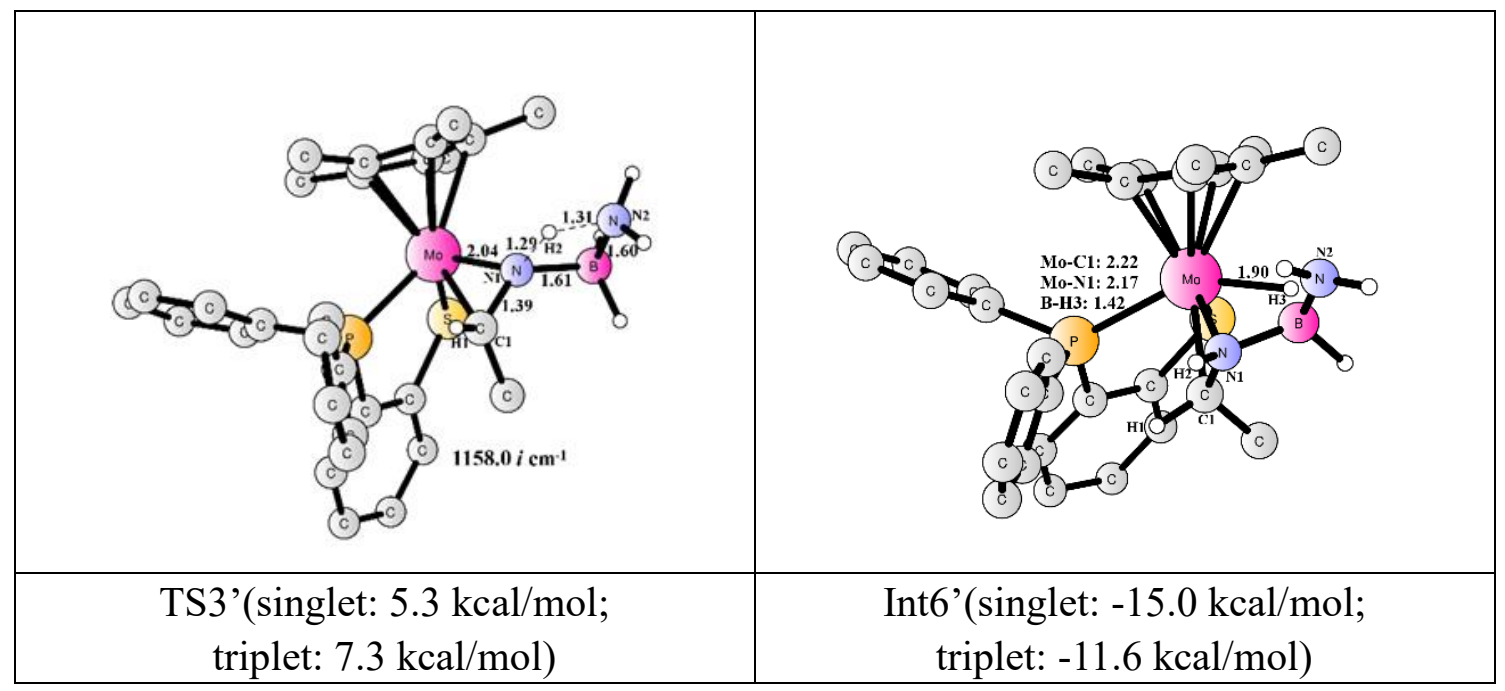




\subsection{Calculated energies in Hartree}

\begin{tabular}{cccc}
\hline & $\begin{array}{c}\text { Geometry optimization } \\
\text { energy }\end{array}$ & $\begin{array}{c}\text { Solvation and large basis } \\
\text { set energy }\end{array}$ & $\begin{array}{c}\text { Gibbs free energy } \\
\text { correction }\end{array}$ \\
\hline 1 & -1891.442948 & -1891.833508 & 0.421561 \\
CH3CN & -132.63741 & -132.7195232 & 0.022208 \\
2 & -2024.153951 & -2024.604736 & 0.470676 \\
NH3BH3 & -83.141074 & -83.2076805 & 0.04754 \\
TS1 & -2107.271365 & -2107.782991 & 0.528556 \\
Int1 & -2050.776366 & -2051.2342 & 0.504164 \\
Int2 & -2050.783361 & -2051.244534 & 0.496684 \\
TS2' & -2050.716278 & -2051.174308 & 0.498495 \\
Int3 & -2050.770849 & -2051.231628 & 0.49968 \\
TS2 & -2050.763945 & -2051.226283 & 0.499084 \\
Int4 & -2050.789892 & -2051.258315 & 0.502099 \\
Int5 & -2133.948485 & -2134.476777 & 0.566541 \\
TS3 & -2133.937537 & -2134.45573 & 0.566865 \\
Int6 & -2051.999275 & -2052.460100 & 0.531041 \\
TS1' & -1974.605491 & -1975.032912 & 0.489504 \\
Int0A & -1918.095335 & -1918.493179 & 0.451531 \\
TS2" & -1918.088903 & -1918.480452 & 0.453619 \\
Int0B & -1918.107752 & -1918.497240 & 0.457458 \\
Int0C & -1974.626173 & -1975.060661 & 0.494749 \\
Int5' & -2107.309217 & -2107.823245 & 0.539566 \\
TS3' & -2107.291454 & -2107.795919 & 0.54092 \\
Int6' & -2107.333412 & -2107.832855 & 0.545458 \\
\hline & & &
\end{tabular}




\section{Coordinates for all structures}

\section{E-1(triplet) opt $=\mathbf{- 1 8 9 1 . 4 4 2 9 4 8 2 h a r t r e e}$}

\begin{tabular}{|c|c|c|c|c|c|}
\hline \multirow{2}{*}{$\begin{array}{l}\text { Center } \\
\text { Number }\end{array}$} & \multirow{2}{*}{$\begin{array}{l}\text { Atomic } \\
\text { Number }\end{array}$} & \multirow{2}{*}{$\begin{array}{l}\text { Atomic } \\
\text { Type }\end{array}$} & \multicolumn{3}{|c|}{ Coordinates (Angstroms) } \\
\hline & & & $\mathrm{X}$ & Y & Z \\
\hline 1 & 42 & 0 & 1.317666 & 0.314640 & -0.347925 \\
\hline 2 & 16 & 0 & 0.566622 & 2.475839 & -0.924553 \\
\hline 3 & 15 & 0 & -1.072300 & -0.035432 & -0.091836 \\
\hline 4 & 6 & 0 & -1.216811 & 2.555176 & -0.947327 \\
\hline 5 & 6 & 0 & -2.008930 & 1.444976 & -0.597754 \\
\hline 6 & 6 & 0 & -3.403408 & 1.535330 & -0.628585 \\
\hline 7 & 6 & 0 & -4.028308 & 2.723560 & -1.001975 \\
\hline 8 & 6 & 0 & -3.245387 & 3.829263 & -1.342754 \\
\hline 9 & 6 & 0 & -1.855743 & 3.749267 & -1.314162 \\
\hline 10 & 6 & 0 & -1.959191 & -1.445543 & -0.823039 \\
\hline 11 & 6 & 0 & -1.417823 & -2.022888 & -1.980165 \\
\hline 12 & 6 & 0 & -2.051745 & -3.095005 & -2.604024 \\
\hline 13 & 6 & 0 & -3.232379 & -3.612072 & -2.070481 \\
\hline 14 & 6 & 0 & -3.777322 & -3.050032 & -0.915797 \\
\hline 15 & 6 & 0 & -3.147562 & -1.971892 & -0.296247 \\
\hline 16 & 6 & 0 & -1.463005 & 1.013079 & 2.471913 \\
\hline 17 & 6 & 0 & -1.369862 & -0.154381 & 1.699318 \\
\hline 18 & 6 & 0 & -1.342022 & -1.398084 & 2.349874 \\
\hline 19 & 6 & 0 & -1.415801 & -1.471428 & 3.739514 \\
\hline 20 & 6 & 0 & -1.504093 & -0.304599 & 4.499960 \\
\hline 21 & 6 & 0 & -1.526818 & 0.935893 & 3.861794 \\
\hline 22 & 6 & 0 & 2.745933 & -0.425916 & 1.183798 \\
\hline 23 & 6 & 0 & 3.517502 & 0.478346 & 0.351373 \\
\hline 24 & 6 & 0 & 3.535870 & -0.062000 & -0.954931 \\
\hline 25 & 6 & 0 & 2.794120 & -1.303728 & -0.942018 \\
\hline 26 & 6 & 0 & 2.348762 & -1.567374 & 0.395168 \\
\hline 27 & 6 & 0 & 4.164868 & 1.737156 & 0.812129 \\
\hline 28 & 6 & 0 & 4.180643 & 0.531938 & -2.158039 \\
\hline 29 & 6 & 0 & 2.633579 & -2.218410 & -2.108709 \\
\hline 30 & 6 & 0 & 1.751008 & -2.834425 & 0.901989 \\
\hline 31 & 6 & 0 & 2.498646 & -0.255147 & 2.645374 \\
\hline 32 & 1 & 0 & -4.011363 & 0.665634 & -0.360036 \\
\hline 33 & 1 & 0 & -5.118216 & 2.787471 & -1.027884 \\
\hline 34 & 1 & 0 & -3.725133 & 4.766068 & -1.638263 \\
\hline 35 & 1 & 0 & -1.245275 & 4.615378 & -1.582491 \\
\hline 36 & 1 & 0 & -0.483537 & -1.620136 & -2.387137 \\
\hline 37 & 1 & 0 & -1.619537 & -3.532420 & -3.507044 \\
\hline
\end{tabular}




\begin{tabular}{rrrrrr}
\hline 38 & 1 & 0 & -3.727469 & -4.457924 & -2.553046 \\
39 & 1 & 0 & -4.699959 & -3.454747 & -0.493046 \\
40 & 1 & 0 & -3.576466 & -1.544370 & 0.614837 \\
41 & 1 & 0 & -1.479591 & 1.988764 & 1.977379 \\
42 & 1 & 0 & -1.264473 & -2.317046 & 1.761475 \\
43 & 1 & 0 & -1.403327 & -2.447232 & 4.231460 \\
44 & 1 & 0 & -1.558291 & -0.362685 & 5.589441 \\
45 & 1 & 0 & -1.597659 & 1.853538 & 4.450435 \\
46 & 1 & 0 & 4.341186 & 2.434585 & -0.017299 \\
47 & 1 & 0 & 3.548870 & 2.268411 & 1.551183 \\
48 & 1 & 0 & 5.139287 & 1.540950 & 1.289903 \\
49 & 1 & 0 & 3.565181 & 0.402560 & -3.059978 \\
50 & 1 & 0 & 4.358970 & 1.608526 & -2.039829 \\
51 & 1 & 0 & 5.153823 & 0.058060 & -2.368067 \\
52 & 1 & 0 & 1.775182 & -2.892787 & -1.983281 \\
53 & 1 & 0 & 2.488163 & -1.671745 & -3.052052 \\
54 & 1 & 0 & 3.523389 & -2.855882 & -2.247718 \\
55 & 1 & 0 & 1.199877 & -2.673534 & 1.838990 \\
56 & 1 & 0 & 1.046038 & -3.278730 & 0.183785 \\
57 & 1 & 0 & 2.521221 & -3.596772 & 1.112972 \\
58 & 1 & 0 & 2.430691 & 0.804512 & 2.930476 \\
59 & 1 & 0 & 1.559376 & -0.732862 & 2.960151 \\
60 & 1 & 3.307289 & -0.698797 & 3.250741 \\
& 1 & 0 & & &
\end{tabular}

\section{$\mathrm{E}-\mathrm{CH}_{3} \mathrm{CN}$ (singlet)opt $=\mathbf{- 1 3 2 . 6 3 7 4 1}$ hartree}

\begin{tabular}{|c|c|c|c|c|c|}
\hline \multirow{2}{*}{$\begin{array}{l}\text { Center } \\
\text { Number }\end{array}$} & \multirow{2}{*}{$\begin{array}{l}\text { Atomic } \\
\text { Number }\end{array}$} & \multirow{2}{*}{$\begin{array}{l}\text { Atomic } \\
\text { Type }\end{array}$} & \multicolumn{3}{|c|}{ Coordinates (Angstroms) } \\
\hline & & & $X$ & $\mathrm{Y}$ & Z \\
\hline 1 & 6 & 0 & 0.000000 & 0.000000 & -1.171313 \\
\hline 2 & 1 & 0 & 0.000000 & 1.026629 & -1.558999 \\
\hline 3 & 1 & 0 & -0.889087 & -0.513314 & -1.558999 \\
\hline 4 & 1 & 0 & 0.889087 & -0.513314 & -1.558999 \\
\hline 5 & 6 & 0 & 0.000000 & 0.000000 & 0.274459 \\
\hline 6 & 7 & 0 & 0.000000 & 0.000000 & 1.436874 \\
\hline
\end{tabular}

E-2(singlet)opt $=\mathbf{- 2 0 2 4 . 1 5 3 9 5 0 7}$ hartree

\begin{tabular}{|c|c|c|c|c|c|}
\hline \multirow{2}{*}{$\begin{array}{l}\text { Center } \\
\text { Number }\end{array}$} & \multirow{2}{*}{$\begin{array}{l}\text { Atomic } \\
\text { Number }\end{array}$} & \multirow{2}{*}{$\begin{array}{l}\text { Atomic } \\
\text { Type }\end{array}$} & \multicolumn{3}{|c|}{ Coordinates (Angstroms) } \\
\hline & & & $\mathrm{X}$ & $\mathrm{Y}$ & Z \\
\hline 1 & 42 & 0 & -1.361712 & -0.046259 & -0.515761 \\
\hline 2 & 15 & 0 & 0.977850 & 0.056694 & -0.049743 \\
\hline 3 & 16 & 0 & -0.787848 & -2.353662 & -0.648646 \\
\hline
\end{tabular}




\begin{tabular}{|c|c|c|c|c|c|}
\hline 4 & 7 & 0 & -1.901331 & 0.472075 & -2.376480 \\
\hline 5 & 6 & 0 & 0.915115 & -2.428446 & -1.119969 \\
\hline 6 & 6 & 0 & -0.635770 & 0.442005 & -2.366461 \\
\hline 7 & 6 & 0 & -3.681553 & 0.295379 & 0.001760 \\
\hline 8 & 6 & 0 & 1.760001 & -1.309197 & -0.970503 \\
\hline 9 & 6 & 0 & -2.975625 & 1.515764 & 0.231171 \\
\hline 10 & 6 & 0 & -2.149292 & -0.065081 & 1.695289 \\
\hline 11 & 6 & 0 & 1.922933 & 1.582910 & -0.417510 \\
\hline 12 & 6 & 0 & -2.018719 & 1.306603 & 1.272061 \\
\hline 13 & 6 & 0 & 1.574121 & -0.292842 & 1.654452 \\
\hline 14 & 6 & 0 & 1.267307 & 2.690693 & -0.967575 \\
\hline 15 & 1 & 0 & 0.196729 & 2.614545 & -1.186644 \\
\hline 16 & 6 & 0 & 1.882026 & 0.739543 & 2.553645 \\
\hline 17 & 1 & 0 & 1.854467 & 1.780124 & 2.219729 \\
\hline 18 & 6 & 0 & -1.500433 & -0.713721 & 2.870238 \\
\hline 19 & 1 & 0 & -1.121597 & -1.719311 & 2.631992 \\
\hline 20 & 1 & 0 & -2.220669 & -0.830822 & 3.697132 \\
\hline 21 & 1 & 0 & -0.655864 & -0.132512 & 3.261956 \\
\hline 22 & 6 & 0 & -3.167821 & -0.685096 & 0.899902 \\
\hline 23 & 6 & 0 & 1.640869 & -1.617057 & 2.112128 \\
\hline 24 & 1 & 0 & 1.407033 & -2.440123 & 1.432162 \\
\hline 25 & 6 & 0 & 3.288286 & 1.699149 & -0.109633 \\
\hline 26 & 1 & 0 & 3.809227 & 0.867269 & 0.372433 \\
\hline 27 & 6 & 0 & 3.981344 & 2.877025 & -0.377963 \\
\hline 28 & 1 & 0 & 5.044140 & 2.948393 & -0.135024 \\
\hline 29 & 6 & 0 & 1.423624 & -3.590089 & -1.722417 \\
\hline 30 & 1 & 0 & 0.772387 & -4.461353 & -1.829122 \\
\hline 31 & 6 & 0 & 3.066870 & -1.352040 & -1.462460 \\
\hline 32 & 1 & 0 & 3.716553 & -0.478134 & -1.369903 \\
\hline 33 & 6 & 0 & 3.317412 & 3.966822 & -0.943066 \\
\hline 34 & 1 & 0 & 3.860296 & 4.892338 & -1.148680 \\
\hline 35 & 6 & 0 & 1.997065 & -1.898201 & 3.429426 \\
\hline 36 & 1 & 0 & 2.045504 & -2.937024 & 3.764437 \\
\hline 37 & 6 & 0 & 0.378787 & 0.551574 & -3.435615 \\
\hline 38 & 1 & 0 & 1.330699 & 0.972120 & -3.085966 \\
\hline 39 & 1 & 0 & 0.011084 & 1.129761 & -4.295086 \\
\hline 40 & 1 & 0 & 0.601856 & -0.468382 & -3.786221 \\
\hline 41 & 6 & 0 & 3.548889 & -2.495053 & -2.099576 \\
\hline 42 & 1 & 0 & 4.563510 & -2.509872 & -2.502946 \\
\hline 43 & 6 & 0 & 1.956992 & 3.873763 & -1.231028 \\
\hline 44 & 1 & 0 & 1.427031 & 4.727250 & -1.660393 \\
\hline 45 & 6 & 0 & 2.235584 & 0.458074 & 3.872356 \\
\hline 46 & 1 & 0 & 2.475513 & 1.277336 & 4.554587 \\
\hline 47 & 6 & 0 & 2.290866 & -0.862830 & 4.316113 \\
\hline
\end{tabular}




$\begin{array}{rrrrrr}48 & 1 & 0 & 2.570454 & -1.084742 & 5.348585 \\ 49 & 6 & 0 & 2.725472 & -3.617172 & -2.213464 \\ 50 & 1 & 0 & 3.097750 & -4.520679 & -2.703166 \\ 51 & 6 & 0 & -4.750864 & 0.110543 & -1.013408 \\ 52 & 1 & 0 & -4.461101 & 0.552569 & -1.977709 \\ 53 & 1 & 0 & -5.690785 & 0.590733 & -0.697244 \\ 54 & 1 & 0 & -4.967054 & -0.950637 & -1.190804 \\ 55 & 6 & 0 & -3.667650 & -2.075190 & 1.080848 \\ 56 & 1 & 0 & -4.104016 & -2.481926 & 0.158964 \\ 57 & 1 & 0 & -4.445954 & -2.119101 & 1.860271 \\ 58 & 1 & 0 & -2.863685 & -2.758505 & 1.387489 \\ 59 & 6 & 0 & -1.231926 & 2.370231 & 1.957513 \\ 60 & 1 & 0 & -0.407661 & 1.947263 & 2.545119 \\ 61 & 1 & 0 & -1.862912 & 2.940825 & 2.659387 \\ 62 & 1 & 0 & -0.796495 & 3.094759 & 1.254485 \\ 63 & 6 & 0 & -3.211017 & 2.788314 & -0.505168 \\ 64 & 1 & 0 & -2.359065 & 3.476878 & -0.420624 \\ 65 & 1 & 0 & -4.092590 & 3.318540 & -0.109248 \\ 66 & 1 & 0 & -3.393109 & 2.610700 & -1.574657\end{array}$

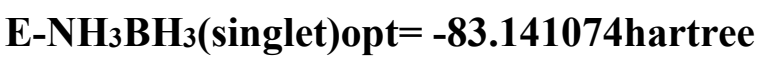

\begin{tabular}{|c|c|c|c|c|c|}
\hline \multirow{2}{*}{$\begin{array}{l}\text { Center } \\
\text { Number }\end{array}$} & \multirow{2}{*}{$\begin{array}{l}\text { Atomic } \\
\text { Number }\end{array}$} & \multirow{2}{*}{$\begin{array}{c}\text { Atomic } \\
\text { Type }\end{array}$} & \multicolumn{3}{|c|}{ Coordinates (Angstroms) } \\
\hline & & & $\mathrm{X}$ & $\mathrm{Y}$ & $Z$ \\
\hline 1 & 7 & 0 & -0.000000 & 0.000000 & 0.719807 \\
\hline 2 & 1 & 0 & 0.000000 & 0.946053 & 1.100589 \\
\hline 3 & 1 & 0 & 0.819306 & -0.473027 & 1.100589 \\
\hline 4 & 1 & 0 & -0.819306 & -0.473027 & 1.100589 \\
\hline 5 & 5 & 0 & -0.000000 & 0.000000 & -0.925222 \\
\hline 6 & 1 & 0 & -1.019933 & 0.588858 & -1.238102 \\
\hline 7 & 1 & 0 & 1.019933 & 0.588858 & -1.238102 \\
\hline 8 & 1 & 0 & -0.000000 & -1.177717 & -1.238102 \\
\hline
\end{tabular}

\section{E-TS1 (singlet)opt $=\mathbf{- 2 1 0 7 . 2 7 1 3 6 4 9}$ hartree}

\begin{tabular}{cccccc}
$\begin{array}{l}\text { Center } \\
\text { Number }\end{array}$ & $\begin{array}{c}\text { Atomic } \\
\text { Number }\end{array}$ & $\begin{array}{c}\text { Atomic } \\
\text { Type }\end{array}$ & \multicolumn{2}{c}{ Coordinates (Angstroms) } \\
- & X & Y & Z \\
1 & 42 & 0 & -1.092087 & -0.503614 & 0.314267 \\
2 & 15 & 0 & 1.154412 & 0.274461 & 0.056460 \\
3 & 16 & 0 & -1.392203 & 1.220921 & -1.330772 \\
4 & 7 & 0 & -2.052233 & 0.154778 & 1.945907 \\
5 & 6 & 0 & -0.130530 & 2.417948 & -1.006772
\end{tabular}


6

10

11

12

13

14

15

16

17

18

19

20

21

22

23

24

25

26

27

28

29

30

31

32

33

34

35

36

37

38

39

40

41

42

43

44

45

46

47

48

49
$-0.982513$

$-2.862586$

1.046062

$-1.792722$

$-1.007181$

2.343474

$-0.630079$

2.179045

1.922293

0.879585

3.090942

3.271427

$-0.205421$

$-0.262653$

$-0.572799$

0.856851

$-2.375941$

1.986876

1.282544

3.690316

4.054697

4.578015

5.621565

$-0.332355$

$-1.254273$

1.992479

2.898838

4.138520

4.836183

2.681221

2.519365

$-0.467009$

0.628419

$-0.913292$

$-0.746680$

1.775197

2.508764

2.809881

2.461182

3.783257

4.492119

3.577631

4.121436

0.613337
0.825222

$-2.106235$

2.056773

$-2.662697$

$-2.270836$

0.100609

$-2.760151$

$-0.330644$

$-0.461772$

$-0.781936$

$-1.385170$

$-1.803878$

$-2.341544$

$-1.410664$

$-3.151470$

$-2.540979$

$-1.851712$

0.198192

1.020826

0.477498

0.873980

0.324240

0.625736

3.755097

4.029046

3.036367

2.767983

$-0.222419$

$-0.346411$

$-0.317471$

0.110211

2.001058

2.010567

2.094644

2.893193

4.368891

5.130676

$-0.620748$

$-1.061649$

$-1.902335$

$-2.721068$

$-1.372044$

$-1.775230$

4.721332
1.868889

0.147550

$-0.322702$

0.909470

$-1.227429$

1.441400

0.076208

$-1.340820$

2.652047

2.751698

$-1.181197$

$-0.187413$

$-2.481894$

$-3.065958$

$-3.133707$

$-2.292279$

$-1.168707$

$-2.625360$

$-2.775435$

1.312354

0.360482

2.374518

2.257121

$-1.377536$

$-1.895513$

$-0.009365$

0.538919

3.581099

4.412655

$-3.717928$

$-4.710165$

2.596710

2.674678

3.596788

2.013241

$-0.357119$

$-0.084736$

3.715884

4.652696

$-2.274759$

$-2.128676$

$-3.548355$

$-4.405748$

$-1.046635$ 


\begin{tabular}{|c|c|c|c|c|c|}
\hline 50 & 1 & 0 & 0.434302 & 5.764583 & -1.318653 \\
\hline 51 & 6 & 0 & -4.229989 & -1.824779 & 0.657307 \\
\hline 52 & 1 & 0 & -4.195187 & -1.344190 & 1.645700 \\
\hline 53 & 1 & 0 & -4.817580 & -2.751475 & 0.758787 \\
\hline 54 & 1 & 0 & -4.780425 & -1.152798 & -0.013350 \\
\hline 55 & 6 & 0 & -3.177524 & -1.333806 & -2.311482 \\
\hline 56 & 1 & 0 & -3.863718 & -0.532109 & -2.002991 \\
\hline 57 & 1 & 0 & -3.783371 & -2.134284 & -2.765863 \\
\hline 58 & 1 & 0 & -2.536498 & -0.921261 & -3.101309 \\
\hline 59 & 6 & 0 & 0.626988 & -3.483991 & 0.418257 \\
\hline 60 & 1 & 0 & 1.446054 & -3.211369 & -0.259376 \\
\hline 61 & 1 & 0 & 0.494836 & -4.575996 & 0.335470 \\
\hline 62 & 1 & 0 & 0.966785 & -3.277281 & 1.443227 \\
\hline 63 & 6 & 0 & -1.876697 & -3.065907 & 2.340500 \\
\hline 64 & 1 & 0 & -0.895058 & -3.039299 & 2.833748 \\
\hline 65 & 1 & 0 & -2.257419 & -4.095519 & 2.440516 \\
\hline 66 & 1 & 0 & -2.551325 & -2.410176 & 2.907562 \\
\hline 67 & 5 & 0 & -3.359669 & 2.289537 & -0.515444 \\
\hline 68 & 1 & 0 & -2.779201 & 2.725229 & 0.453501 \\
\hline 69 & 1 & 0 & -3.935754 & 1.227424 & -0.426160 \\
\hline 70 & 1 & 0 & -3.546520 & 2.993555 & -1.477758 \\
\hline 71 & 7 & 0 & -5.390094 & 3.177875 & 0.674827 \\
\hline 72 & 1 & 0 & -5.371889 & 4.119997 & 1.060359 \\
\hline 73 & 1 & 0 & -5.234835 & 2.547621 & 1.459560 \\
\hline 74 & 1 & 0 & -6.345024 & 3.013827 & 0.361890 \\
\hline
\end{tabular}

E-Int1(singlet)opt $=\mathbf{- 2 0 5 0 . 7 7 6 3 6 6}$ hartree

\begin{tabular}{cccccc} 
Center & Atomic & Atomic & \multicolumn{3}{c}{ Coordinates (Angstroms) } \\
Number & Number & Type & X & Y & $Z$ \\
-------1.304704 & 0.025143 & -0.441983 \\
1 & 42 & 0 & -0.626750 & -2.126721 & -1.365798 \\
2 & 16 & 0 & 1.035239 & 0.068607 & 0.030048 \\
3 & 15 & 0 & 1.151046 & -2.194467 & -1.525748 \\
4 & 6 & 0 & 1.935384 & -1.214012 & -0.912857 \\
5 & 6 & 0 & 3.332217 & -1.311067 & -0.987765 \\
6 & 6 & 0 & 3.929942 & -2.348123 & -1.696880 \\
7 & 6 & 0 & 3.134924 & -3.316984 & -2.317154 \\
8 & 6 & 0 & 1.747875 & -3.251217 & -2.220422 \\
9 & 6 & 0 & 1.914328 & 1.627615 & -0.322200 \\
10 & 6 & 0 & 1.659178 & 2.744141 & 0.492932 \\
11 & 6 & 0 & 2.177267 & 3.998467 & 0.180355 \\
12 & 6 & 0 & 2.944252 & 4.171338 & -0.973923
\end{tabular}


14

15

16

17

18

19

20

21

22

23

24

25

26

27

28

29

30

31

32

33

34

35

36

37

38

39

40

41

42

43

44

45

46

47

48

49

50

51

52

53

54

55

56

57
3.165968

2.651260

1.265979

1.643709

2.418894

2.773175

2.376979

1.629168

$-2.443564$

$-3.293126$

$-3.470242$

$-2.718816$

$-2.100373$

$-4.022241$

$-4.371851$

$-2.666073$

$-1.349385$

$-2.159582$

3.953767

5.018854

3.601475

1.119711

1.031234

1.979332

3.359170

3.748149

2.818018

0.672400

2.766887

3.373481

2.658426

1.326918

$-4.239941$

$-3.437859$

$-4.983727$

$-3.975917$

$-4.506898$

$-5.374758$

$-1.667743$

$-2.928967$

$-3.374516$

$-0.710365$

$-0.700855$

$-2.043357$
3.082382

1.825397

$-1.686759$

$-0.402580$

0.379411

$-0.095873$

$-1.365482$

$-2.167872$

$-1.066580$

$-0.866497$

0.555634

1.204661

0.211421

$-1.940936$

1.223861

2.671824

0.484331

$-2.393430$

$-0.568741$

$-2.412347$

$-4.134044$

$-4.013892$

2.626850

4.847306

5.152394

3.208904

0.988453

$-2.315701$

1.366910

0.532396

$-1.735108$

$-3.173135$

$-1.655011$

$-2.871180$

$-2.184632$

2.196776

0.614497

1.409270

2.999891

3.256839

2.962939

$-0.355294$

1.368273

0.680438
$-1.817200$

$-1.498013$

2.132986

1.709269

2.572457

3.837699

4.251336

3.386470

1.266994

0.113846

$-0.050146$

0.991907

1.802411

$-0.619469$

$-1.031197$

1.250545

3.059220

1.884674

$-0.476663$

$-1.757307$

$-2.872641$

$-2.687118$

1.382773

0.840416

$-1.218265$

$-2.733499$

$-2.181084$

1.460125

2.261260

4.500663

5.240122

3.691857

$-1.657139$

$-0.662105$

$-0.136199$

$-1.356670$

$-1.935038$

$-0.610533$

1.576392

0.358752

2.043650

3.360461

2.971517

3.893855 


\begin{tabular}{rrrrrr}
\hline 58 & 1 & 0 & -1.824902 & -3.137049 & 1.145666 \\
59 & 1 & 0 & -1.391686 & -2.329547 & 2.666569 \\
60 & 1 & 0 & -3.067074 & -2.804193 & 2.357862 \\
61 & 5 & 0 & -1.096118 & -1.431064 & -3.083608 \\
62 & 1 & 0 & -0.111898 & -1.295058 & -3.776249 \\
63 & 1 & 0 & -2.000608 & -2.102408 & -3.540518 \\
64 & 1 & 0 & -1.655928 & -0.294701 & -2.894119 \\
65 & 6 & 0 & -0.790132 & 1.900684 & -1.216963 \\
66 & 7 & 0 & -0.396288 & 1.043744 & -2.022034 \\
67 & 6 & 0 & -0.747145 & 3.448092 & -1.138349 \\
68 & 1 & 0 & -0.493594 & 3.778280 & -0.120257 \\
69 & 1 & 0 & 0.017873 & 3.850364 & -1.819874 \\
70 & 1 & 0 & -1.717608 & 3.888492 & -1.407917 \\
-------------------- & &
\end{tabular}

\section{E-Int2(singlet)opt $=\mathbf{- 2 0 5 0 . 7 8 3 3 6 1 \text { hartree }}$}

\begin{tabular}{rrrrrr}
$\begin{array}{l}\text { Center } \\
\text { Number }\end{array}$ & $\begin{array}{c}\text { Atomic } \\
\text { Number }\end{array}$ & $\begin{array}{r}\text { Atomic } \\
\text { Type }\end{array}$ & \multicolumn{2}{c}{ Coordinates (Angstroms) } \\
-1 & 42 & 0 & 1.297028 & -0.034225 & 0.422491 \\
2 & 16 & 0 & 0.510722 & -1.857780 & 1.836685 \\
3 & 15 & 0 & -1.045397 & 0.043421 & -0.032536 \\
4 & 6 & 0 & -1.264240 & -1.773253 & 2.018615 \\
5 & 6 & 0 & -2.000993 & -0.919006 & 1.194254 \\
6 & 6 & 0 & -3.399276 & -0.907135 & 1.298346 \\
7 & 6 & 0 & -4.043202 & -1.706166 & 2.237768 \\
8 & 6 & 0 & -3.294943 & -2.548094 & 3.066472 \\
9 & 6 & 0 & -1.908750 & -2.594861 & 2.948703 \\
10 & 6 & 0 & -1.822043 & 1.693755 & -0.056993 \\
11 & 6 & 0 & -1.517443 & 2.566247 & -1.116308 \\
12 & 6 & 0 & -1.952886 & 3.889043 & -1.108768 \\
13 & 6 & 0 & -2.684036 & 4.379258 & -0.024510 \\
14 & 6 & 0 & -2.953034 & 3.538067 & 1.055368 \\
15 & 6 & 0 & -2.521262 & 2.211337 & 1.042063 \\
16 & 6 & 0 & -1.424418 & -2.143158 & -1.654915 \\
17 & 6 & 0 & -1.715690 & -0.773878 & -1.547385 \\
18 & 6 & 0 & -2.460386 & -0.172051 & -2.567556 \\
19 & 6 & 0 & -2.868661 & -0.911004 & -3.680887 \\
20 & 6 & 0 & -2.557877 & -2.264932 & -3.782211 \\
21 & 6 & 0 & -1.841688 & -2.884406 & -2.755386 \\
22 & 6 & 0 & 2.332771 & -1.570356 & -0.983569 \\
23 & 6 & 0 & 3.216524 & -1.156047 & 0.083609 \\
24 & 6 & 0 & 3.481857 & 0.250229 & -0.097241
\end{tabular}


25

26

27

28

29

30

31

32

33

34

35

36

37

38

39

40

41

42

43

44

45

46

47

48

49

50

51

52

53

54

55

56

57

58

59

60

61

62

63

64

65

66

67

68
2.749200

2.055705

3.895034

4.442021

2.779068

1.296505

1.957084

$-3.985719$

$-5.132518$

$-3.797969$

$-1.317847$

$-0.916242$

$-1.718201$

$-3.034296$

$-3.507463$

$-2.723643$

$-0.855744$

$-2.742083$

$-3.443883$

$-2.881403$

$-1.606585$

4.150942

3.256998

4.829969

4.111901

4.559047

5.445291

1.795635

3.094966

3.487072

0.602237

0.704038

1.983516

1.593868

1.178341

2.828336

1.196659

0.307620

2.144814

1.738900

0.983385

1.052536

0.875497

1.247175
0.679175

$-0.437127$

$-2.069297$

1.074142

2.043193

$-0.423807$

$-2.985324$

$-0.269799$

$-1.685344$

$-3.178659$

$-3.262890$

2.201509

4.542517

5.414447

3.915152

1.572893

$-2.630393$

0.881043

$-0.421647$

$-2.840382$

$-3.950452$

$-1.559342$

$-2.923986$

$-2.481013$

2.119517

0.689701

1.090624

2.346776

2.805548

2.092232

$-1.268912$

0.494774

$-0.475815$

$-3.509420$

$-3.060714$

$-3.553176$

$-0.468874$

0.042851

$-0.905245$

0.404105

3.174047

2.016142

4.598750

5.126010
$-1.259553$

$-1.806225$

1.047651

0.690883

$-1.860224$

$-3.087423$

$-1.265404$

0.628655

2.318160

3.803476

3.580081

$-1.956195$

$-1.953328$

$-0.018688$

1.918480

1.905950

$-0.855003$

$-2.498737$

$-4.470988$

$-4.652683$

$-2.814058$

1.985928

1.314282

0.630782

0.777324

1.713136

0.232261

$-2.249023$

$-1.135191$

$-2.704013$

$-3.176032$

$-3.209194$

$-3.948807$

$-0.368459$

$-2.035457$

$-1.632333$

2.957197

3.601157

3.580217

2.192673

0.873762

0.731601

1.044749

0.153865 


\begin{tabular}{llllll}
\hline 69 & 1 & 0 & -0.174557 & 4.893560 & 1.193162 \\
70 & 1 & 0 & 1.457726 & 4.941861 & 1.911756
\end{tabular}

\section{E-TS2'(singlet)opt $=\mathbf{- 2 0 5 0 . 7 1 6 2 7 7 7}$ hartree}

\begin{tabular}{|c|c|c|c|c|c|}
\hline \multirow{2}{*}{$\begin{array}{l}\text { Center } \\
\text { Number }\end{array}$} & \multirow{2}{*}{$\begin{array}{l}\text { Atomic } \\
\text { Number }\end{array}$} & \multirow{2}{*}{$\begin{array}{l}\text { Atomic } \\
\text { Type }\end{array}$} & \multicolumn{3}{|c|}{ Coordinates (Angstroms) } \\
\hline & & & $\mathrm{X}$ & $\mathrm{Y}$ & Z \\
\hline 1 & 42 & 0 & -1.337534 & 0.170081 & -0.346692 \\
\hline 2 & 16 & 0 & -0.613146 & -1.678462 & -1.677646 \\
\hline 3 & 15 & 0 & 1.059428 & 0.100089 & 0.087749 \\
\hline 4 & 6 & 0 & 1.106211 & -2.157169 & -1.541208 \\
\hline 5 & 6 & 0 & 1.876038 & -1.324461 & -0.727252 \\
\hline 6 & 6 & 0 & 3.226931 & -1.640471 & -0.523038 \\
\hline 7 & 6 & 0 & 3.793159 & -2.755192 & -1.132713 \\
\hline 8 & 6 & 0 & 3.007342 & -3.576464 & -1.945867 \\
\hline 9 & 6 & 0 & 1.661528 & -3.283822 & -2.151635 \\
\hline 10 & 6 & 0 & 2.034867 & 1.537412 & -0.483743 \\
\hline 11 & 6 & 0 & 1.722342 & 2.800303 & 0.047966 \\
\hline 12 & 6 & 0 & 2.343586 & 3.950784 & -0.430181 \\
\hline 13 & 6 & 0 & 3.268020 & 3.867550 & -1.471411 \\
\hline 14 & 6 & 0 & 3.561514 & 2.625614 & -2.032838 \\
\hline 15 & 6 & 0 & 2.947837 & 1.471561 & -1.546234 \\
\hline 16 & 6 & 0 & 1.527543 & -1.488138 & 2.311975 \\
\hline 17 & 6 & 0 & 1.670736 & -0.189732 & 1.796365 \\
\hline 18 & 6 & 0 & 2.201564 & 0.801557 & 2.629332 \\
\hline 19 & 6 & 0 & 2.557092 & 0.508335 & 3.946921 \\
\hline 20 & 6 & 0 & 2.398063 & -0.781069 & 4.450636 \\
\hline 21 & 6 & 0 & 1.888141 & -1.783393 & 3.623689 \\
\hline 22 & 6 & 0 & -2.321826 & -1.074863 & 1.310902 \\
\hline 23 & 6 & 0 & -3.243956 & -0.879641 & 0.214705 \\
\hline 24 & 6 & 0 & -3.558760 & 0.525081 & 0.140116 \\
\hline 25 & 6 & 0 & -2.840212 & 1.182178 & 1.183683 \\
\hline 26 & 6 & 0 & -2.075366 & 0.214015 & 1.895915 \\
\hline 27 & 6 & 0 & -3.937377 & -1.969583 & -0.528835 \\
\hline 28 & 6 & 0 & -4.532072 & 1.177202 & -0.780773 \\
\hline 29 & 6 & 0 & -2.902243 & 2.631608 & 1.506754 \\
\hline 30 & 6 & 0 & -1.306136 & 0.499976 & 3.139176 \\
\hline 31 & 6 & 0 & -1.923541 & -2.399139 & 1.870539 \\
\hline 32 & 1 & 0 & 3.833799 & -1.000576 & 0.125039 \\
\hline 33 & 1 & 0 & 4.846524 & -2.992426 & -0.968243 \\
\hline 34 & 1 & 0 & 3.443718 & -4.460111 & -2.417178 \\
\hline 35 & 1 & 0 & 1.044852 & -3.932793 & -2.774484 \\
\hline 36 & 1 & 0 & 0.961559 & 2.882761 & 0.828229 \\
\hline
\end{tabular}




\begin{tabular}{|c|c|c|c|c|c|}
\hline 37 & 1 & 0 & 2.087617 & 4.921434 & 0.000995 \\
\hline 38 & 1 & 0 & 3.749830 & 4.771142 & -1.852362 \\
\hline 39 & 1 & 0 & 4.275579 & 2.550468 & -2.856652 \\
\hline 40 & 1 & 0 & 3.182984 & 0.509645 & -2.008521 \\
\hline 41 & 1 & 0 & 1.128294 & -2.280333 & 1.670690 \\
\hline 42 & 1 & 0 & 2.351378 & 1.815163 & 2.251753 \\
\hline 43 & 1 & 0 & 2.968759 & 1.296985 & 4.581425 \\
\hline 44 & 1 & 0 & 2.679504 & -1.008154 & 5.481441 \\
\hline 45 & 1 & 0 & 1.772330 & -2.802279 & 4.001975 \\
\hline 46 & 1 & 0 & -4.247949 & -1.655059 & -1.534401 \\
\hline 47 & 1 & 0 & -3.302670 & -2.858743 & -0.649309 \\
\hline 48 & 1 & 0 & -4.846136 & -2.296492 & 0.004803 \\
\hline 49 & 1 & 0 & -4.164779 & 2.151870 & -1.133718 \\
\hline 50 & 1 & 0 & -4.727238 & 0.561481 & -1.668867 \\
\hline 51 & 1 & 0 & -5.502799 & 1.359306 & -0.290144 \\
\hline 52 & 1 & 0 & -1.947950 & 3.001619 & 1.904002 \\
\hline 53 & 1 & 0 & -3.135950 & 3.239770 & 0.624416 \\
\hline 54 & 1 & 0 & -3.676868 & 2.827876 & 2.266444 \\
\hline 55 & 1 & 0 & -0.622583 & -0.314385 & 3.409216 \\
\hline 56 & 1 & 0 & -0.704878 & 1.417088 & 3.056146 \\
\hline 57 & 1 & 0 & -1.989437 & 0.644456 & 3.992553 \\
\hline 58 & 1 & 0 & -1.616559 & -3.110522 & 1.089167 \\
\hline 59 & 1 & 0 & -1.088322 & -2.308135 & 2.577712 \\
\hline 60 & 1 & 0 & -2.757485 & -2.870542 & 2.418769 \\
\hline 61 & 5 & 0 & -1.373044 & -2.567077 & -2.987237 \\
\hline 62 & 1 & 0 & -1.846501 & 0.548836 & -1.976461 \\
\hline 63 & 1 & 0 & -0.766920 & -3.381915 & -3.618099 \\
\hline 64 & 1 & 0 & -2.522865 & -2.298111 & -3.179826 \\
\hline 65 & 6 & 0 & -0.931215 & 1.741521 & -1.862759 \\
\hline 66 & 7 & 0 & -1.009423 & 2.322580 & -0.782016 \\
\hline 67 & 6 & 0 & -0.499109 & 2.030396 & -3.247521 \\
\hline 68 & 1 & 0 & -0.050918 & 3.030594 & -3.310089 \\
\hline 69 & 1 & 0 & 0.239634 & 1.286115 & -3.578415 \\
\hline 70 & 1 & 0 & -1.345200 & 1.970230 & -3.946529 \\
\hline
\end{tabular}

\section{E-Int3(singlet)opt $=\mathbf{- 2 0 5 0 . 7 7 0 8 4 8 7}$ hartree}

\begin{tabular}{|c|c|c|c|c|c|}
\hline \multirow{2}{*}{$\begin{array}{l}\text { Center } \\
\text { Number }\end{array}$} & \multirow{2}{*}{$\begin{array}{l}\text { Atomic } \\
\text { Number }\end{array}$} & \multirow{2}{*}{$\begin{array}{l}\text { Atomic } \\
\text { Type }\end{array}$} & \multicolumn{3}{|c|}{ Coordinates (Angstroms) } \\
\hline & & & $\mathrm{X}$ & $\mathrm{Y}$ & Z \\
\hline 1 & 42 & 0 & 1.266424 & -0.276982 & 0.157389 \\
\hline 2 & 16 & 0 & 0.704092 & -1.064449 & -2.212566 \\
\hline 3 & 15 & 0 & -1.114130 & 0.088399 & 0.075500 \\
\hline 4 & 6 & 0 & -1.048302 & -1.183598 & -2.375725 \\
\hline & & & 50 & & \\
\hline
\end{tabular}


6

6
7

8

9

10

11

12

13

14

15

16

17

18

19

20

21

22

23

24

25

26

27

28

29

30

31

32

33

34

35

36

37

38

39

40

41

42

43

44

45

46

47

48
$-1.893332$

$-3.276791$

$-3.821964$

$-2.978405$

$-1.599600$

$-2.069591$

$-1.853941$

$-2.462142$

$-3.286903$

$-3.494059$

$-2.893281$

$-1.310736$

$-1.717744$

$-2.491897$

$-2.823869$

$-2.407276$

$-1.657590$

2.549875

3.352304

3.518131

2.753542

2.152151

4.045544

4.336101

2.706505

1.353061

2.265833

$-3.930727$

$-4.902533$

$-3.399433$

$-0.935973$

$-1.185694$

$-2.283458$

$-3.759183$

$-4.126546$

$-3.052935$

$-0.708315$

$-2.853773$

$-3.422443$

$-2.672017$

$-1.339510$

4.203382

3.476120

5.034270
$-0.624007$

$-0.597173$

$-1.181285$

$-1.781291$

$-1.769792$

$-0.597110$

$-0.076814$

$-0.645160$

$-1.761933$

$-2.300475$

$-1.723258$

2.494679

1.820465

2.510923

3.852828

4.512926

3.823425

1.553878

0.358792

$-0.006416$

0.905101

1.866665

$-0.289604$

$-1.146961$

0.965084

3.043344

2.329264

$-0.122750$

$-1.170281$

$-2.248501$

$-2.208137$

0.779447

$-0.218639$

$-2.213883$

$-3.182693$

$-2.176238$

1.959575

2.004955

4.380681

5.560671

4.327074

$-1.362021$

$-0.193793$

0.166171
$-1.407171$

$-1.629361$

$-2.768541$

$-3.707690$

$-3.524615$

1.476386

2.764034

3.879708

3.735381

2.467402

1.348138

$-1.234641$

$-0.071660$

0.866345

0.659789

$-0.493023$

$-1.450251$

$-0.686511$

$-0.623835$

0.755331

1.533160

0.640735

$-1.773809$

1.243818

3.021821

1.086846

$-1.924710$

$-0.890965$

$-2.925974$

$-4.601114$

$-4.273131$

2.897189

4.869618

4.610494

2.343106

0.367119

$-1.978076$

1.764236

1.406277

$-0.652674$

$-2.366739$

$-1.599862$

$-2.709039$

$-1.949356$ 


\begin{tabular}{rrrrrr}
\hline 49 & 1 & 0 & 4.181890 & -1.329558 & 2.315290 \\
50 & 1 & 0 & 4.080882 & -2.076726 & 0.710879 \\
51 & 1 & 0 & 5.411612 & -0.965924 & 1.090624 \\
52 & 1 & 0 & 1.788749 & 1.447202 & 3.383207 \\
53 & 1 & 0 & 2.748617 & -0.033743 & 3.475727 \\
54 & 1 & 0 & 3.556622 & 1.543108 & 3.419943 \\
55 & 1 & 0 & 0.789806 & 3.502236 & 0.264256 \\
56 & 1 & 0 & 0.622751 & 2.776549 & 1.865293 \\
57 & 1 & 0 & 2.002200 & 3.825056 & 1.514380 \\
58 & 1 & 0 & 1.952207 & 1.678718 & -2.755451 \\
59 & 1 & 0 & 1.477438 & 3.077566 & -1.769165 \\
60 & 1 & 0 & 3.163119 & 2.870861 & -2.267195 \\
61 & 5 & 0 & 1.484426 & -2.881614 & -1.531076 \\
62 & 1 & 0 & 0.532677 & -0.004562 & 1.667535 \\
63 & 1 & 0 & 0.641896 & -3.751234 & -1.664823 \\
64 & 1 & 0 & 2.483314 & -2.994683 & -2.207401 \\
65 & 6 & 0 & 0.938839 & -2.140504 & 0.865384 \\
66 & 7 & 0 & 1.718206 & -2.381129 & -0.124937 \\
67 & 6 & 0 & 0.270798 & -3.077465 & 1.790575 \\
68 & 1 & 0 & -0.284987 & -2.569128 & 2.588027 \\
69 & 1 & 0 & -0.426102 & -3.716123 & 1.223275 \\
70 & 1 & 0 & 1.011611 & -3.754017 & 2.242802 \\
--------------------------------------------------- & & \\
\hline-51 & 1 & & &
\end{tabular}

\section{E-TS2(singlet)opt $=\mathbf{- 2 0 5 0 . 7 6 3 9 4 4 6 h a r t r e e}$}

\begin{tabular}{rrrrrr} 
Center & Atomic & Atomic & \multicolumn{2}{c}{ Coordinates (Angstroms) } \\
Number & Number & Type & X & Y & Z \\
-1 & 42 & 0 & -1.313210 & 0.165579 & 0.327262 \\
2 & 16 & 0 & -0.839804 & 2.125145 & -1.178273 \\
3 & 15 & 0 & 1.076135 & -0.098095 & 0.011548 \\
4 & 6 & 0 & 0.903951 & 2.426306 & -1.141239 \\
5 & 6 & 0 & 1.789936 & 1.427483 & -0.699444 \\
6 & 6 & 0 & 3.168147 & 1.603909 & -0.888258 \\
7 & 6 & 0 & 3.670368 & 2.793876 & -1.407337 \\
8 & 6 & 0 & 2.787726 & 3.814787 & -1.769491 \\
9 & 6 & 0 & 1.414284 & 3.625937 & -1.659198 \\
10 & 6 & 0 & 2.052237 & -0.460204 & 1.518407 \\
11 & 6 & 0 & 1.669630 & -1.605348 & 2.237239 \\
12 & 6 & 0 & 2.274946 & -1.932862 & 3.446353 \\
13 & 6 & 0 & 3.263440 & -1.102738 & 3.978878 \\
14 & 6 & 0 & 3.638682 & 0.046949 & 3.287909 \\
15 & 6 & 0 & 3.043050 & 0.364149 & 2.065827 \\
16 & 6 & 0 & 1.364272 & -1.090667 & -2.541704
\end{tabular}


17

18

19

20

21

22

23

24

25

26

27

28

29

30

31

32

33

34

35

36

37

38

39

40

41

42

43

44

45

46

47

48

49

50

51

52

53

54

55

56

57

58

59

60
1.705792

2.438876

2.805176

2.454614

1.738125

$-2.460839$

$-3.332269$

$-3.369288$

$-2.500605$

$-1.954474$

$-4.203329$

$-4.220317$

$-2.322728$

$-1.091795$

$-2.249057$

3.855618

4.747405

3.173439

0.718707

0.865281

1.964040

3.731671

4.400845

3.338708

0.793274

2.733958

3.374996

2.744755

1.468857

$-4.510300$

$-3.696230$

$-5.120693$

$-3.839720$

$-4.253758$

$-5.258814$

$-1.331597$

$-2.441945$

$-3.065373$

$-0.551497$

$-0.340301$

$-1.700678$

$-2.077976$

$-1.390849$

$-3.131981$
$-1.322563$

$-2.469722$

$-3.375743$

$-3.140838$

$-1.988134$

$-0.945871$

$-0.105504$

$-0.645556$

$-1.788886$

$-1.969805$

0.979794

$-0.144516$

$-2.730007$

$-3.114800$

$-0.815303$

0.795971

2.925654

4.755993

4.400715

$-2.235784$

$-2.832071$

$-1.348346$

0.710837

1.283517

$-0.192637$

$-2.663659$

$-4.269458$

$-3.849975$

$-1.788921$

1.678306

1.574686

0.568717

$-0.470495$

0.954091

$-0.504811$

$-3.203786$

$-2.231590$

$-3.544232$

$-2.928427$

$-3.362917$

$-4.021350$

0.229246

$-1.406569$

$-1.167444$
$-1.199531$

$-0.879531$

$-1.877508$

$-3.204885$

$-3.536534$

$-1.402752$

$-0.614252$

0.726671

0.754539

$-0.552337$

$-1.148185$

1.840973

1.896090

$-0.955515$

$-2.870531$

$-0.620125$

$-1.530973$

$-2.168581$

$-1.989836$

1.843357

3.983497

4.934722

3.703004

1.556538

$-2.802926$

0.154810

$-1.611834$

$-3.983506$

$-4.576843$

$-0.359640$

$-1.921555$

$-1.602876$

2.818344

1.860946

1.756628

1.891124

2.866787

1.853619

$-1.892151$

$-0.191721$

$-1.105989$

$-3.170867$

$-3.217029$

$-3.429557$ 


\begin{tabular}{rrrrrr}
\hline 61 & 5 & 0 & -1.804323 & 3.197462 & 0.332458 \\
62 & 1 & 0 & -0.937022 & -0.209193 & 1.972946 \\
63 & 1 & 0 & -1.113705 & 4.155049 & 0.632835 \\
64 & 1 & 0 & -2.875071 & 3.441362 & -0.173654 \\
65 & 6 & 0 & -0.790111 & 1.497444 & 1.830063 \\
66 & 7 & 0 & -1.821014 & 2.064891 & 1.346382 \\
67 & 6 & 0 & 0.117785 & 1.935720 & 2.909020 \\
68 & 1 & 0 & 0.623515 & 1.101785 & 3.409653 \\
69 & 1 & 0 & 0.894565 & 2.568952 & 2.449828 \\
70 & 1 & 0 & -0.411086 & 2.556306 & 3.645804
\end{tabular}

\section{E-Int4(triplet)opt $=\mathbf{- 2 0 5 0 . 7 8 9 8 9 1 7}$ hartree}

\begin{tabular}{|c|c|c|c|c|c|}
\hline \multirow{2}{*}{$\begin{array}{l}\text { Center } \\
\text { Number }\end{array}$} & \multirow{2}{*}{$\begin{array}{l}\text { Atomic } \\
\text { Number }\end{array}$} & \multirow{2}{*}{$\begin{array}{l}\text { Atomic } \\
\text { Type }\end{array}$} & \multicolumn{3}{|c|}{ Coordinates (Angstroms) } \\
\hline & & & $\mathrm{X}$ & Y & $Z$ \\
\hline 1 & 42 & 0 & 1.317054 & 0.254759 & -0.148714 \\
\hline 2 & 16 & 0 & 0.651471 & 2.211697 & 1.200536 \\
\hline 3 & 15 & 0 & -1.150366 & -0.057227 & -0.090772 \\
\hline 4 & 6 & 0 & -1.071797 & 2.450378 & 1.028909 \\
\hline 5 & 6 & 0 & -1.922891 & 1.480221 & 0.452428 \\
\hline 6 & 6 & 0 & -3.312839 & 1.666860 & 0.456321 \\
\hline 7 & 6 & 0 & -3.873534 & 2.838417 & 0.951653 \\
\hline 8 & 6 & 0 & -3.031117 & 3.824507 & 1.477406 \\
\hline 9 & 6 & 0 & -1.657362 & 3.631299 & 1.527958 \\
\hline 10 & 6 & 0 & -1.848565 & -0.585321 & -1.685158 \\
\hline 11 & 6 & 0 & -1.300316 & -1.746525 & -2.253964 \\
\hline 12 & 6 & 0 & -1.711687 & -2.196515 & -3.504292 \\
\hline 13 & 6 & 0 & -2.670833 & -1.478686 & -4.220895 \\
\hline 14 & 6 & 0 & -3.210348 & -0.316572 & -3.675168 \\
\hline 15 & 6 & 0 & -2.806273 & 0.127323 & -2.415069 \\
\hline 16 & 6 & 0 & -1.674450 & -0.926442 & 2.465164 \\
\hline 17 & 6 & 0 & -1.871596 & -1.234190 & 1.109366 \\
\hline 18 & 6 & 0 & -2.550390 & -2.410329 & 0.773188 \\
\hline 19 & 6 & 0 & -3.008820 & -3.269802 & 1.773417 \\
\hline 20 & 6 & 0 & -2.798188 & -2.962864 & 3.115941 \\
\hline 21 & 6 & 0 & -2.133657 & -1.783214 & 3.460254 \\
\hline 22 & 6 & 0 & 3.289913 & -0.125516 & 1.079592 \\
\hline 23 & 6 & 0 & 3.523535 & -0.640879 & -0.230795 \\
\hline 24 & 6 & 0 & 2.586503 & -1.690176 & -0.475101 \\
\hline 25 & 6 & 0 & 1.810925 & -1.872073 & 0.722012 \\
\hline 26 & 6 & 0 & 2.243332 & -0.905045 & 1.683663 \\
\hline 27 & 6 & 0 & 4.614169 & -0.257187 & -1.163397 \\
\hline 28 & 6 & 0 & 2.548940 & -2.534215 & -1.703838 \\
\hline
\end{tabular}




\begin{tabular}{|c|c|c|c|c|c|}
\hline 29 & 6 & 0 & 0.889940 & -3.016621 & 0.975251 \\
\hline 30 & 6 & 0 & 1.807204 & -0.783951 & 3.102935 \\
\hline 31 & 6 & 0 & 4.051783 & 0.960452 & 1.758102 \\
\hline 32 & 1 & 0 & -3.964066 & 0.871251 & 0.081608 \\
\hline 33 & 1 & 0 & -4.955900 & 2.979881 & 0.939368 \\
\hline 34 & 1 & 0 & -3.456261 & 4.752668 & 1.867818 \\
\hline 35 & 1 & 0 & -1.008922 & 4.394110 & 1.964794 \\
\hline 36 & 1 & 0 & -0.524804 & -2.295079 & -1.708898 \\
\hline 37 & 1 & 0 & -1.272614 & -3.103258 & -3.926905 \\
\hline 38 & 1 & 0 & -2.988943 & -1.821765 & -5.207809 \\
\hline 39 & 1 & 0 & -3.952079 & 0.257785 & -4.234719 \\
\hline 40 & 1 & 0 & -3.230620 & 1.049138 & -2.012470 \\
\hline 41 & 1 & 0 & -1.149570 & -0.003111 & 2.735267 \\
\hline 42 & 1 & 0 & -2.729629 & -2.662405 & -0.275121 \\
\hline 43 & 1 & 0 & -3.539281 & -4.184367 & 1.497710 \\
\hline 44 & 1 & 0 & -3.158258 & -3.637272 & 3.896161 \\
\hline 45 & 1 & 0 & -1.974229 & -1.529371 & 4.511126 \\
\hline 46 & 1 & 0 & 4.247371 & -0.086795 & -2.185190 \\
\hline 47 & 1 & 0 & 5.134063 & 0.653358 & -0.846271 \\
\hline 48 & 1 & 0 & 5.361506 & -1.065483 & -1.215095 \\
\hline 49 & 1 & 0 & 1.655446 & -3.172122 & -1.739093 \\
\hline 50 & 1 & 0 & 2.559722 & -1.929537 & -2.623688 \\
\hline 51 & 1 & 0 & 3.421175 & -3.207180 & -1.759284 \\
\hline 52 & 1 & 0 & 0.246995 & -2.857171 & 1.849789 \\
\hline 53 & 1 & 0 & 0.228521 & -3.235105 & 0.124331 \\
\hline 54 & 1 & 0 & 1.471057 & -3.934008 & 1.163886 \\
\hline 55 & 1 & 0 & 1.567934 & 0.255878 & 3.372606 \\
\hline 56 & 1 & 0 & 0.917972 & -1.391835 & 3.313505 \\
\hline 57 & 1 & 0 & 2.601176 & -1.122238 & 3.788501 \\
\hline 58 & 1 & 0 & 4.447959 & 1.692543 & 1.042012 \\
\hline 59 & 1 & 0 & 3.422752 & 1.510590 & 2.471620 \\
\hline 60 & 1 & 0 & 4.906114 & 0.556794 & 2.326137 \\
\hline 61 & 5 & 0 & 2.950309 & 2.436687 & -1.901014 \\
\hline 62 & 1 & 0 & 0.495900 & 0.481938 & -2.785566 \\
\hline 63 & 1 & 0 & 2.818527 & 3.190527 & -2.840614 \\
\hline 64 & 6 & 0 & 0.680524 & 1.271601 & -2.031037 \\
\hline 65 & 7 & 0 & 1.998255 & 1.451258 & -1.682254 \\
\hline 66 & 6 & 0 & -0.182435 & 2.488822 & -2.208085 \\
\hline 67 & 1 & 0 & -1.256475 & 2.258025 & -2.246873 \\
\hline 68 & 1 & 0 & -0.012872 & 3.216459 & -1.402041 \\
\hline 69 & 1 & 0 & 0.070703 & 2.992419 & -3.156609 \\
\hline 70 & 1 & 0 & 3.917958 & 2.531330 & -1.180718 \\
\hline
\end{tabular}

\section{E-Int5(triplet)opt $=\mathbf{- 2 1 3 3 . 9 4 8 4 8 4 6 h a r t r e e}$}




\begin{tabular}{|c|c|c|c|c|c|}
\hline \multirow{2}{*}{$\begin{array}{l}\text { Center } \\
\text { Number }\end{array}$} & \multirow{2}{*}{$\begin{array}{l}\text { Atomic } \\
\text { Number }\end{array}$} & \multirow{2}{*}{$\begin{array}{l}\text { Atomic } \\
\text { Type }\end{array}$} & \multicolumn{3}{|c|}{ Coordinates (Angstroms) } \\
\hline & & & $\mathrm{X}$ & $\mathrm{Y}$ & $\mathrm{Z}$ \\
\hline 1 & 42 & 0 & 0.953735 & -0.622609 & 0.298630 \\
\hline 2 & 16 & 0 & -0.381497 & -1.654954 & 2.098620 \\
\hline 3 & 15 & 0 & -1.266697 & 0.435046 & -0.109570 \\
\hline 4 & 6 & 0 & -1.828331 & -0.698298 & 2.325920 \\
\hline 5 & 6 & 0 & -2.265969 & 0.254733 & 1.378651 \\
\hline 6 & 6 & 0 & -3.488034 & 0.919030 & 1.552367 \\
\hline 7 & 6 & 0 & -4.257375 & 0.705897 & 2.690594 \\
\hline 8 & 6 & 0 & -3.809556 & -0.207213 & 3.651645 \\
\hline 9 & 6 & 0 & -2.623817 & -0.906791 & 3.469692 \\
\hline 10 & 6 & 0 & -1.084106 & 2.190463 & -0.543333 \\
\hline 11 & 6 & 0 & -0.289351 & 2.473025 & -1.667204 \\
\hline 12 & 6 & 0 & 0.044376 & 3.781965 & -2.000748 \\
\hline 13 & 6 & 0 & -0.402056 & 4.837661 & -1.202090 \\
\hline 14 & 6 & 0 & -1.189884 & 4.570308 & -0.084948 \\
\hline 15 & 6 & 0 & -1.532791 & 3.256986 & 0.243161 \\
\hline 16 & 6 & 0 & -2.896424 & -1.528936 & -1.133053 \\
\hline 17 & 6 & 0 & -2.401208 & -0.236669 & -1.372827 \\
\hline 18 & 6 & 0 & -2.764847 & 0.421131 & -2.552629 \\
\hline 19 & 6 & 0 & -3.594021 & -0.207862 & -3.482949 \\
\hline 20 & 6 & 0 & -4.069893 & -1.495242 & -3.243936 \\
\hline 21 & 6 & 0 & -3.723730 & -2.153035 & -2.061276 \\
\hline 22 & 6 & 0 & 2.215889 & -2.556643 & -0.065895 \\
\hline 23 & 6 & 0 & 3.003530 & -1.471360 & -0.566631 \\
\hline 24 & 6 & 0 & 2.287525 & -0.855090 & -1.636242 \\
\hline 25 & 6 & 0 & 1.074328 & -1.600735 & -1.837055 \\
\hline 26 & 6 & 0 & 1.031042 & -2.657037 & -0.875394 \\
\hline 27 & 6 & 0 & 4.380223 & -1.115913 & -0.137748 \\
\hline 28 & 6 & 0 & 2.801355 & 0.218286 & -2.532938 \\
\hline 29 & 6 & 0 & 0.172762 & -1.431558 & -3.011039 \\
\hline 30 & 6 & 0 & 0.021286 & -3.748020 & -0.793800 \\
\hline 31 & 6 & 0 & 2.602553 & -3.497806 & 1.022474 \\
\hline 32 & 1 & 0 & -3.845380 & 1.601765 & 0.775136 \\
\hline 33 & 1 & 0 & -5.201983 & 1.236092 & 2.825181 \\
\hline 34 & 1 & 0 & -4.402639 & -0.384549 & 4.552320 \\
\hline 35 & 1 & 0 & -2.292853 & -1.633733 & 4.214787 \\
\hline 36 & 1 & 0 & 0.084850 & 1.646065 & -2.279956 \\
\hline 37 & 1 & 0 & 0.660972 & 3.978953 & -2.881333 \\
\hline 38 & 1 & 0 & -0.133777 & 5.866391 & -1.452122 \\
\hline 39 & 1 & 0 & -1.540666 & 5.390357 & 0.545595 \\
\hline 40 & 1 & 0 & -2.138322 & 3.068353 & 1.132057 \\
\hline & & & & & \\
\hline
\end{tabular}




\begin{tabular}{|c|c|c|c|c|c|}
\hline 41 & 1 & 0 & -2.626491 & -2.046190 & -0.205646 \\
\hline 42 & 1 & 0 & -2.407476 & 1.434558 & -2.751946 \\
\hline 43 & 1 & 0 & -3.872857 & 0.317753 & -4.399198 \\
\hline 44 & 1 & 0 & -4.718384 & -1.984667 & -3.973973 \\
\hline 45 & 1 & 0 & -4.103448 & -3.157637 & -1.860284 \\
\hline 46 & 1 & 0 & 4.637772 & -0.066296 & -0.338128 \\
\hline 47 & 1 & 0 & 4.552756 & -1.309759 & 0.927398 \\
\hline 48 & 1 & 0 & 5.109137 & -1.722548 & -0.698861 \\
\hline 49 & 1 & 0 & 1.999368 & 0.857220 & -2.929837 \\
\hline 50 & 1 & 0 & 3.544554 & 0.864204 & -2.042927 \\
\hline 51 & 1 & 0 & 3.309744 & -0.215638 & -3.410669 \\
\hline 52 & 1 & 0 & -0.799984 & -1.920809 & -2.877973 \\
\hline 53 & 1 & 0 & -0.020464 & -0.376260 & -3.252992 \\
\hline 54 & 1 & 0 & 0.638875 & -1.874353 & -3.906488 \\
\hline 55 & 1 & 0 & -0.258566 & -3.974927 & 0.244832 \\
\hline 56 & 1 & 0 & -0.900334 & -3.494474 & -1.334262 \\
\hline 57 & 1 & 0 & 0.412892 & -4.677385 & -1.238442 \\
\hline 58 & 1 & 0 & 3.247950 & -3.016481 & 1.768932 \\
\hline 59 & 1 & 0 & 1.724589 & -3.892082 & 1.552795 \\
\hline 60 & 1 & 0 & 3.154339 & -4.364231 & 0.622267 \\
\hline 61 & 5 & 0 & 3.060930 & 0.156710 & 2.673804 \\
\hline 62 & 1 & 0 & 1.217831 & 2.087293 & 0.803648 \\
\hline 63 & 1 & 0 & 3.249893 & 1.031789 & 3.492129 \\
\hline 64 & 6 & 0 & 1.080095 & 1.246261 & 1.512158 \\
\hline 65 & 7 & 0 & 2.164778 & 0.395699 & 1.634104 \\
\hline 66 & 6 & 0 & 0.352909 & 1.657768 & 2.760602 \\
\hline 67 & 1 & 0 & -0.609132 & 2.149483 & 2.559373 \\
\hline 68 & 1 & 0 & 0.169070 & 0.797358 & 3.418598 \\
\hline 69 & 1 & 0 & 0.966971 & 2.374314 & 3.333086 \\
\hline 70 & 1 & 0 & 3.671839 & -0.880994 & 2.753660 \\
\hline 71 & 7 & 0 & 3.728686 & 2.660656 & 0.168638 \\
\hline 72 & 1 & 0 & 3.032610 & 3.007021 & -0.492346 \\
\hline 73 & 1 & 0 & 3.416223 & 1.729705 & 0.475996 \\
\hline 74 & 1 & 0 & 3.659485 & 3.252577 & 0.996682 \\
\hline 75 & 5 & 0 & 5.237580 & 2.656879 & -0.447217 \\
\hline 76 & 1 & 0 & 5.185801 & 1.964258 & -1.454397 \\
\hline 77 & 1 & 0 & 5.506323 & 3.819980 & -0.695954 \\
\hline 78 & 1 & 0 & 5.940779 & 2.166395 & 0.421151 \\
\hline
\end{tabular}

\section{E-TS3(triplet)opt $=-\mathbf{2 1 3 3 . 9 3 7 5 3 6 9 h a r t r e e}$}

$\begin{array}{lccccc}\text { Center } & \text { Atomic } & \text { Atomic } & \text { Coordinates (Angstroms) } & \\ \text { Number } & \text { Number } & \text { Type } & X & Y & \text { Z }\end{array}$




\begin{tabular}{|c|c|c|c|c|c|}
\hline 1 & 42 & 0 & 1.062539 & -0.359064 & 0.128941 \\
\hline 2 & 16 & 0 & 0.342386 & -0.612637 & 2.470541 \\
\hline 3 & 15 & 0 & -1.322065 & 0.316365 & -0.076587 \\
\hline 4 & 6 & 0 & -1.203360 & 0.177933 & 2.666989 \\
\hline 5 & 6 & 0 & -1.981837 & 0.623182 & 1.573989 \\
\hline 6 & 6 & 0 & -3.268055 & 1.141157 & 1.786991 \\
\hline 7 & 6 & 0 & -3.769945 & 1.297791 & 3.073530 \\
\hline 8 & 6 & 0 & -2.983529 & 0.902643 & 4.161944 \\
\hline 9 & 6 & 0 & -1.729318 & 0.342099 & 3.964478 \\
\hline 10 & 6 & 0 & -1.559327 & 1.778246 & -1.136153 \\
\hline 11 & 6 & 0 & -1.011794 & 1.709008 & -2.427777 \\
\hline 12 & 6 & 0 & -1.075714 & 2.795318 & -3.294495 \\
\hline 13 & 6 & 0 & -1.673993 & 3.985129 & -2.875470 \\
\hline 14 & 6 & 0 & -2.205417 & 4.072496 & -1.591179 \\
\hline 15 & 6 & 0 & -2.153309 & 2.977068 & -0.727195 \\
\hline 16 & 6 & 0 & -2.809250 & -1.999425 & 0.075842 \\
\hline 17 & 6 & 0 & -2.560903 & -0.868696 & -0.718553 \\
\hline 18 & 6 & 0 & -3.226345 & -0.744146 & -1.943327 \\
\hline 19 & 6 & 0 & -4.108576 & -1.737316 & -2.371903 \\
\hline 20 & 6 & 0 & -4.338087 & -2.862647 & -1.583121 \\
\hline 21 & 6 & 0 & -3.689137 & -2.988373 & -0.352763 \\
\hline 22 & 6 & 0 & 2.404903 & -2.298439 & 0.274153 \\
\hline 23 & 6 & 0 & 3.023898 & -1.446403 & -0.685953 \\
\hline 24 & 6 & 0 & 2.108504 & -1.244885 & -1.765923 \\
\hline 25 & 6 & 0 & 0.936163 & -2.035753 & -1.495261 \\
\hline 26 & 6 & 0 & 1.120653 & -2.688822 & -0.235401 \\
\hline 27 & 6 & 0 & 4.422433 & -0.947446 & -0.614725 \\
\hline 28 & 6 & 0 & 2.385871 & -0.487202 & -3.020642 \\
\hline 29 & 6 & 0 & -0.158991 & -2.300475 & -2.471809 \\
\hline 30 & 6 & 0 & 0.236866 & -3.704191 & 0.401269 \\
\hline 31 & 6 & 0 & 3.022007 & -2.802725 & 1.532039 \\
\hline 32 & 1 & 0 & -3.890854 & 1.406081 & 0.927036 \\
\hline 33 & 1 & 0 & -4.767704 & 1.712336 & 3.229440 \\
\hline 34 & 1 & 0 & -3.362888 & 1.021740 & 5.180005 \\
\hline 35 & 1 & 0 & -1.131310 & 0.013760 & 4.817518 \\
\hline 36 & 1 & 0 & -0.511690 & 0.788609 & -2.748409 \\
\hline 37 & 1 & 0 & -0.646821 & 2.717563 & -4.296373 \\
\hline 38 & 1 & 0 & -1.717470 & 4.844651 & -3.547995 \\
\hline 39 & 1 & 0 & -2.664419 & 5.003467 & -1.250868 \\
\hline 40 & 1 & 0 & -2.562914 & 3.071891 & 0.280535 \\
\hline 41 & 1 & 0 & -2.303191 & -2.100263 & 1.042110 \\
\hline 42 & 1 & 0 & -3.063214 & 0.134950 & -2.571820 \\
\hline 43 & 1 & 0 & -4.623411 & -1.625146 & -3.329062 \\
\hline 44 & 1 & 0 & -5.029267 & -3.638196 & -1.920750 \\
\hline
\end{tabular}




\begin{tabular}{|c|c|c|c|c|c|}
\hline 45 & 1 & 0 & -3.872664 & -3.861462 & 0.278185 \\
\hline 46 & 1 & 0 & 4.668860 & -0.300226 & -1.465864 \\
\hline 47 & 1 & 0 & 4.633994 & -0.394181 & 0.311684 \\
\hline 48 & 1 & 0 & 5.128309 & -1.791806 & -0.646080 \\
\hline 49 & 1 & 0 & 1.467226 & -0.291897 & -3.590635 \\
\hline 50 & 1 & 0 & 2.857624 & 0.488185 & -2.827180 \\
\hline 51 & 1 & 0 & 3.063564 & -1.039846 & -3.693285 \\
\hline 52 & 1 & 0 & -1.029789 & -2.778779 & -2.006409 \\
\hline 53 & 1 & 0 & -0.521836 & -1.391227 & -2.973318 \\
\hline 54 & 1 & 0 & 0.195757 & -2.977023 & -3.266329 \\
\hline 55 & 1 & 0 & 0.058103 & -3.481945 & 1.464245 \\
\hline 56 & 1 & 0 & -0.739951 & -3.772897 & -0.093830 \\
\hline 57 & 1 & 0 & 0.695814 & -4.705158 & 0.353303 \\
\hline 58 & 1 & 0 & 3.822392 & -2.140759 & 1.886607 \\
\hline 59 & 1 & 0 & 2.284803 & -2.889806 & 2.343141 \\
\hline 60 & 1 & 0 & 3.457967 & -3.804594 & 1.383642 \\
\hline 61 & 5 & 0 & 3.220392 & 1.214205 & 1.816930 \\
\hline 62 & 1 & 0 & 1.042759 & 2.328429 & -0.580895 \\
\hline 63 & 1 & 0 & 2.860087 & 1.913065 & 2.735479 \\
\hline 64 & 6 & 0 & 1.163994 & 1.853849 & 0.414085 \\
\hline 65 & 7 & 0 & 2.389129 & 1.211248 & 0.563129 \\
\hline 66 & 6 & 0 & 0.583872 & 2.688795 & 1.519526 \\
\hline 67 & 1 & 0 & -0.442582 & 3.010158 & 1.294238 \\
\hline 68 & 1 & 0 & 0.578411 & 2.167912 & 2.484409 \\
\hline 69 & 1 & 0 & 1.182804 & 3.605424 & 1.657034 \\
\hline 70 & 1 & 0 & 3.736134 & 0.155570 & 2.113946 \\
\hline 71 & 7 & 0 & 4.214105 & 2.383980 & -0.742583 \\
\hline 72 & 1 & 0 & 4.676162 & 1.977843 & -1.551044 \\
\hline 73 & 1 & 0 & 3.287307 & 1.704519 & -0.348228 \\
\hline 74 & 1 & 0 & 3.848776 & 3.290551 & -1.024703 \\
\hline 75 & 5 & 0 & 5.108228 & 2.437144 & 0.534373 \\
\hline 76 & 1 & 0 & 6.054972 & 1.683739 & 0.448620 \\
\hline 77 & 1 & 0 & 5.342546 & 3.566562 & 0.905625 \\
\hline 78 & 1 & 0 & 4.384337 & 1.965615 & 1.501485 \\
\hline
\end{tabular}

\section{E-Int6(singlet)opt $=\mathbf{- 2 0 5 1 . 9 9 9 2 7 4 5 h a r t r e e}$}

\begin{tabular}{|c|c|c|c|c|c|}
\hline \multirow{2}{*}{$\begin{array}{l}\text { Center } \\
\text { Number }\end{array}$} & \multirow{2}{*}{$\begin{array}{l}\text { Atomic } \\
\text { Number }\end{array}$} & \multirow{2}{*}{$\begin{array}{l}\text { Atomic } \\
\text { Type }\end{array}$} & \multicolumn{3}{|c|}{ Coordinates (Angstroms) } \\
\hline & & & $\mathrm{X}$ & $\mathrm{Y}$ & $z$ \\
\hline 1 & 42 & 0 & -1.447146 & -0.155572 & -0.318943 \\
\hline 2 & 16 & 0 & -0.758201 & -2.499499 & -0.474800 \\
\hline 3 & 15 & 0 & 1.035584 & 0.105169 & -0.067650 \\
\hline 4 & 6 & 0 & 0.936853 & -2.532677 & -0.867276 \\
\hline & & & & & \\
\hline
\end{tabular}




\begin{tabular}{|c|c|c|c|c|c|}
\hline 5 & 6 & 0 & 1.779811 & -1.402690 & -0.744843 \\
\hline 6 & 6 & 0 & 3.165965 & -1.549287 & -0.932117 \\
\hline 7 & 6 & 0 & 3.716563 & -2.756500 & -1.340383 \\
\hline 8 & 6 & 0 & 2.873282 & -3.859797 & -1.527469 \\
\hline 9 & 6 & 0 & 1.514365 & -3.754395 & -1.280155 \\
\hline 10 & 6 & 0 & 1.676501 & 1.588501 & -0.960204 \\
\hline 11 & 6 & 0 & 0.954654 & 2.778153 & -0.780206 \\
\hline 12 & 6 & 0 & 1.342053 & 3.961123 & -1.406625 \\
\hline 13 & 6 & 0 & 2.454116 & 3.972385 & -2.247843 \\
\hline 14 & 6 & 0 & 3.164819 & 2.792002 & -2.456668 \\
\hline 15 & 6 & 0 & 2.783569 & 1.611778 & -1.817912 \\
\hline 16 & 6 & 0 & 2.373005 & -0.940807 & 2.165064 \\
\hline 17 & 6 & 0 & 2.043792 & 0.237185 & 1.474404 \\
\hline 18 & 6 & 0 & 2.508324 & 1.456999 & 1.986454 \\
\hline 19 & 6 & 0 & 3.253273 & 1.501900 & 3.165082 \\
\hline 20 & 6 & 0 & 3.556434 & 0.326089 & 3.848492 \\
\hline 21 & 6 & 0 & 3.118088 & -0.896959 & 3.339586 \\
\hline 22 & 6 & 0 & -2.765974 & -0.875760 & 1.422337 \\
\hline 23 & 6 & 0 & -3.578129 & 0.108769 & 0.779978 \\
\hline 24 & 6 & 0 & -2.897410 & 1.358075 & 0.821221 \\
\hline 25 & 6 & 0 & -1.681294 & 1.187531 & 1.550091 \\
\hline 26 & 6 & 0 & -1.600253 & -0.210253 & 1.937861 \\
\hline 27 & 6 & 0 & -4.958460 & -0.146099 & 0.289230 \\
\hline 28 & 6 & 0 & -3.396368 & 2.653372 & 0.282659 \\
\hline 29 & 6 & 0 & -0.906954 & 2.298467 & 2.170906 \\
\hline 30 & 6 & 0 & -0.719022 & -0.790861 & 2.989818 \\
\hline 31 & 6 & 0 & -3.182946 & -2.270421 & 1.730671 \\
\hline 32 & 1 & 0 & 3.832083 & -0.711206 & -0.711904 \\
\hline 33 & 1 & 0 & 4.793663 & -2.846288 & -1.494613 \\
\hline 34 & 1 & 0 & 3.290021 & -4.817988 & -1.848653 \\
\hline 35 & 1 & 0 & 0.863473 & -4.625116 & -1.390885 \\
\hline 36 & 1 & 0 & 0.059495 & 2.757397 & -0.152107 \\
\hline 37 & 1 & 0 & 0.765580 & 4.875535 & -1.244858 \\
\hline 38 & 1 & 0 & 2.757263 & 4.894189 & -2.749243 \\
\hline 39 & 1 & 0 & 4.024264 & 2.783242 & -3.131166 \\
\hline 40 & 1 & 0 & 3.340721 & 0.698102 & -2.026403 \\
\hline 41 & 1 & 0 & 2.040420 & -1.906918 & 1.776085 \\
\hline 42 & 1 & 0 & 2.299851 & 2.389312 & 1.455501 \\
\hline 43 & 1 & 0 & 3.608201 & 2.463894 & 3.542390 \\
\hline 44 & 1 & 0 & 4.143709 & 0.360553 & 4.768989 \\
\hline 45 & 1 & 0 & 3.361476 & -1.826597 & 3.859312 \\
\hline 46 & 1 & 0 & -5.365012 & 0.693998 & -0.283865 \\
\hline 47 & 1 & 0 & -5.022396 & -1.041750 & -0.342406 \\
\hline 48 & 1 & 0 & -5.620297 & -0.314932 & 1.154308 \\
\hline
\end{tabular}




\begin{tabular}{llllll}
\hline 49 & 1 & 0 & -2.570114 & 3.312652 & -0.020895 \\
50 & 1 & 0 & -4.042993 & 2.511727 & -0.593018 \\
51 & 1 & 0 & -3.979372 & 3.202176 & 1.039737 \\
52 & 1 & 0 & 0.163364 & 2.081548 & 2.269245 \\
53 & 1 & 0 & -1.012479 & 3.243311 & 1.621380 \\
54 & 1 & 0 & -1.280848 & 2.488788 & 3.191613 \\
55 & 1 & 0 & -0.256659 & -1.738436 & 2.678065 \\
56 & 1 & 0 & 0.082902 & -0.103986 & 3.285889 \\
57 & 1 & 0 & -1.307092 & -1.005299 & 3.897714 \\
58 & 1 & 0 & -3.752442 & -2.721423 & 0.906286 \\
59 & 1 & 0 & -2.316728 & -2.918098 & 1.916293 \\
60 & 1 & 0 & -3.823157 & -2.301988 & 2.628385 \\
61 & 5 & 0 & -3.026937 & 0.209799 & -2.282996 \\
62 & 1 & 0 & 0.360476 & 0.637914 & -2.463311 \\
63 & 1 & 0 & -3.207129 & -0.385168 & -3.327670 \\
64 & 6 & 0 & -0.561771 & 0.061092 & -2.364617 \\
65 & 7 & -1.672977 & 0.920505 & -2.152099 \\
66 & 6 & 0 & -0.610953 & -1.030903 & -3.395899 \\
67 & 1 & 0 & -3.334854 & -1.589916 & -3.366888 \\
68 & 1 & 0 & -1.420141 & -1.753225 & -3.244266 \\
69 & 1 & 0 & -0.711307 & -0.612788 & -4.411705 \\
70 & 1 & 0 & -2.858454 & -0.810811 & -1.477316 \\
71 & 1 & 0 & -1.540427 & 1.921783 & -2.270451 \\
72 & 1 & 0 & -3.947235 & 0.915698 & -1.942560 \\
\hline & & 0 & &
\end{tabular}

E-TS1'(singlet)opt $=\mathbf{- 1 9 7 4 . 6 0 5 4 9 1 1}$ hartree

\begin{tabular}{rrrrrr}
$\begin{array}{c}\text { Center } \\
\text { Number }\end{array}$ & $\begin{array}{c}\text { Atomic } \\
\text { Number }\end{array}$ & $\begin{array}{r}\text { Atomic } \\
\text { Type }\end{array}$ & \multicolumn{3}{c}{ Coordinates (Angstroms) } \\
\hline 1 & 42 & & X & Z \\
2 & 16 & 0 & -0.559841 & 2.090208 & -1.193630 \\
3 & 15 & 0 & 1.319928 & 0.101154 & 0.206677 \\
4 & 6 & 0 & 1.078297 & 2.592179 & -0.808142 \\
5 & 6 & 0 & 2.008479 & 1.693872 & -0.240543 \\
6 & 6 & 0 & 3.333451 & 2.085975 & -0.014405 \\
7 & 6 & 0 & 3.747986 & 3.375369 & -0.334186 \\
8 & 6 & 0 & 2.826366 & 4.276464 & -0.879881 \\
9 & 6 & 0 & 1.510064 & 3.895647 & -1.112674 \\
10 & 6 & 0 & 0.336174 & 0.311473 & 1.645619 \\
11 & 6 & 0 & -0.558387 & -0.815518 & 1.929377 \\
12 & 6 & 0 & -1.499711 & -0.632578 & 3.007249 \\
13 & 6 & 0 & -1.564543 & 0.529593 & 3.724924 \\
14 & 6 & 0 & -0.646693 & 1.600832 & 3.464107
\end{tabular}


15

16

17

18

19

20

21

22

23

24

25

26

27

28

29

30

31

32

33

34

35

36

37

38

39

40

41

42

43

44

45

46

47

48

49

50

51

52

53

54

55

56

57

58
0.273513

3.536632

2.678952

2.823280

3.797758

4.642354

4.515924

$-0.840706$

$-2.150861$

$-2.897529$

$-2.083611$

$-0.802445$

$-2.682093$

$-4.300349$

$-2.511848$

0.262255

0.203925

4.039669

4.779913

3.143346

0.797767

$-0.187500$

$-2.146888$

$-2.281352$

$-0.675597$

0.981010

3.436704

2.170850

3.903403

5.406737

5.183509

$-3.318009$

$-1.872804$

$-3.289156$

$-4.522310$

$-4.506082$

$-5.018386$

$-1.683096$

$-3.314170$

$-2.894278$

1.227848

0.432871

0.001517

0.319913
1.496480

$-1.306619$

$-1.076403$

$-1.849169$

$-2.844206$

$-3.070771$

$-2.293662$

$-1.211767$

$-0.607323$

$-1.218383$

$-2.197691$

$-2.205367$

0.379367

$-0.876928$

$-3.133742$

$-3.222531$

$-0.977446$

1.374104

3.682445

5.292050

4.600939

$-1.840083$

$-1.474547$

0.625167

2.499196

2.308837

$-0.701801$

$-1.660565$

$-3.441637$

$-3.849356$

$-2.460010$

1.137043

0.919184

$-0.115586$

$-1.124619$

0.193006

$-1.433812$

$-3.406550$

$-2.713974$

$-4.076186$

$-2.916202$

$-3.413058$

$-4.192189$

0.093546
2.462674

$-0.712116$

0.375103

1.535264

1.599082

0.512667

$-0.640386$

$-2.138041$

$-2.117237$

$-1.062929$

$-0.429693$

$-1.077028$

$-3.098339$

$-0.699929$

0.644772

$-0.842981$

$-3.176468$

0.424024

$-0.152211$

$-1.130967$

$-1.547482$

1.791439

3.276113

4.546412

4.085648

2.271213

$-1.619437$

2.392089

2.507729

0.565997

$-1.489136$

$-2.617502$

$-3.606225$

$-3.874521$

0.347925

$-0.843715$

$-1.323647$

1.313226

1.266933

0.218580

$-1.266968$

0.227897

$-1.300237$

$-3.398647$ 


\begin{tabular}{rrrrrr}
\hline 59 & 1 & 0 & 1.184870 & -1.347610 & -2.846727 \\
60 & 1 & 0 & -0.033901 & -1.489311 & -4.124265 \\
61 & 5 & 0 & -2.398922 & 1.996665 & 0.350245 \\
62 & 1 & 0 & -2.223465 & 0.832078 & 0.800116 \\
63 & 1 & 0 & -3.139139 & 2.176181 & -0.580345 \\
64 & 1 & 0 & -2.003752 & 2.868592 & 1.071555 \\
65 & 7 & 0 & -4.161550 & 1.760822 & 1.791929 \\
66 & 1 & 0 & -4.975528 & 1.176302 & 1.611457 \\
67 & 1 & 0 & -4.504791 & 2.697777 & 1.991346 \\
68 & 1 & 0 & -3.724171 & 1.413944 & 2.645881
\end{tabular}

\section{E-Int0 $($ triplet)opt $=\mathbf{- 1 9 1 8 . 0 9 5 3 3 4 6}$ hartree}

\begin{tabular}{|c|c|c|c|c|c|}
\hline \multirow{2}{*}{$\begin{array}{l}\text { Center } \\
\text { Number }\end{array}$} & \multirow{2}{*}{$\begin{array}{l}\text { Atomic } \\
\text { Number }\end{array}$} & \multirow{2}{*}{$\begin{array}{c}\text { Atomic } \\
\text { Type }\end{array}$} & \multicolumn{3}{|c|}{ Coordinates (Angstroms) } \\
\hline & & & $\mathrm{X}$ & $\mathrm{Y}$ & Z \\
\hline 1 & 5 & 0 & 0.836907 & 2.033233 & -2.059606 \\
\hline 2 & 1 & 0 & 1.850003 & 2.557175 & -2.469922 \\
\hline 3 & 1 & 0 & -0.173212 & 2.236184 & -2.700137 \\
\hline 4 & 6 & 0 & 2.320204 & -1.425314 & 0.823865 \\
\hline 5 & 6 & 0 & 2.817416 & -1.435048 & -0.520575 \\
\hline 6 & 6 & 0 & 3.529770 & -0.207501 & -0.752137 \\
\hline 7 & 6 & 0 & 3.480355 & 0.577203 & 0.433348 \\
\hline 8 & 6 & 0 & 2.718413 & -0.166999 & 1.409776 \\
\hline 9 & 6 & 0 & 1.666883 & -2.557815 & 1.540103 \\
\hline 10 & 1 & 0 & 0.950212 & -2.210642 & 2.298524 \\
\hline 11 & 1 & 0 & 2.405415 & -3.191093 & 2.060589 \\
\hline 12 & 1 & 0 & 1.110506 & -3.212175 & 0.854159 \\
\hline 13 & 6 & 0 & 2.688021 & -2.552313 & -1.499276 \\
\hline 14 & 1 & 0 & 1.864152 & -3.230662 & -1.240280 \\
\hline 15 & 1 & 0 & 3.605663 & -3.163281 & -1.536581 \\
\hline 16 & 1 & 0 & 2.504344 & -2.193806 & -2.522568 \\
\hline 17 & 6 & 0 & 4.214296 & 0.165446 & -2.021588 \\
\hline 18 & 1 & 0 & 3.692209 & -0.230126 & -2.903672 \\
\hline 19 & 1 & 0 & 5.242119 & -0.231706 & -2.051534 \\
\hline 20 & 1 & 0 & 4.280454 & 1.253839 & -2.147382 \\
\hline 21 & 6 & 0 & 4.159840 & 1.882498 & 0.663329 \\
\hline 22 & 1 & 0 & 4.233028 & 2.475041 & -0.258885 \\
\hline 23 & 1 & 0 & 5.185627 & 1.745114 & 1.044744 \\
\hline 24 & 1 & 0 & 3.624583 & 2.498049 & 1.398976 \\
\hline 25 & 6 & 0 & 2.497421 & 0.245361 & 2.827104 \\
\hline 26 & 1 & 0 & 2.282157 & 1.320074 & 2.920195 \\
\hline 27 & 1 & 0 & 3.385894 & 0.043707 & 3.449417 \\
\hline 28 & 1 & 0 & 1.658542 & -0.297945 & 3.283690 \\
\hline
\end{tabular}




\begin{tabular}{|c|c|c|c|c|c|}
\hline 29 & 6 & 0 & -1.230681 & 2.659567 & -0.036099 \\
\hline 30 & 6 & 0 & -1.985118 & 1.483253 & 0.063544 \\
\hline 31 & 6 & 0 & -3.367829 & 1.566852 & 0.271048 \\
\hline 32 & 1 & 0 & -3.954786 & 0.647128 & 0.365888 \\
\hline 33 & 6 & 0 & -3.996230 & 2.805830 & 0.363606 \\
\hline 34 & 1 & 0 & -5.074615 & 2.862661 & 0.52770 \\
\hline 35 & 6 & 0 & -3.238976 & 3.974550 & 0.253075 \\
\hline 36 & 1 & 0 & -3.725708 & 4.950136 & 0.326550 \\
\hline 37 & 6 & 0 & -1.861746 & 3.904023 & 0.057150 \\
\hline 38 & 1 & 0 & -1.264822 & 4.815984 & -0.022489 \\
\hline 39 & 6 & 0 & -1.645660 & -0.962418 & 1.463419 \\
\hline 40 & 6 & 0 & -2.346736 & -2.173465 & 1.467746 \\
\hline 41 & 1 & 0 & -2.661625 & -2.628884 & 0.525343 \\
\hline 42 & 6 & 0 & -2.652235 & -2.807855 & 2.673435 \\
\hline 43 & 1 & 0 & -3.197670 & -3.754619 & 2.661336 \\
\hline 44 & 6 & 0 & -2.271953 & -2.237606 & 3.8863 \\
\hline 45 & 1 & 0 & -2.512353 & -2.737244 & 4.827535 \\
\hline 46 & 6 & 0 & -1.587649 & -1.019912 & 3.892961 \\
\hline 47 & 1 & 0 & -1.294310 & -0.559803 & 4.839824 \\
\hline 48 & 6 & 0 & -1.274306 & -0.391384 & 2.692066 \\
\hline 49 & 1 & 0 & -0.728498 & 0.559102 & 2.699936 \\
\hline 50 & 6 & 0 & -1.795658 & -0.995704 & -1.440857 \\
\hline 51 & 6 & 0 & -2.687734 & -0.416370 & -2.351650 \\
\hline 52 & 1 & 0 & -3.047285 & 0.602938 & -2.192648 \\
\hline 53 & 6 & 0 & -3.106579 & -1.126586 & -3.477372 \\
\hline 54 & 1 & 0 & -3.796673 & -0.658242 & -4.182947 \\
\hline 55 & 6 & 0 & -2.646310 & -2.421282 & -3.706297 \\
\hline 56 & 1 & 0 & -2.976751 & -2.973522 & -4.588951 \\
\hline 57 & 6 & 0 & -1.751239 & -3.006250 & -2.807783 \\
\hline 58 & 1 & 0 & -1.377952 & -4.017924 & -2.984021 \\
\hline 59 & 6 & 0 & -1.322074 & -2.294881 & -1.692336 \\
\hline 60 & 1 & 0 & -0.599675 & -2.743133 & -1.002177 \\
\hline 61 & 42 & 0 & 1.299035 & 0.286416 & -0.27317 \\
\hline 62 & 1 & 0 & 0.946700 & 0.748751 & -2.099622 \\
\hline 63 & 15 & 0 & -1.070426 & -0.095572 & -0.037047 \\
\hline 64 & 16 & 0 & 0.558328 & 2.597601 & -0.2318 \\
\hline
\end{tabular}

\section{E-TS2"(singlet)opt $=\mathbf{- 1 9 1 8 . 0 8 8 9 0 2 7 h a r t r e e}$}

\begin{tabular}{cccccc}
$\begin{array}{c}\text { Center } \\
\text { Number }\end{array}$ & $\begin{array}{c}\text { Atomic } \\
\text { Number }\end{array}$ & $\begin{array}{c}\text { Atomic } \\
\text { Type }\end{array}$ & \multicolumn{3}{c}{ Coordinates (Angstroms) } \\
-1 & 5 & 0 & -0.562178 & -0.648207 & -2.482555 \\
2 & 1 & 0 & -1.841414 & -0.786462 & -2.443074
\end{tabular}


3

4

5

6

7

8

9

10

11

12

13

14

15

16

17

18

19

20

21

22

23

24

25

26

27

28

29

30

31

32

33

34

35

36

37

38

39

40

41

42

43

44

45

46
0.040870

$-2.131956$

$-2.707519$

$-3.482287$

$-3.353596$

$-2.502701$

$-1.409795$

$-0.842807$

$-2.133855$

$-0.706683$

$-2.648279$

$-1.797928$

$-3.557542$

$-2.558523$

$-4.383774$

$-3.988328$

$-5.382652$

$-4.533152$

$-4.130573$

$-4.392783$

$-5.075952$

$-3.565129$

$-2.224411$

$-1.903252$

$-3.122286$

$-1.437868$

1.258267

1.942172

3.332361

3.850739

4.048884

5.129241

3.368184

3.921566

1.993188

1.463142

1.460157

2.030052

2.234310

2.351082

2.795029

2.115290

2.368690

1.565862
$-0.818813$

0.804396

1.499459

0.563107

$-0.739231$

$-0.574148$

1.468779

0.766687

1.975265

2.240358

2.968692

3.436762

3.472100

3.211192

0.905690

1.737039

1.208695

0.056765

$-1.970348$

$-2.021838$

$-2.018035$

$-2.882317$

$-1.636696$

$-2.577767$

$-1.867787$

$-1.337430$

$-2.477068$

$-1.328504$

$-1.373727$

$-0.485184$

$-2.534141$

$-2.560614$

$-3.673677$

$-4.593752$

$-3.648860$

$-4.539989$

0.324282

1.470382

2.346157

1.505195

2.408198

0.395472

0.425664

$-0.761141$
$-3.496952$

1.389646

0.286088

$-0.472478$

0.159405

1.313822

2.508751

3.131642

3.167863

2.163051

0.045034

0.558836

0.414909

$-1.022936$

$-1.610790$

$-2.210093$

$-1.253914$

$-2.291677$

$-0.168567$

$-1.234241$

0.398524

0.067036

2.321630

1.851120

2.918892

3.027019

$-0.965025$

$-0.529427$

$-0.333511$

0.041820

$-0.593609$

$-0.437484$

$-1.043841$

$-1.248239$

$-1.226680$

$-1.571547$

1.646649

2.209521

1.588866

3.568252

3.994187

4.376464

5.438647

3.818290 


$\begin{array}{rrrrrr}47 & 1 & 0 & 1.391641 & -1.642401 & 4.440639 \\ 48 & 6 & 0 & 1.242241 & -0.794984 & 2.465784 \\ 49 & 1 & 0 & 0.815266 & -1.703746 & 2.028500 \\ 50 & 6 & 0 & 1.741786 & 1.550013 & -0.945240 \\ 51 & 6 & 0 & 2.729347 & 1.417730 & -1.927657 \\ 52 & 1 & 0 & 3.122856 & 0.429868 & -2.178735 \\ 53 & 6 & 0 & 3.202308 & 2.539792 & -2.610202 \\ 54 & 1 & 0 & 3.969336 & 2.418582 & -3.378709 \\ 55 & 6 & 0 & 2.699295 & 3.805792 & -2.319686 \\ 56 & 1 & 0 & 3.072128 & 4.681665 & -2.855562 \\ 57 & 6 & 0 & 1.704478 & 3.947802 & -1.350211 \\ 58 & 1 & 0 & 1.291825 & 4.934421 & -1.126221 \\ 59 & 6 & 0 & 1.222677 & 2.827194 & -0.681336 \\ 60 & 1 & 0 & 0.415080 & 2.934824 & 0.050459 \\ 61 & 42 & 0 & -1.389945 & -0.179985 & -0.705034 \\ 62 & 1 & 0 & -0.759140 & 0.786759 & -2.086975 \\ 63 & 15 & 0 & 0.959633 & 0.131855 & -0.104886 \\ 64 & 16 & 0 & -0.480299 & -2.481237 & -1.177164\end{array}$

\section{E-Int0 $($ singlet $) o p t=-1918.1077527$ hartree}

\begin{tabular}{|c|c|c|c|c|c|}
\hline \multirow{2}{*}{$\begin{array}{l}\text { Center } \\
\text { Number }\end{array}$} & \multirow{2}{*}{$\begin{array}{l}\text { Atomic } \\
\text { Number }\end{array}$} & \multirow{2}{*}{$\begin{array}{l}\text { Atomic } \\
\text { Type }\end{array}$} & \multicolumn{3}{|c|}{ Coordinates (Angstroms) } \\
\hline & & & $\mathrm{X}$ & Y & $z$ \\
\hline 1 & 42 & 0 & 1.106289 & -0.140223 & -0.064268 \\
\hline 2 & 16 & 0 & 0.386379 & -2.227144 & -1.084054 \\
\hline 3 & 15 & 0 & -1.202727 & 0.118986 & 0.104783 \\
\hline 4 & 6 & 0 & -1.348049 & -2.461726 & -0.722422 \\
\hline 5 & 6 & 0 & -2.135384 & -1.364523 & -0.333162 \\
\hline 6 & 6 & 0 & -3.517925 & -1.517792 & -0.183404 \\
\hline 7 & 6 & 0 & -4.111564 & -2.759950 & -0.398006 \\
\hline 8 & 6 & 0 & -3.322243 & -3.854356 & -0.758582 \\
\hline 9 & 6 & 0 & -1.946816 & -3.707898 & -0.927793 \\
\hline 10 & 6 & 0 & -0.500120 & -0.229435 & 1.680029 \\
\hline 11 & 6 & 0 & 0.605455 & 0.636765 & 2.066521 \\
\hline 12 & 6 & 0 & 1.365675 & 0.254739 & 3.225611 \\
\hline 13 & 6 & 0 & 1.073594 & -0.874521 & 3.939663 \\
\hline 14 & 6 & 0 & -0.052442 & -1.678792 & 3.585881 \\
\hline 15 & 6 & 0 & -0.814390 & -1.372521 & 2.494651 \\
\hline 16 & 6 & 0 & -2.982546 & 1.844840 & -1.162109 \\
\hline 17 & 6 & 0 & -2.330044 & 1.527925 & 0.039491 \\
\hline 18 & 6 & 0 & -2.459507 & 2.389264 & 1.137375 \\
\hline 19 & 6 & 0 & -3.220891 & 3.551985 & 1.030454 \\
\hline 20 & 6 & 0 & -3.865456 & 3.861272 & -0.167160 \\
\hline
\end{tabular}




21
22
23
24
25
26
27
28
29
30
31
32
33
34
35
36
37
38
39
40
41
42
43
44
45
46
47
48
49
50
51
52
53
54
55
56
57
58
59
60
61
63

$-3.751232$

$3.001738-1.261326$

$\begin{array}{lll}1.549271 & 0.922293 & -1.941063\end{array}$

$\begin{array}{lll}2.655955 & 0.021447 & -1.707758\end{array}$

$\begin{array}{lll}3.342082 & 0.461567 & -0.528813\end{array}$

$\begin{array}{lll}2.691735 & 1.623587 & -0.033495\end{array}$

$\begin{array}{lll}1.571630 & 1.914804 & -0.881084\end{array}$

$3.108184 \quad-1.075200 \quad-2.610925$

$\begin{array}{lll}4.539401 & -0.197583 & 0.062051\end{array}$

$\begin{array}{lll}3.148862 & 2.457550 & 1.110286\end{array}$

$\begin{array}{lll}0.730531 & 3.143609 & -0.796517\end{array}$

$\begin{array}{lll}0.682654 & 0.944959 & -3.155116\end{array}$

$\begin{array}{lll}-4.127315 & -0.660985 & 0.119794\end{array}$

$\begin{array}{lll}-5.190005 & -2.878826 & -0.273256\end{array}$

$\begin{array}{lll}-3.784647 & -4.831099 & -0.919429\end{array}$

$\begin{array}{lll}-1.330342 & -4.557672 & -1.230273\end{array}$

$\begin{array}{lll}0.543526 & 1.710424 & 1.852976\end{array}$

$\begin{array}{lll}2.179250 & 0.910268 & 3.551282\end{array}$

$\begin{array}{lll}1.676009 & -1.144253 & 4.810093\end{array}$

$\begin{array}{lll}-0.309414 & -2.545169 & 4.199934\end{array}$

$\begin{array}{lll}-1.674372 & -1.995491 & 2.233066\end{array}$

$\begin{array}{lll}-2.887827 & 1.177368 & -2.025007\end{array}$

$\begin{array}{lll}-1.961730 & 2.139711 & 2.078642\end{array}$

$\begin{array}{lll}-3.317145 & 4.217831 & 1.891237\end{array}$

$-4.463256$

4.771934

$-0.247781$

$-4.261730$

3.236819

$-2.198200$

3.447454

$-1.958474$

$-2.050761$

2.304718

$-1.408034$

$-3.280598$

3.947914

$-0.748960$

$-3.246440$

4.605502

$-0.035386$

1.146570

4.527532

$-1.282966$

$-0.105865$

5.469719

0.189829

$-0.384334$

2.314192

2.925729

1.651129

3.736161

1.880298

1.836680

3.792310

3.280654

0.758384

$-0.223044$

3.030961

$-1.330724$

0.482500

3.409185

0.242582

1.242929

4.018304

$-1.232181$

0.416650

$-0.068540$

$-3.487694$

$-0.258863$

1.479711

$-2.966379$

1.175758

1.448050

$-4.004297$

1.534836

$-2.592808$

0.411813

1.855418

$-1.487343$

1.012545

0.998657

$-3.264750$

1.262313

2.555501

$-3.040480$

$-0.070376$ 
E-Int0c(singlet)opt $=\mathbf{- 1 9 7 4 . 6 2 6 1 7 2 7}$ hartree

\begin{tabular}{|c|c|c|c|c|c|}
\hline \multirow{2}{*}{$\begin{array}{l}\text { Center } \\
\text { Number }\end{array}$} & \multirow{2}{*}{$\begin{array}{l}\text { Atomic } \\
\text { Number }\end{array}$} & \multirow{2}{*}{$\begin{array}{l}\text { Atomic } \\
\text { Type }\end{array}$} & \multicolumn{3}{|c|}{ Coordinates (Angstroms) } \\
\hline & & & $\mathrm{X}$ & $\mathrm{Y}$ & Z \\
\hline 1 & 42 & 0 & 1.346696 & -0.331252 & 0.447216 \\
\hline 2 & 16 & 0 & 0.885926 & -2.181671 & -1.045748 \\
\hline 3 & 15 & 0 & -0.909041 & 0.141412 & 0.080148 \\
\hline 4 & 6 & 0 & -0.853222 & -2.156545 & -1.514790 \\
\hline 5 & 6 & 0 & -1.673064 & -1.116355 & -1.056987 \\
\hline 6 & 6 & 0 & -3.017165 & -1.113177 & -1.468045 \\
\hline 7 & 6 & 0 & -3.521411 & -2.114432 & -2.293406 \\
\hline 8 & 6 & 0 & -2.689790 & -3.150032 & -2.729420 \\
\hline 9 & 6 & 0 & -1.351753 & -3.168262 & -2.341358 \\
\hline 10 & 6 & 0 & -2.201378 & 0.151570 & 1.433341 \\
\hline 11 & 6 & 0 & -1.897945 & -0.463921 & 2.655186 \\
\hline 12 & 6 & 0 & -2.846533 & -0.567577 & 3.673518 \\
\hline 13 & 6 & 0 & -4.125895 & -0.045526 & 3.490043 \\
\hline 14 & 6 & 0 & -4.439479 & 0.594136 & 2.290254 \\
\hline 15 & 6 & 0 & -3.486647 & 0.695036 & 1.278096 \\
\hline 16 & 6 & 0 & -1.462944 & 1.809644 & -2.146308 \\
\hline 17 & 6 & 0 & -1.387444 & 1.711539 & -0.751136 \\
\hline 18 & 6 & 0 & -1.501660 & 2.887671 & 0.006608 \\
\hline 19 & 6 & 0 & -1.688341 & 4.122352 & -0.611047 \\
\hline 20 & 6 & 0 & -1.767607 & 4.204635 & -2.002820 \\
\hline 21 & 6 & 0 & -1.656393 & 3.043454 & -2.767254 \\
\hline 22 & 6 & 0 & 2.378843 & 1.491992 & -0.641736 \\
\hline 23 & 6 & 0 & 3.084535 & 0.309076 & -1.004041 \\
\hline 24 & 6 & 0 & 3.639046 & -0.289269 & 0.174840 \\
\hline 25 & 6 & 0 & 3.255618 & 0.529932 & 1.291738 \\
\hline 26 & 6 & 0 & 2.465593 & 1.629549 & 0.782095 \\
\hline 27 & 6 & 0 & 3.268423 & -0.184429 & -2.398246 \\
\hline 28 & 6 & 0 & 4.547857 & -1.470609 & 0.221301 \\
\hline 29 & 6 & 0 & 3.742022 & 0.380528 & 2.692997 \\
\hline 30 & 6 & 0 & 1.993558 & 2.805413 & 1.567367 \\
\hline 31 & 6 & 0 & 1.816171 & 2.490886 & -1.590376 \\
\hline 32 & 1 & 0 & -3.678805 & -0.303873 & -1.149253 \\
\hline 33 & 1 & 0 & -4.568788 & -2.086463 & -2.603664 \\
\hline 34 & 1 & 0 & -3.082839 & -3.936777 & -3.377900 \\
\hline 35 & 1 & 0 & -0.680752 & -3.960828 & -2.685891 \\
\hline 36 & 1 & 0 & -0.873432 & -0.823208 & 2.806406 \\
\hline 37 & 1 & 0 & -2.579416 & -1.039837 & 4.622942 \\
\hline 38 & 1 & 0 & -4.871737 & -0.119780 & 4.285154 \\
\hline
\end{tabular}




\begin{tabular}{|c|c|c|c|c|c|}
\hline 39 & 1 & 0 & -5.432951 & 1.026294 & 2.144987 \\
\hline 40 & 1 & 0 & -3.743810 & 1.228842 & 0.358741 \\
\hline 41 & 1 & 0 & -1.357786 & 0.907239 & -2.757656 \\
\hline 42 & 1 & 0 & -1.442729 & 2.830727 & 1.098958 \\
\hline 43 & 1 & 0 & -1.774933 & 5.026730 & -0.003077 \\
\hline 44 & 1 & 0 & -1.915738 & 5.171767 & -2.488968 \\
\hline 45 & 1 & 0 & -1.717767 & 3.098486 & -3.857270 \\
\hline 46 & 1 & 0 & 3.452937 & -1.266758 & -2.435932 \\
\hline 47 & 1 & 0 & 2.388165 & 0.016755 & -3.025326 \\
\hline 48 & 1 & 0 & 4.129059 & 0.306947 & -2.881797 \\
\hline 49 & 1 & 0 & 4.471855 & -2.005971 & 1.178133 \\
\hline 50 & 1 & 0 & 4.319778 & -2.198782 & -0.570437 \\
\hline 51 & 1 & 0 & 5.606393 & -1.183053 & 0.098259 \\
\hline 52 & 1 & 0 & 3.034135 & 0.809645 & 3.414422 \\
\hline 53 & 1 & 0 & 3.881682 & -0.674043 & 2.969051 \\
\hline 54 & 1 & 0 & 4.712009 & 0.885284 & 2.844901 \\
\hline 55 & 1 & 0 & 1.094472 & 3.254624 & 1.122277 \\
\hline 56 & 1 & 0 & 1.740604 & 2.532331 & 2.600620 \\
\hline 57 & 1 & 0 & 2.760439 & 3.598113 & 1.614364 \\
\hline 58 & 1 & 0 & 1.401818 & 2.020909 & -2.493326 \\
\hline 59 & 1 & 0 & 1.013965 & 3.091236 & -1.140108 \\
\hline 60 & 1 & 0 & 2.594899 & 3.198043 & -1.925162 \\
\hline 61 & 5 & 0 & 0.948500 & -2.699971 & 0.779300 \\
\hline 62 & 1 & 0 & 0.787107 & 0.095267 & 2.026119 \\
\hline 63 & 1 & 0 & 1.308681 & -1.782067 & 1.605053 \\
\hline 64 & 1 & 0 & 1.695209 & -3.639753 & 0.959417 \\
\hline 65 & 7 & 0 & -0.522664 & -3.170589 & 1.269338 \\
\hline 66 & 1 & 0 & -0.823094 & -4.052859 & 0.849108 \\
\hline 67 & 1 & 0 & -0.552364 & -3.286783 & 2.283324 \\
\hline 68 & 1 & 0 & -1.218545 & -2.449983 & 1.035863 \\
\hline
\end{tabular}

\section{E-Int5'(triplet)opt $=\mathbf{- 2 1 0 7 . 3 0 9 2 1 7 1}$ hartree}

\begin{tabular}{cccccc}
$\begin{array}{l}\text { Center } \\
\text { Number }\end{array}$ & $\begin{array}{c}\text { Atomic } \\
\text { Number }\end{array}$ & $\begin{array}{r}\text { Atomic } \\
\text { Type }\end{array}$ & \multicolumn{4}{c}{ Coordinates (Angstroms) } \\
- \hdashline-1 & $\mathrm{X}$ & $\mathrm{Y}$ & $\mathrm{Z}$ \\
\hline 1 & 42 & 0 & 1.199233 & 0.121899 & -0.036042 \\
2 & 16 & 0 & 0.738109 & -0.611828 & -2.354518 \\
3 & 15 & 0 & -1.245268 & -0.183996 & 0.092222 \\
4 & 6 & 0 & -0.899879 & -1.207422 & -2.436323 \\
5 & 6 & 0 & -1.822198 & -1.068559 & -1.371880 \\
6 & 6 & 0 & -3.158064 & -1.464254 & -1.538619 \\
7 & 6 & 0 & -3.584120 & -2.070291 & -2.714456 \\
8 & 6 & 0 & -2.663350 & -2.254869 & -3.753370
\end{tabular}


16

17

18

19

20

21

22

23

24

25

26

27

28

29

30

31

32

33

34

35

36

37

38

39

40

41

42

43

44

45

46

47

48

49

50

51

52
$-1.351492$

$-1.801123$

$-1.343607$

$-1.662104$

$-2.431636$

$-2.874536$

$-2.565965$

$-2.345501$

$-2.345839$

$-3.118602$

$-3.860568$

$-3.843323$

$-3.086699$

2.874061

3.250374

2.223589

1.227215

1.624729

4.509971

2.261922

0.102623

0.971859

3.655975

$-3.878812$

$-4.623312$

$-2.981101$

$-0.646046$

$-0.710263$

$-1.297640$

$-2.676337$

$-3.465596$

$-2.908953$

$-1.750317$

$-3.148957$

$-4.460244$

$-4.424737$

$-3.075739$

4.319477

4.977209

5.240619

1.296047

2.512074

3.013922

$-0.647824$
$-1.821995$

$-1.039117$

$-0.512474$

$-1.119321$

$-2.284027$

$-2.828676$

$-2.210481$

2.016097

1.284017

1.747044

2.923864

3.649617

3.187588

1.663064

1.210096

1.574492

2.316731

2.364595

0.519358

1.333943

3.075506

3.116592

1.545712

$-1.272794$

$-2.385168$

$-2.730749$

$-1.945102$

0.381450

$-0.689242$

$-2.770260$

$-3.747597$

$-2.662761$

1.658311

1.187517

3.271491

4.570085

3.743816

$-0.243753$

0.026133

1.232485

1.555949

0.289192

1.966260

3.387132
$-3.623398$

1.603678

2.822609

4.033354

4.046746

2.843701

1.630607

$-1.158457$

0.039843

1.110810

0.991879

$-0.197141$

$-1.276785$

$-0.732260$

0.562639

1.488127

0.763375

$-0.610409$

0.938314

2.959362

1.381970

$-1.718605$

$-1.994624$

$-0.737416$

$-2.828504$

$-4.684929$

$-4.448534$

2.812803

4.969414

4.993893

2.843630

0.697669

$-2.005878$

2.049329

1.836769

$-0.287721$

$-2.217532$

1.707558

0.075824

1.353964

3.434191

3.202019

3.462099

0.644395 


\begin{tabular}{|c|c|c|c|c|c|}
\hline 53 & 1 & 0 & -0.429068 & 2.502433 & 2.155977 \\
\hline 54 & 1 & 0 & 0.477452 & 3.990884 & 1.868959 \\
\hline 55 & 1 & 0 & 0.864053 & 2.499232 & -2.623610 \\
\hline 56 & 1 & 0 & -0.028399 & 3.473865 & -1.441373 \\
\hline 57 & 1 & 0 & 1.565488 & 4.002391 & -2.000043 \\
\hline 58 & 1 & 0 & 4.428528 & 0.769162 & -1.929967 \\
\hline 59 & 1 & 0 & 3.012779 & 1.289996 & -2.849701 \\
\hline 60 & 1 & 0 & 4.156697 & 2.496575 & -2.242133 \\
\hline 61 & 5 & 0 & 3.244036 & -2.174550 & -0.645157 \\
\hline 62 & 1 & 0 & 0.789289 & -1.942753 & 1.747901 \\
\hline 63 & 1 & 0 & 2.786338 & -3.050443 & -1.365205 \\
\hline 64 & 6 & 0 & 1.063108 & -1.995582 & 0.673214 \\
\hline 65 & 7 & 0 & 2.329284 & -1.555942 & 0.378521 \\
\hline 66 & 6 & 0 & 0.435963 & -3.175348 & -0.021813 \\
\hline 67 & 1 & 0 & -0.628003 & -3.283781 & 0.233385 \\
\hline 68 & 1 & 0 & 0.524663 & -3.116911 & -1.113966 \\
\hline 69 & 1 & 0 & 0.925992 & -4.117352 & 0.287096 \\
\hline 70 & 1 & 0 & 3.931379 & -1.374869 & -1.266141 \\
\hline 71 & 7 & 0 & 4.358186 & -3.008791 & 0.293325 \\
\hline 72 & 1 & 0 & 5.067986 & -3.488146 & -0.260365 \\
\hline 73 & 1 & 0 & 4.823683 & -2.366254 & 0.935366 \\
\hline 74 & 1 & 0 & 3.879979 & -3.706490 & 0.863866 \\
\hline
\end{tabular}

\section{E-TS3'(singlet)opt $=\mathbf{- 2 1 0 7 . 2 9 1 4 5 3 5}$ hartree}

\begin{tabular}{rrrrrr}
$\begin{array}{l}\text { Center } \\
\text { Number }\end{array}$ & $\begin{array}{c}\text { Atomic } \\
\text { Number }\end{array}$ & $\begin{array}{r}\text { Atomic } \\
\text { Type }\end{array}$ & \multicolumn{2}{c}{ Coordinates (Angstroms) } \\
- \hdashline 1 & 42 & 0 & 1.220681 & -0.091012 & 0.204251 \\
2 & 16 & 0 & 0.506627 & -0.768322 & 2.299685 \\
3 & 15 & 0 & -1.136520 & 0.214786 & -0.076252 \\
4 & 6 & 0 & -1.154379 & -0.229376 & 2.615717 \\
5 & 6 & 0 & -1.944012 & 0.259664 & 1.564875 \\
6 & 6 & 0 & -3.290192 & 0.564940 & 1.797777 \\
7 & 6 & 0 & -3.825103 & 0.478187 & 3.081320 \\
8 & 6 & 0 & -3.018224 & 0.044943 & 4.137185 \\
9 & 6 & 0 & -1.697920 & -0.326941 & 3.905208 \\
10 & 6 & 0 & -1.699884 & 1.689487 & -1.008087 \\
11 & 6 & 0 & -1.194876 & 1.843530 & -2.309606 \\
12 & 6 & 0 & -1.490198 & 2.970402 & -3.070613 \\
13 & 6 & 0 & -2.280421 & 3.987768 & -2.533503 \\
14 & 6 & 0 & -2.763725 & 3.863143 & -1.233858 \\
15 & 6 & 0 & -2.478743 & 2.724314 & -0.477673 \\
16 & 6 & 0 & -2.533891 & -2.211686 & -0.006919
\end{tabular}




\begin{tabular}{|c|c|}
\hline 17 & 6 \\
\hline 18 & 6 \\
\hline 19 & 6 \\
\hline 20 & 6 \\
\hline 21 & 6 \\
\hline 22 & 6 \\
\hline 23 & 6 \\
\hline 24 & 6 \\
\hline 25 & 6 \\
\hline 26 & 6 \\
\hline 27 & 6 \\
\hline 28 & 6 \\
\hline 29 & 6 \\
\hline 30 & 6 \\
\hline 31 & 6 \\
\hline 32 & 1 \\
\hline 33 & 1 \\
\hline 34 & 1 \\
\hline 35 & 1 \\
\hline 36 & 1 \\
\hline 37 & 1 \\
\hline 38 & 1 \\
\hline 39 & 1 \\
\hline 40 & 1 \\
\hline 41 & 1 \\
\hline 42 & 1 \\
\hline 43 & 1 \\
\hline 44 & 1 \\
\hline 45 & 1 \\
\hline 46 & 1 \\
\hline 47 & 1 \\
\hline 48 & 1 \\
\hline 49 & 1 \\
\hline 50 & 1 \\
\hline 51 & 1 \\
\hline 52 & 1 \\
\hline 53 & 1 \\
\hline 54 & 1 \\
\hline 55 & 1 \\
\hline 56 & 1 \\
\hline 57 & 1 \\
\hline 58 & 1 \\
\hline 59 & 1 \\
\hline 60 & 1 \\
\hline
\end{tabular}

\begin{tabular}{rrr}
-2.198448 & -1.110765 & -0.812218 \\
-2.672742 & -1.090418 & -2.130872 \\
-3.438947 & -2.142794 & -2.634163 \\
-3.754430 & -3.232433 & -1.825397 \\
-3.302156 & -3.259344 & -0.505941 \\
2.474776 & -2.104823 & 0.108442 \\
3.351939 & -1.093708 & -0.379759 \\
2.775363 & -0.541461 & -1.564866 \\
1.548811 & -1.215492 & -1.834963 \\
1.365758 & -2.195381 & -0.793684 \\
4.702466 & -0.800132 & 0.167216 \\
3.354763 & 0.533515 & -2.419169 \\
0.802481 & -1.159645 & -3.122697 \\
0.373370 & -3.307347 & -0.799826 \\
2.737660 & -3.015957 & 1.254539 \\
-3.937123 & 0.848915 & 0.962761 \\
-4.872698 & 0.732765 & 3.256112 \\
-3.431575 & -0.026648 & 5.146443 \\
-1.075945 & -0.707433 & 4.719589 \\
-0.533782 & 1.073228 & -2.717067 \\
-1.088659 & 3.062174 & -4.082766 \\
-2.506125 & 4.879611 & -3.122500 \\
-3.365786 & 4.661982 & -0.794390 \\
-2.852500 & 2.665051 & 0.545663 \\
-2.184977 & -2.251880 & 1.028828 \\
-2.460897 & -0.237283 & -2.779071 \\
-3.801104 & -2.100392 & -3.664299 \\
-4.359418 & -4.053009 & -2.217891 \\
-3.550537 & -4.103138 & 0.142372 \\
5.066031 & 0.192008 & -0.126340 \\
4.733429 & -0.849174 & 1.262541 \\
5.433911 & -1.531488 & -0.215447 \\
2.595336 & 1.271039 & -2.720687 \\
4.154380 & 1.085590 & -1.908908 \\
3.785438 & 0.120932 & -3.346291 \\
-0.278413 & -1.307729 & -2.996041 \\
0.957118 & -0.206917 & -3.647720 \\
1.149110 & -1.953957 & -3.806234 \\
-0.080237 & -3.481405 & 0.186506 \\
-0.442351 & -3.135008 & -1.512971 \\
3.293307 & -3.912775 & 0.933519 \\
\hline & -4.251013 & -1.095377 \\
\hline
\end{tabular}




$\begin{array}{rrrrrr}61 & 5 & 0 & 3.319564 & 2.002028 & 1.584680 \\ 62 & 1 & 0 & 0.525041 & 2.610213 & -0.400235 \\ 63 & 1 & 0 & 2.916725 & 2.799528 & 2.406343 \\ 64 & 6 & 0 & 0.855941 & 2.102330 & 0.518069 \\ 65 & 7 & 0 & 2.182505 & 1.691313 & 0.482862 \\ 66 & 6 & 0 & 0.282873 & 2.693623 & 1.772979 \\ 67 & 1 & 0 & -0.811689 & 2.785521 & 1.717227 \\ 68 & 1 & 0 & 0.528645 & 2.097513 & 2.662701 \\ 69 & 1 & 0 & 0.681127 & 3.708110 & 1.944131 \\ 70 & 1 & 0 & 3.828390 & 1.006928 & 2.057134 \\ 71 & 7 & 0 & 4.251627 & 2.696847 & 0.482208 \\ 72 & 1 & 0 & 5.168680 & 2.290560 & 0.313378 \\ 73 & 1 & 0 & 3.171880 & 2.257816 & -0.124480 \\ 74 & 1 & 0 & 4.358822 & 3.704537 & 0.566262\end{array}$

\section{E-Int6'(singlet)opt $=\mathbf{- 2 1 0 7 . 3 3 3 4 1 2}$ hartree}

\begin{tabular}{rrrrrr}
$\begin{array}{r}\text { Center } \\
\text { Number }\end{array}$ & $\begin{array}{r}\text { Atomic } \\
\text { Number }\end{array}$ & $\begin{array}{r}\text { Atomic } \\
\text { Type }\end{array}$ & \multicolumn{2}{c}{ Coordinates (Angstroms) } \\
-1 & 42 & 0 & -1.364511 & -0.221796 & -0.182370 \\
\hline 1 & 16 & 0 & -0.565295 & -2.445438 & -0.885648 \\
3 & 15 & 0 & 1.129334 & 0.158509 & -0.068072 \\
4 & 6 & 0 & 1.095762 & -2.276671 & -1.370089 \\
5 & 6 & 0 & 1.884969 & -1.135009 & -1.084615 \\
6 & 6 & 0 & 3.255404 & -1.139629 & -1.403672 \\
7 & 6 & 0 & 3.838503 & -2.201535 & -2.080270 \\
8 & 6 & 0 & 3.046515 & -3.309167 & -2.414235 \\
9 & 6 & 0 & 1.709930 & -3.351234 & -2.055251 \\
10 & 6 & 0 & 1.571186 & 1.824838 & -0.729878 \\
11 & 6 & 0 & 0.809926 & 2.900856 & -0.248341 \\
12 & 6 & 0 & 1.053314 & 4.206077 & -0.673433 \\
13 & 6 & 0 & 2.053649 & 4.457770 & -1.611700 \\
14 & 6 & 0 & 2.799547 & 3.394815 & -2.118696 \\
15 & 6 & 0 & 2.564735 & 2.091478 & -1.680731 \\
16 & 6 & 0 & 2.808390 & -1.127456 & 1.781458 \\
17 & 6 & 0 & 2.293326 & 0.111940 & 1.366905 \\
18 & 6 & 0 & 2.702452 & 1.261468 & 2.058366 \\
19 & 6 & 0 & 3.577526 & 1.174316 & 3.140907 \\
20 & 6 & 0 & 4.068060 & -0.064448 & 3.548916 \\
21 & 6 & 0 & 3.682870 & -1.215195 & 2.860736 \\
22 & 6 & 0 & -2.445768 & -1.464643 & 1.416045 \\
23 & 6 & 0 & -3.377377 & -0.419260 & 1.126062 \\
24 & 6 & 0 & -2.767637 & 0.825412 & 1.446511
\end{tabular}


25

26

27

28

29

30

31

32

33

34

35

36

37

38

39

40

41

42

43

44

45

46

47

48

49

50

51

52

53

54

55

56

57

58

59

60

61

62

63

64

65

66

67

68
$-1.465114$

$-1.266843$

$-4.781058$

$-3.410977$

$-0.695879$

$-0.234774$

$-2.752994$

3.886016

4.900671

3.488226

1.103021

0.002174

0.452619

2.243093

3.571715

3.144409

2.521006

2.342802

3.884796

4.757172

4.069744

$-5.116220$

$-4.906562$

$-5.443564$

$-2.832558$

$-4.423190$

$-3.487253$

0.379029

$-0.826501$

$-1.047774$

0.248405

0.548749

$-0.695089$

$-3.273032$

$-1.839941$

$-3.399039$

$-3.094519$

0.285201

$-3.300510$

$-0.659109$

$-1.690606$

$-0.836422$

0.089486

$-1.651453$
0.574798

$-0.864584$

$-0.635517$

2.170845

1.566158

$-1.625060$

$-2.921135$

$-0.307551$

$-2.179217$

$-4.156410$

$-4.229073$

2.693844

5.028313

5.477042

3.578177

1.275531

$-2.037977$

2.245348

2.085192

$-0.132686$

$-2.191193$

0.149715

$-1.607467$

$-0.628761$

2.917443

2.138632

2.560581

1.354121

2.595114

1.558615

$-2.401900$

$-0.970850$

$-2.135609$

$-3.219563$

$-3.525647$

$-3.201206$

0.686460

0.898232

0.140648

0.354769

1.235043

$-0.512087$

$-1.081715$

$-1.238993$
1.975474

1.979079

0.694297

1.440432

2.777551

2.738090

1.387582

$-1.078491$

$-2.331937$

$-2.945508$

$-2.290580$

0.461237

$-0.276140$

$-1.955718$

$-2.869639$

$-2.114767$

1.249515

1.747038

3.660237

4.393833

3.163030

0.004209

0.200159

1.574977

0.872701

1.022723

2.468528

2.809556

2.414835

3.824131

2.126706

3.138843

3.600223

0.466729

1.447397

2.236345

$-1.991416$

$-2.285304$

$-3.057469$

$-2.207369$

$-1.764005$

$-3.424508$

$-3.585061$

$-3.344718$ 


$\begin{array}{llllll}69 & 1 & 0 & -1.003747 & 0.098306 & -4.328019 \\ 70 & 1 & 0 & -2.869845 & -0.533352 & -1.291670 \\ 71 & 7 & 0 & -4.193988 & 1.538147 & -1.538903 \\ 72 & 1 & 0 & -4.983684 & 1.624591 & -2.163959 \\ 73 & 1 & 0 & -1.477876 & 2.231292 & -1.741473 \\ 74 & 1 & 0 & -3.994229 & 2.431169 & -1.107810\end{array}$




\section{Characterization of hydrogenation products}

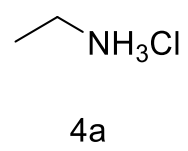

Ethylamine hydrochloride (4a). ${ }^{17}$

${ }^{1} \mathrm{H}$ NMR (500 MHz, $\left.\mathrm{D}_{2} \mathrm{O}\right) \delta 3.27-3.04\left(\mathrm{~m}, 2 \mathrm{H}, \mathrm{CH}_{2}\right), 1.34\left(\mathrm{t}, \mathrm{J}=6.6 \mathrm{~Hz}, 3 \mathrm{H}, \mathrm{CH}_{3}\right) .{ }^{13} \mathrm{C}$ NMR (126 MHz, $\left.\mathrm{D}_{2} \mathrm{O}\right) \delta 35.09,11.98$.<smiles>NCC1CCCCC1</smiles>

$4 \mathrm{~b}$

Cyclohexylmethylamine hydrochloride (4b). ${ }^{18}$

${ }^{1} \mathrm{H}$ NMR $\left(500 \mathrm{MHz}, \mathrm{D}_{2} \mathrm{O}\right) \delta 2.86\left(\mathrm{~d}, J=7.0 \mathrm{~Hz}, 2 \mathrm{H}, \mathrm{CH}_{2}\right), 1.74(\mathrm{~d}, J=11.1 \mathrm{~Hz}, 4 \mathrm{H}$, $\left.\mathrm{C}_{6} H_{11}\right), 1.66\left(\mathrm{dd}, J=9.6,2.6 \mathrm{~Hz}, 2 \mathrm{H}, \mathrm{C}_{6} H_{11}\right), 1.25\left(\mathrm{dd}, J=25.5,22.6 \mathrm{~Hz}, 3 \mathrm{H}, \mathrm{C}_{6} H_{11}\right), 1.05$ $-0.95\left(\mathrm{~m}, 2 \mathrm{H}, \mathrm{C}_{6} H_{11}\right) .{ }^{13} \mathrm{C} \mathrm{NMR}\left(126 \mathrm{MHz}, \mathrm{D}_{2} \mathrm{O}\right) \delta 45.23,35.41,29.64,25.59,25.04$.

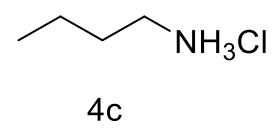

n-Butylamine hydrochloride (4c). ${ }^{19}$

${ }^{1} \mathrm{H}$ NMR (500 MHz, D $\left.2 \mathrm{O}\right) \delta 3.07-2.88\left(\mathrm{~m}, 2 \mathrm{H}, \mathrm{CH}_{3} \mathrm{CH}_{2} \mathrm{CH}_{2} \mathrm{CH}_{2}\right), 1.70-1.58(\mathrm{~m}, 2 \mathrm{H}$, $\mathrm{CH}_{3} \mathrm{CH}_{2} \mathrm{CH}_{2} \mathrm{CH}_{2}$ ), 1.40 (dt, $\left.J=15.1,7.4 \mathrm{~Hz}, 2 \mathrm{H}, \mathrm{CH}_{3} \mathrm{CH}_{2} \mathrm{CH}_{2} \mathrm{CH}_{2}\right), 0.93$ (t, $J=7.4 \mathrm{~Hz}$, $\left.3 \mathrm{H}, \mathrm{CH}_{3}\right) .{ }^{13} \mathrm{C} \mathrm{NMR}\left(126 \mathrm{MHz}, \mathrm{D}_{2} \mathrm{O}\right) \delta 39.26,28.74,18.97,12.73$.

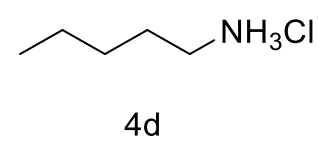

Pentamine hydrochloride (4d).

${ }^{1} \mathrm{H}$ NMR (500 MHz, D $\left.2 \mathrm{O}\right) \delta 3.04-2.92\left(\mathrm{~m}, 2 \mathrm{H}, \mathrm{CH}_{3} \mathrm{CH}_{2} \mathrm{CH}_{2} \mathrm{CH}_{2} \mathrm{CH}_{2}\right), 1.73-1.59(\mathrm{~m}$, $2 \mathrm{H}, \mathrm{CH}_{3} \mathrm{CH}_{2} \mathrm{CH}_{2} \mathrm{CH}_{2} \mathrm{CH}_{2}$ ), 1.35 (dd, $\left.J=8.8,5.5 \mathrm{~Hz}, 4 \mathrm{H}, \mathrm{CH}_{3} \mathrm{CH}_{2} \mathrm{CH}_{2} \mathrm{CH}_{2} \mathrm{CH}_{2}\right), 0.93-$ $0.82\left(\mathrm{~m}, 3 \mathrm{H}, \mathrm{CH}_{3} \mathrm{CH}_{2} \mathrm{CH}_{2} \mathrm{CH}_{2} \mathrm{CH}_{2}\right) .{ }^{13} \mathrm{C} \mathrm{NMR}\left(126 \mathrm{MHz}, \mathrm{D}_{2} \mathrm{O}\right) \delta$ 39.52, 27.70, 26.36, 21.44, 13.06 . 


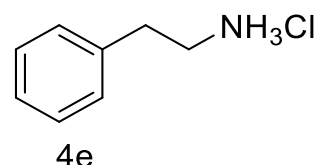

B-Phenylethylamine hydrochloride (4e). ${ }^{18}$

${ }^{1} \mathrm{H}$ NMR (500 MHz, D $\left.2 \mathrm{O}\right) \delta 7.43(\mathrm{dd}, J=10.1,4.5 \mathrm{~Hz}, 2 \mathrm{H}, \mathrm{ArH}), 7.38-7.31(\mathrm{~m}, 3 \mathrm{H}$, $\operatorname{Ar} H$ ), 3.28 (t, $J=7.3 \mathrm{~Hz}, 2 \mathrm{H} \mathrm{CH} \mathrm{NH}_{3} \mathrm{Cl}$ ), 3.00 (t, $\left.J=7.3 \mathrm{~Hz}, 2 \mathrm{H}, \mathrm{CH}_{2} \mathrm{CH}_{2} \mathrm{NH}_{3} \mathrm{Cl}\right) .{ }^{13} \mathrm{C}$ NMR (126 MHz, D $2 \mathrm{O}) \delta 136.64,129.08,128.90,127.36,40.60,32.73$.

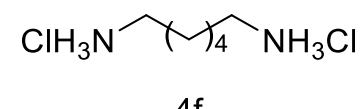

$4 f$

Hexamethylenediamine dihydrochloride (4f). ${ }^{18}$

${ }^{1} \mathrm{H}$ NMR (500 MHz, D $\left.2 \mathrm{O}\right) \delta 3.11-2.86\left(\mathrm{~m}, 4 \mathrm{H}, \mathrm{NH}_{3} \mathrm{ClCH}_{2} \mathrm{CH}_{2} \mathrm{CH}_{2} \mathrm{CH}_{2} \mathrm{CH}_{2} \mathrm{CH}_{2} \mathrm{NH}_{3} \mathrm{Cl}\right)$, $1.74-1.58$ (m, 4H, $\mathrm{NH}_{3} \mathrm{ClCH}_{2} \mathrm{CH}_{2} \mathrm{CH}_{2} \mathrm{CH}_{2} \mathrm{CH}_{2} \mathrm{CH}_{2} \mathrm{NH}_{3} \mathrm{Cl}$ ), 1.39 (dd, $J=8.8,5.5 \mathrm{~Hz}$, $\left.4 \mathrm{H}, \mathrm{NH}_{3} \mathrm{ClCH}_{2} \mathrm{CH}_{2} \mathrm{CH}_{2} \mathrm{CH}_{2} \mathrm{CH}_{2} \mathrm{CH}_{2} \mathrm{NH}_{3} \mathrm{Cl}\right) .{ }^{13} \mathrm{C} \mathrm{NMR}\left(126 \mathrm{MHz}, \mathrm{D}_{2} \mathrm{O}\right) \delta 39.33,26.46$, 25.07 .

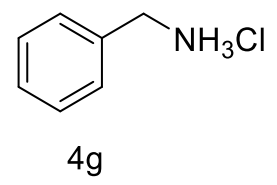

Benzylamine hydrochloride (4g) ${ }^{18}$

${ }^{1} \mathrm{H}$ NMR (500 MHz, D $\left.2 \mathrm{O}\right) \delta 7.51-7.45(\mathrm{~m}, 5 \mathrm{H}, \mathrm{Ar} H), 4.19\left(\mathrm{~s}, 2 \mathrm{H}, \mathrm{CH}_{2}\right) .{ }^{13} \mathrm{C}$ NMR $(126$ $\left.\mathrm{MHz}, \mathrm{D}_{2} \mathrm{O}\right) \delta 132.66,129.30,128.92,43.21$.

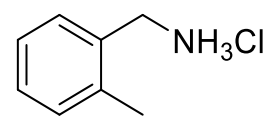

$4 \mathrm{~h}$

2-Methylbenzylamine hydrochloride (4h). ${ }^{18}$

${ }^{1} \mathrm{H}$ NMR (500 MHz, D $\left.2 \mathrm{O}\right) \delta 7.44-7.23(\mathrm{~m}, 4 \mathrm{H}, \mathrm{ArH}), 4.24\left(\mathrm{~s}, 2 \mathrm{H}, \mathrm{CH}_{2}\right), 2.39(\mathrm{~s}, 3 \mathrm{H}$, $\left.\mathrm{CH}_{3}\right) .{ }^{13} \mathrm{C}$ NMR $\left(126 \mathrm{MHz}, \mathrm{D}_{2} \mathrm{O}\right) \delta 137.18,130.90,129.37,129.03,126.63,40.44,18.01$. 


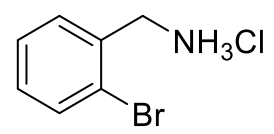

$4 \mathrm{i}$

2-Bromobenzylamine hydrochloride (4i).

${ }^{1} \mathrm{H}$ NMR (500 MHz, D $\left.2 \mathrm{O}\right) \delta 7.75(\mathrm{~d}, J=7.9 \mathrm{~Hz}, 1 \mathrm{H}, \mathrm{Ar} H), 7.54(\mathrm{~d}, J=7.1 \mathrm{~Hz}, 1 \mathrm{H}, \mathrm{Ar} H)$, $7.48(\mathrm{t}, J=7.4 \mathrm{~Hz}, 1 \mathrm{H}, \mathrm{Ar} H), 7.39(\mathrm{t}, J=7.6 \mathrm{~Hz}, 1 \mathrm{H}, \mathrm{Ar} H), 4.35\left(\mathrm{~s}, 2 \mathrm{H}, \mathrm{CH}_{2}\right) .{ }^{13} \mathrm{C} \mathrm{NMR}$ (126 MHz, $\left.\mathrm{D}_{2} \mathrm{O}\right) \delta 133.33,131.91,131.28$ (d, $\left.J=12.8 \mathrm{~Hz}\right), 128.50,123.86,43.31$.

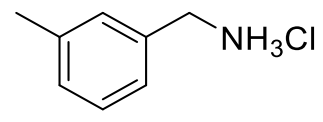

$4 \mathrm{j}$

3-Methylbenzylamine hydrochloride (4j).

${ }^{1} \mathrm{H}$ NMR (500 MHz, D $\left.2 \mathrm{O}\right) \delta 7.46-7.21(\mathrm{~m}, 4 \mathrm{H}, \mathrm{ArH}), 4.16\left(\mathrm{~s}, 2 \mathrm{H}, \mathrm{CH}_{2}\right), 2.38(\mathrm{~s}, 3 \mathrm{H}$, $\left.\mathrm{CH}_{3}\right) .{ }^{13} \mathrm{C} \mathrm{NMR}\left(126 \mathrm{MHz}, \mathrm{D}_{2} \mathrm{O}\right) \delta 139.52,132.69,129.87,129.43,129.23,125.78,43.15$, 20.46 .

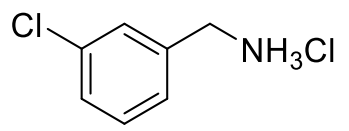

$4 \mathrm{k}$

3-Chlorobenzylamine hydrochloride (4k).

${ }^{1} \mathrm{H}$ NMR (500 MHz, D $\left.2 \mathrm{O}\right) \delta 7.54-7.38(\mathrm{~m}, 4 \mathrm{H}, \mathrm{Ar} H), 4.20\left(\mathrm{~s}, 2 \mathrm{H}, \mathrm{CH}_{2}\right) .{ }^{13} \mathrm{C}$ NMR $(126$ $\left.\mathrm{MHz}, \mathrm{D}_{2} \mathrm{O}\right) \delta 134.51,134.16,130.72,129.25,128.78,127.24,42.56$.

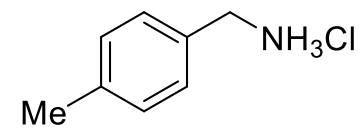

41

4-Methylbenzylamine hydrochloride (4I). ${ }^{18}$

${ }^{1} \mathrm{H}$ NMR (500 MHz, D $\left.2 \mathrm{O}\right) \delta 7.38(\mathrm{~d}, \mathrm{~J}=8.1 \mathrm{~Hz}, 2 \mathrm{H}, \operatorname{Ar} H), 7.33(\mathrm{~d}, J=8.1 \mathrm{~Hz}, 2 \mathrm{H}, \operatorname{Ar} H)$, 4.16 (s, 2H, $\left.\mathrm{CH}_{2}\right), 2.37$ (s, 3H, $\left.\mathrm{CH}_{3}\right) .{ }^{13} \mathrm{C} \mathrm{NMR}\left(126 \mathrm{MHz}, \mathrm{D}_{2} \mathrm{O}\right) \delta 139.68,129.84,129.65$, $128.97,42.96,20.36$. 


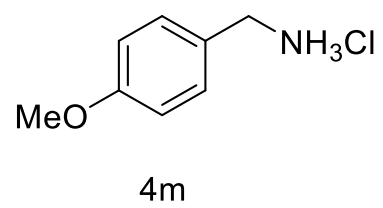

4-Methoxybenzylamine hydrochloride $(\mathbf{4 m}){ }^{18}$

${ }^{1} \mathrm{H}$ NMR (500 MHz, D $\left.2 \mathrm{O}\right) \delta 7.45(\mathrm{~d}, J=8.5 \mathrm{~Hz}, 2 \mathrm{H}, \mathrm{Ar} H), 7.07(\mathrm{~d}, J=8.4 \mathrm{~Hz}, 2 \mathrm{H}, \mathrm{Ar} H)$,

4.17 (s, 2H, $\left.\mathrm{CH}_{2}\right), 3.87$ (s, 3H, $\left.\mathrm{OCH}_{3}\right) .{ }^{13} \mathrm{C} \mathrm{NMR}\left(126 \mathrm{MHz}, \mathrm{D}_{2} \mathrm{O}\right) \delta 159.45,130.71$, 125.24, 114.68, 55.57, 42.71 .<smiles>NCc1ccc(C(F)(F)F)cc1</smiles>

4-Trifluoromethylamine hydrochloride (4n).

${ }^{1} \mathrm{H}$ NMR (500 MHz, D $\left.2 \mathrm{O}\right) \delta 7.81(\mathrm{~d}, J=8.1 \mathrm{~Hz}, 2 \mathrm{H}, \operatorname{Ar} H), 7.64(\mathrm{~d}, J=8.1 \mathrm{~Hz}, 2 \mathrm{H}, \operatorname{Ar} H)$, $4.29\left(\mathrm{~s}, 2 \mathrm{H}, \mathrm{CH}_{2}\right) .{ }^{13} \mathrm{C} \mathrm{NMR}\left(126 \mathrm{MHz}, \mathrm{D}_{2} \mathrm{O}\right) \delta 136.66,130.45$ (q, $\left.J_{\mathrm{C}-\mathrm{F}}=32 \mathrm{~Hz}\right), 129.33$, $126.10\left(\mathrm{q}, J_{\mathrm{C}-\mathrm{F}}=3.8 \mathrm{~Hz}\right), 125.10,122.94,120.78,42.62$.

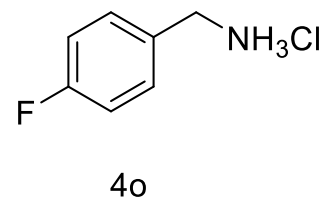

4-Fluorobenzylamine hydrochloride (40). ${ }^{18}$

${ }^{1} \mathrm{H}$ NMR (500 MHz, $\left.\mathrm{D}_{2} \mathrm{O}\right) \delta 7.52(\mathrm{dd}, J=8.5,5.4 \mathrm{~Hz}, 2 \mathrm{H}, \mathrm{Ar} H), 7.24(\mathrm{t}, J=8.8 \mathrm{~Hz}, 2 \mathrm{H}$, $\mathrm{Ar} H), 4.22\left(\mathrm{~s}, 2 \mathrm{H}, \mathrm{CH}_{2}\right) .{ }^{13} \mathrm{C} \mathrm{NMR}\left(126 \mathrm{MHz}, \mathrm{D}_{2} \mathrm{O}\right) \delta 42.49,116.13\left(\mathrm{~d}, J_{\mathrm{C}-\mathrm{F}}=21.4 \mathrm{~Hz}\right)$, $128.67\left(\mathrm{~d}, J_{\mathrm{C}-\mathrm{F}}=2.5 \mathrm{~Hz}\right), 131.21\left(\mathrm{~d}, J_{\mathrm{C}-\mathrm{F}}=8.8 \mathrm{~Hz}\right), 163.89\left(\mathrm{~d}, J_{\mathrm{C}-\mathrm{F}}=245.7 \mathrm{~Hz}\right)$.<smiles>NCc1ccc(Cl)cc1</smiles>

\section{4-Chlorobenzylamine hydrochloride (4p). ${ }^{18}$}

${ }^{1} \mathrm{H}$ NMR $\left(500 \mathrm{MHz}, \mathrm{D}_{2} \mathrm{O}\right) \delta 7.45(\mathrm{dd}, J=23.4,7.5 \mathrm{~Hz}, 4 \mathrm{H}, \mathrm{ArH}), 4.18\left(\mathrm{~s}, 2 \mathrm{H}, \mathrm{CH}_{2}\right) .{ }^{13} \mathrm{C}$ NMR (126 MHz, D $2 \mathrm{O}) \delta 134.53,131.21,130.51,129.19,42.49$. 
<smiles>COC(=O)c1ccc(CN)cc1</smiles>

$4 q$

4-(Aminomethyl)benzoate hydrochloride (4q) ${ }^{18}$

${ }^{1} \mathrm{H}$ NMR (500 MHz, D $\left.2 \mathrm{O}\right) \delta 8.23$ - $7.90(\mathrm{~m}, 2 \mathrm{H}, \mathrm{ArH}), 7.75-7.34$ (m, 2H, ArH), $4.28(\mathrm{~d}$, $\left.J=6.0 \mathrm{~Hz}, 2 \mathrm{H}, \mathrm{CH}_{2}\right), 3.93\left(\mathrm{dd}, J=6.6,3.9 \mathrm{~Hz}, 3 \mathrm{H}, \mathrm{COOCH}_{3}\right) .{ }^{13} \mathrm{C} \mathrm{NMR}\left(126 \mathrm{MHz}, \mathrm{D}_{2} \mathrm{O}\right)$ $\delta 168.79,137.96,130.12,128.95,52.81,42.70$.

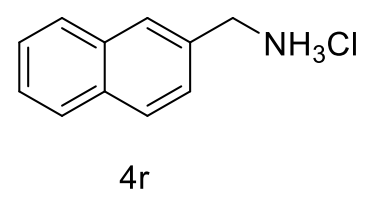

Naphthalen-2-ylmethanammonium chloride (4r).

${ }^{1} \mathrm{H}$ NMR (500 MHz, D $\left.2 \mathrm{O}\right) \delta 7.93(\mathrm{ddd}, J=9.8,9.1,4.7 \mathrm{~Hz}, 4 \mathrm{H}, \mathrm{Ar} H), 7.60-7.56(\mathrm{~m}, 2 \mathrm{H}$, $\operatorname{Ar} H), 7.50(\mathrm{dd}, J=8.5,1.8 \mathrm{~Hz}, 1 \mathrm{H}, \mathrm{Ar} H), 4.29\left(\mathrm{~s}, 2 \mathrm{H}, \mathrm{CH}_{2}\right) .{ }^{13} \mathrm{C} \mathrm{NMR}\left(126 \mathrm{MHz}, \mathrm{D}_{2} \mathrm{O}\right)$ $\delta 132.91(\mathrm{~d}, J=9.6 \mathrm{~Hz}), 130.15,128.95,128.18,127.97,127.73,127.00$ (d, $J=19.8 \mathrm{~Hz})$, $125.94,43.21$.

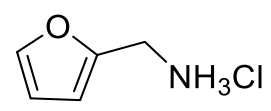

$4 s$

Furan-2-ylmethanammonium hydrochloride (4s).

${ }^{1} \mathrm{H}$ NMR $\left(500 \mathrm{MHz}, \mathrm{D}_{2} \mathrm{O}\right) \delta 7.60(\mathrm{~d}, J=1.7 \mathrm{~Hz}, 1 \mathrm{H}), 6.59(\mathrm{~d}, J=3.3 \mathrm{~Hz}, 1 \mathrm{H}), 6.51$ (dd, $J=3.1,2.0 \mathrm{~Hz}, 1 \mathrm{H}), 4.25\left(\mathrm{~s}, 2 \mathrm{H}, \mathrm{CH}_{2}\right) .{ }^{13} \mathrm{C} \mathrm{NMR}\left(126 \mathrm{MHz}, \mathrm{D}_{2} \mathrm{O}\right) \delta 146.19,144.43$, 110.94, 35.72.

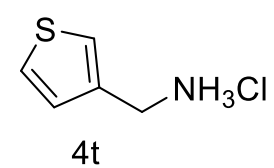

Thiophen-3-ylmethanammonium hydrochloride $(4 \mathrm{t}){ }^{20}$

${ }^{1} \mathrm{H}$ NMR $\left(500 \mathrm{MHz}, \mathrm{D}_{2} \mathrm{O}\right) \delta 7.56$ (s, 2H), $7.22(\mathrm{~s}, 1 \mathrm{H}), 4.24\left(\mathrm{~s}, 2 \mathrm{H}, \mathrm{CH}_{2}\right) .{ }^{13} \mathrm{C}$ NMR $(126$ $\left.\mathrm{MHz}, \mathrm{D}_{2} \mathrm{O}\right) \delta 133.01,127.63,127.37,125.88,37.83$. 
8. Characterization of molybdenum compounds

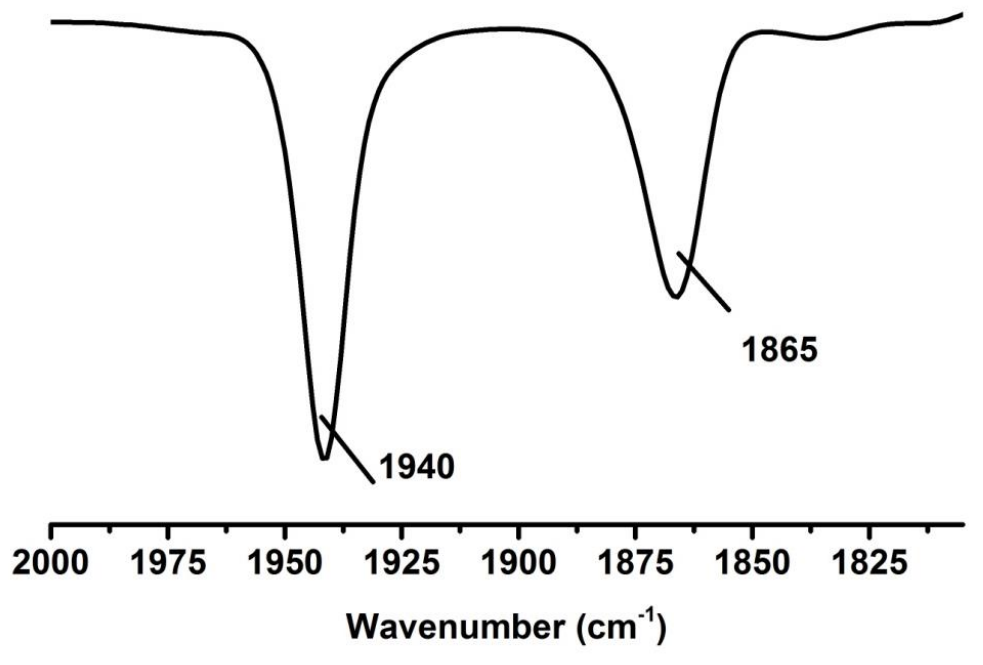

Figure S24. IR spectrum ( $v_{\mathrm{CO}}$ region) of $\mathbf{1}(\mathrm{CO})_{2}$ in THF. 


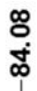

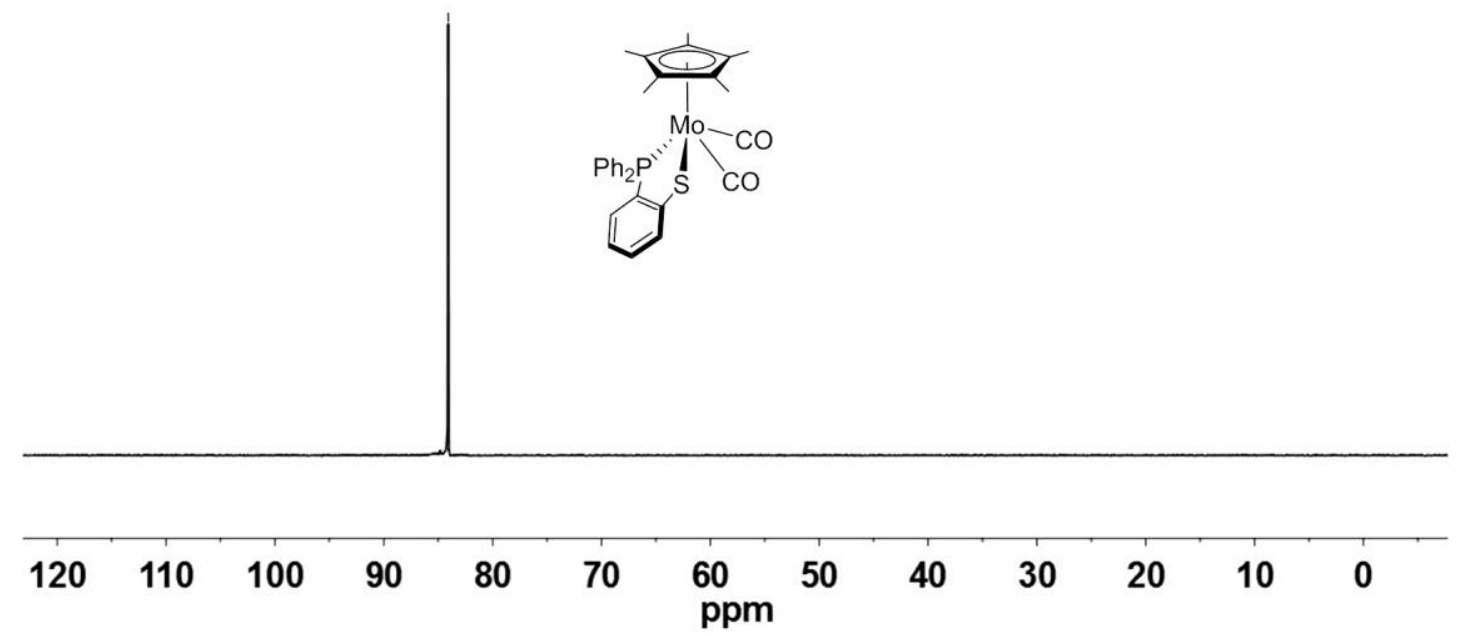

Figure S25. ${ }^{31} \mathrm{P}$ NMR spectrum of $\mathbf{1}(\mathrm{CO})_{2}$ in $\mathrm{CDCl}_{3}$. 


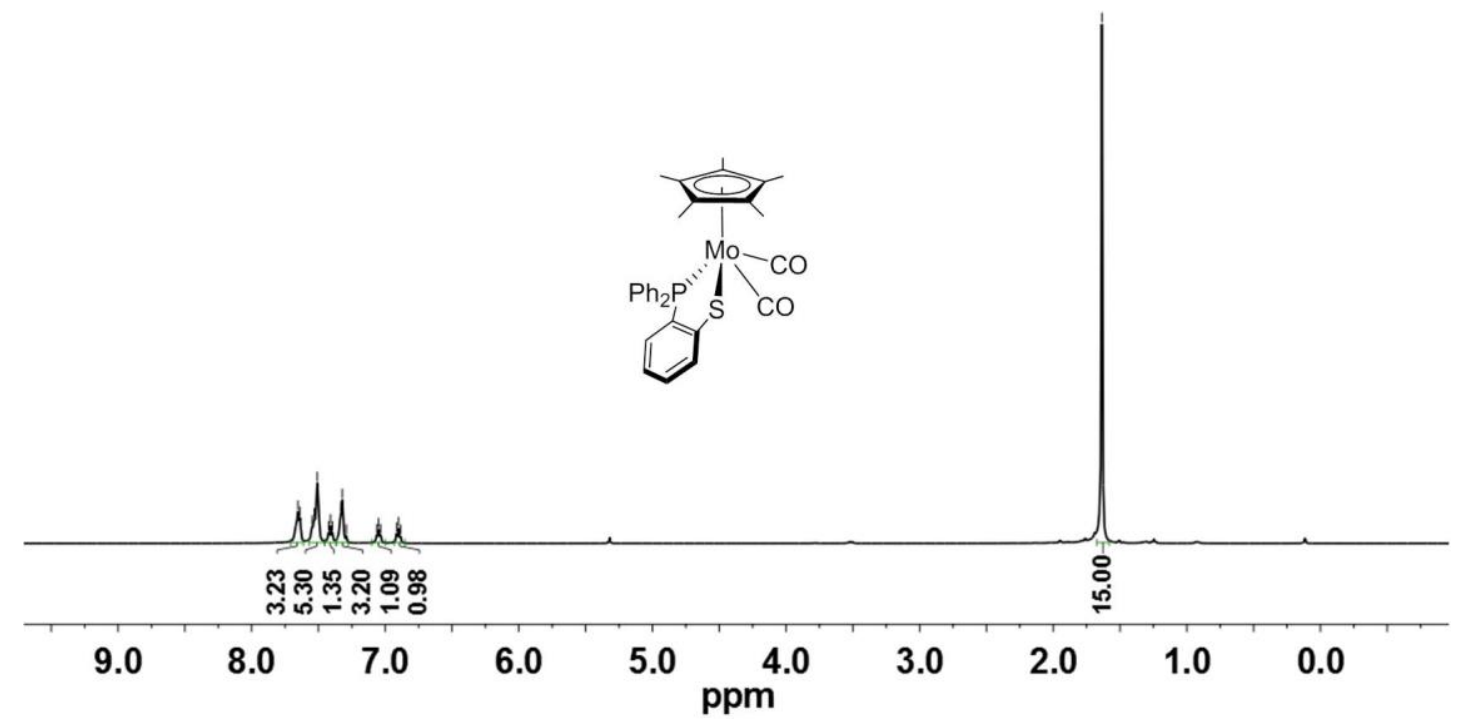

Figure S26. ${ }^{1} \mathrm{H}$ NMR spectrum of $\mathbf{1}(\mathrm{CO})_{2}$ in $\mathrm{CDCl}_{3}$. 


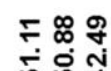

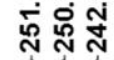

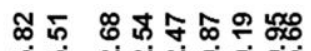

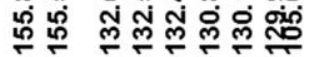

다유 m

कู่

F怘

$\stackrel{\text { ป }}{\text { ป }}$
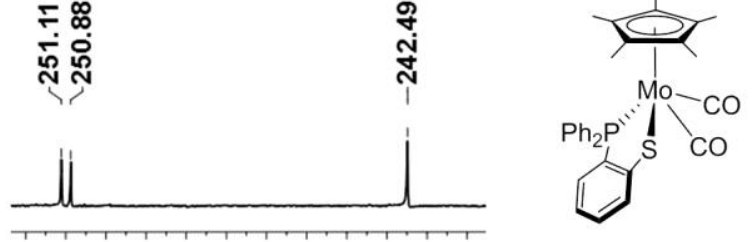

ppm

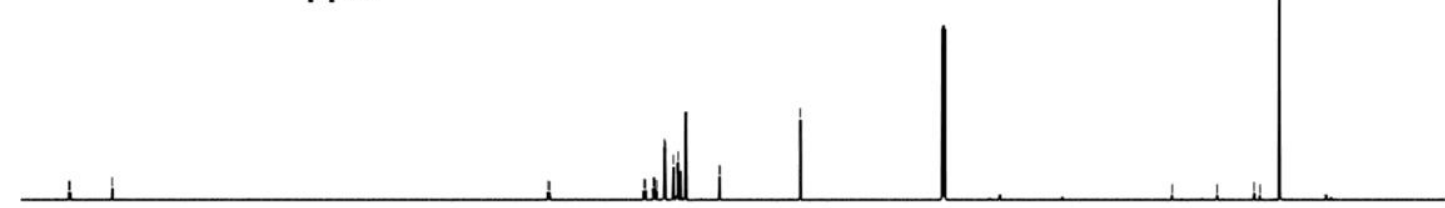

230

180

130

80

40

0

ppm

Figure S27. ${ }^{13} \mathrm{C}$ NMR spectrum of $\mathbf{1}(\mathrm{CO})_{2}$ in $\mathrm{CDCl}_{3}$. 
$m$
0
0
0

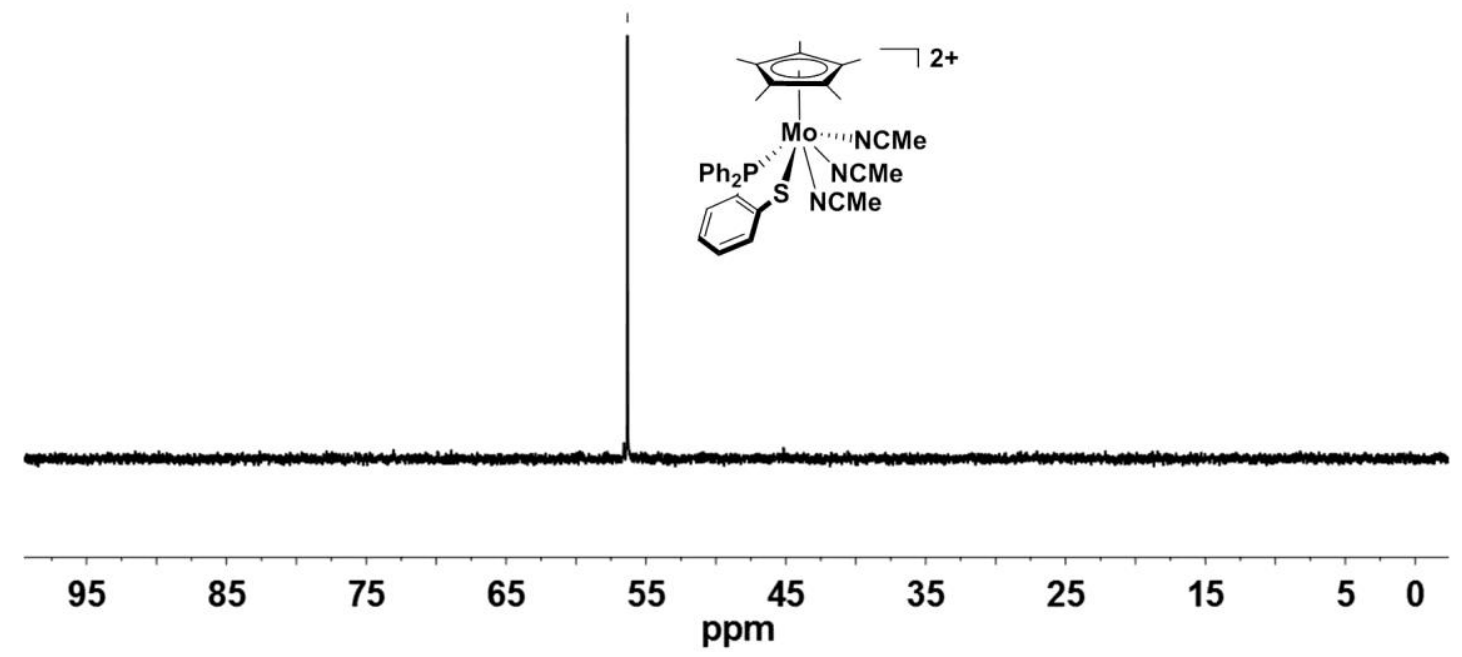

Figure S28. ${ }^{31} \mathrm{P}$ NMR spectrum of $1(\mathrm{NCMe})_{3}\left(\mathrm{BF}_{4}\right)_{2}$ in $\mathrm{CD}_{2} \mathrm{Cl}_{2}$. 


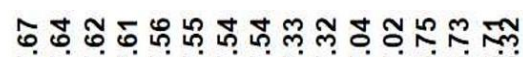

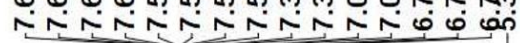

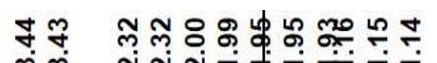

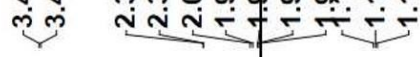

$\neg 2+$

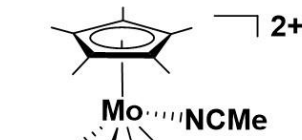

$\mathrm{Ph}_{2} \mathrm{P}^{\prime \prime}$ N $\mathrm{NCMe}$

NCMe

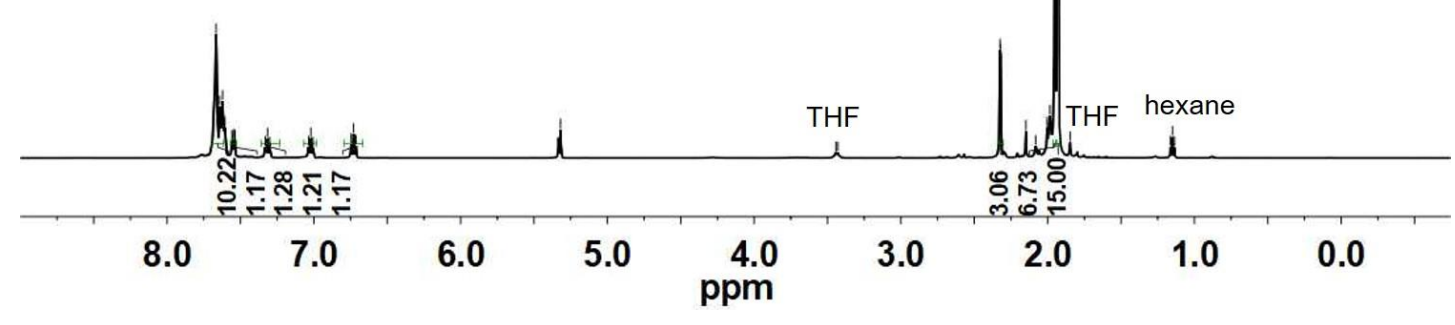

Figure S29. ${ }^{1} \mathrm{H}$ NMR spectrum of $\mathbf{1}(\mathrm{NCMe})_{3}\left(\mathrm{BF}_{4}\right)_{2}$ in $\mathrm{CD}_{2} \mathrm{Cl}_{2}$. 


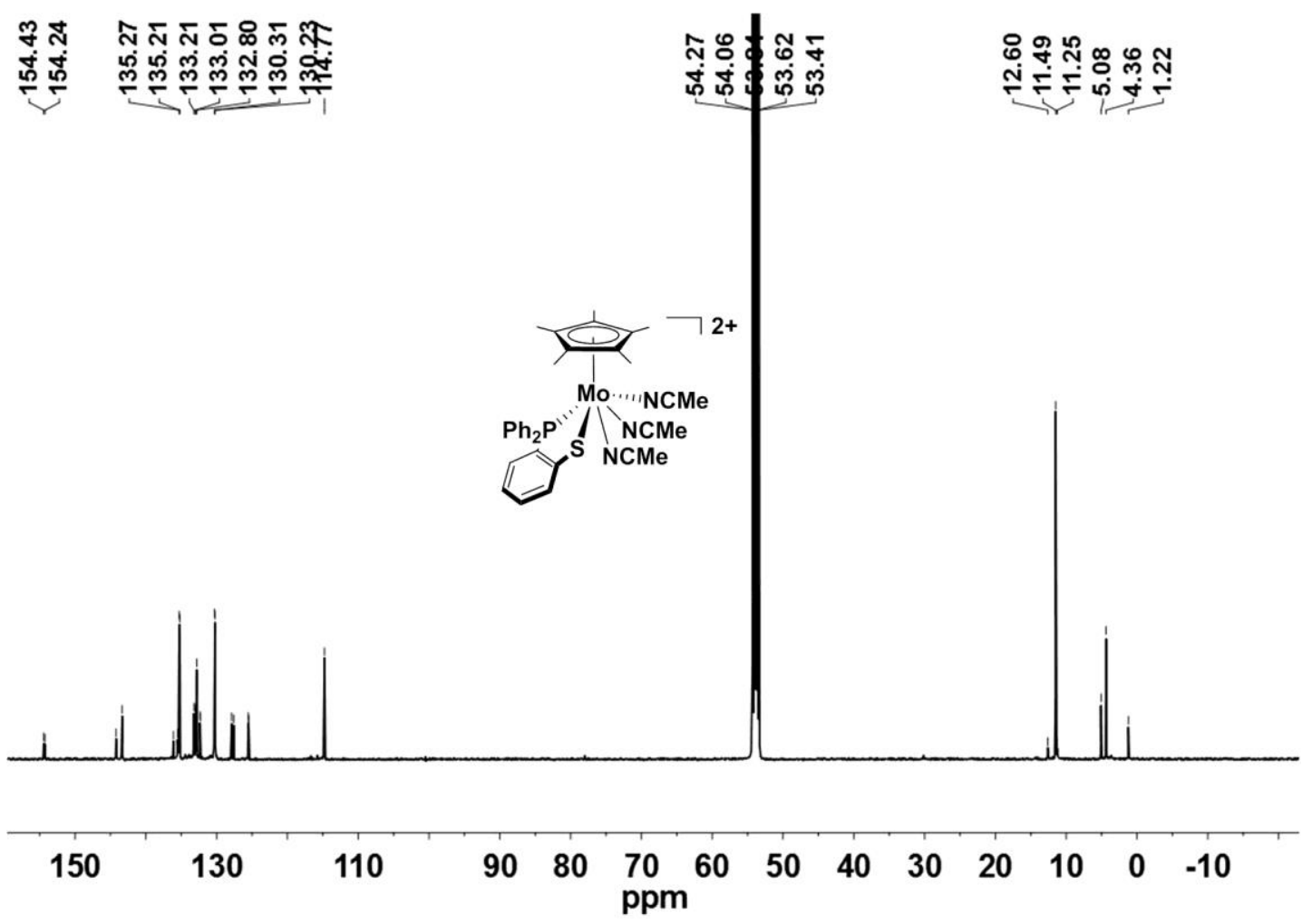

Figure S30. ${ }^{13} \mathrm{C}$ NMR spectrum of $1(\mathrm{NCMe})_{3}\left(\mathrm{BF}_{4}\right)_{2}$ in $\mathrm{CD}_{2} \mathrm{Cl}_{2}$. 
웅

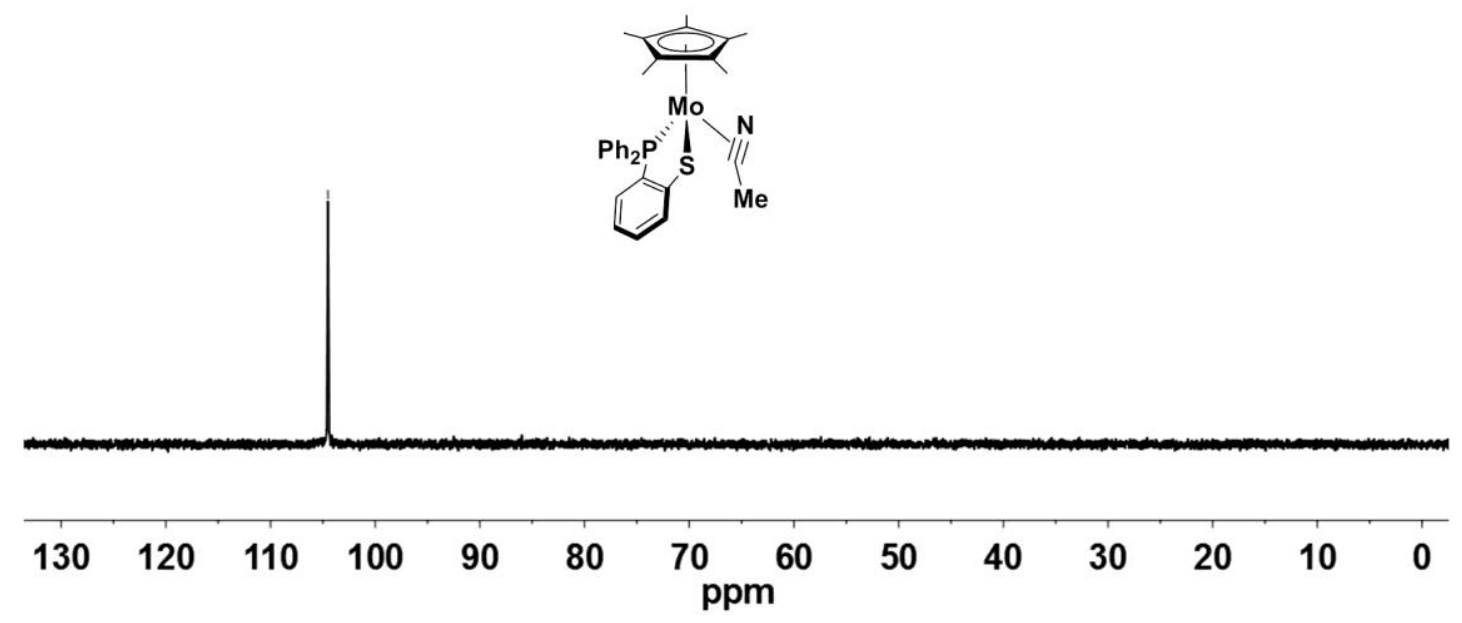

Figure S31. ${ }^{31} \mathrm{P}$ NMR spectrum of $\mathbf{1}\left(\eta^{2}-\mathrm{NCMe}\right)$ in $\mathrm{C}_{6} \mathrm{D}_{6}$. 


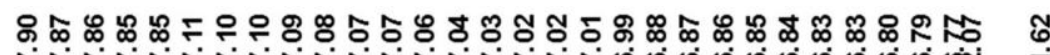

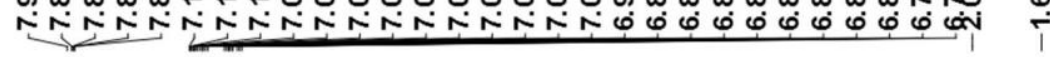

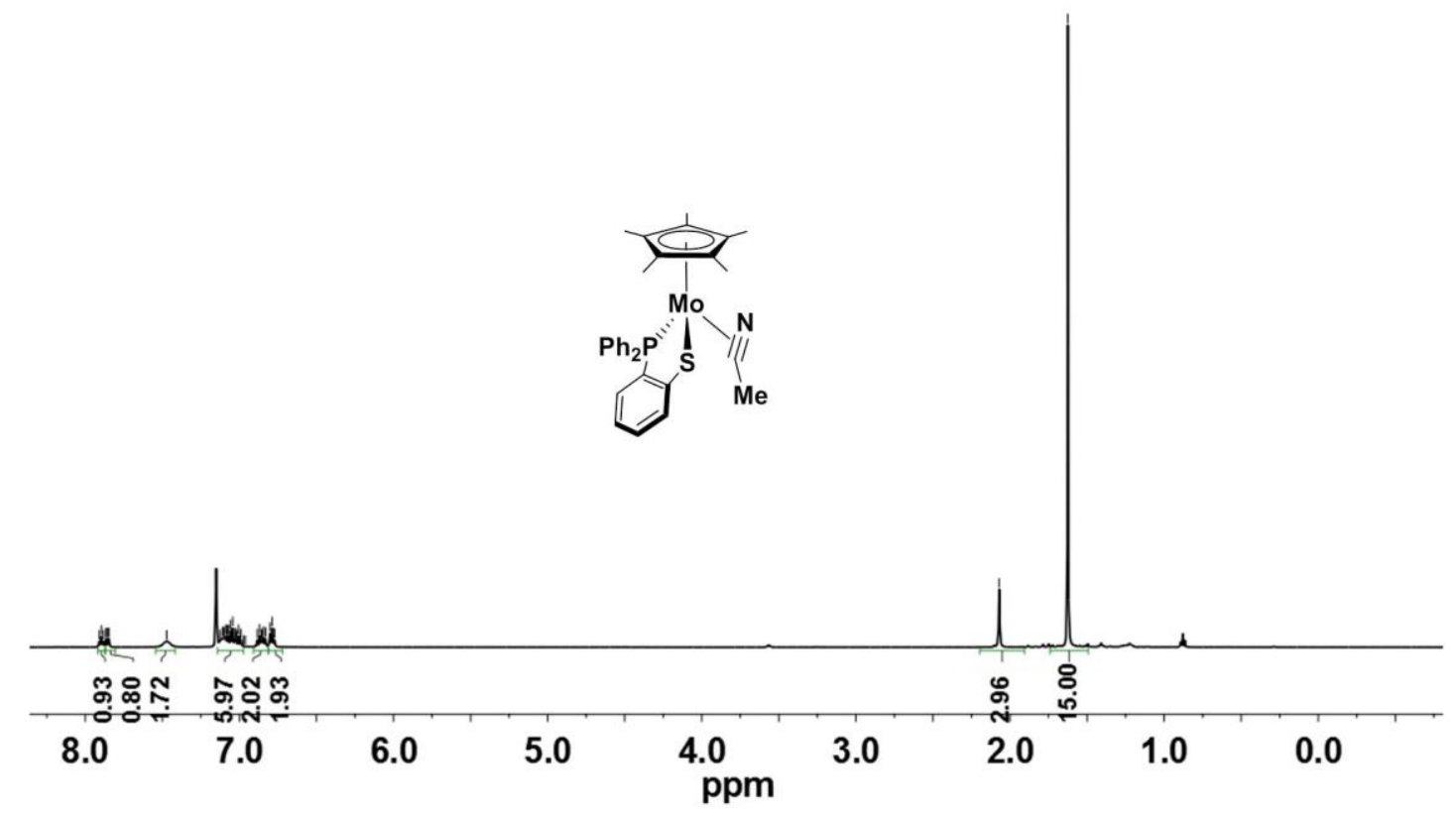

Figure S32. ${ }^{1} \mathrm{H}$ NMR spectrum of $\mathbf{1}\left(\eta^{2}-\mathrm{NCMe}\right)$ in $\mathrm{C}_{6} \mathrm{D}_{6}$. 


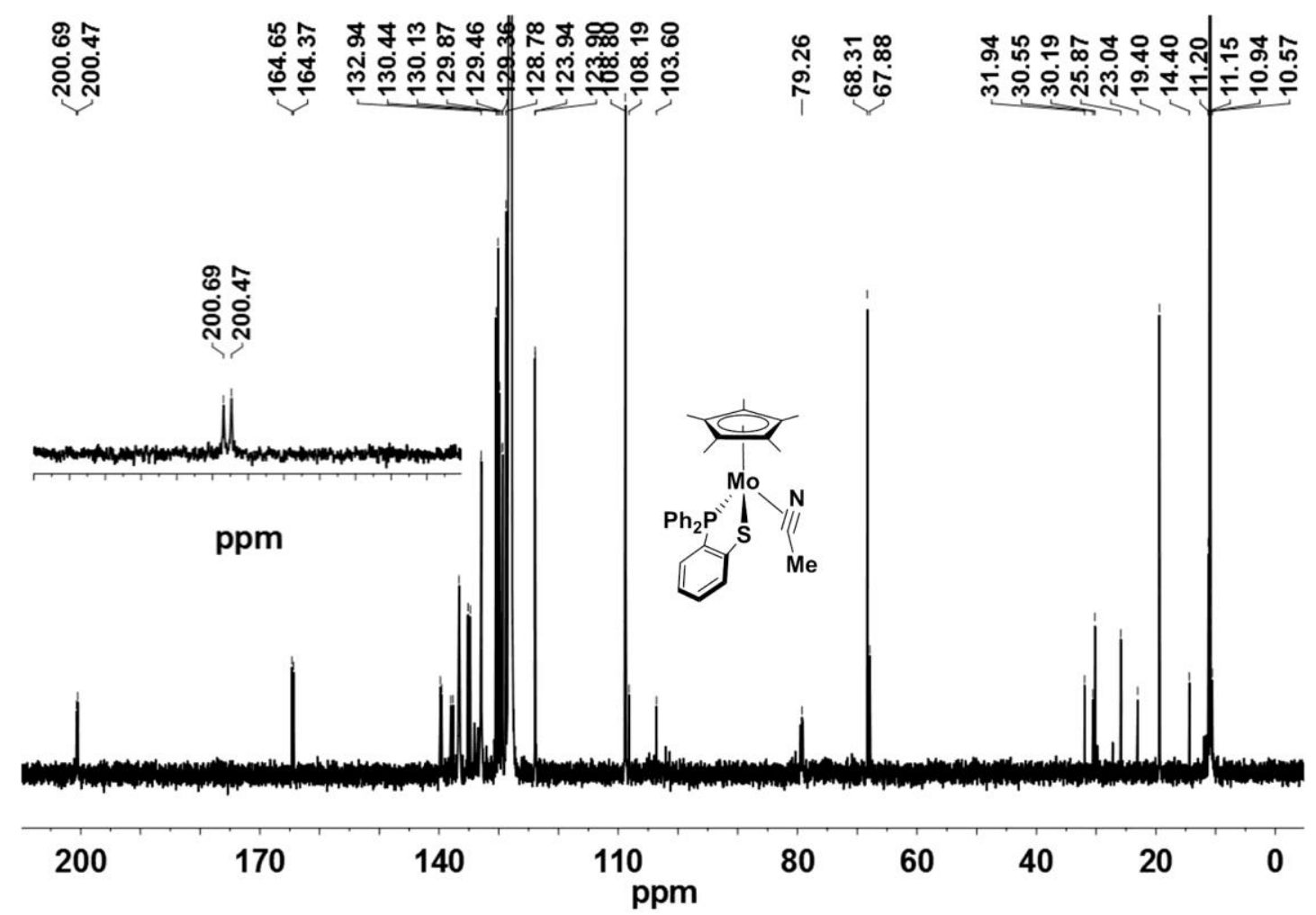

Figure S33. ${ }^{13} \mathrm{C}$ NMR spectrum of $\mathbf{1}\left(\eta^{2}-\mathrm{NCMe}\right)$ in $\mathrm{C}_{6} \mathrm{D}_{6}$. 
กั

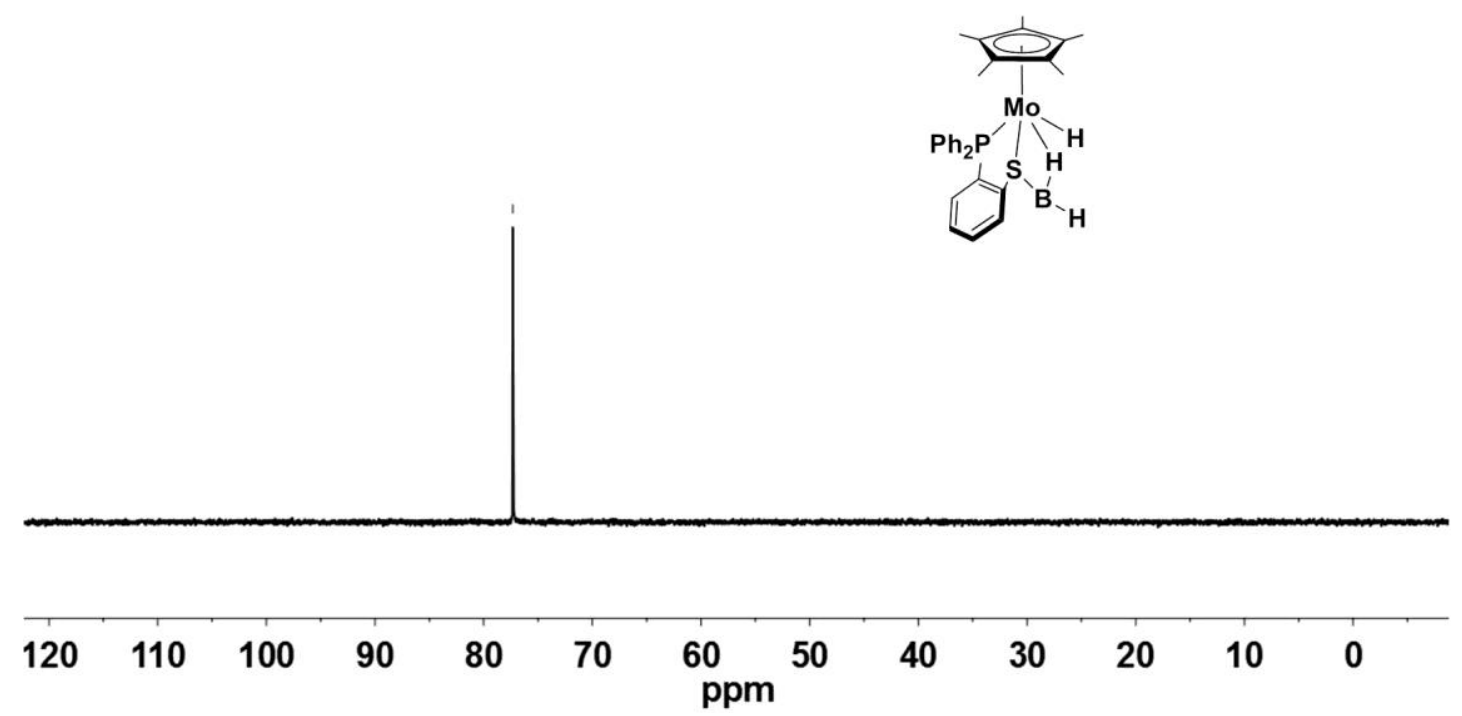

Figure S34. ${ }^{31} \mathrm{P}$ NMR spectrum of $1 \mathrm{H}\left(\mathrm{BH}_{2}\right)$ in $\mathrm{C}_{6} \mathrm{D}_{6}$. 


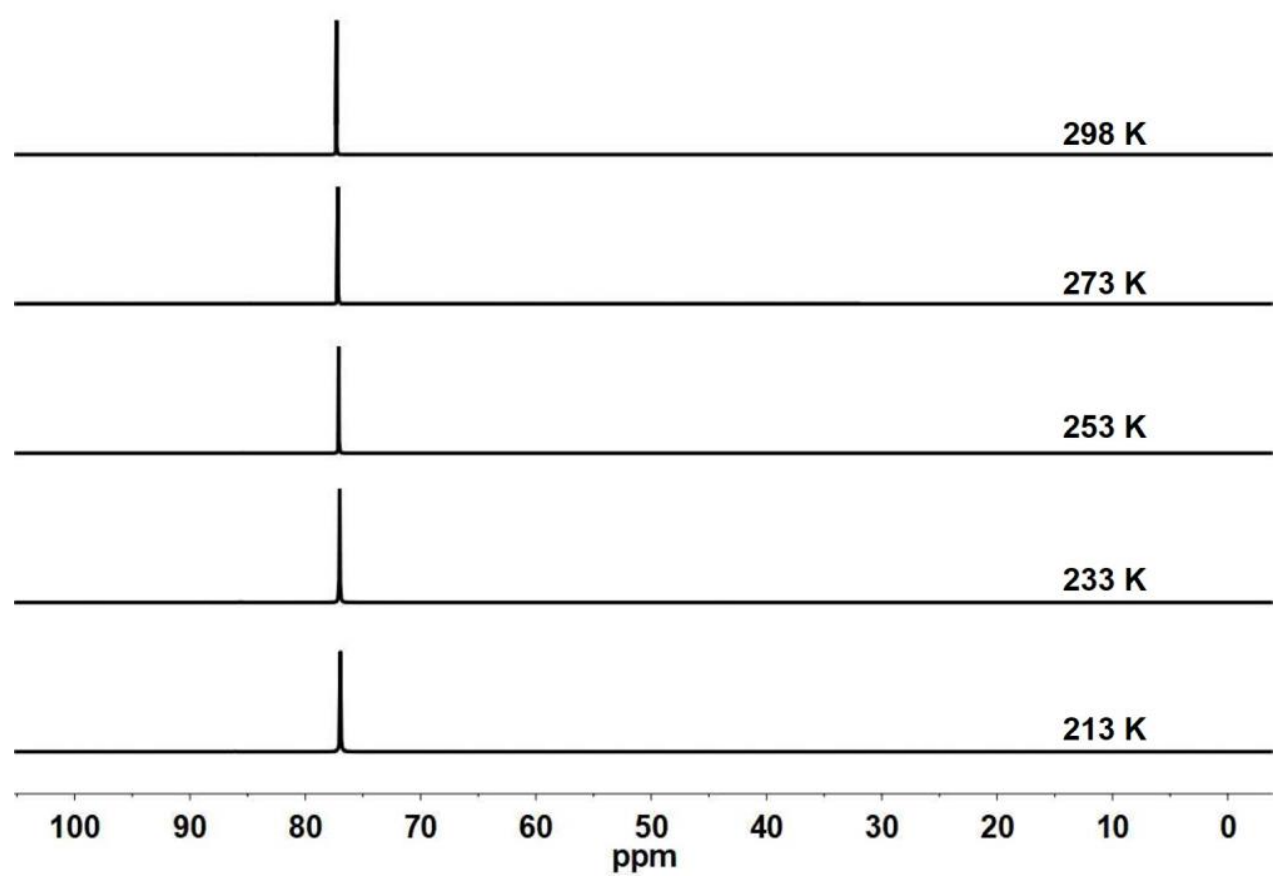

Figure S35. Variable temperature ${ }^{31} \mathrm{P}$ NMR spectra of $1 \mathrm{H}\left(\mathrm{BH}_{2}\right)$ from $298 \mathrm{~K}$ to $213 \mathrm{~K}$ in toluene- $d 8$. 


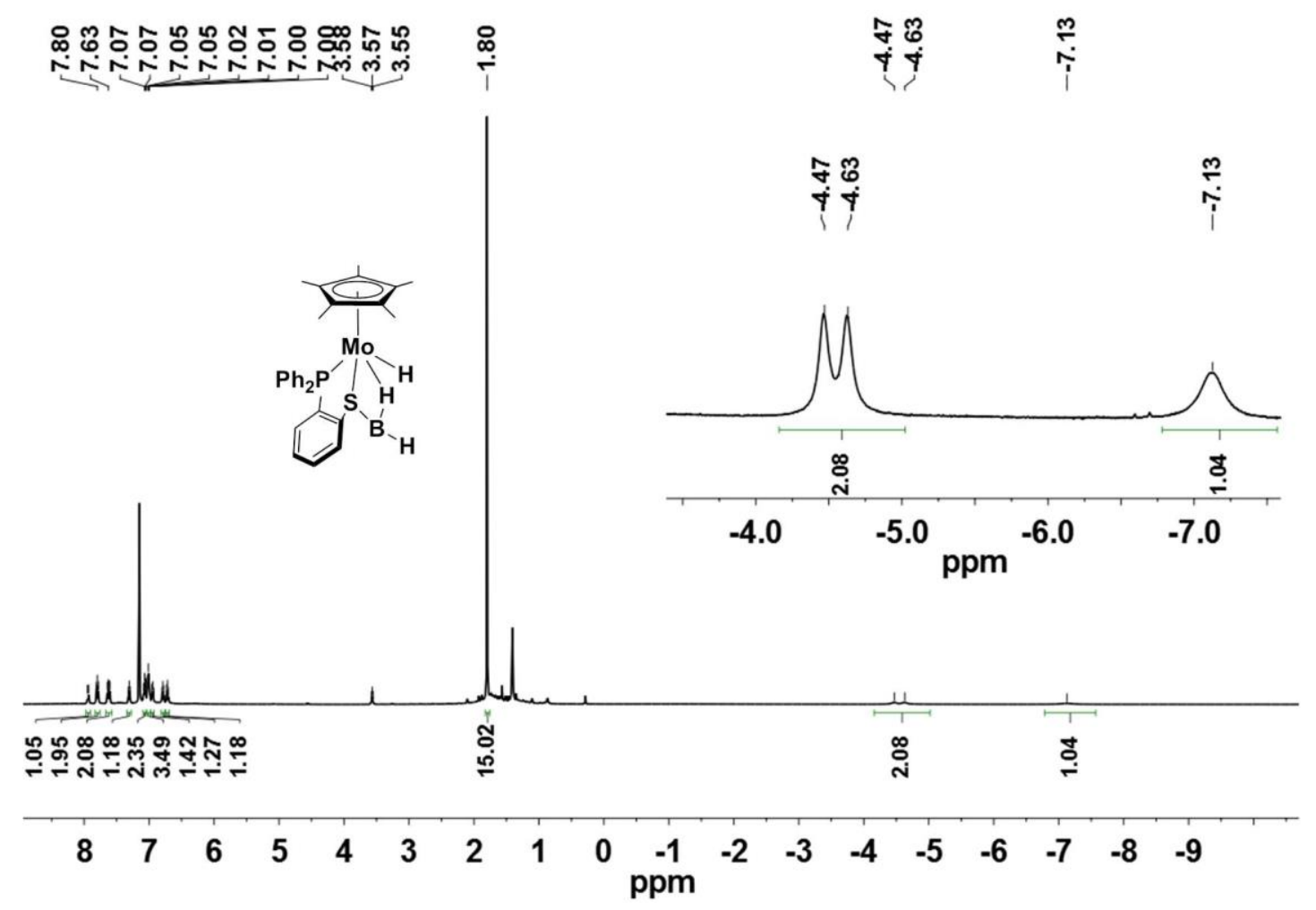

Figure S36. ${ }^{1} \mathrm{H}$ NMR spectrum of $\mathbf{1} H\left(\mathrm{BH}_{2}\right)$ in $\mathrm{C}_{6} \mathrm{D}_{6}$. 
$\underset{i}{\stackrel{1}{i}}$

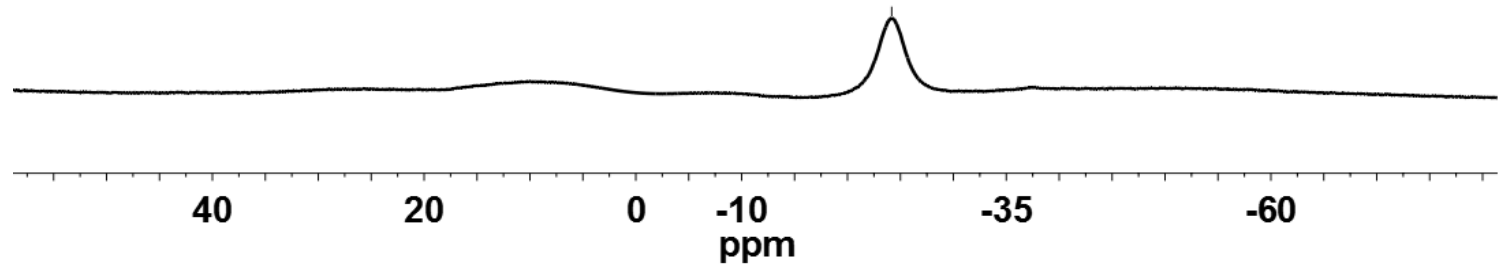

Figure S37. ${ }^{11} \mathrm{~B}$ NMR spectrum of $1 \mathrm{H}\left(\mathrm{BH}_{2}\right)$ in $\mathrm{C}_{6} \mathrm{D}_{6}$. 
ヘิ゚

$\stackrel{8}{i}$

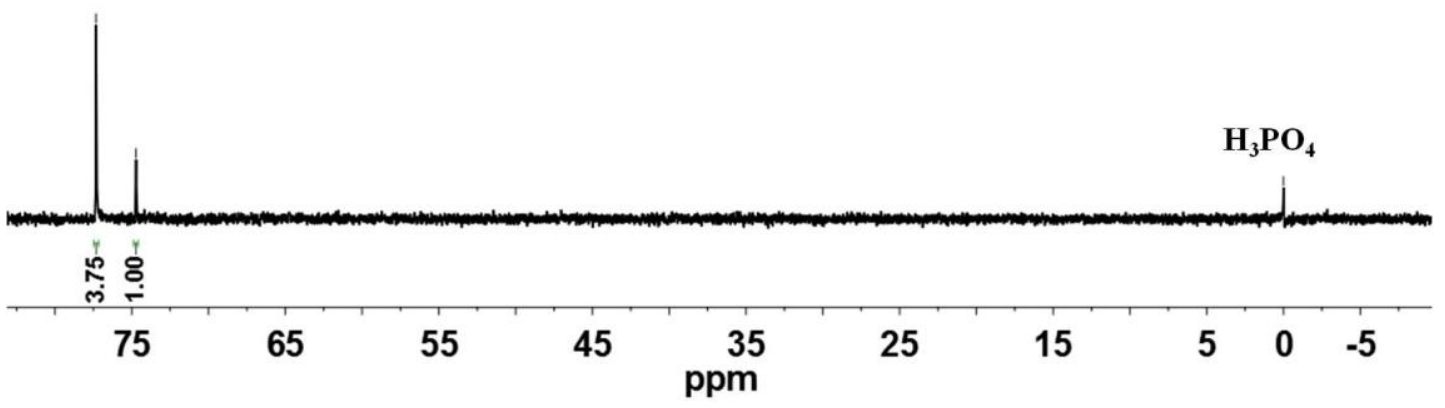

Figure S38. ${ }^{31} \mathrm{P}$ NMR spectrum for $1 \mathrm{H}\left(\mathrm{BH}_{2}\right)$ reaction with $\mathrm{NH}_{3}(\mathrm{MeOH})$ (1 equiv) at equilibrium in $\mathrm{C}_{6} \mathrm{D}_{6}$. The signal at $\delta 0.0$ is the internal standard of $85 \% \mathrm{H}_{3} \mathrm{PO}_{4}$ in capillary. 


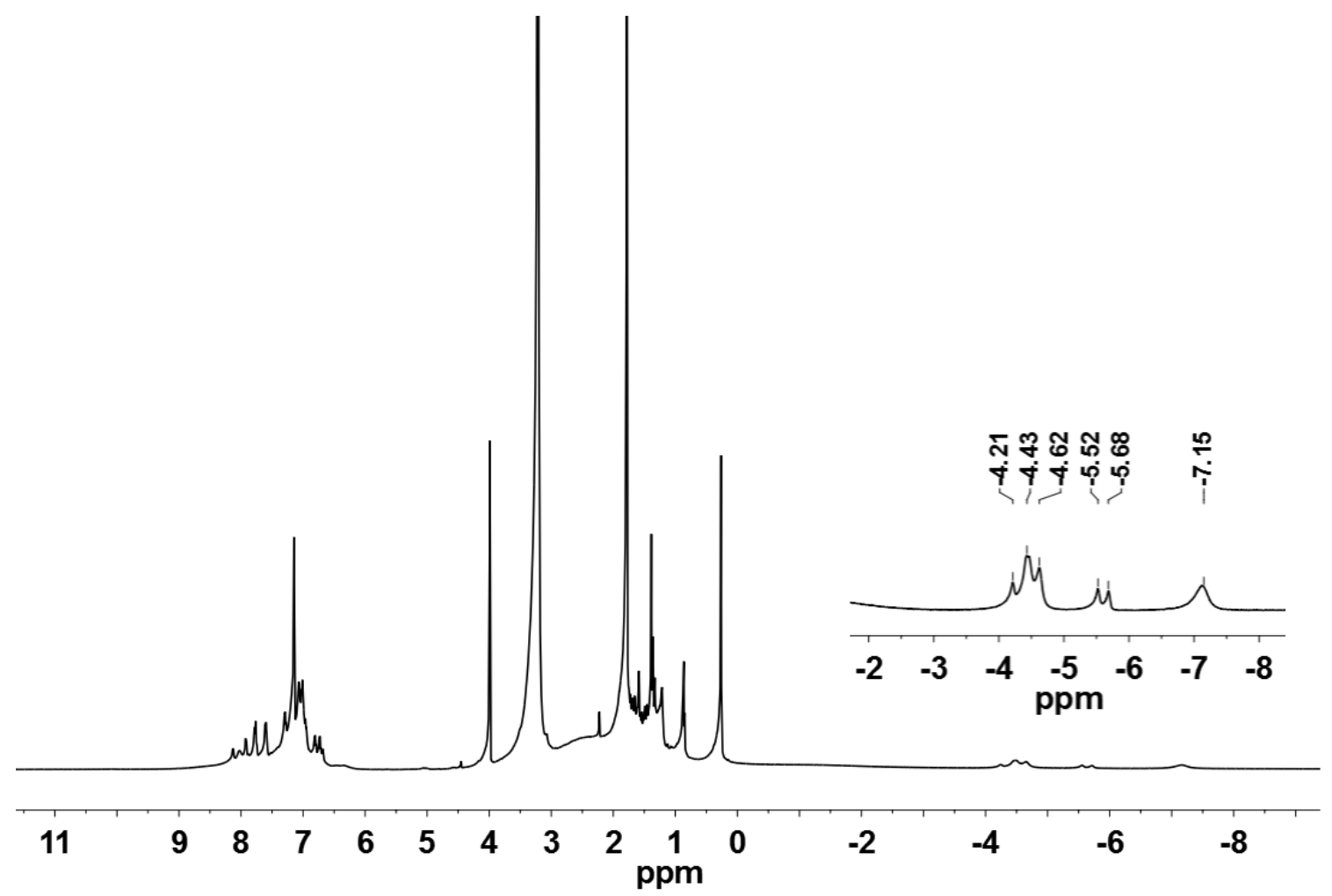

Figure S39. ${ }^{1} \mathrm{H}$ NMR spectrum for $1 \mathrm{H}\left(\mathrm{BH}_{2}\right)$ (1 equiv) reaction with $\mathrm{NH}_{3}(\mathrm{MeOH})(1$ equiv) at equilibrium in $\mathrm{C}_{6} \mathrm{D}_{6}$. 


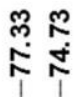

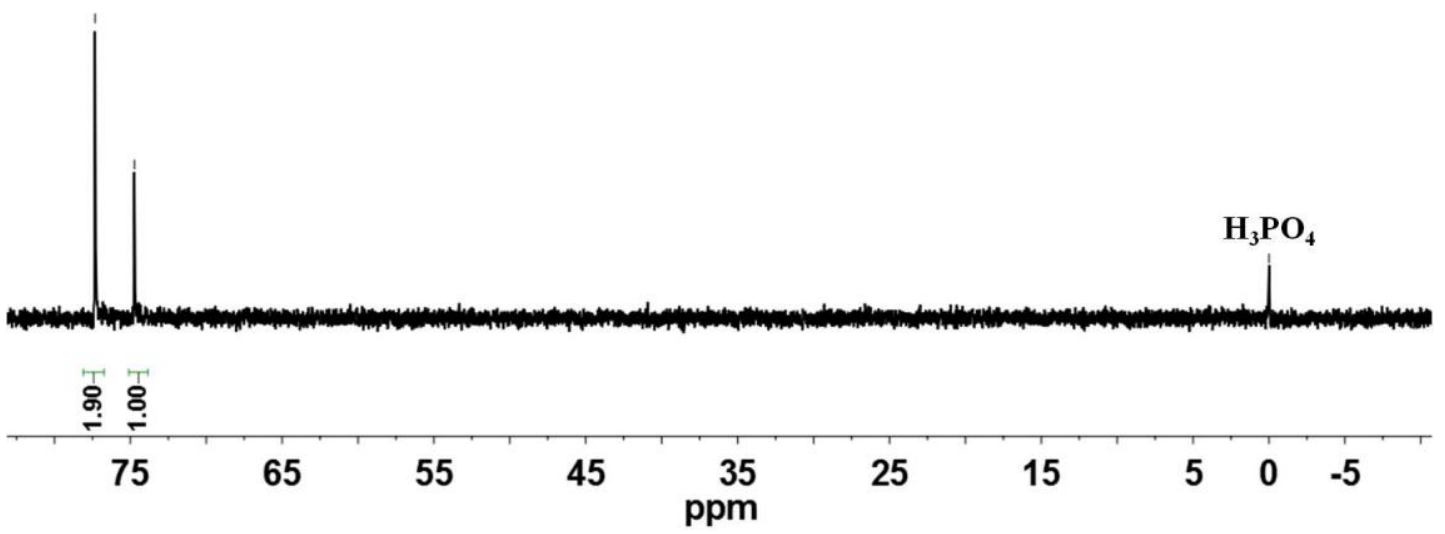

Figure S40. ${ }^{1} \mathrm{H}$ NMR spectrum for $1 \mathrm{H}\left(\mathrm{BH}_{2}\right)$ reaction with $\mathrm{NH}_{3}(\mathrm{MeOH})$ (2 equiv) at equilibrium in $\mathrm{C}_{6} \mathrm{D}_{6}$. The signal at $\delta 0.0$ is the internal standard of $85 \% \mathrm{H}_{3} \mathrm{PO}_{4}$ in capillary. 


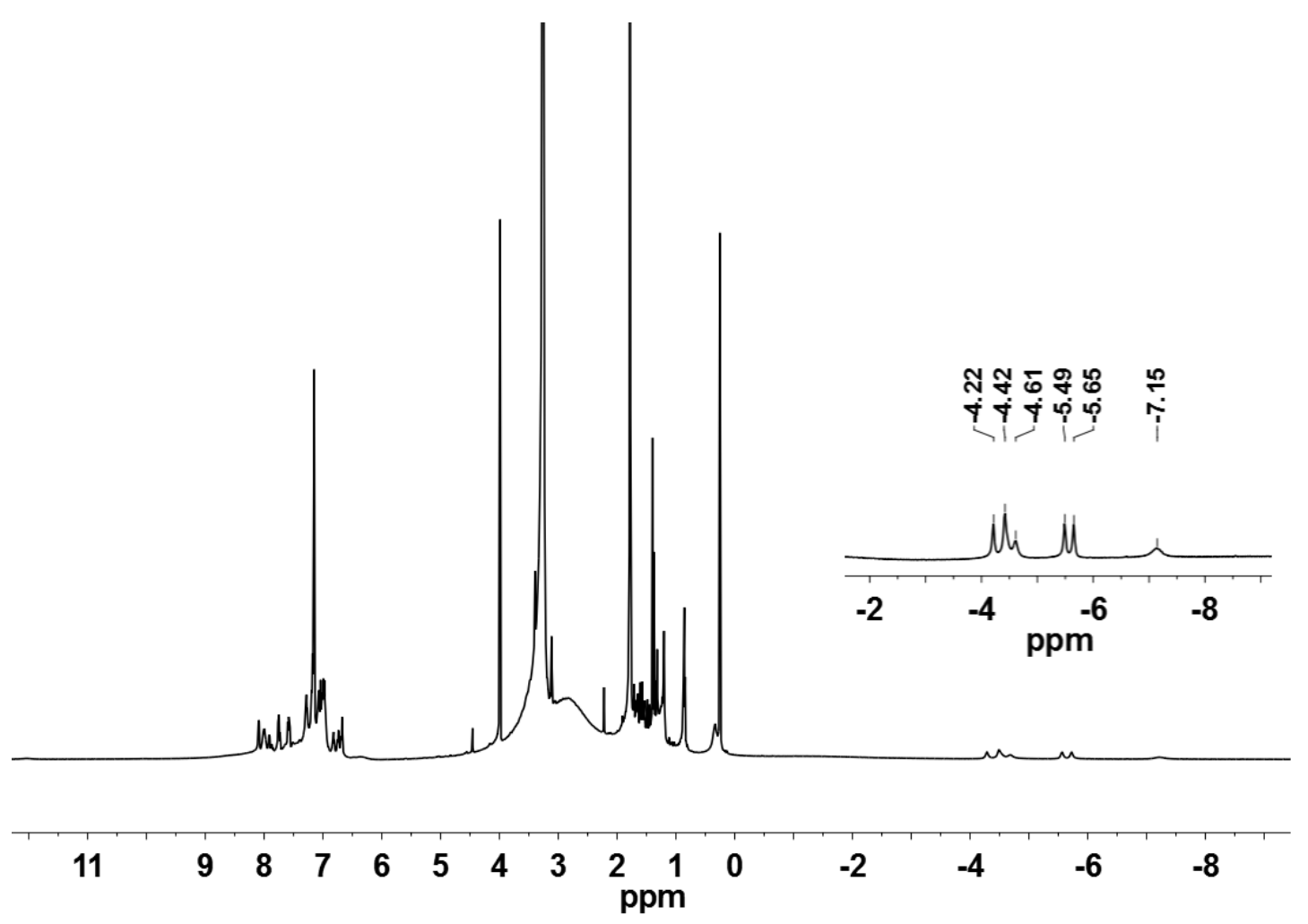

Figure S41. ${ }^{1} \mathrm{H}$ NMR spectrum for $1 \mathrm{H}\left(\mathrm{BH}_{2}\right)$ reaction with $\mathrm{NH}_{3}(\mathrm{MeOH})$ (2 equiv) at equilibrium in $\mathrm{C}_{6} \mathrm{D}_{6}$. 


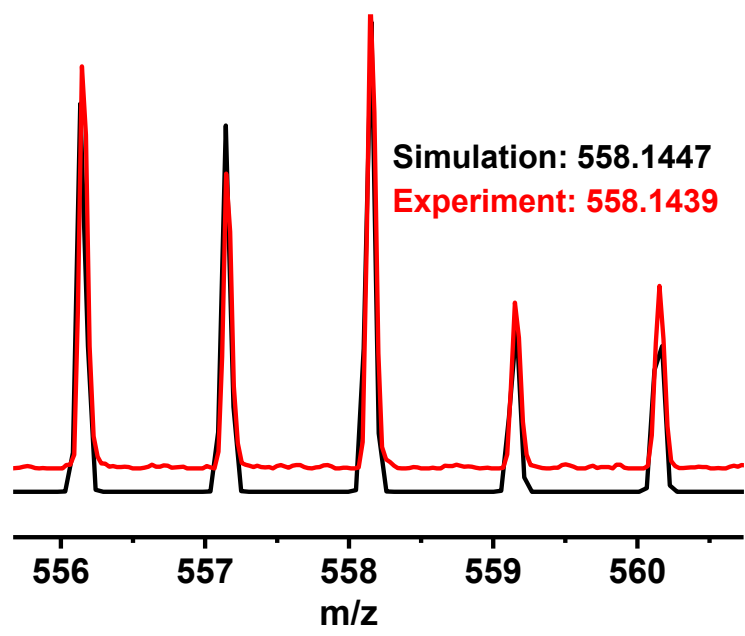

Figure S42. ESI-MS mass spectrum $1 \mathrm{H}\left(\mathrm{H}_{2} \mathrm{~B} \cdot \mathrm{NH}_{3}\right)$.

Result: Calcd for $\left[1 \mathrm{H}\left(\mathrm{H}_{2} \mathrm{~B} \cdot \mathrm{NH}_{3}\right)+\mathrm{H}\right]^{+}, 558.1447$; found, 558.1439 . 
9. NMR Spectra of the hydrogenation products
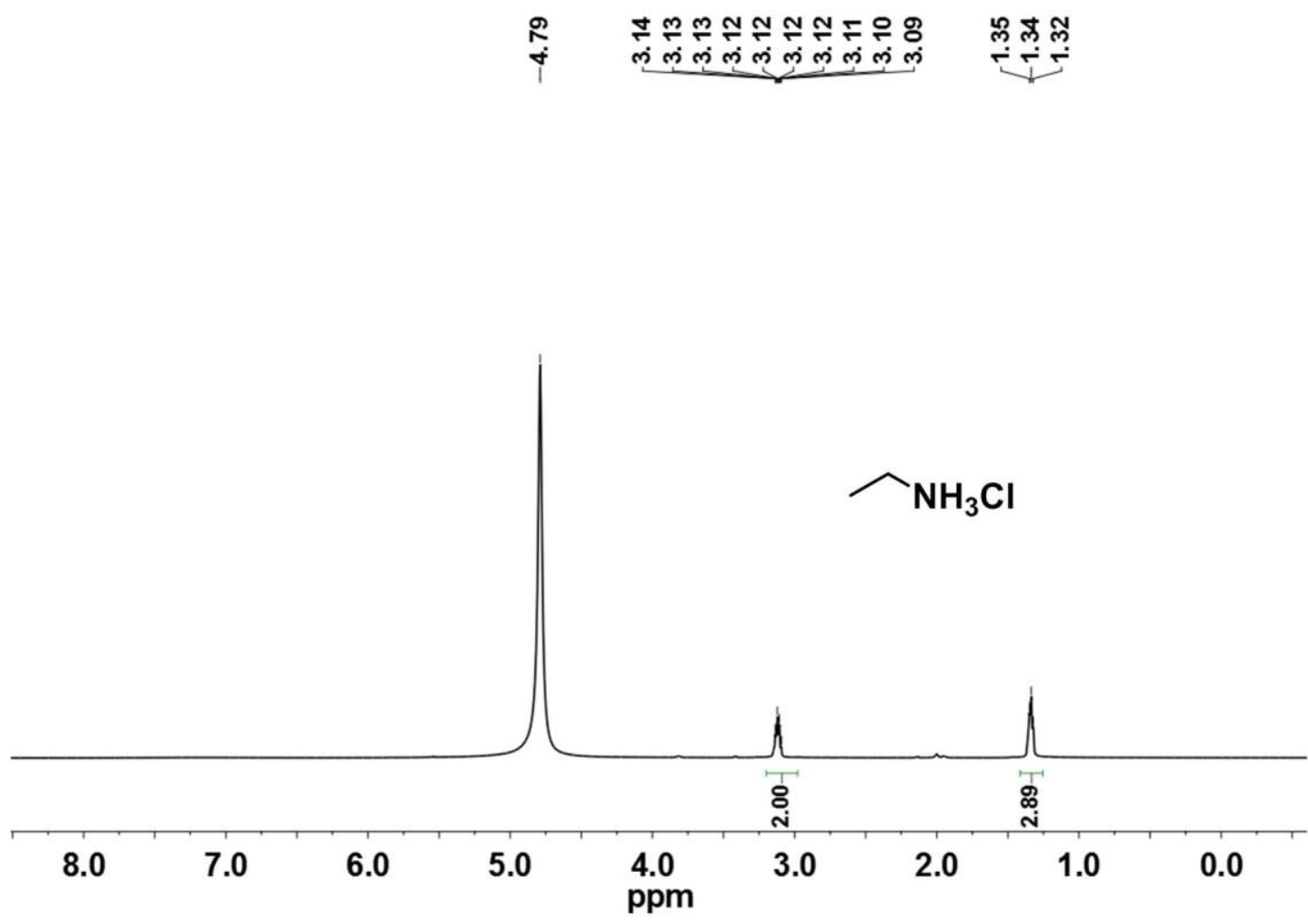

Figure S43. ${ }^{1} \mathrm{H}$ NMR spectrum of ethylamine hydrochloride (4a) in $\mathrm{D}_{2} \mathrm{O}$.
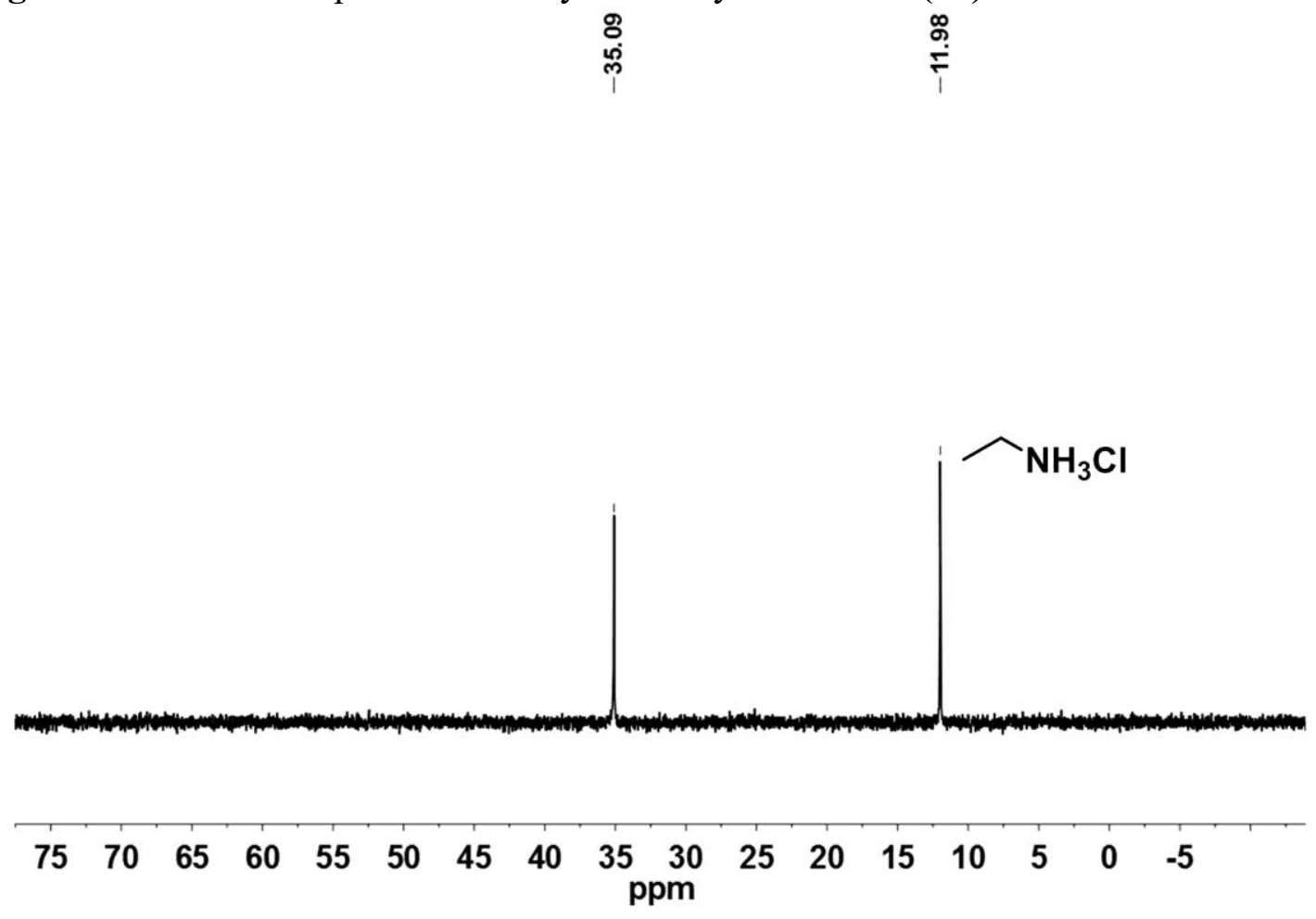

Figure S44. ${ }^{13} \mathrm{C}$ NMR spectrum of ethylamine hydrochloride (4a) in $\mathrm{D}_{2} \mathrm{O}$. 


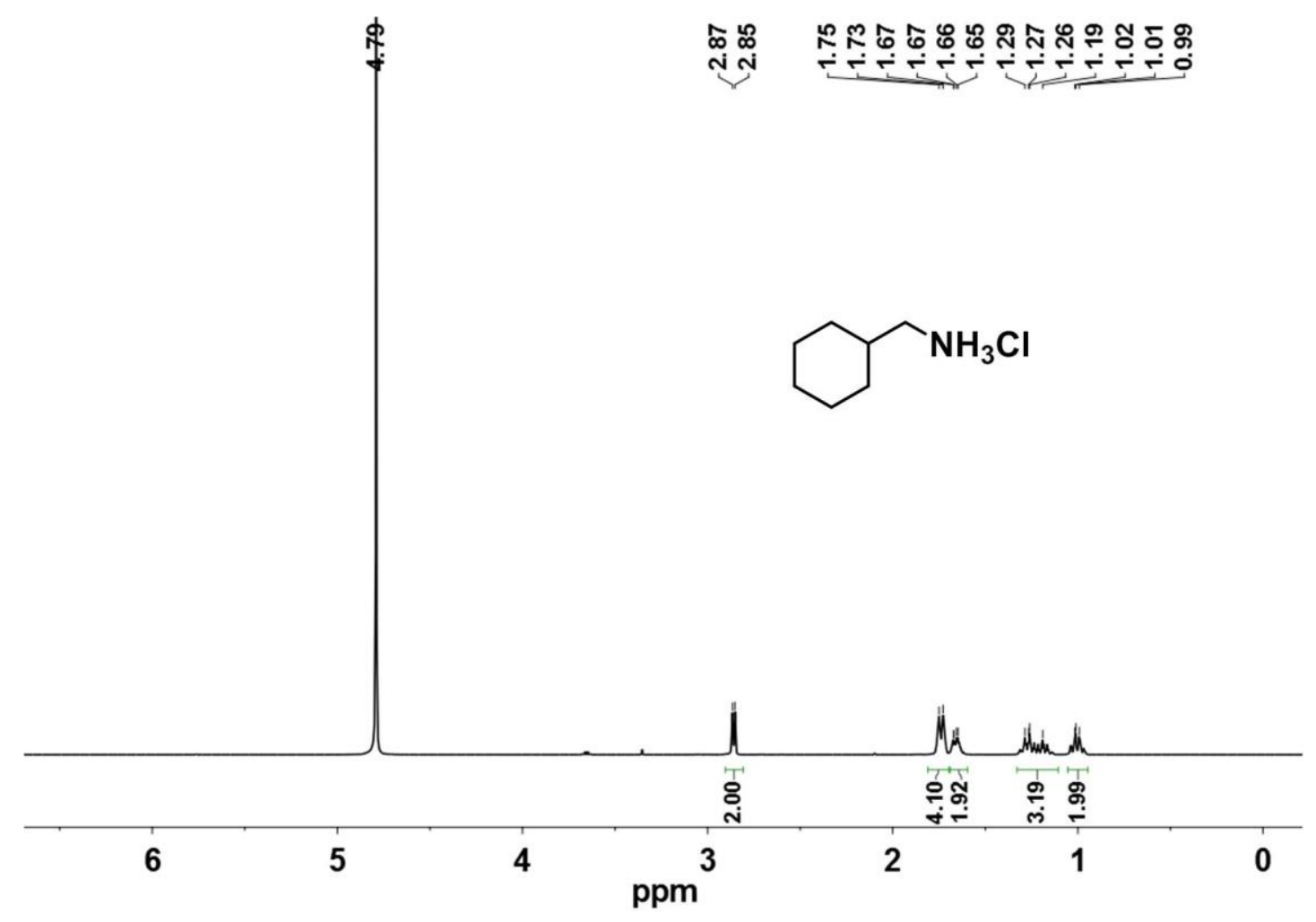

Figure S45. ${ }^{1} \mathrm{H}$ NMR spectrum of cyclohexylmethylamine hydrochloride (4b) in $\mathrm{D}_{2} \mathrm{O}$.

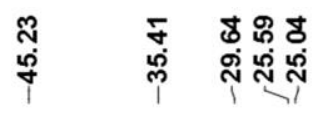

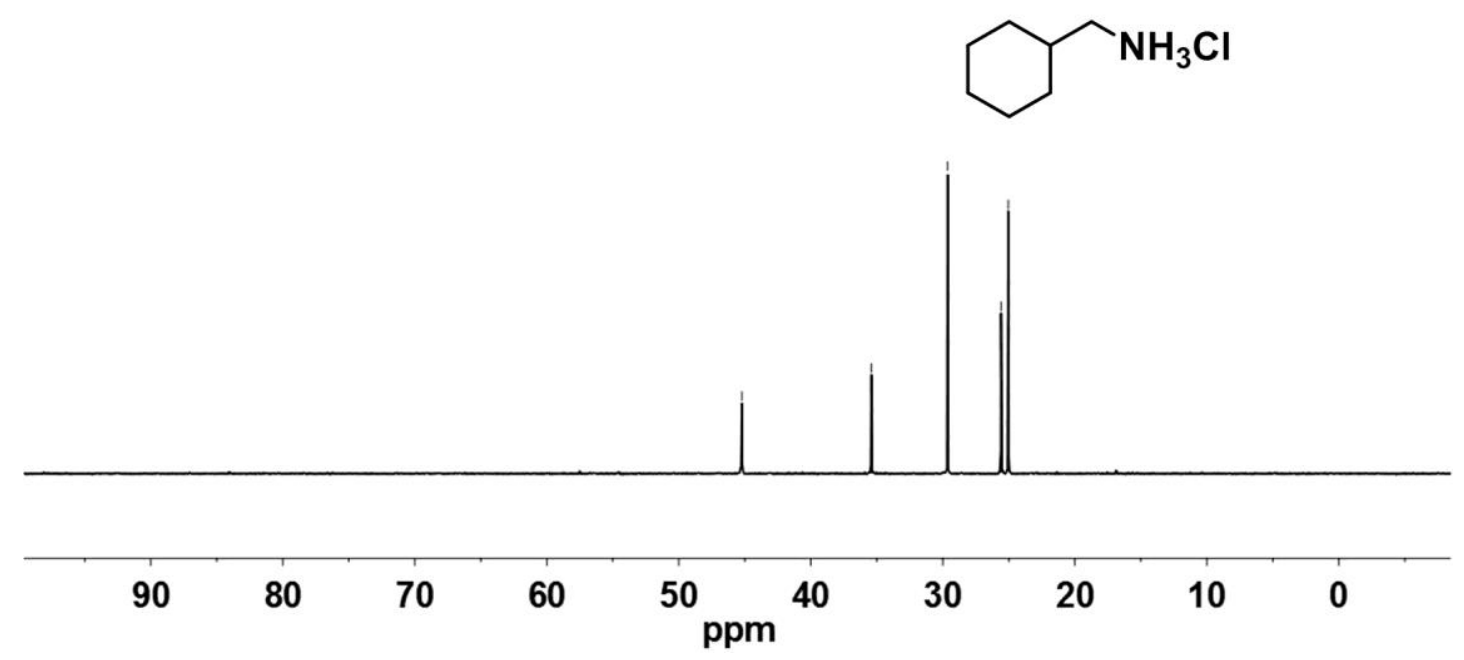

Figure S46. ${ }^{13} \mathrm{C}$ NMR spectrum of cyclohexylmethylamine hydrochloride (4b) in $\mathrm{D}_{2} \mathrm{O}$. 


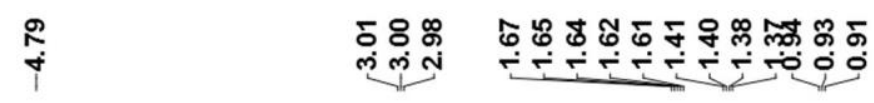

$\sim \mathrm{NH}_{3} \mathrm{Cl}$

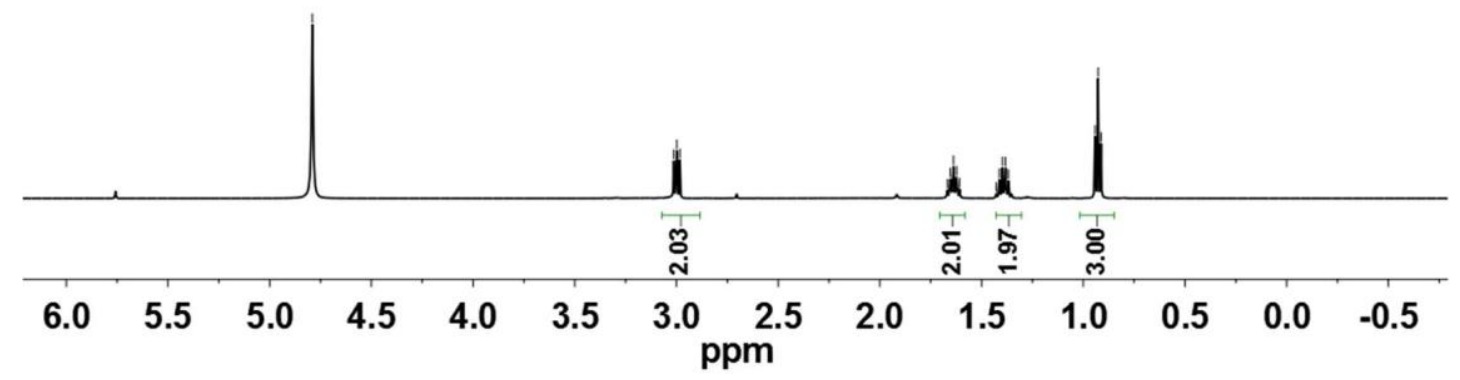

Figure S47. ${ }^{1} \mathrm{H}$ NMR spectrum of $n$-butylamine hydrochloride (4c) in $\mathrm{D}_{2} \mathrm{O}$.

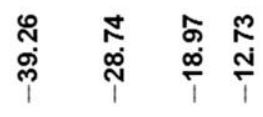

$\widetilde{\mathrm{NH}_{3} \mathrm{Cl}}$

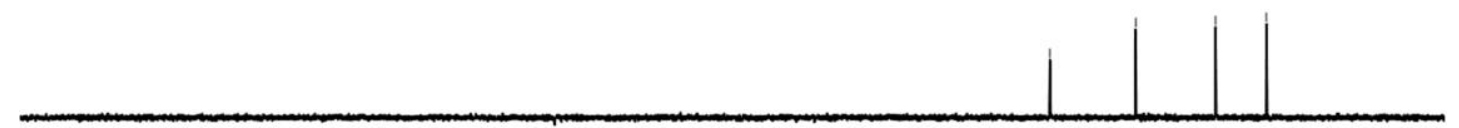

$\begin{array}{lllllllllllll}150 & 130 & 110 & 90 & \begin{array}{l}80 \\ \mathrm{ppm}\end{array} & 70 & 60 & 50 & 40 & 30 & 20 & 10 & 0\end{array}$

Figure S48. ${ }^{13} \mathrm{C}$ NMR spectrum of $n$-butylamine hydrochloride (4c) in $\mathrm{D}_{2} \mathrm{O}$. 


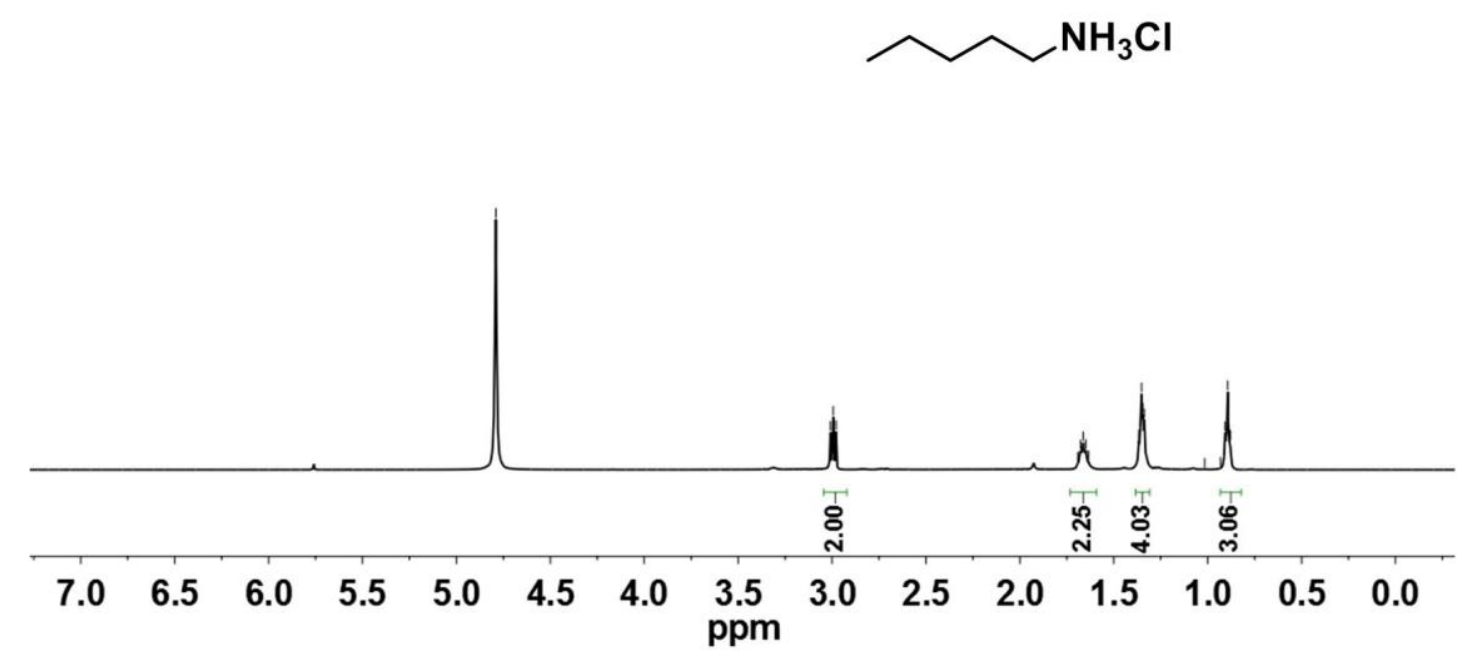

Figure S49. ${ }^{1} \mathrm{H}$ NMR spectrum of pentamine hydrochloride (4d) in $\mathrm{D}_{2} \mathrm{O}$.

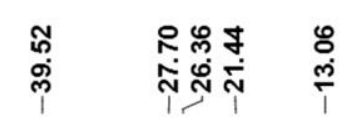

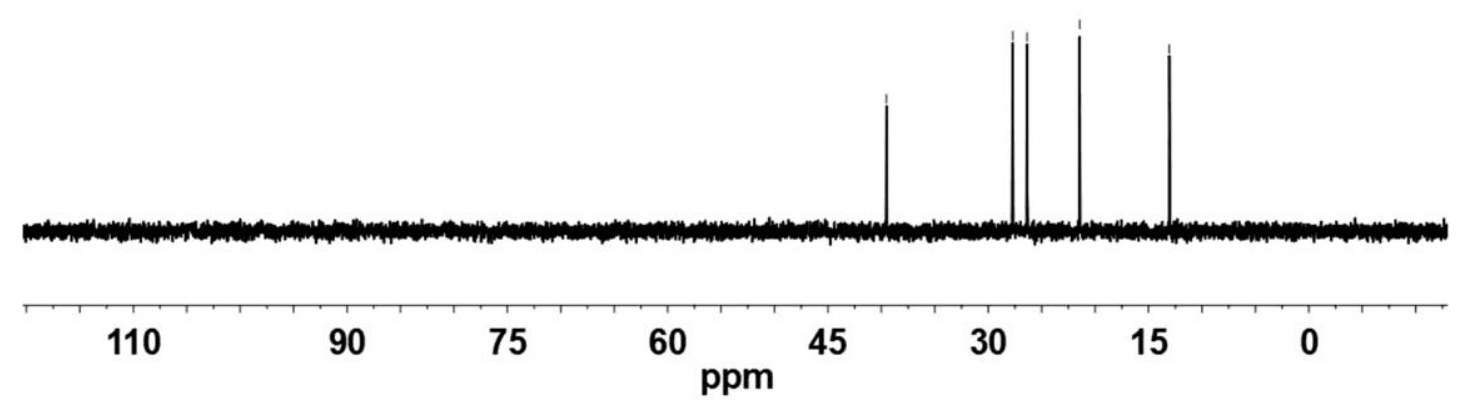

Figure S50. ${ }^{13} \mathrm{C}$ NMR spectrum of pentamine hydrochloride (4d) in $\mathrm{D}_{2} \mathrm{O}$. 


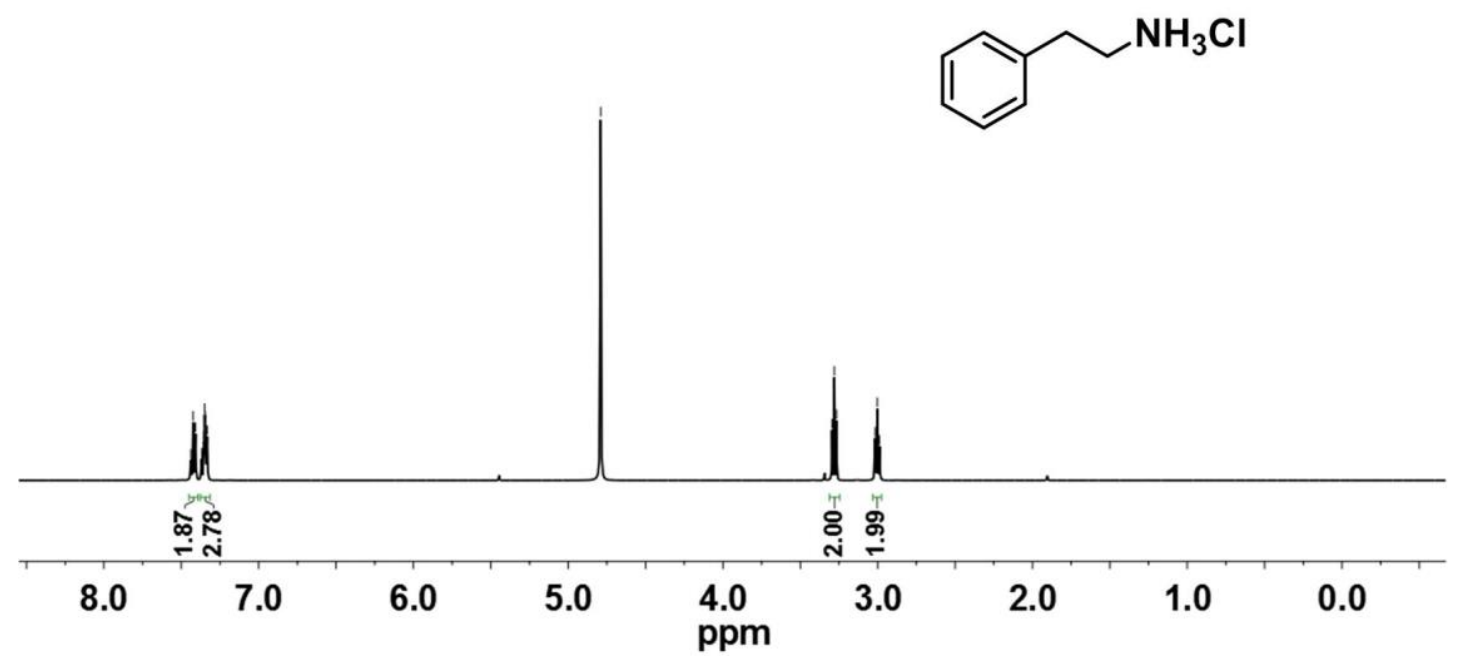

Figure S51. ${ }^{1} \mathrm{H}$ NMR spectrum of $\beta$-phenylethylamine hydrochloride (4e) in $\mathrm{D}_{2} \mathrm{O}$.

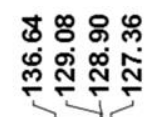

๕.

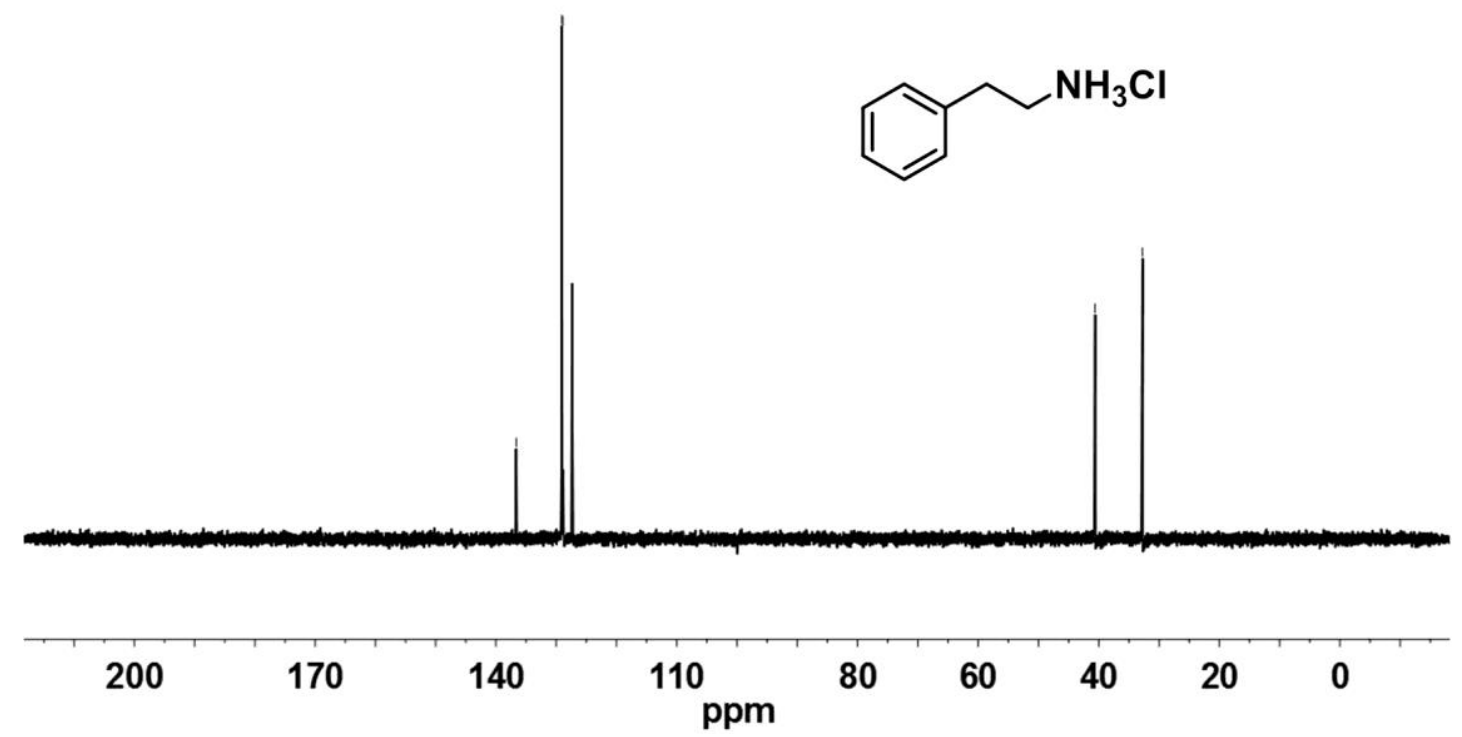

Figure S52. ${ }^{13} \mathrm{C}$ NMR spectrum of $\beta$-phenylethylamine hydrochloride (4e) in $\mathrm{D}_{2} \mathrm{O}$. 

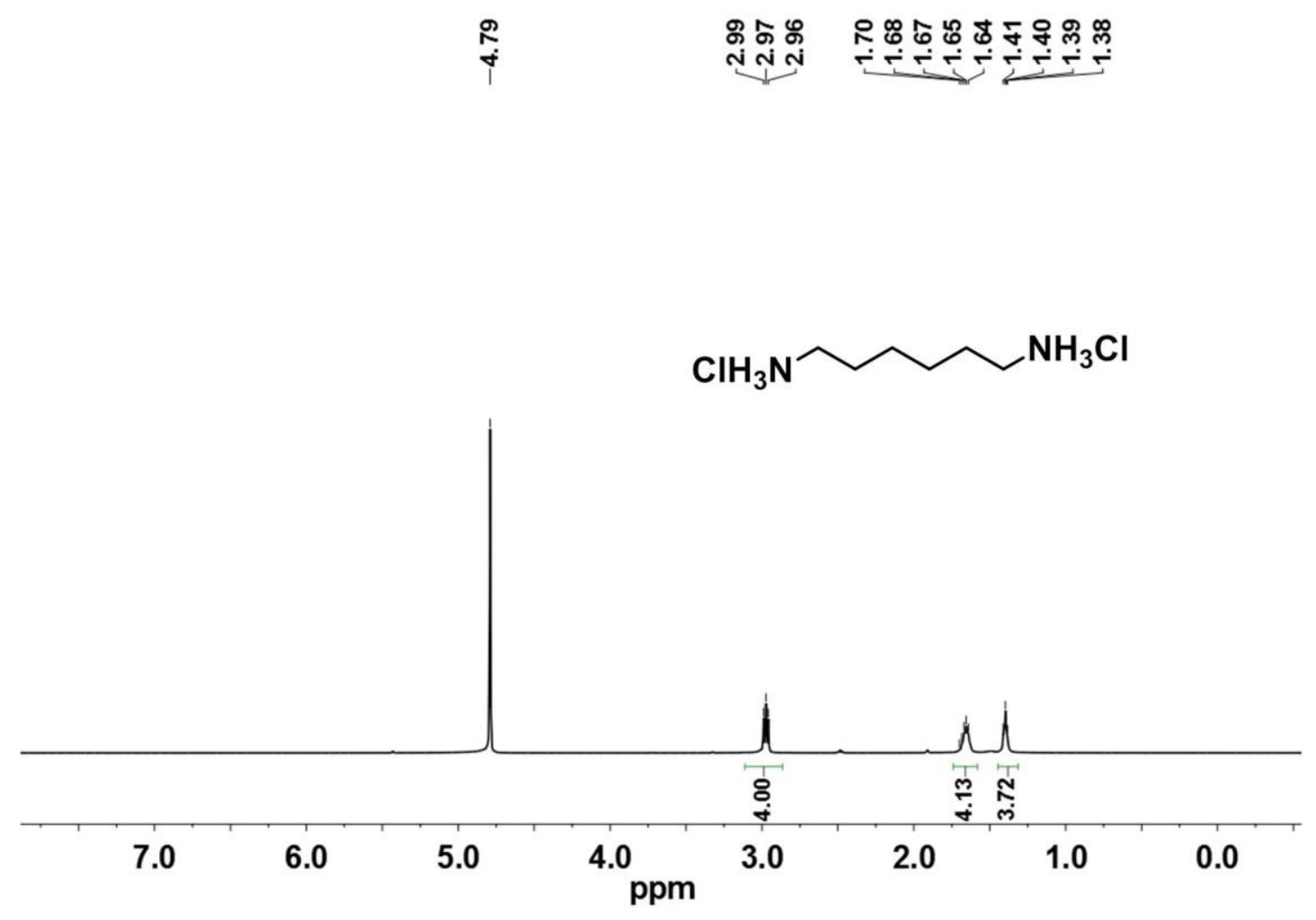

Figure S53. ${ }^{1} \mathrm{H}$ NMR spectrum of hexamethylenediamine dihydrochloride (4f) in $\mathrm{D}_{2} \mathrm{O}$.

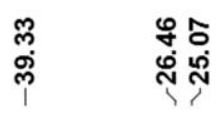
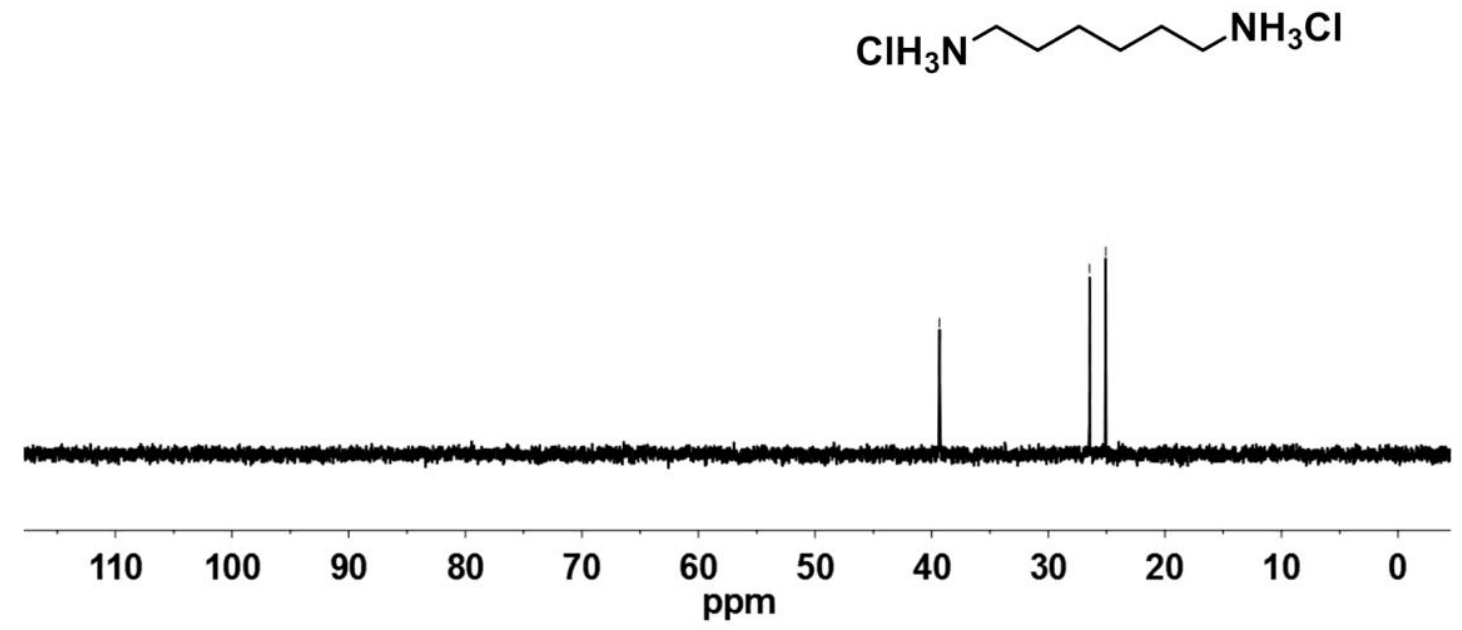

Figure S54. ${ }^{13} \mathrm{C}$ NMR spectrum of hexamethylenediamine dihydrochloride (4f) in $\mathrm{D}_{2} \mathrm{O}$. 

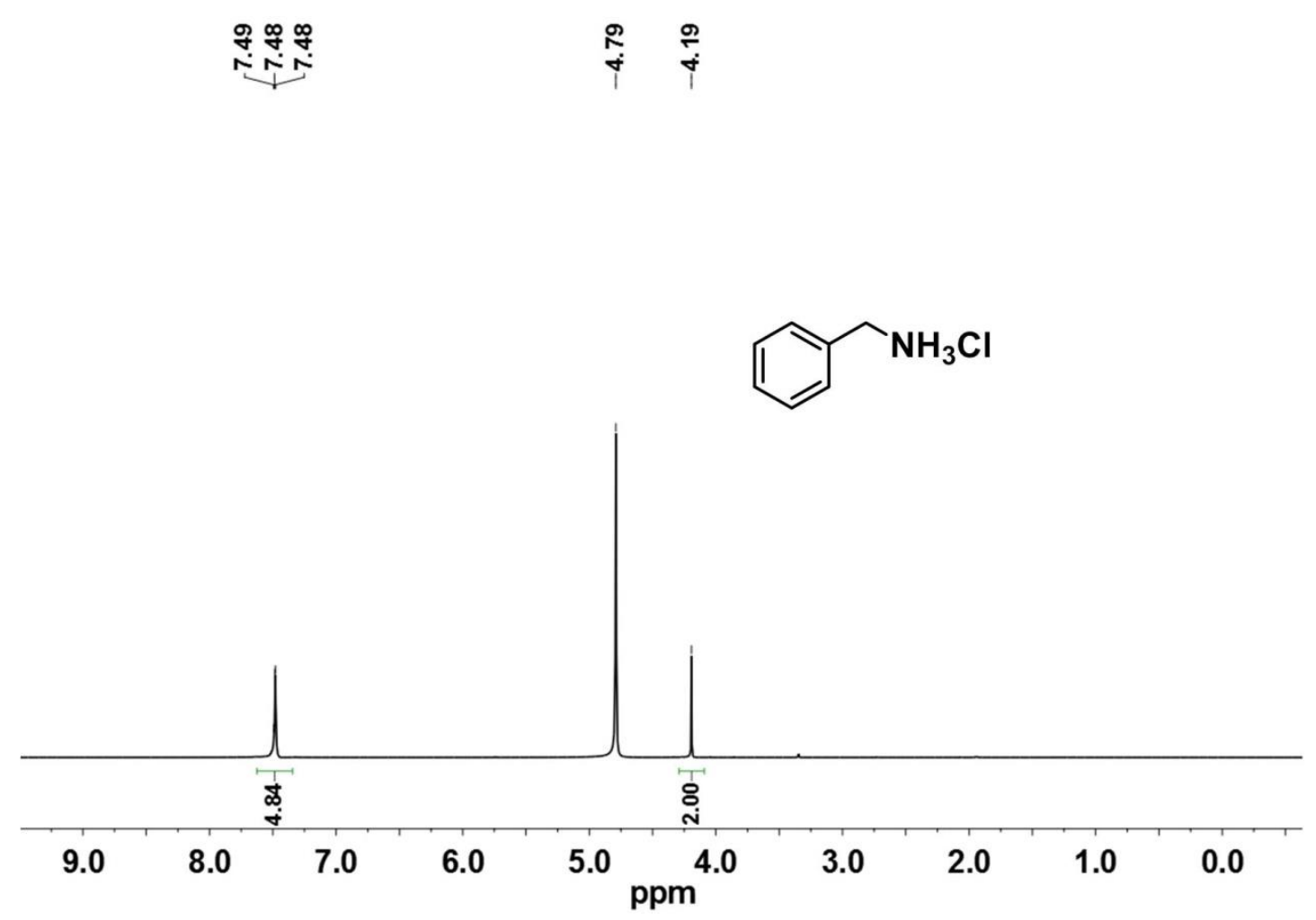

Figure S55. ${ }^{1} \mathrm{H}$ NMR spectrum of benzylamine hydrochloride (4g) in $\mathrm{D}_{2} \mathrm{O}$.

๕.

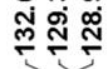

$\stackrel{\bar{y}}{\mathscr{y}}$

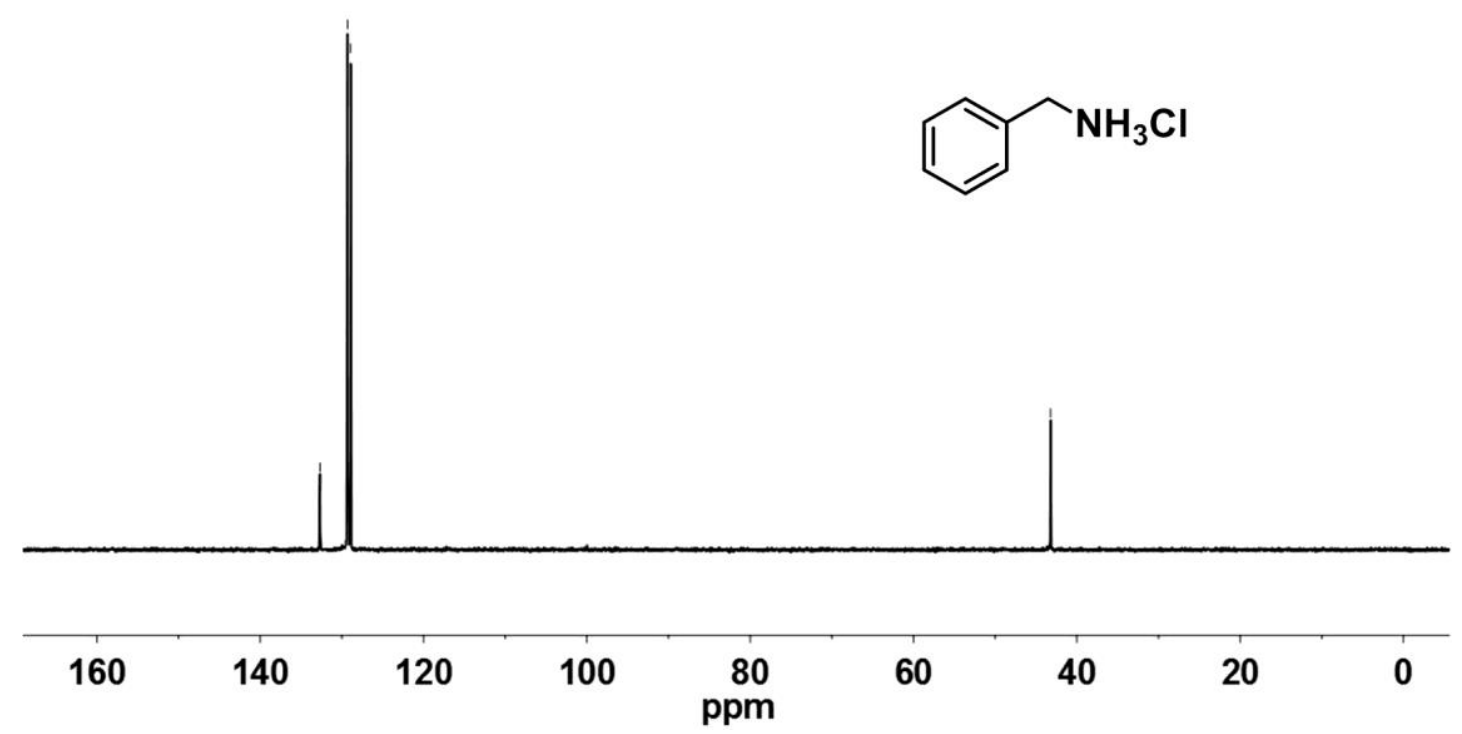

Figure S56. ${ }^{13} \mathrm{C}$ NMR spectrum of benzylamine hydrochloride (4g) in $\mathrm{D}_{2} \mathrm{O}$. 


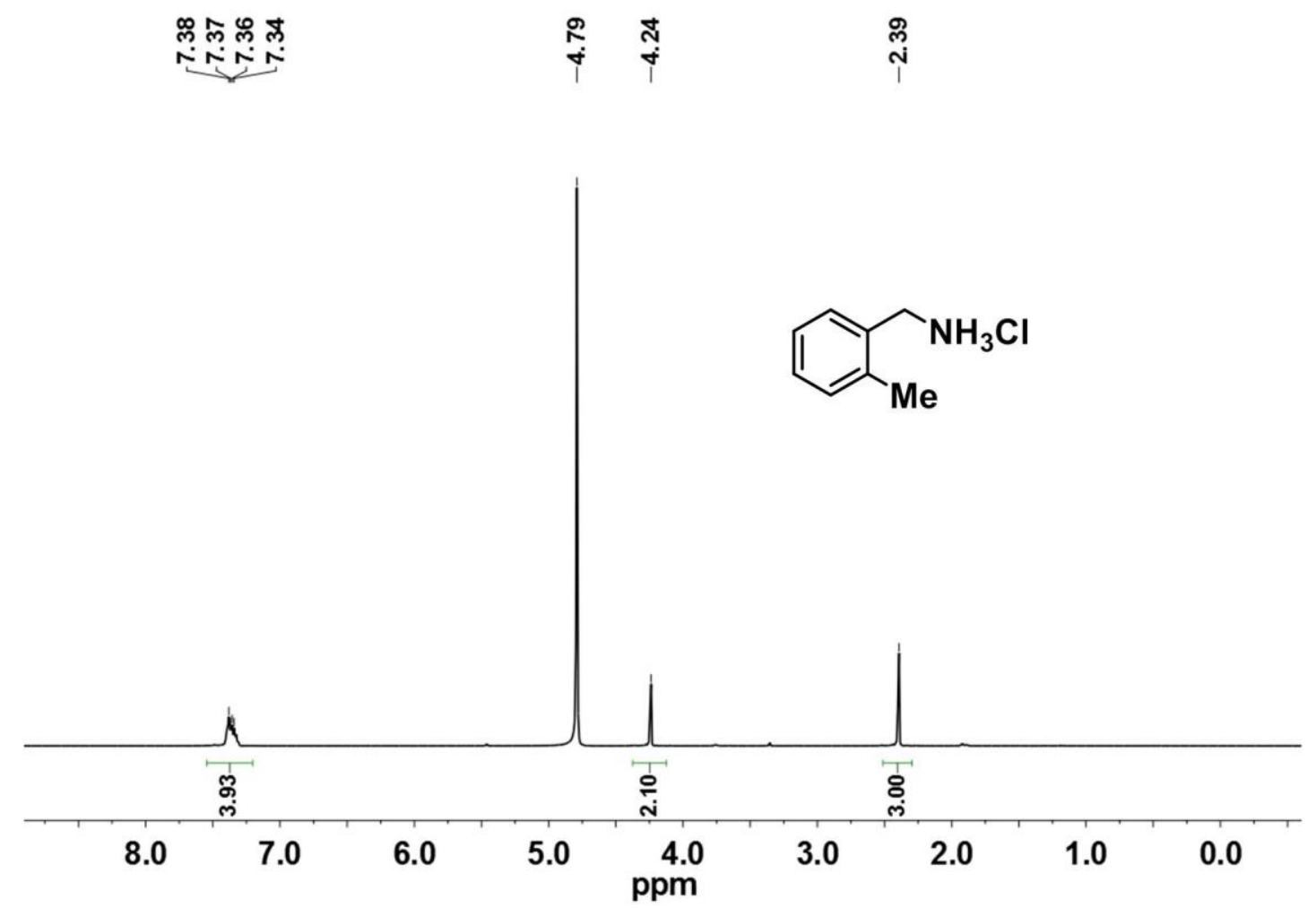

Figure S57. ${ }^{1} \mathrm{H}$ NMR spectrum of 2-methylbenzylamine hydrochloride (4h) in $\mathrm{D}_{2} \mathrm{O}$.

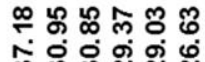

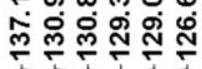

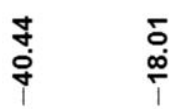

$\overbrace{\mathrm{Me}}^{\mathrm{NH}_{3} \mathrm{Cl}}$

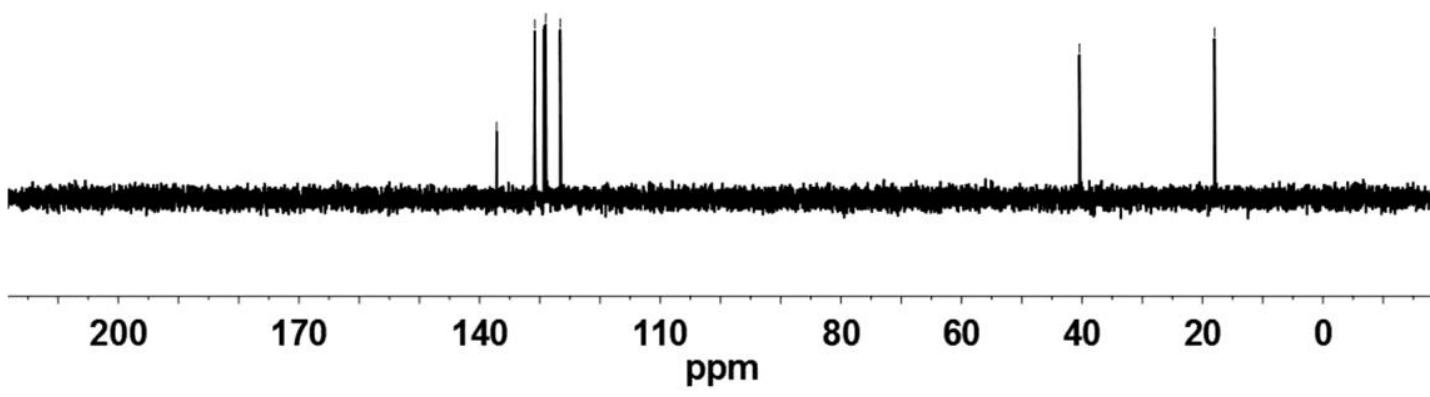

Figure S58. ${ }^{13} \mathrm{C}$ NMR spectrum of 2-methylbenzylamine hydrochloride (4h) in $\mathrm{D}_{2} \mathrm{O}$. 


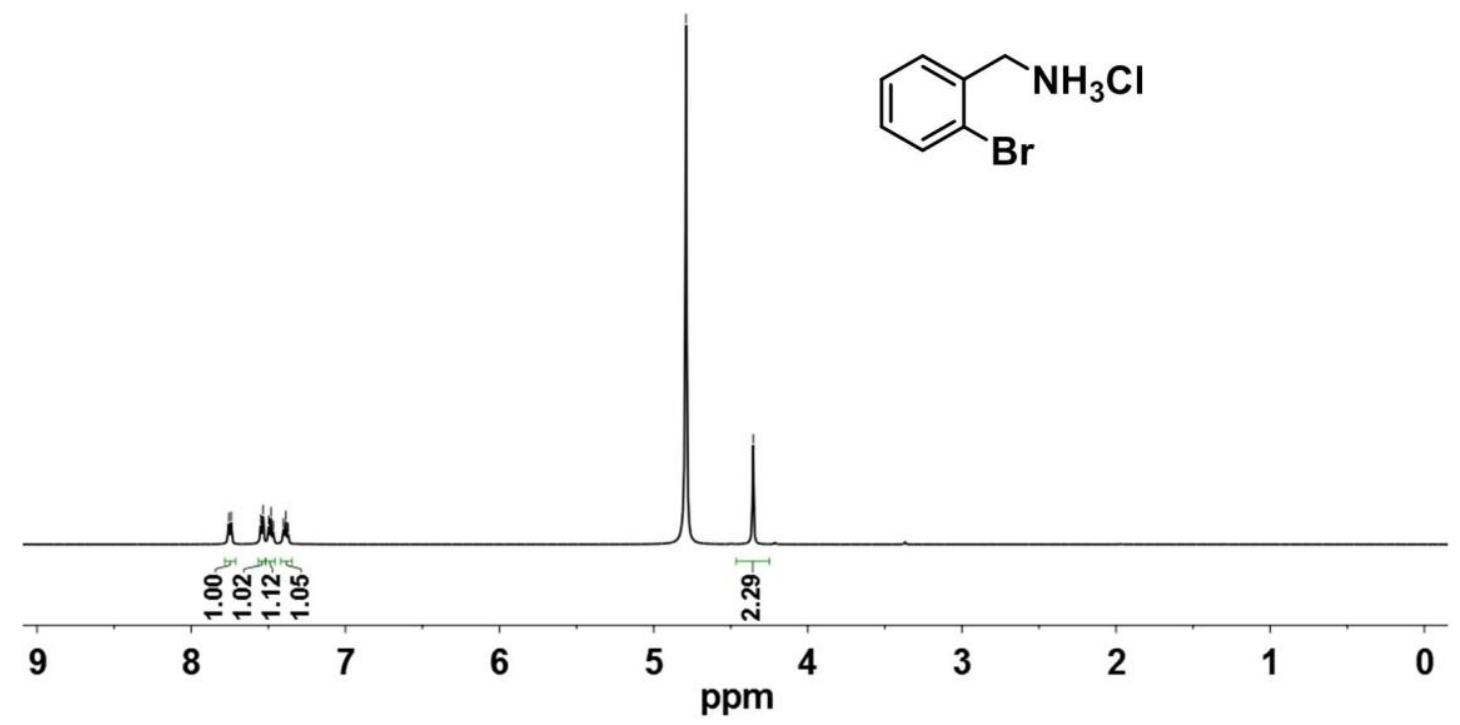

Figure S59. ${ }^{1} \mathrm{H}$ NMR spectrum of 2-bromobenzylamine hydrochloride (4i) in $\mathrm{D}_{2} \mathrm{O}$.

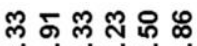

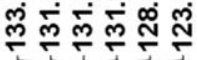

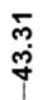<smiles>NCc1ccccc1Br</smiles>

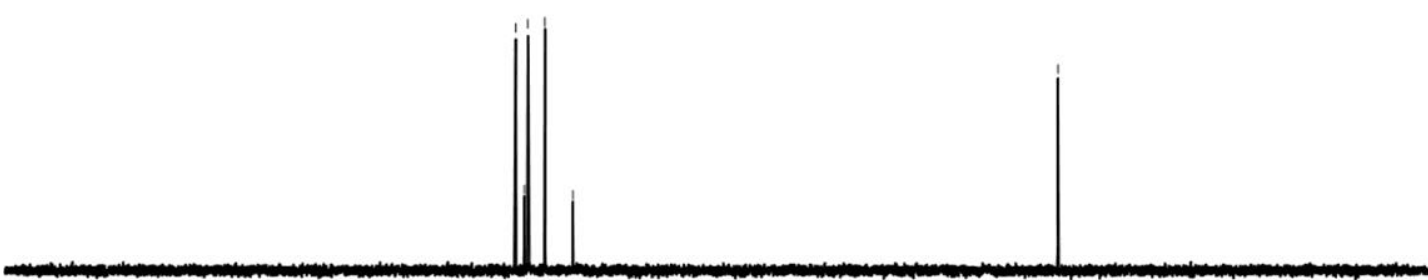

180

160

140120

100

60

$40 \quad 20 \quad 0$

Figure S60. ${ }^{13} \mathrm{C}$ NMR spectrum of 2-bromobenzylamine hydrochloride (4i) in $\mathrm{D}_{2} \mathrm{O}$. 


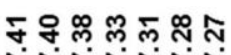

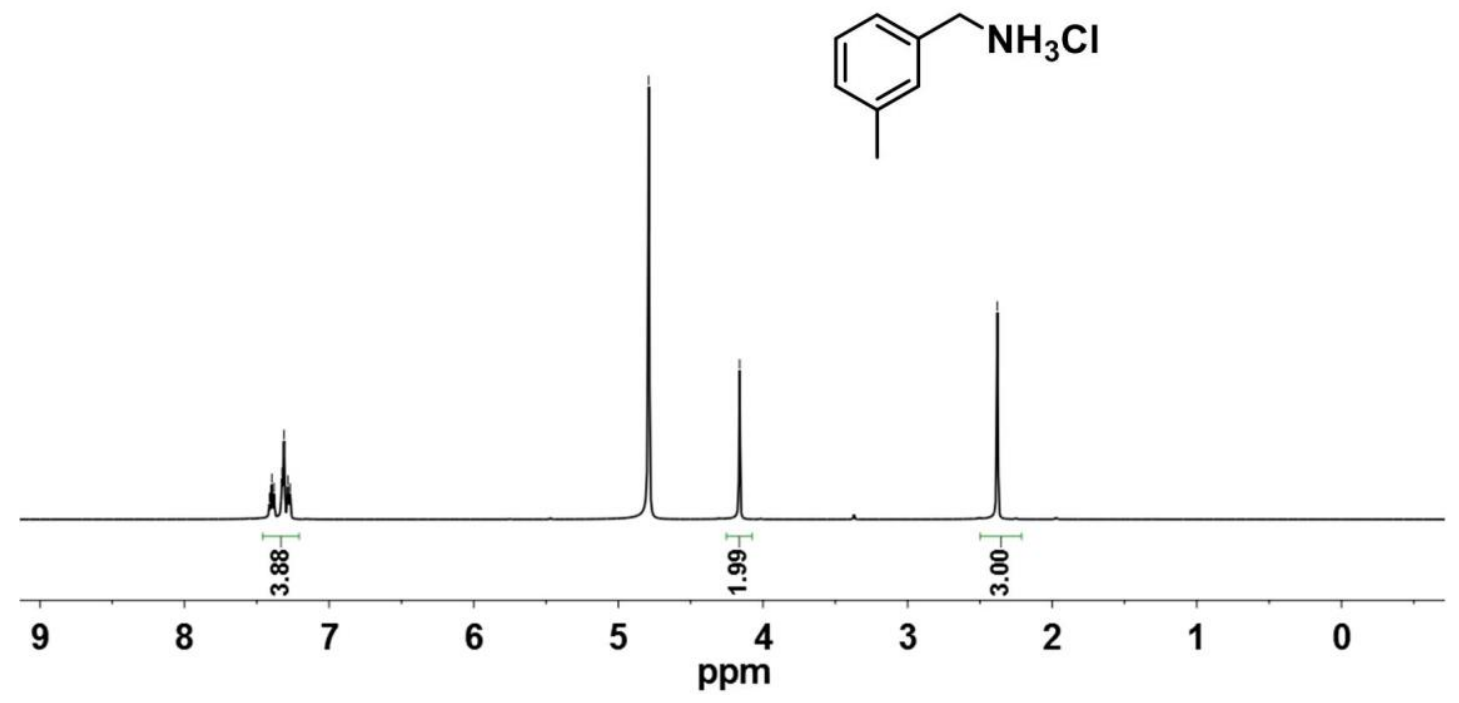

Figure S61. ${ }^{1} \mathrm{H}$ NMR spectrum of 3-methylbenzylamine hydrochloride (4j) in $\mathrm{D}_{2} \mathrm{O}$.

\section{กิ่

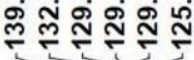
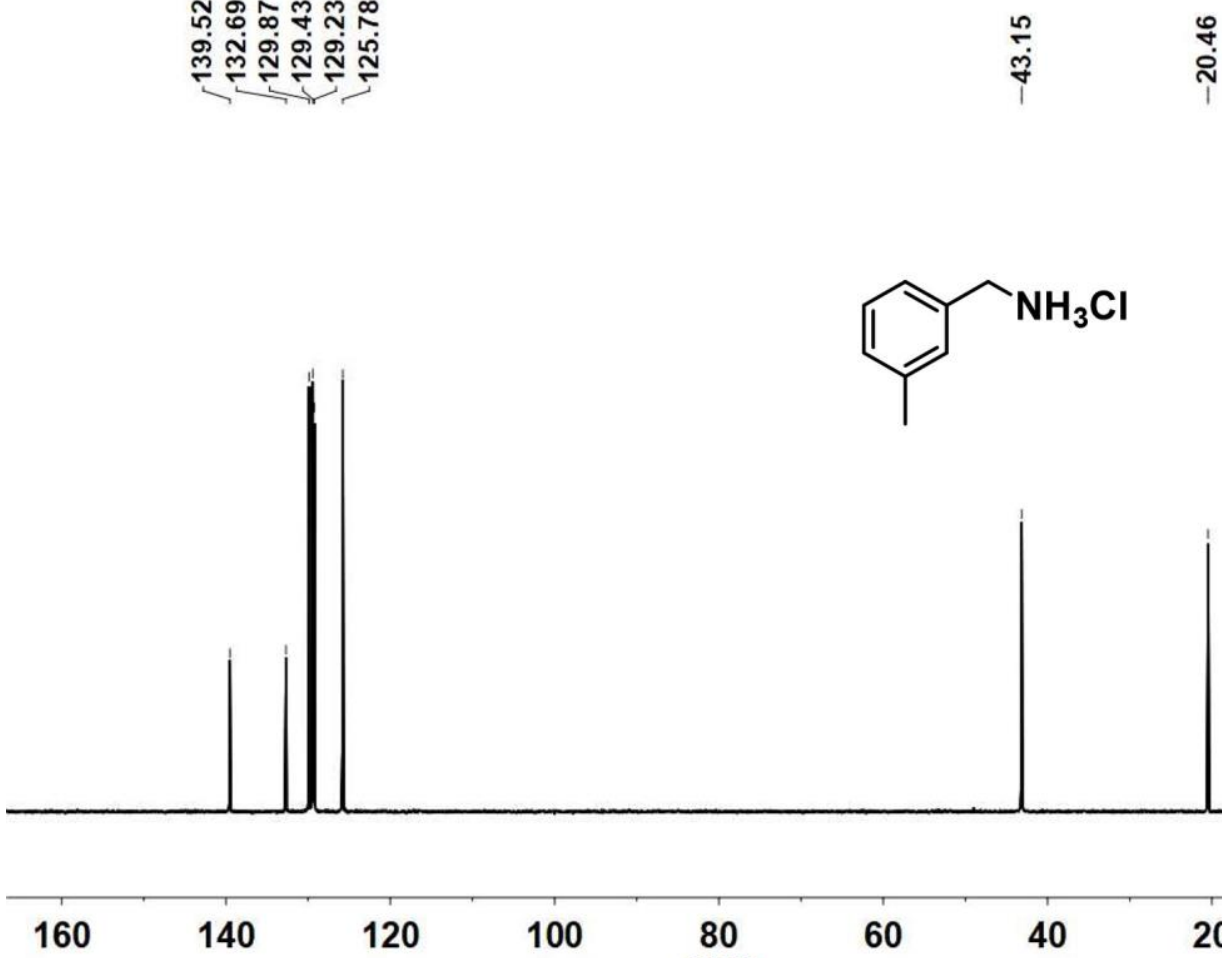<smiles>Cc1cccc(CNCl)c1</smiles>

160 120 100

80
ppm

60 40 20 0

Figure S62. ${ }^{13} \mathrm{C}$ NMR spectrum of 3-methylbenzylamine hydrochloride (4j) in $\mathrm{D}_{2} \mathrm{O}$. 


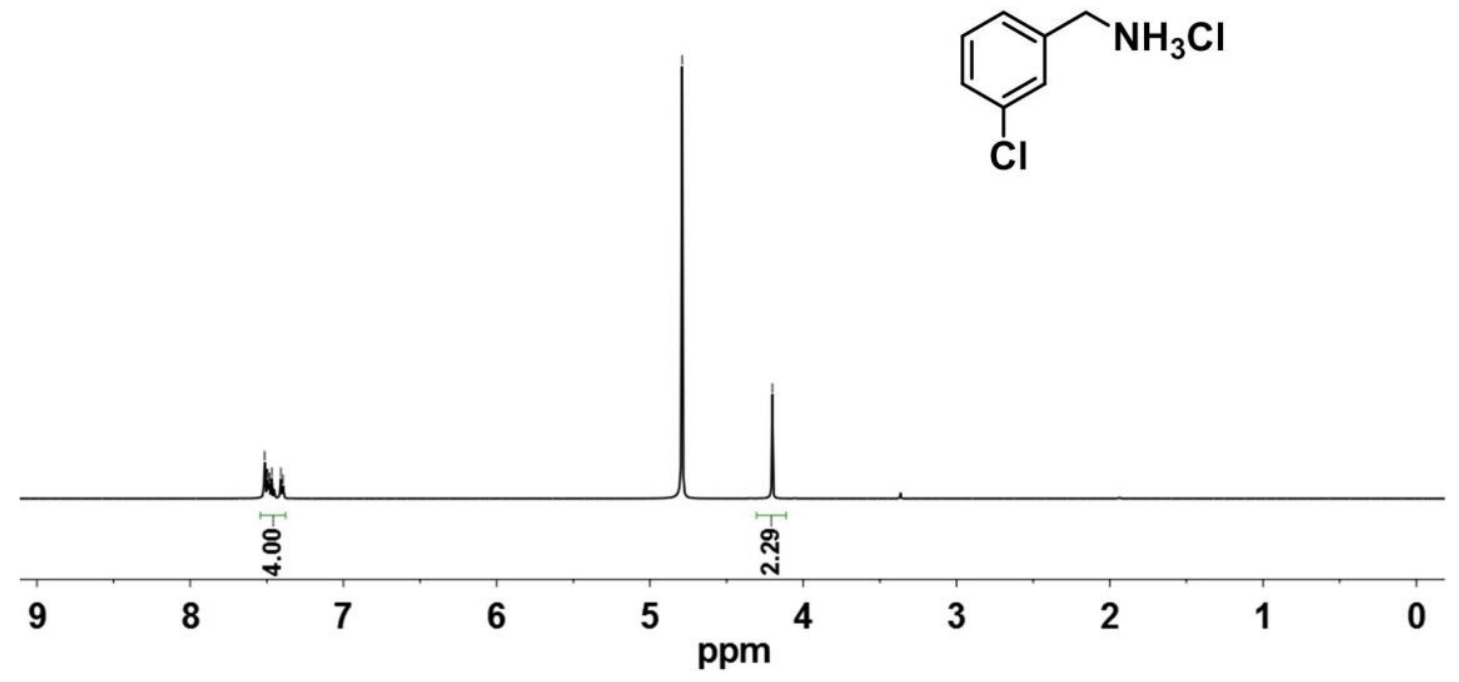

Figure S63. ${ }^{1} \mathrm{H}$ NMR spectrum of 3-chlorobenzylamine hydrochloride (4k) in $\mathrm{D}_{2} \mathrm{O}$.

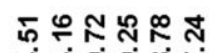

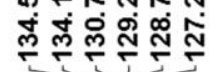<smiles>NCc1cccc(Cl)c1</smiles>

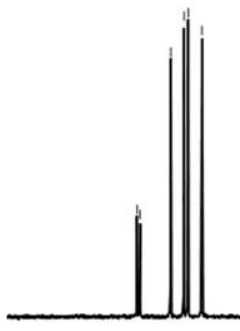

140 120 100 80 ppm 60 40 20 0

Figure S64. ${ }^{13} \mathrm{C}$ NMR spectrum of 3-chlorobenzylamine hydrochloride (4k) in $\mathrm{D}_{2} \mathrm{O}$. 


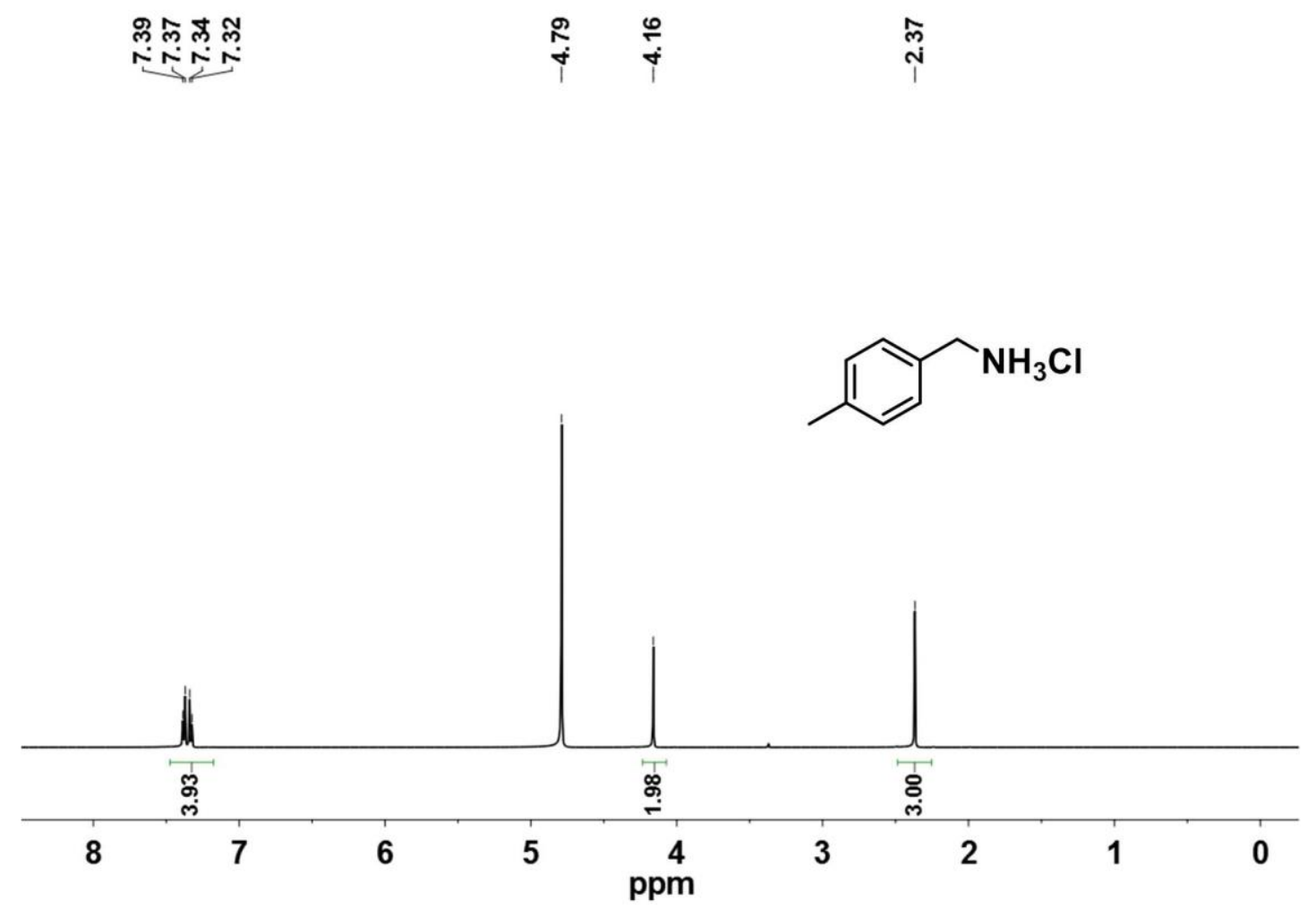

Figure S65. ${ }^{1} \mathrm{H}$ NMR spectrum of 4-methylbenzylamine hydrochloride (4I) in $\mathrm{D}_{2} \mathrm{O}$.

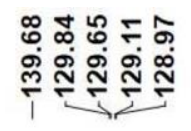

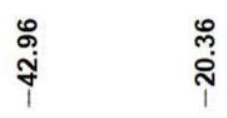

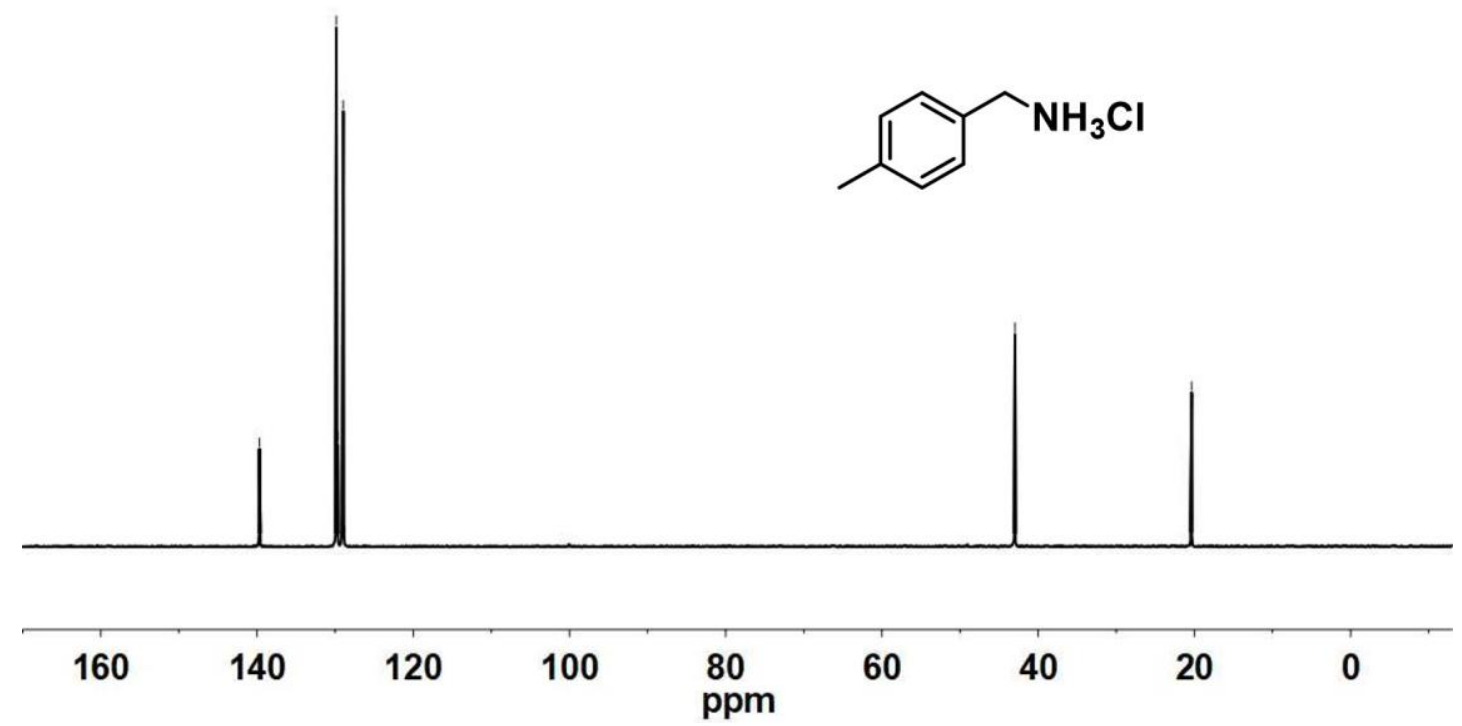

Figure S66. ${ }^{13} \mathrm{C}$ NMR spectrum of 4-methylbenzylamine hydrochloride (4I) in $\mathrm{D}_{2} \mathrm{O}$. 

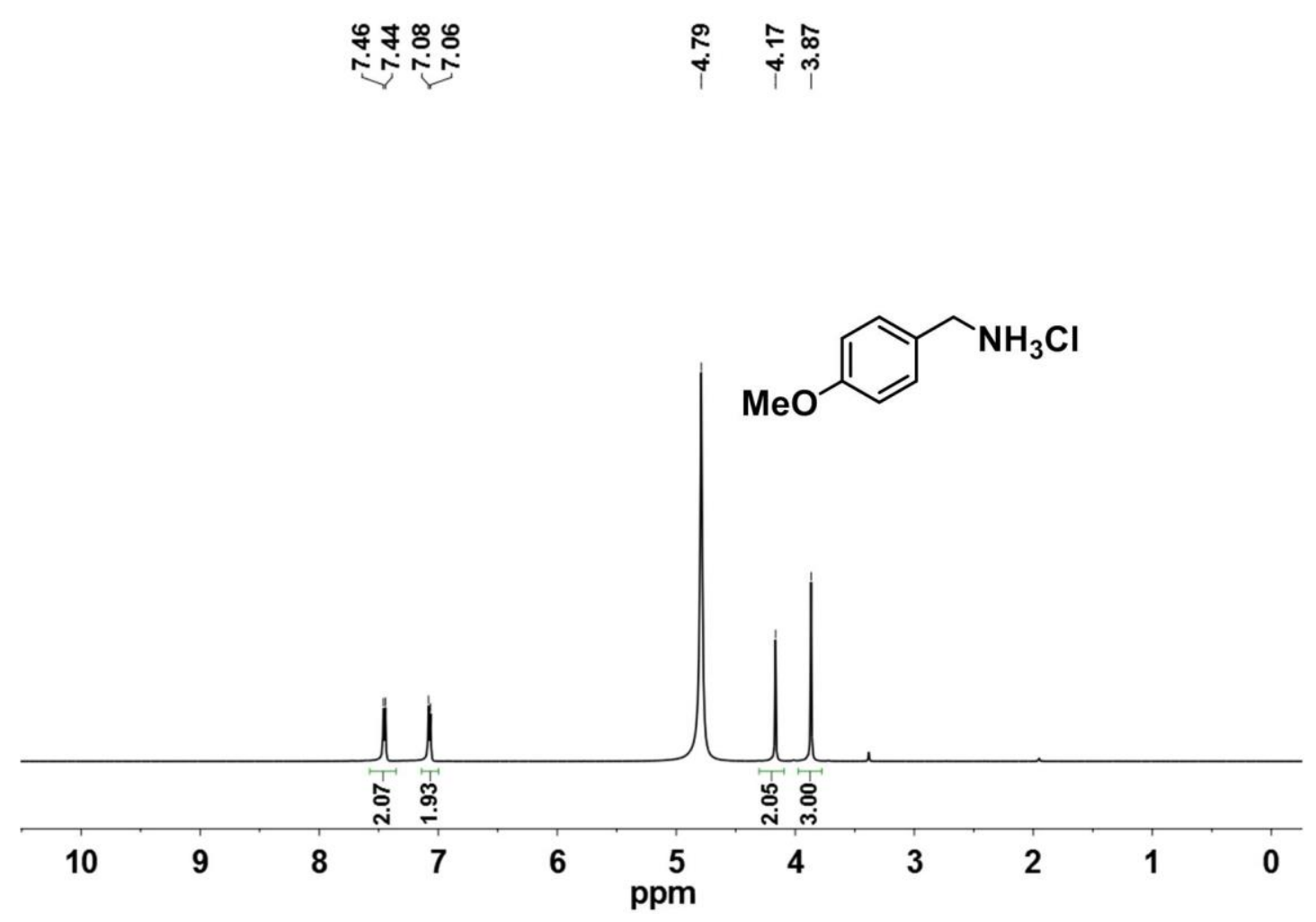

Figure S67. ${ }^{1} \mathrm{H}$ NMR spectrum of 4-methoxybenzylamine hydrochloride (4m) in $\mathrm{D}_{2} \mathrm{O}$.

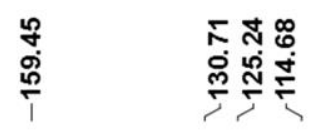

ถึ

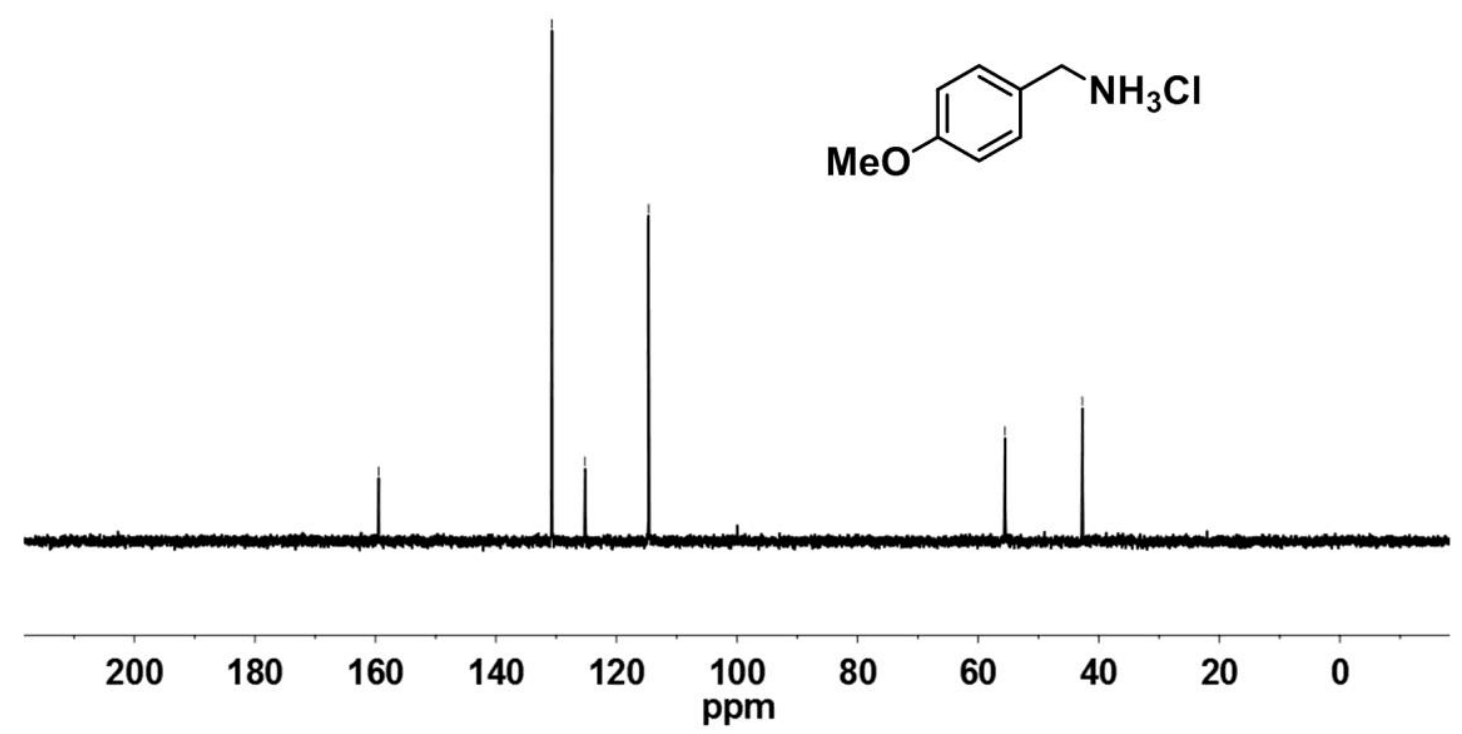

Figure S68. ${ }^{13} \mathrm{C}$ NMR spectrum of 4-methoxybenzylamine hydrochloride (4m) in $\mathrm{D}_{2} \mathrm{O}$. 


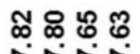

NTN

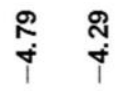

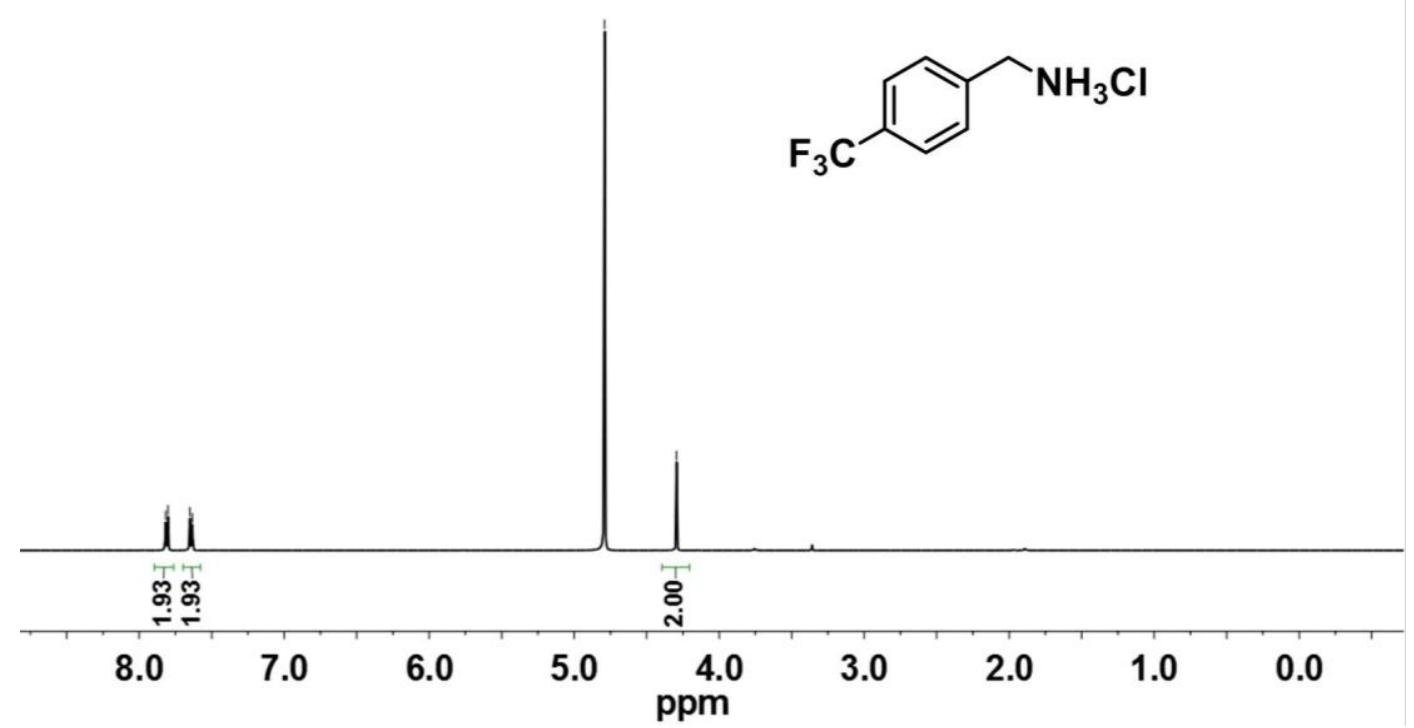

Figure S69. ${ }^{1} \mathrm{H}$ NMR spectrum of 4-trifluoromethylamine hydrochloride (4n) in $\mathrm{D}_{2} \mathrm{O}$.

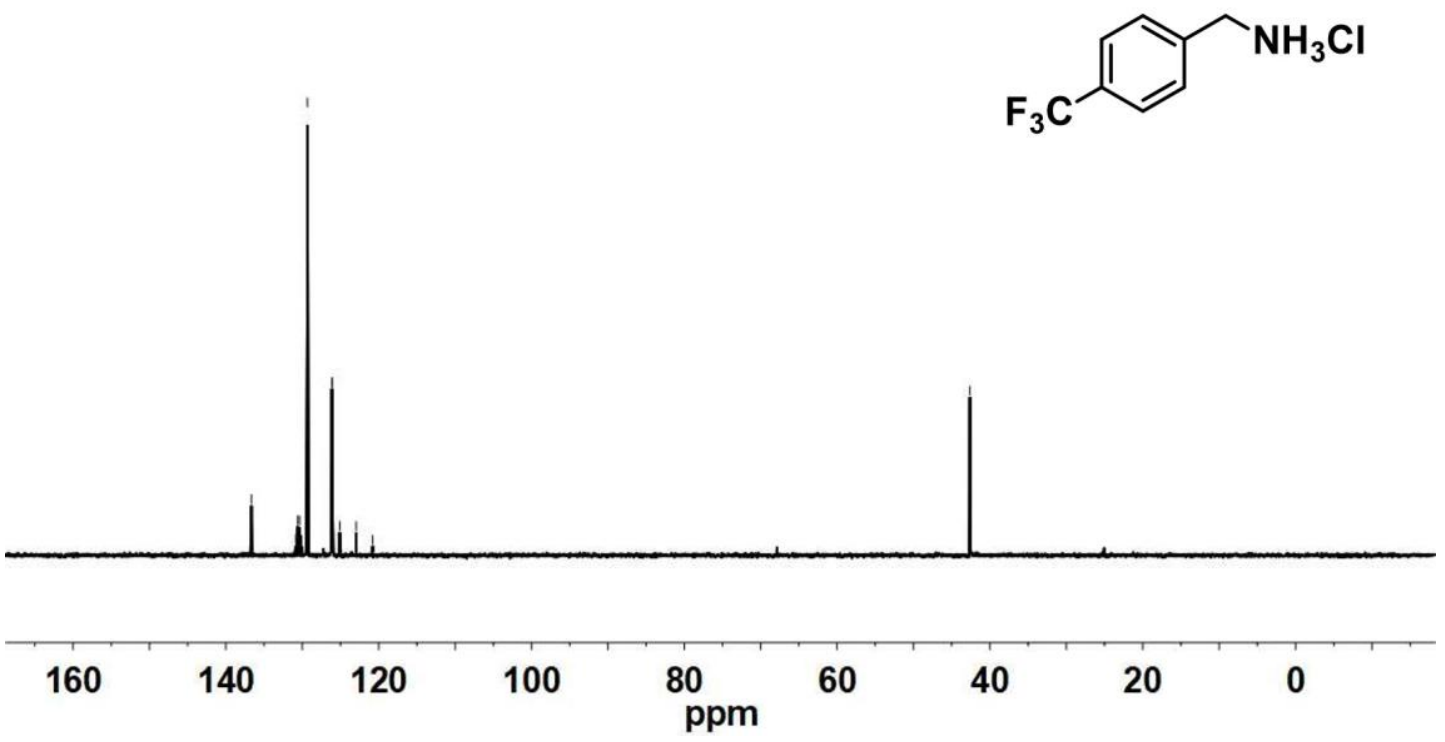

Figure S70. ${ }^{13} \mathrm{C}$ NMR spectrum of 4-trifluoromethylamine hydrochloride (4n) in $\mathrm{D}_{2} \mathrm{O}$. 


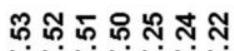

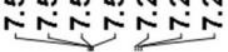

$\stackrel{i}{\stackrel{i}{i}}$

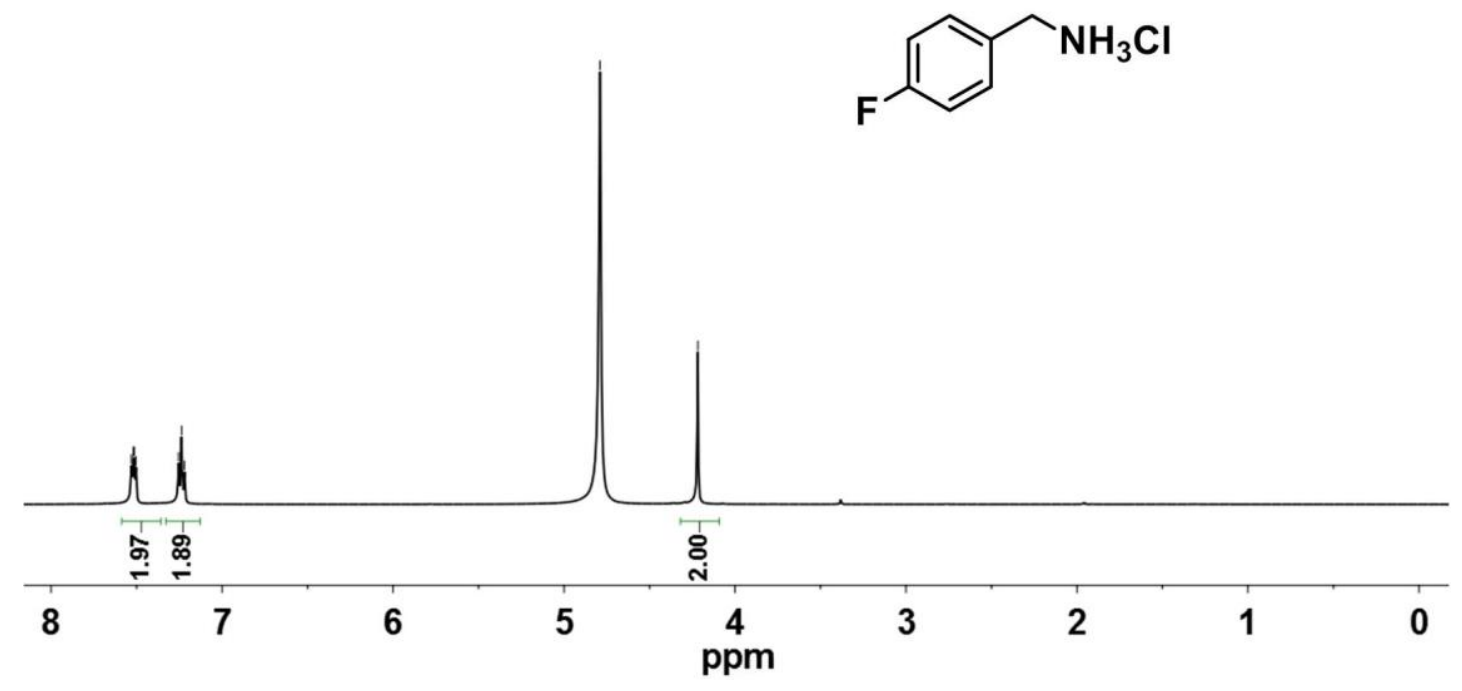

Figure S71. ${ }^{1} \mathrm{H}$ NMR spectrum of 4-fluorobenzylamine hydrochloride (4o) in $\mathrm{D}_{2} \mathrm{O}$.

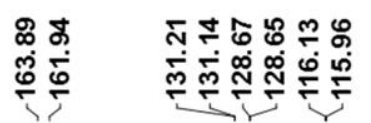

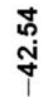<smiles>NCc1ccc(F)cc1</smiles>

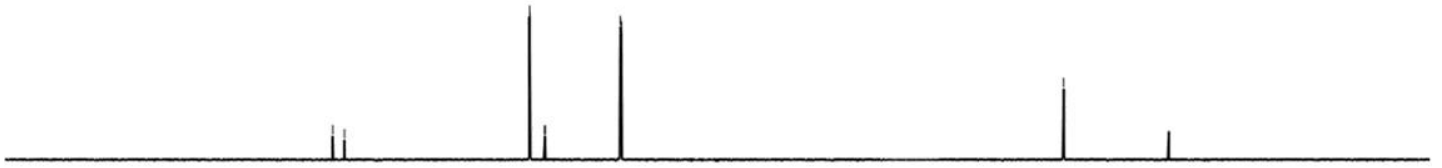

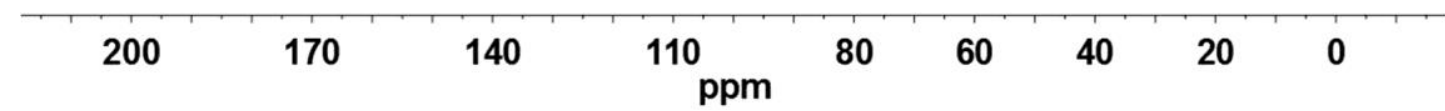

Figure S72. ${ }^{13} \mathrm{C}$ NMR spectrum of 4-fluorobenzylamine hydrochloride (4o) in $\mathrm{D}_{2} \mathrm{O}$. 

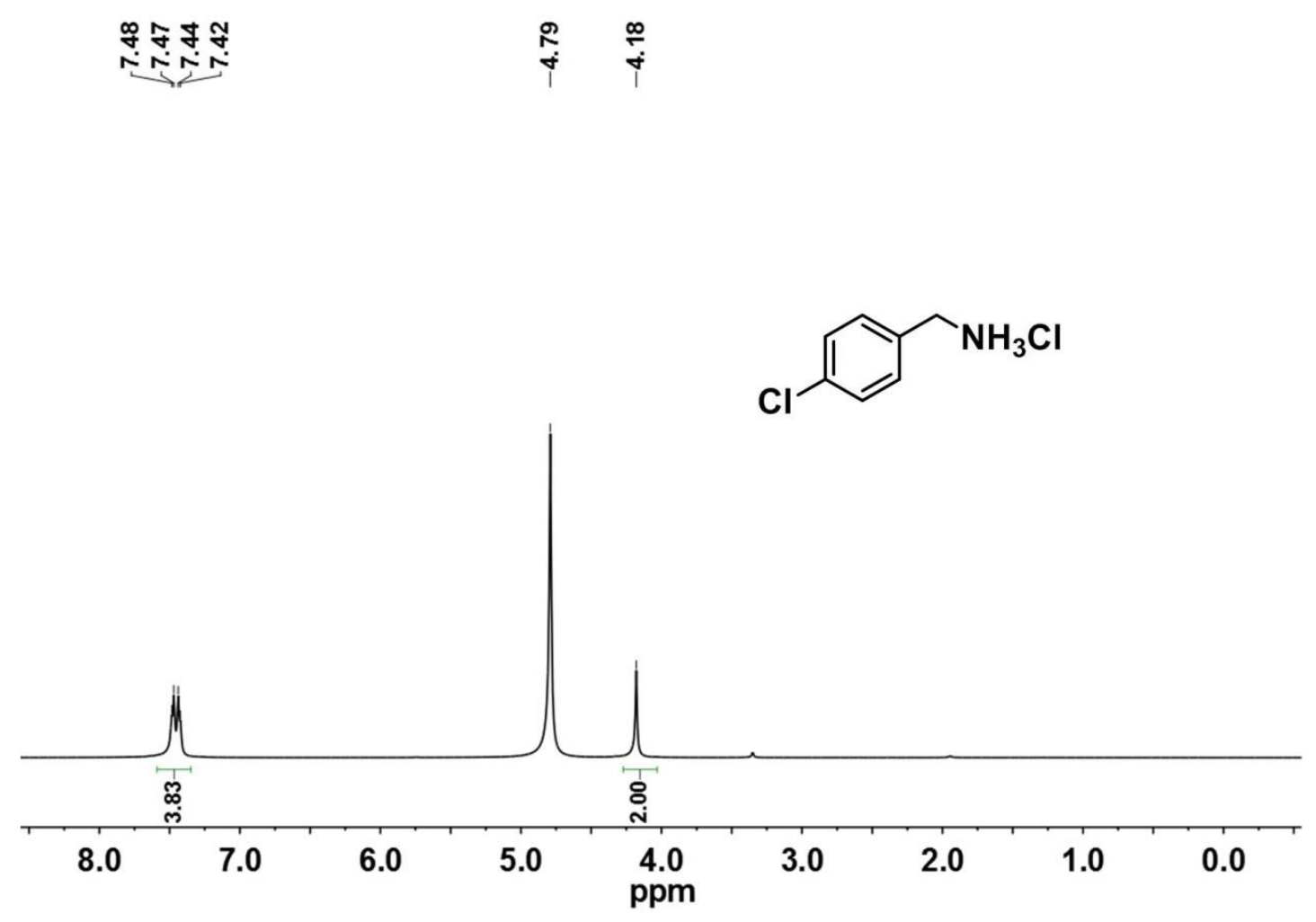

Figure S73. ${ }^{1} \mathrm{H}$ NMR spectrum of 4-chlorobenzylamine hydrochloride (4p) in $\mathrm{D}_{2} \mathrm{O}$.

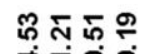

mํํํำ

ণั่

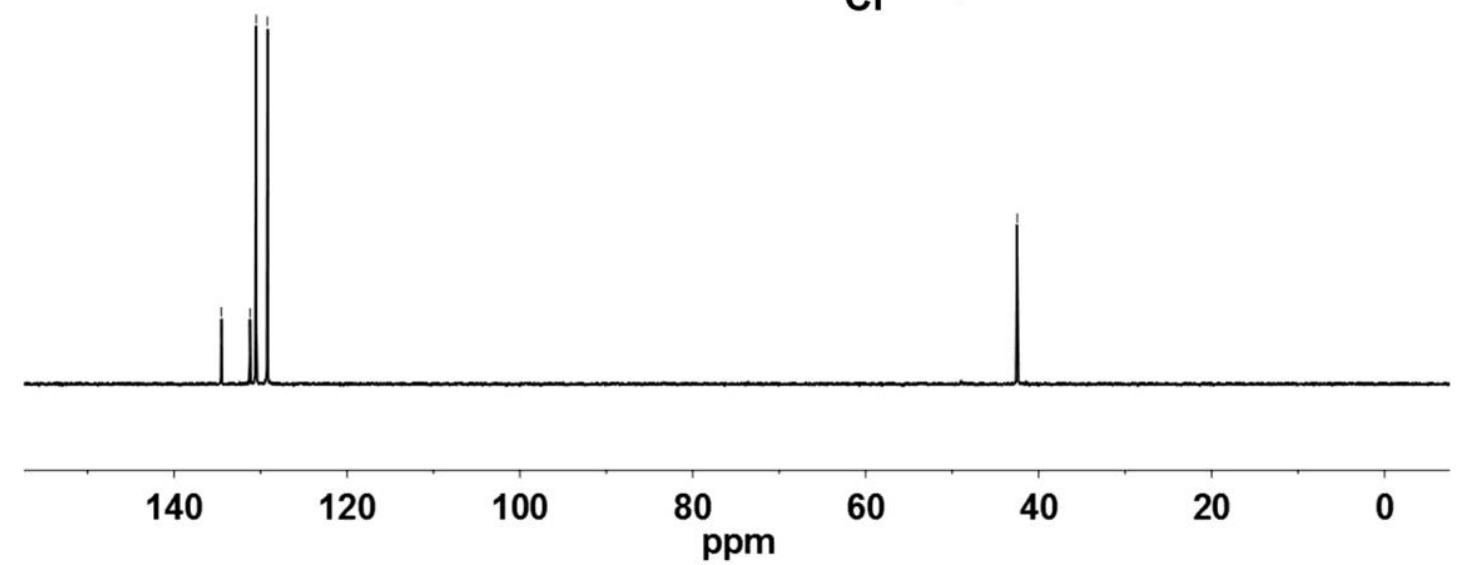<smiles>NCc1ccc(Cl)cc1</smiles>

Figure S74. ${ }^{13} \mathrm{C}$ NMR spectrum of 4-chlorobenzylamine hydrochloride (4p) in $\mathrm{D}_{2} \mathrm{O}$. 
$\mathrm{NH}_{3} \mathrm{COOC}$

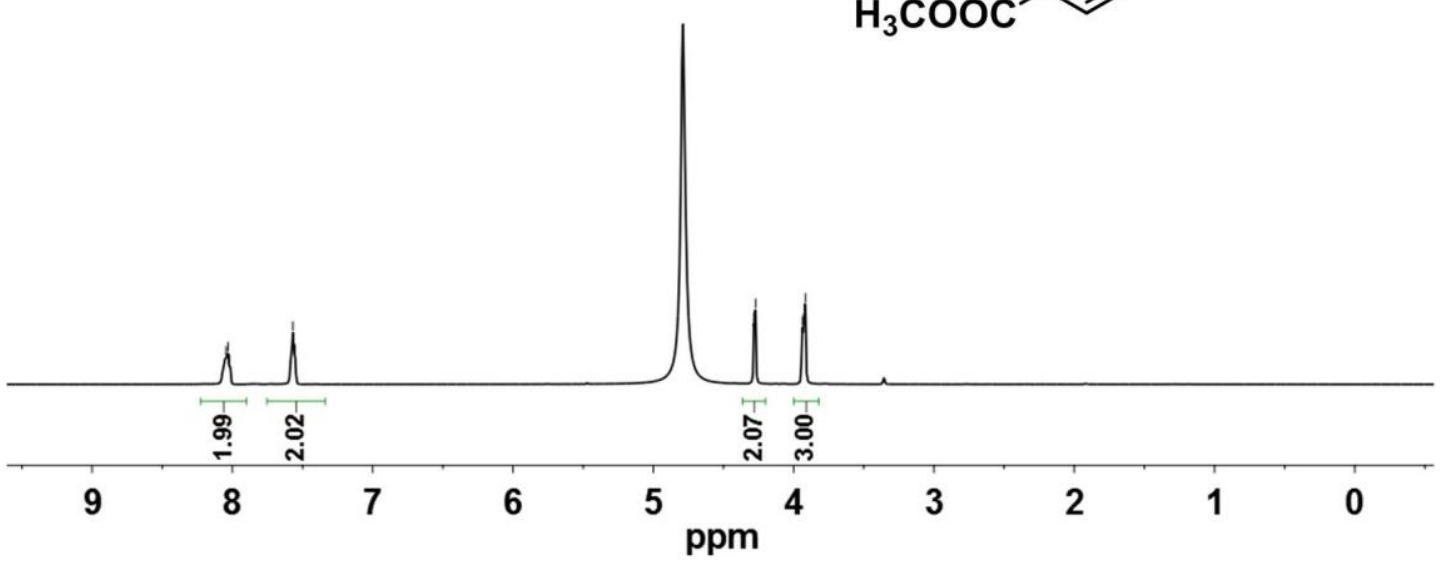

Figure S75. ${ }^{1} \mathrm{H}$ NMR spectrum of 4-(aminomethyl)benzoate hydrochloride (4q) in $\mathrm{D}_{2} \mathrm{O}$.

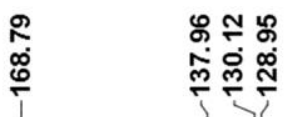

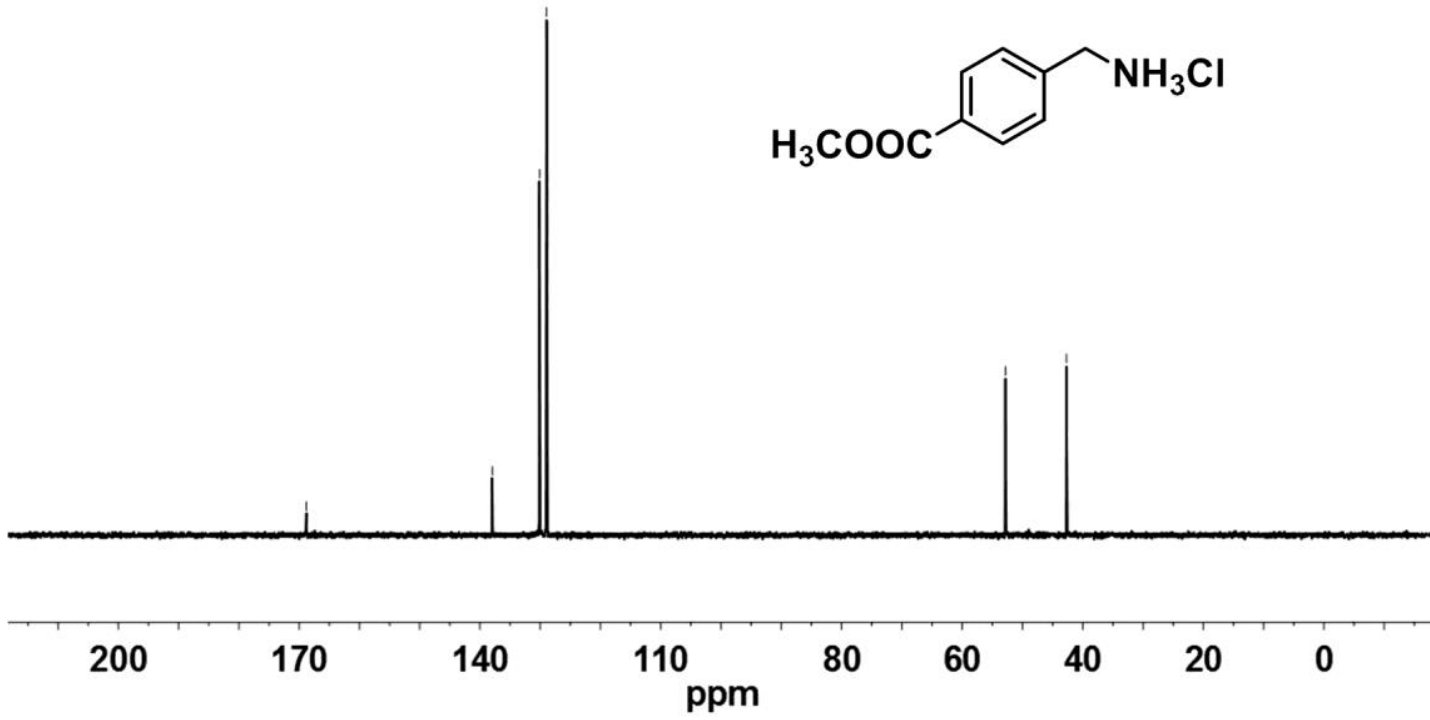

Figure S76. ${ }^{13} \mathrm{C}$ NMR spectrum of 4-(aminomethyl)benzoate hydrochloride (4q) in $\mathrm{D}_{2} \mathrm{O}$. 


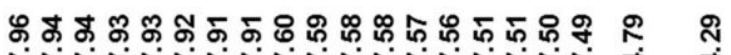

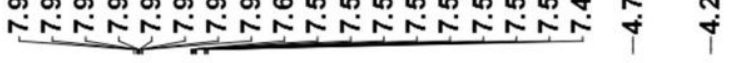<smiles>NCc1ccc2ccccc2c1</smiles>

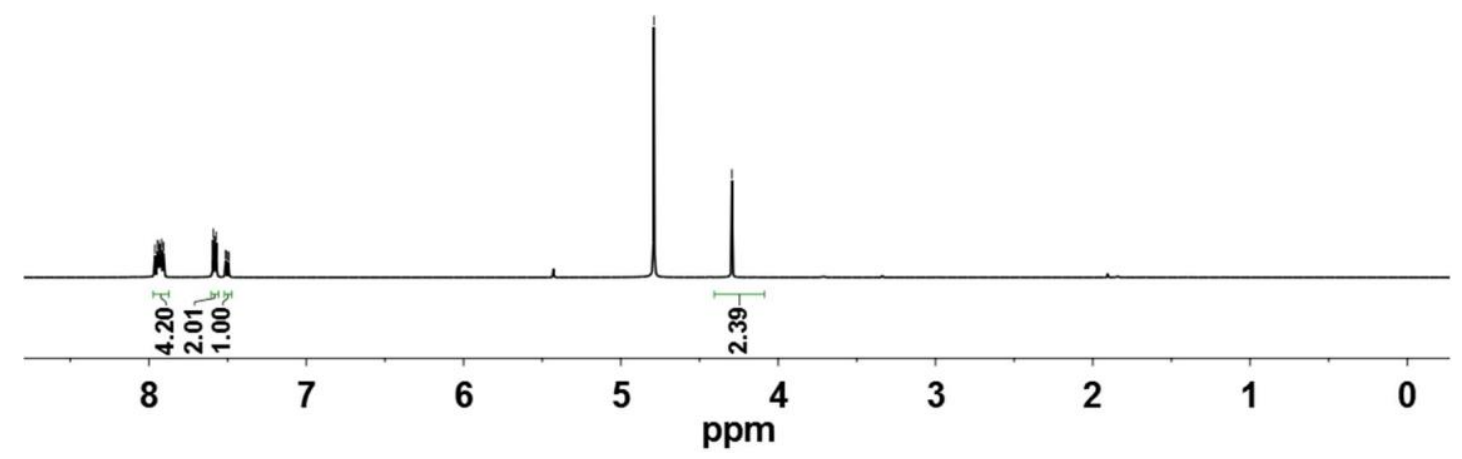

Figure S77. ${ }^{1}$ H NMR spectrum of naphthalen-2-ylmethanammonium chloride (4r) in $\mathrm{D}_{2} \mathrm{O}$.

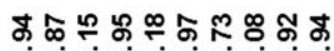

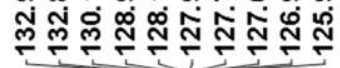<smiles>NCc1ccc2ccccc2c1</smiles>

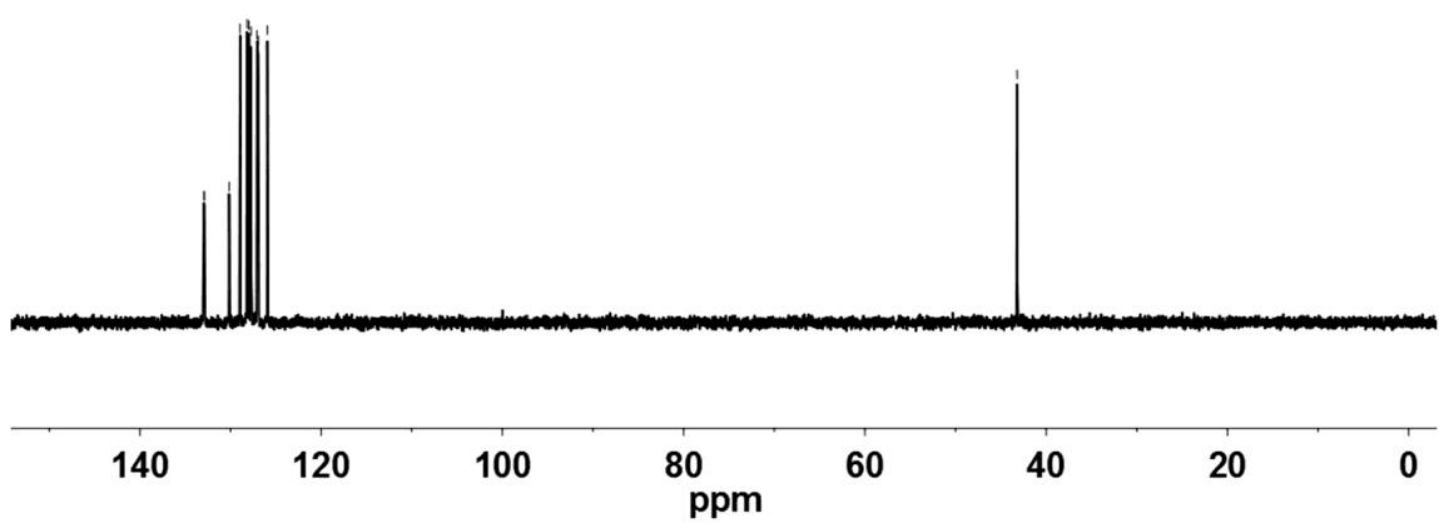

Figure S78. ${ }^{13} \mathrm{C}$ NMR spectrum of naphthalen-2-ylmethanammonium chloride (4r) in $\mathrm{D}_{2} \mathrm{O}$. 


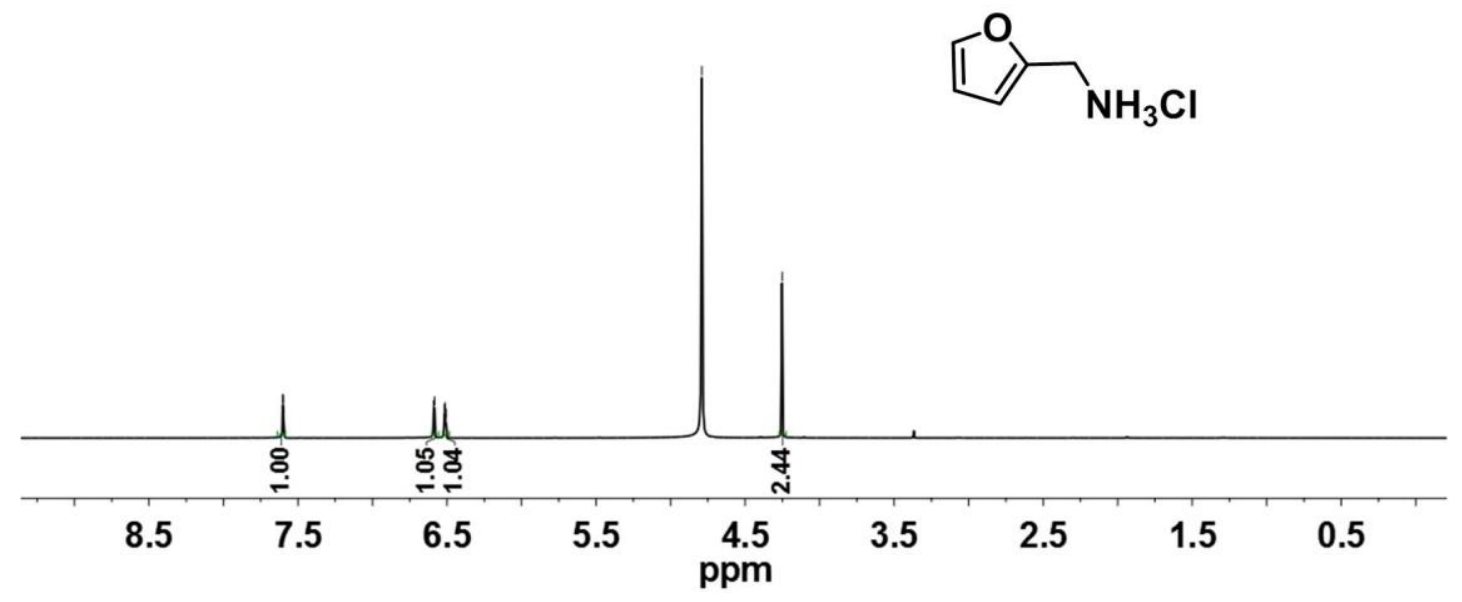

Figure S79. ${ }^{1} \mathrm{H}$ NMR spectrum of furan -2-ylmethanammonium hydrochloride (4s) in $\mathrm{D}_{2} \mathrm{O}$.

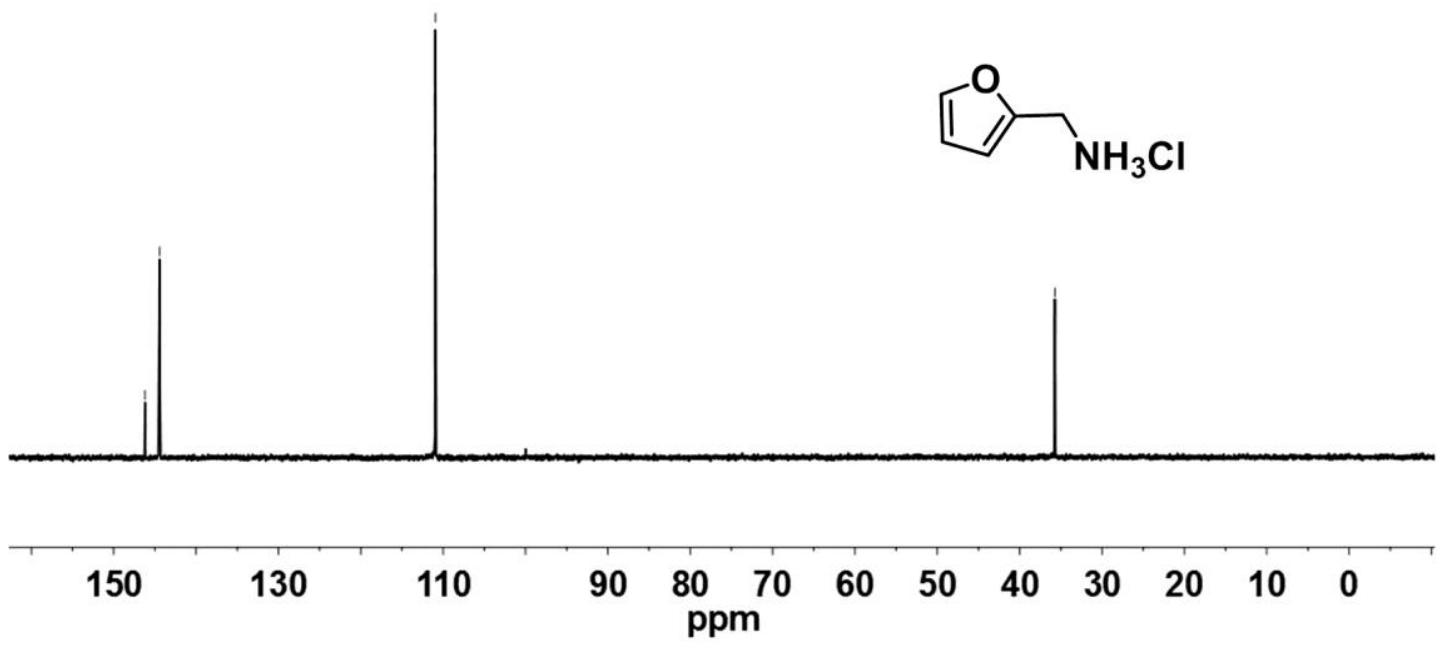

Figure S80. ${ }^{13} \mathrm{C}$ NMR spectrum of furan -2-ylmethanammonium hydrochloride (4s) in $\mathrm{D}_{2} \mathrm{O}$. 


ํํำ

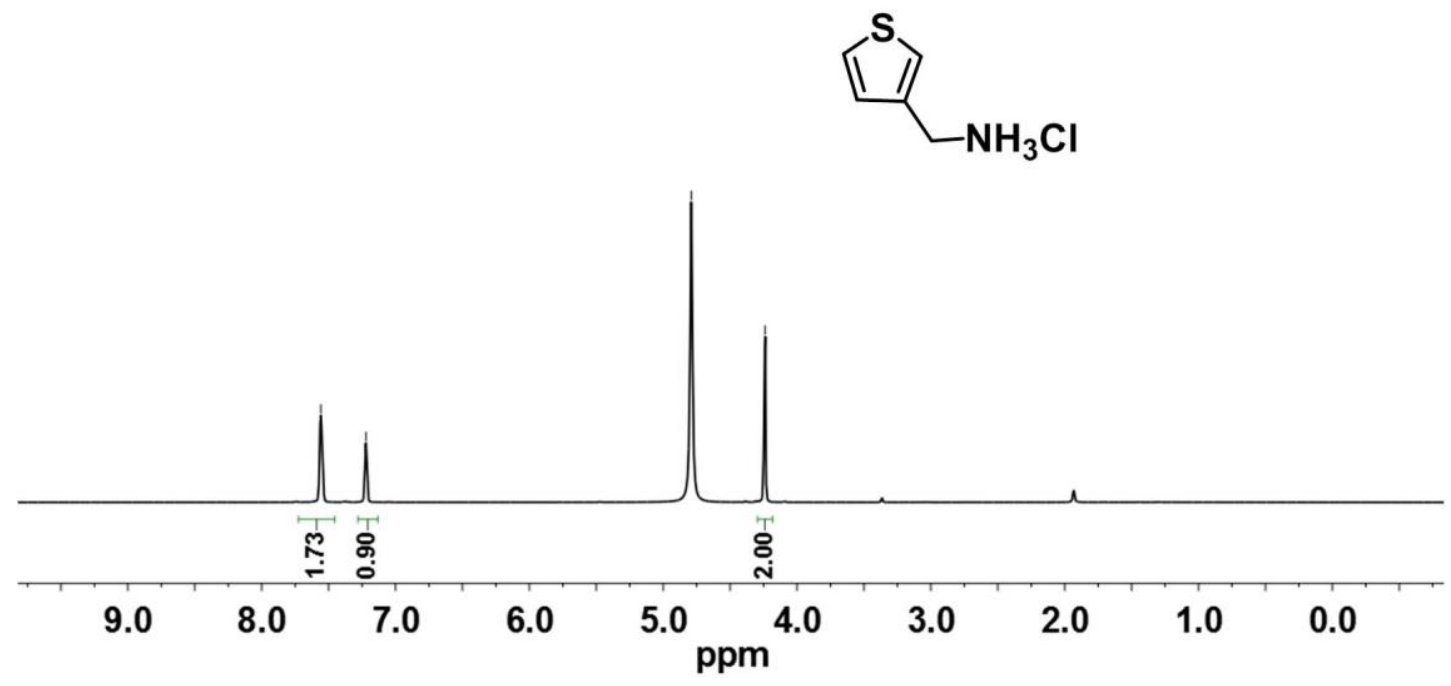

Figure S81. ${ }^{1} \mathrm{H}$ NMR spectrum of thiophen-3-ylmethanammonium hydrochloride (4t) in $\mathrm{D}_{2} \mathrm{O}$.

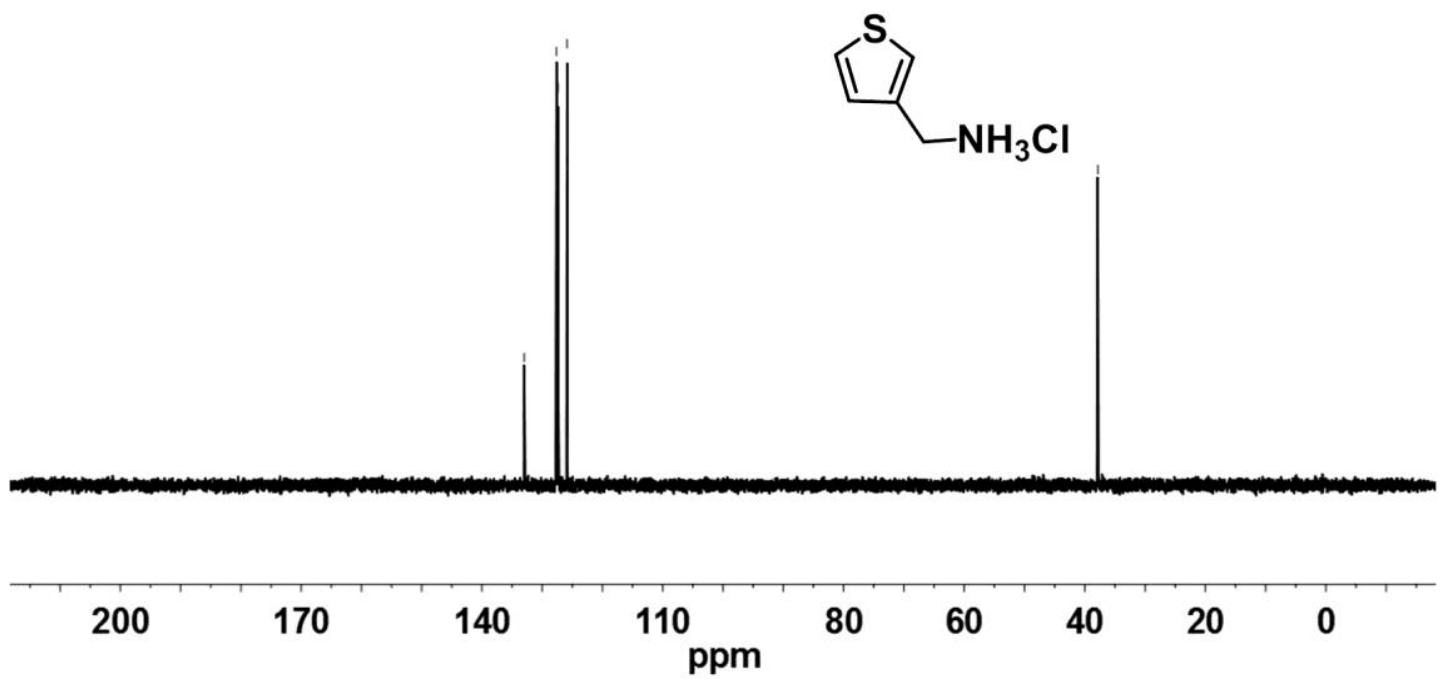

Figure S82. ${ }^{13} \mathrm{C}$ NMR spectrum of thiophen-3-ylmethanammonium hydrochloride (4t) in $\mathrm{D}_{2} \mathrm{O}$. 


\section{X-ray crystal structure analysis}

Table S5. Crystal data and structure refinement of complexes

\begin{tabular}{|c|c|c|c|c|}
\hline & $\mathbf{1}(\mathrm{CO})_{2}$ & $\mathbf{1}(\mathrm{NCMe})_{3}\left(\mathrm{BF}_{4}\right)_{2}$ & $\mathbf{1}\left(\eta^{2}-\mathrm{NCMe}\right)$ & $\mathbf{1 H}\left(\mathrm{BH}_{2}\right)$ \\
\hline $\begin{array}{l}\text { Empirical } \\
\text { formula }\end{array}$ & $\mathrm{C}_{30} \mathrm{H}_{29} \mathrm{MoO}_{2} \mathrm{PS}$ & $\mathrm{C}_{34} \mathrm{H}_{38} \mathrm{~B}_{2} \mathrm{~F}_{8} \mathrm{MoN}_{3} \mathrm{PS}$ & $\mathrm{C}_{30} \mathrm{H}_{32} \mathrm{MoNPS}$ & $\mathrm{C}_{28} \mathrm{H}_{32} \mathrm{BMoPS}$ \\
\hline Formula weight & 580.53 & 821.26 & 565.57 & 538.31 \\
\hline Temperature/K & $172.99(10)$ & 173.0 & $172.99(10)$ & $173.00(10)$ \\
\hline Crystal system & monoclinic & triclinic & monoclinic & monoclinic \\
\hline Space group & $C 2 / \mathrm{c}$ & $P-1$ & $P 2_{1} / \mathrm{n}$ & $P 2{ }_{1} / \mathrm{n}$ \\
\hline $\mathrm{a} / \AA$ & $19.389(5)$ & $11.1302(5)$ & $11.1248(2)$ & $10.8462(2)$ \\
\hline $\mathrm{b} / \AA$ & $15.694(4)$ & $13.2104(5)$ & $18.1970(3)$ & $16.9076(3)$ \\
\hline $\mathrm{c} / \AA$ & $17.490(4)$ & $13.9442(5)$ & $12.9432(2)$ & $14.8894(3)$ \\
\hline$\alpha /{ }^{\circ}$ & 90 & $75.533(3)$ & 90 & 90 \\
\hline$\beta /{ }^{\circ}$ & $101.224(2)$ & $73.017(4)$ & $90.632(2)$ & $105.260(2)$ \\
\hline$\gamma /{ }^{\circ}$ & 90 & $67.252(4)$ & 90 & 90 \\
\hline Volume $/ \AA^{3}$ & $5220(2)$ & $1786.43(14)$ & $2620.04(8)$ & $2634.19(9)$ \\
\hline $\mathrm{Z}$ & 8 & 2 & 4 & 4 \\
\hline$\rho_{\text {calcg }} / \mathrm{cm}^{3}$ & 1.4772 & 1.527 & 1.4337 & 1.357 \\
\hline$\mu / \mathrm{mm}^{-1}$ & 0.667 & 0.542 & 5.548 & 5.471 \\
\hline $\mathrm{F}(000)$ & 2372.8 & 836.0 & 1172.7 & 1112.0 \\
\hline Radiation & $\begin{array}{l}\text { synchrotron }(\lambda= \\
0.71)\end{array}$ & $\operatorname{Mo} \mathrm{K} \alpha(\lambda=0.710)$ & $\begin{array}{lr}\mathrm{Cu} & \mathrm{K} \alpha \\
1.54184) & \end{array}$ & $\mathrm{Cu} \mathrm{K} \alpha(\lambda=1.54184)$ \\
\hline $\begin{array}{l}2 \Theta \text { range for data } \\
\text { collection } /{ }^{\circ}\end{array}$ & 3.36 to 50.64 & 3.088 to 49.998 & 8.38 to 134.16 & 8.076 to 134.11 \\
\hline Index ranges & $\begin{array}{l}-25 \leq \mathrm{h} \leq 25,-20 \leq \\
\mathrm{k} \leq 19,-22 \leq 1 \leq 22\end{array}$ & $\begin{array}{l}-11 \leq \mathrm{h} \leq 13,-11 \leq \mathrm{k} \leq \\
15,-15 \leq 1 \leq 16\end{array}$ & $\begin{array}{l}-14 \leq \mathrm{h} \leq 12,-9 \leq \\
\mathrm{k} \leq 22,-16 \leq 1 \leq 15\end{array}$ & $\begin{array}{l}-12 \leq \mathrm{h} \leq 12,-20 \leq \mathrm{k} \\
\leq 20,-17 \leq 1 \leq 14\end{array}$ \\
\hline $\begin{array}{l}\text { Reflections } \\
\text { collected }\end{array}$ & 18443 & 11933 & 15568 & 16030 \\
\hline $\begin{array}{l}\text { Independent } \\
\text { reflections }\end{array}$ & $\begin{array}{l}4771 \quad\left[\mathrm{R}_{\text {int }}=\right. \\
0.0487, \quad \mathrm{R}_{\text {sigma }}= \\
0.0595]\end{array}$ & $\begin{array}{l}6298 \quad\left[R_{\text {int }}=0.0399,\right. \\
\left.\mathrm{R}_{\text {sigma }}=0.0488\right]\end{array}$ & $\begin{array}{l}4632 \quad\left[R_{\text {int }}=\right. \\
0.0579, \quad R_{\text {sigma }}= \\
0.0594]\end{array}$ & $\begin{array}{l}4648\left[R_{\text {int }}=0.0475,\right. \\
\left.R_{\text {sigma }}=0.0443\right]\end{array}$ \\
\hline $\begin{array}{l}\text { Data/restraints } / \mathrm{p} \\
\text { arameters }\end{array}$ & $4771 / 0 / 321$ & $6298 / 1 / 459$ & $4632 / 0 / 313$ & $4648 / 0 / 306$ \\
\hline $\begin{array}{l}\text { Goodness-of-fit } \\
\text { on } \mathrm{F}^{2}\end{array}$ & 1.055 & 1.072 & 1.028 & 1.048 \\
\hline $\begin{array}{l}\text { Final } R \text { indexes } \\
{[\mathrm{I}>=2 \sigma(\mathrm{I})]}\end{array}$ & $\begin{array}{l}\mathrm{R}_{1}=0.0351 \\
\mathrm{wR}_{2}=0.0725\end{array}$ & $\begin{array}{l}\mathrm{R}_{1}=0.0438 \\
\mathrm{wR}_{2}=0.1065\end{array}$ & $\begin{array}{l}\mathrm{R}_{1}=0.0433 \\
\mathrm{wR}_{2}=0.1017\end{array}$ & $\begin{array}{l}\mathrm{R}_{1}=0.0364 \quad \mathrm{wR}_{2}= \\
0.0933\end{array}$ \\
\hline $\begin{array}{l}\text { Final } \mathrm{R} \text { indexes } \\
\text { [all data] }\end{array}$ & $\begin{array}{l}\mathrm{R}_{1}=0.0517 \\
\mathrm{wR}_{2}=0.0822\end{array}$ & $\begin{array}{l}\mathrm{R}_{1}=0.0519 \\
\mathrm{wR}_{2}=0.1135\end{array}$ & $\begin{array}{l}\mathrm{R}_{1}=0.0585 \\
\mathrm{wR}_{2}=0.1163\end{array}$ & $\begin{array}{l}\mathrm{R}_{1}=0.0463, \quad \mathrm{wR}_{2}= \\
0.1029\end{array}$ \\
\hline $\begin{array}{l}\text { Largest diff. } \\
\text { peak/hole / e } \AA^{-3}\end{array}$ & $0.58 /-0.46$ & $1.38 /-1.12$ & $1.85 /-0.99$ & $0.97 /-0.74$ \\
\hline
\end{tabular}




\section{Reference}

(1) Block, E.; Ofori-Okai, G.; Zubieta, J. 2-Phosphino- and 2-Phosphinylbenzenethiols: New Ligand Types. J. Am. Chem. Soc. 1989, 111, 2327-2329.

(2) Asdar, A.; Tudoret, M.-J.; Lapinte, C. Cationic (Pentamethylcyclopentadienyl)molybdenum Complexes: Improved Synthesis and Characterization. J. Organomet. Chem. 1988, 349, 353-366.

(3) Shaw, W. J.; Linehan, J. C.; Szymczak, N. K.; Heldebrant, D. J.; Yonker, C.; Camaioni, D. M.; Baker, R. T.; Autrey, T. In Situ Multinuclear NMR Spectroscopic Studies of the Thermal Decomposition of Ammonia Borane in Solution. Angew. Chem. Int. Ed. 2008, 47, 7493-7496.

(4) Galasso, V.; Fronzoni, G. Ab initio Study of the $J(\mathrm{BB})$ and $J(\mathrm{BH})$ Coupling Constants in Polyboranes via the Coupled-Hartree-Fock and Equations-of-Motion Methods. J. Chem. Phys.1986, 85, 5200-5203.

(5) Erman, M. B.; Snow, J. W.; Williams, M. J. A New Efficient Method for the Conversion of Aldehydes into Nitriles Using Ammonia and Hydrogen Peroxide. Tetrahedron Lett. 2000, 41, 6749-6752.

(6) Ramachandran, P. V.; Gagare, P. D. Preparation of Ammonia Borane in High Yield and Purity, Methanolysis, and Regeneration. Inorg. Chem. 2007, 46, 7810-7817.

(7) Ansyln, E. V. Dougherty, D. A. Modern Physical Organic Chemistry. University Science books, 2006.

(8) Espenson, J. H. Chemical Kinetics and Reaction Mechanisms. (2ndedn) McGrawHill, Inc, 2002.

(9) Pilling, M. J. Seakins, P. W. Reaction Kinetics. Oxford University Press, 1995.

(10) Meek, S. J.; Pitman, C. L.; Miller, A. J. M., Deducing Reaction Mechanism: A Guide for Students, Researchers, and Instructors. J. Chem. Educ. 2016, 93, 275-286.

(11)Gaussian 16, R. A., M. J. Frisch, G. W. Trucks, H. B. Schlegel, G. E. Scuseria, M. A. Robb, J. R. Cheeseman, G. Scalmani, V. Barone, G. A. Petersson, H. Nakatsuji, X. Li, M. Caricato, A. V. Marenich, J. Bloino, B. G. Janesko, R. Gomperts, B. Mennucci, H. P. Hratchian, J. V. Ortiz, A. F. Izmaylov, J. L. Sonnenberg, D. Williams-Young, F. Ding, F. Lipparini, F. Egidi, J. Goings, B. Peng, A. Petrone, T. Henderson, D. Ranasinghe, V. G. 
Zakrzewski, J. Gao, N. Rega, G. Zheng, W. Liang, M. Hada, M. Ehara, K. Toyota, R. Fukuda, J. Hasegawa, M. Ishida, T. Nakajima, Y. Honda, O. Kitao, H. Nakai, T. Vreven, K. Throssell, J. A. Montgomery, Jr., J. E. Peralta, F. Ogliaro, M. J. Bearpark, J. J. Heyd, E. N. Brothers, K. N. Kudin, V. N. Staroverov, T. A. Keith, R. Kobayashi, J. Normand, K. Raghavachari, A. P. Rendell, J. C. Burant, S. S. Iyengar, J. Tomasi, M. Cossi, J. M. Millam, M. Klene, C. Adamo, R. Cammi, J. W. Ochterski, R. L. Martin, K. Morokuma, O. Farkas, J. B. Foresman, and D. J. Fox, Gaussian, Inc., Wallingford CT, 2016., Gaussian 16, Revision A.03, M. J. Frisch, G. W. Trucks, H. B. Schlegel, G. E. Scuseria, M. A. Robb, J. R. Cheeseman, G. Scalmani, V. Barone, G. A. Petersson, H. Nakatsuji, X. Li, M. Caricato, A. V. Marenich, J. Bloino, B. G. Janesko, R. Gomperts, B. Mennucci, H. P. Hratchian, J. V. Ortiz, A. F. Izmaylov, J. L. Sonnenberg, D. Williams-Young, F. Ding, F. Lipparini, F. Egidi, J. Goings, B. Peng, A. Petrone, T. Henderson, D. Ranasinghe, V. G. Zakrzewski, J. Gao, N. Rega, G. Zheng, W. Liang, M. Hada, M. Ehara, K. Toyota, R. Fukuda, J. Hasegawa, M. Ishida, T. Nakajima, Y. Honda, O. Kitao, H. Nakai, T. Vreven, K. Throssell, J. A. Montgomery, Jr., J. E. Peralta, F. Ogliaro, M. J. Bearpark, J. J. Heyd, E. N. Brothers, K. N. Kudin, V. N. Staroverov, T. A. Keith, R. Kobayashi, J. Normand, K. Raghavachari, A. P. Rendell, J. C. Burant, S. S. Iyengar, J. Tomasi, M. Cossi, J. M. Millam, M. Klene, C. Adamo, R. Cammi, J. W. Ochterski, R. L. Martin, K. Morokuma, O. Farkas, J. B. Foresman, and D. J. Fox, Gaussian, Inc., Wallingford CT, 2016.

(12) (a) Zhao, Y.; Truhlar, D. G. A New Local Density Functional for Main Group Thermochemistry, Transition Metal Bonding, Thermochemical Kinetics, and Noncovalent Interaction. J. Chem. Phys. 2006, 125, 194101. (b) Zhao, Y.; Truhlar, D. G. Density Functionals with Broad Applicability in Chemistry. Acc. Chem. Res. 2008, 41, 157-167.

(13) Weigend, F.; Ahlrichs, R. Balanced Basis Sets of Split Valence, Triple Zeta Valence and Quadruple Zeta Valence Quality for H to Rn: Design and Assessment of Accuracy. Phy. Chem. Chem. Phy. 2005, 7, 3297-3305.

(14) Marenich, A. V.; Cramer, C. J.; Truhlar, D. G. Universal Solvation Model Based on Solute Electron Density and on a Continuum Model of the Solvent Defined by the Bulk Dielectric Constant and Atomic Surface Tensions. J. Phys. Chem. B 2009, 113, 6378-6396.

(15) Zhao, Y.; Truhlar, D. G. The M06 Suite of Density Functionals for Main Group Thermochemistry, Thermochemical Kinetics, Noncovalent Interactions, Excited States, and Transition Elements: Two New Functionals and Systematic Testing of Four M06Class Functionals and 12 Other Function. Theor. Chem. Acc. 2008, 120 (1-3), 215-241.

(16) (a) A. V. Marenich.; J. Ho, M. Coote.; C. J. Cramer.; D. G. Truhlar. Computational 
Electrochemistry: Prediction of Liquid-Phase Reduction Potentials. Phys. Chem. Chem. Phys. 2014, 16, 15068-15106. (b) Morello, G. R.; Hopmann, K. H. A Dihydride Mechanism Can Explain the Intriguing Substrate Selectivity of Iron-PNP-Mediated Hydrogenation. ACS Catal. 2017, 7, 5847-5855.

(17) Ibrahim, A. D.; Entsminger, S. W.; Fout, A. R. Insights into a Chemoselective Cobalt Catalyst for the Hydroboration of Alkenes and Nitriles. ACS Catal. 2017, 7, 37303734.

(18) Dai, H.; Guan, H. Switching the Selectivity of Cobalt-Catalyzed Hydrogenation of Nitriles. ACS Catal. 2018, 8, 9125-9130.

(19) Cal, P. M. S. D.; Vicente, J. B.; Pires, E.; Coelho, A. V.; Veiros, L. s. F.; Cordeiro, C.; Gois, P. M. P. Iminoboronates: A New Strategy for Reversible Protein Modification. J. Am. Chem. Soc. 2012, 134, 10299-10305.

(20) Bornschein, C.; Werkmeister, S.; Wendt, B.; Jiao, H.; Alberico, E.; Baumann, W.; Junge, H.; Junge, K.; Beller, M. Mild and Selective Hydrogenation of Aromatic and Aliphatic (di)nitriles with a Well-Defined Iron Pincer Complex. Nat. Commun. 2014, 5, 4111-4121. 